\title{
Design of a Tip Appendage for the Control of Tip Leakage Vortices in Axial Flow Fans
}

\author{
by \\ Thomas Oliver Meyer \\ Dissertation presented for the degree of Doctor of Philosophy \\ (Mechanical Engineering) in the Faculty of Engineering at \\ Stellenbosch University
}

Supervisor: Prof. Sybrand Johannes van der Spuy Co-supervisors: Prof. Christiaan Johannes Meyer Prof. Alessandro Corsini

March 2021 


\section{Declaration}

By submitting this dissertation electronically, I declare that the entirety of the work contained therein is my own, original work, that I am the sole author thereof (save to the extent explicitly otherwise stated), that reproduction and publication thereof by Stellenbosch University will not infringe any third party rights and that I have not previously in its entirety or in part submitted it for obtaining any qualification.

Date: $01-10-2020$

Copyright (C) 2021 Stellenbosch University All rights reserved. 


\title{
Abstract
}

\author{
Department of Mechanical and Mechatronic Engineering, \\ University of Stellenbosch, \\ Private Bag X1, 7602 Matieland, South Africa. \\ Dissertation: PhD Eng (Mech)
}

2021

The design of a tip appendage for the passive control of tip leakage vortices in axial flow fans is investigated. A periodic three-dimensional numerical model of a $1.542 \mathrm{~m}$ (casing) diameter subsonic axial flow fan, designed for use in air-cooled heat exchangers (ACHEs), is developed using the open-source CFD libary OpenFOAM (OF) v1806. The implemented meshing strategy, which uses a combination of the cross-platform libraries cfMesh and SnappyHexMesh (SHM), is found to sufficiently validate against experimental data with a root-mean-square error $(R M S E)$ of $3.8 \mathrm{~Pa}$ in total-to-static pressure rise, $129.6 \mathrm{~W}$ in fan shaft power consumption, and 1.5 percentage points in total-to-static efficiency for the datum fan case.

Steady and unsteady numerical computations are used to determine the effect of tip clearance on fan performance characteristics. An increase of 15.6 percent in total-to-static pressure rise and a 5.1 percentage point increase in total-to-static efficiency is found with a two-thirds reduction in tip clearance at the fan's design point flow rate. Normalized helicity contours are used in the visualization and quantification of the flow near the blade tip. The findings show a reduction in the relative tip leakage flow (TLF) velocity, $W_{T L F}$, and tip leakage vortex (TLV) exit trajectory angle, $\beta_{T L V}$, along the blade chord with a reduction in tip clearance.

These two flow characteristics are numerically investigated with the aim of controlling them through implementation of two tip appendage designs, these being a constant thickness (CT) end-plate and novel trailing edge (TE) end-plate design. A numerical comparison between the two designs indicates that the new TE 
end-plate concept improves the fan performance characteristics through exhibiting control of the relative TLV exit trajectory angle. Consequently, this design is chosen with the aim of further improving the fan aerodynamic performance characteristics through optimization.

The Design and Analysis of Computer aided Experiments (DACE) method is used in the construction of the Kriging based surrogate model's database. The resulting database is coupled with an Efficient Global Optimization (EGO) algorithm which completes the workflow of the multi-objective multi-point (MOMP) optimization process. The Pareto-front of non-dominated solutions is used to guide the optimal design selection, on which the experimental evaluations are based. The results of the optimized design indicate improved fan performance characteristics at greater than peak efficiency flow rates. This design is found to increase the datum fan's design point performance characteristics by a value of 32.90 percent in total-tostatic pressure rise and a 7.66 percentage point increase in total-to-static efficiency at the fan's design speed of $722 \mathrm{rpm}$. 


\title{
Uittreksel
}

\author{
Departement Meganiese en Megatroniese Ingenieurswese, \\ Universiteit van Stellenbosch, \\ Privaatsak X1, 7602 Matieland, Suid Afrika. \\ Proefskrif: PhD Ing (Meg)
}

2021

Die ontwerp van 'n puntaanhangsel vir die passiewe beheer van puntlekwerwels in aksiaalvloeiwaaiers is ondersoek. 'n Periodieke drie-dimensionele numeriese model van 'n subsoniese aksiaalvloeiwaaier, vir gebruik in lugverkoeldewarmteruilers, is met behulp van die oopbronprogrammatuur OpenFOAM (OF) v1806 ontwerp. Daar is bevind dat die nuut-geïmplementeerde roosterstrategie, wat 'n kombinasie van die kruis-platformbiblioteke cfMesh en SnappyHexMesh (SHM) gebruik, voldoende gevalideer is met ' $\mathrm{n}$ wortel-gemiddelde-kwadraat afwyking van $3.8 \mathrm{~Pa}$ in totale-tot-statiese-druk, $129.6 \mathrm{~W}$ in waaierdrywingsverbruik en 1.5 persent in totale-tot-statiese-benuttingsgraad.

Gestadigde en ongestadigde numeriese berekeninge is gebruik om die effek van puntspeling op waaierprestasiekarakteristieke te bepaal. 'n Toename van 15.6 persent in totale-tot-statiese-druk en 'n 5.1 persent toename in totale-tot-statiesebenuttingsgraad is met ' $\mathrm{n} 66.7$ persent afname in puntspeling gevind by die waaier se ontwerp operasionele vloeitempo. Daar is van genormaliseerde helisiteitskontoere gebruik gemaak om die vloei digby die lempunt te visualiseer en te kwantifiseer. Die bevindinge dui op 'n vermindering in die puntlekvloei en die uitgangsbaanhoek, $\beta_{P L W}$, van die puntlekwerwel langs die lemkoord af met 'n afname in puntvryruimte.

Hierdie twee vloeikenmerke word numeries ondersoek met die doel om dit deur die inwerkingstelling van twee puntaanhangselontwerpe te beheer, naamlik 'n eindplaatontwerp met konstante dikte (KD) en 'n nuwe eindplaatontwerp met 'n agterrand (AR). 'n Numeriese vergelyking tussen die twee ontwerpe dui aan dat die 
nuwe AR-eindplaatkonsep die waaier se prestasiekenmerke verbeter deur beheer oor die hoek van die relatiewe puntlekkasiedraaikolk se uitgangstrajek uit te oefen. Gevolglik word hierdie ontwerp gekies ten einde die waaier se aërodinamiese prestasiekenmerke verder deur optimering te verbeter.

Die metode vir die ontwerp en ontleding van eksperimente met rekenaarsteun [Design and Analysis of Computer-aided Experiments (DACE)] is in die konstruksie van die Kriging-gebaseerde surrogaatmodel se databasis gebruik. Die gevolglike databasis word aan 'n algoritme vir doeltreffende globale optimalisering gekoppel, wat die werksvloei van die multidoelwit multipunt optimerings voltooi. Die Pareto-front van nie-gedomineerde oplossings word gebruik om die optimale ontwerpkeuse waarop die eksperimentele evaluerings gegrond word, te rig. Die resultate van die geoptimeerde ontwerp dui op verbeterde waaierprestasiekenmerke teen vloeitempo's groter as piek benuttingsgraad. Hierdie ontwerp verbeter die verwysingswaaier se ontwerppunt-prestasiekenmerke met 'n verhoging van 32.90 persent in totaal-tot-statiese druk en 'n verhoging van 7.66 persentasiepunte in totaal-tot-statiese benuttingsgraad teen die waaier se ontwerpspoed van $722 \mathrm{rpm}$. 


\section{Acknowledgements}

First and foremost, I would like to sincerely thank my family for their endless love and support, without which I would not be where I am today. To my mom and dad, I cannot begin to show you the amount of gratitude I have for what you have done for me and a simple thank you is not enough to show my appreciation.

To my supervisors here in South Africa, Prof. van der Spuy and Prof. Meyer, thank you for your tireless efforts and guidance throughout this (now) dissertation! I hope to have many more beer/coffee sessions in the future. Prof. Meyer, as wacky as I think you are and as much as I hate to admit it; it 'seems' that it has only been average working with you. Prof. van der Spuy, I know that I have said it before but I will say it again, thank you so much for organizing and giving me so many opportunities to grow.

To my supervisors in Italy, Prof. Corsini, and Prof. Delibra, 'grazie mille' for all your help in furthering my knowledge and understanding in not only the world of computational fluid dynamics. Alessandro, when you visit again I hope that we can go for that beer. To all the colleagues and friends that I have had the privilege of working with and meeting while in Italy, thank you for making me feel so welcomed.

To all my friends and colleagues, thank you for all of your contributions and help throughout the duration of this thesis. A special thank you to Thomas Ochabski and Zander Meiring. Your support and friendship has been invaluable to me.

A special thank you to David Volponi. David, I appreciate the effort you put in to make me feel so welcomed in Italy.

All computations were performed using the facilities provided by the Centre for High Performance Computing.

Last but not least the funders. Thank you to the MinWaterCSP project for provid- 
ing the necessary funding for the exchange to Italy, the Hillensberg Trust bursary for providing financial support during my postgraduate studies and Stellenbosch University for providing me with a departmental bursary and the facilities to complete my work. 
To my family and friends 


\section{Contents}

Declaration i

Abstract ii

Uittreksel iv

Contents $\quad$ ix

List of Figures xiv

List of Tables $\quad$ xix

$\begin{array}{lll}1 & \text { Introduction } & 1\end{array}$

1.1 Background $\ldots . \ldots \ldots \ldots \ldots$

1.2 Test fan . . . . . . . . . . . . . . . . . . . . . 3

1.3 Research objectives and methodology . . . . . . . . . . . . . . . . 4

\begin{tabular}{lll}
\hline 2 & Literature review & 7
\end{tabular}

2.1 Tip leakage flow . . . . . . . . . . . . . . . . . . . . . . . . . . . . . . 7

2.2 Tip effects on fan performance . . . . . . . . . . . . . . . . . . . 8

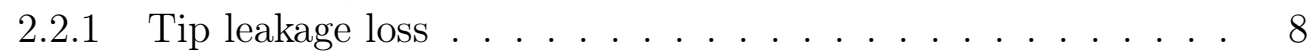

$2.2 .2 \quad$ Tip clearance $\ldots \ldots \ldots \ldots \ldots$

2.2 .3 Tip geometry $\ldots \ldots \ldots \ldots$

2.2 .4 Boundary layer effects within the tip region . . . . . . . . . 12

2.3 Tip modification techniques . . . . . . . . . . . . . . . . . 12

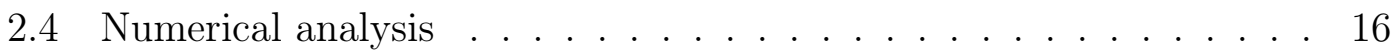

2.4 .1 Three-dimensional modelling of an axial flow fan. . . . . . . 16

2.4 .2 Open-source meshing . . . . . . . . . . . . . . . 17

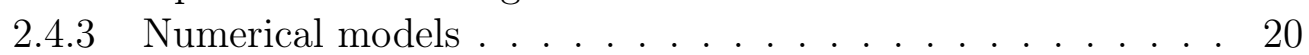

2.5 Optimization techniques . . . . . . . . . . . . . . . . . 21

2.5 .1 Surrogate models . . . . . . . . . . . . . . . . . 22

2.5 .2 Search algorithms . . . . . . . . . . . . . . . . 22

ix 
2.6 Optimization objective function . . . . . . . . . . . . . . . . 26

2.6 .1 Single-objective optimization. . . . . . . . . . . . . . . 27

2.6 .2 Multi-objective optimization . . . . . . . . . . . . . . . 27

2.7 Optimization with constraints . . . . . . . . . . . . . . . . . . 29

2.7 .1 Boundary constraints . . . . . . . . . . . . . . . . 30

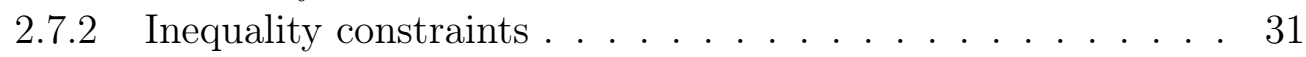

2.8 Concluding remarks $\ldots \ldots \ldots$. . . . . . . . . . . . . . . . . 33

$\begin{array}{llr}3 & \text { Periodic three-dimensional numerical model } & 36\end{array}$

3.1 Computational domain . . . . . . . . . . . . . . . . 36

3.2 Meshing . . . . . . . . . . . . . . . . . . . . 37

3.2 .1 Strategy . . . . . . . . . . . . . . . . . . . . . . . . . . . . . . . . . . .

3.2 .2 Construction . . . . . . . . . . . . . . . . . . . . 41

3.3 Boundary conditions . . . . . . . . . . . . . . . . . . . . . . . . . . . . . . . . . . . . 42

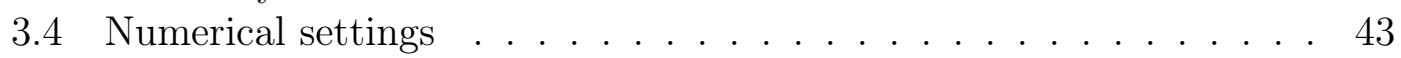

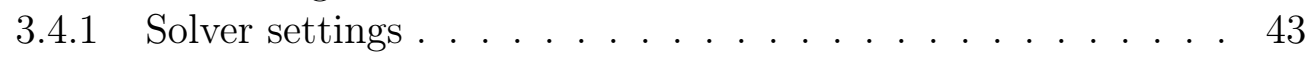

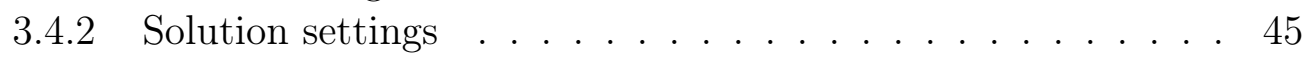

3.4 .3 Solution initialization and convergence . . . . . . . . . . . . . 45

3.5 Turbulence modelling . . . . . . . . . . . . . . . . . . 47

\begin{tabular}{|lll}
\hline & Periodic three-dimensional numerical model validation & 49
\end{tabular}

4.1 Definition of fan performance characteristics . . . . . . . . . . . . . 49

4.2 Aerodynamic performance comparison . . . . . . . . . . . . . . 50

4.3 Validation of results $\ldots \ldots \ldots \ldots \ldots$. . . . . . . . . . . . 52

4.3 .1 Variation . . . . . . . . . . . . . . . . . 52

$4.3 .2 \operatorname{Bias} \ldots \ldots \ldots \ldots \ldots \ldots \ldots$

5 Effect of tip clearance and tip leakage vortex development $\quad 55$

5.1 Fan scaling at various tip clearances . . . . . . . . . . . . . 55

5.2 Aerodynamic performance analysis $\ldots \ldots \ldots$. . . . . . . . 57

5.3 Vortex detection and visualization . . . . . . . . . . . . . . . . . . . . 58

5.4 Vortex quantification and analysis . . . . . . . . . . . . . . . . . 60

5.5 Blade surface pressure distribution . . . . . . . . . . . . . . . . . 62

5.6 Chapter summary . . . . . . . . . . . . . . . . . . 63

$\begin{array}{llr}6 & \text { Tip appendage design } & 65\end{array}$

6.1 Design considerations . . . . . . . . . . . . . . . . . . . . . . . . 65

6.2 Tip leakage flow control . . . . . . . . . . . . . . . . . . 65

6.2 .1 Design . . . . . . . . . . . . . . . . . . 65 65

$6.2 .2 \quad$ Aerodynamic performance analysis . . . . . . . . . . . . 67

6.2 .3 Vortex analysis . . . . . . . . . . . . . . 70 
$6.2 .4 \quad$ Blade surface pressure distribution . . . . . . . . . . . 73

6.3 Tip leakage vortex exit trajectory angle control . . . . . . . . . . . 74

$6.3 .1 \quad$ Design . . . . . . . . . . . . . . . . . . 74

6.3 .2 Aerodynamic performance analysis . . . . . . . . . . 77

6.3 .3 Vortex analysis . . . . . . . . . . . . . . . . . . . 79

6.3 .4 Blade surface pressure distribution . . . . . . . . . . . . . . . 81

6.4 Chapter summary . . . . . . . . . . . . . . 82

$\begin{array}{llr}7 & \text { Design screening } & 84\end{array}$

7.1 Design selection . . . . . . . . . . . . . . . . . . 84

7.2 Optimization problem definition . . . . . . . . . . . . . . . . 85

7.2 .1 Objective function $\ldots \ldots \ldots \ldots$. . . . . . . . . . . . . . . . . . . . 85

7.2 .2 Boundary constraints . . . . . . . . . . . . . . . . . 86

$7.2 .3 \quad$ Inequality constraints . . . . . . . . . . . . . . . . . . . . 89

7.3 Chapter summary . . . . . . . . . . . . . . . . . . . 90

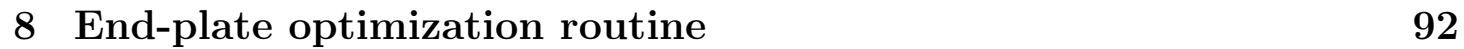

8.1 Background . . . . . . . . . . . . . . . . . . . 92

8.2 Design space population . . . . . . . . . . . . . . . . . . . . . . . . . . . . . . . . . . . . . . 93

8.2 .1 Sampling method . . . . . . . . . . . . . . . . . . . . 93

8.2 .2 Database construction . . . . . . . . . . . . . . . . . . . . 95

8.3 The DACE surrogate model . . . . . . . . . . . . . . . . . . . . . . 97

8.3 .1 Overview . . . . . . . . . . . . . . . . . . . 97

8.3 .2 Error correlation . . . . . . . . . . . . . . . . . . . . 98

8.3 .3 Parameter estimation . . . . . . . . . . . . . . . . . . . 100

8.3 .4 The DACE predictor . . . . . . . . . . . . . . . . . . . . 104

8.4 Model error and validation . . . . . . . . . . . . . . . . . . 105

8.4 .1 Model error . . . . . . . . . . . . . . . . . . . 105

8.4 .2 Cross-validation . . . . . . . . . . . . . . . . 105

8.5 Implementing the DACE model . . . . . . . . . . . . . . . . . . . 108

$8.5 .1 \quad$ Optimal hyperparameter estimation . . . . . . . . . . . . . . 109

8.5 .2 Hyperparameter initialization and bounds . . . . . . . . . . 111

8.5 .3 Regularization . . . . . . . . . . . . . . . . . . . 114

8.6 The EGO algorithm . . . . . . . . . . . . . . . . . . . . . . 117

$8.6 .1 \quad$ Expected improvement . . . . . . . . . . . . . . . . . . 119

8.6.2 Maximum expected improvement estimation . . . . . . . . . 122

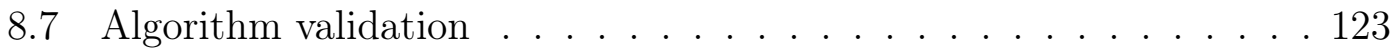

8.7 .1 Definition of performance metrics . . . . . . . . . . . . 123

8.7 .2 Test functions . . . . . . . . . . . . . . . . . . . . . . . . . . . 124

8.7 .3 Results . . . . . . . . . . . . . . . . . . . . . . . . . . 125

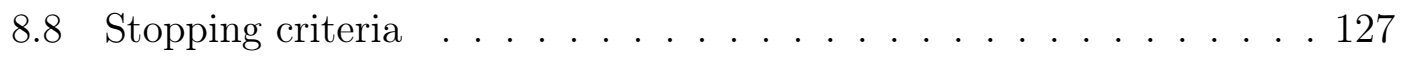




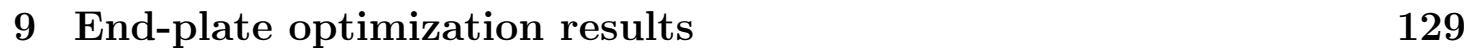

9.1 Optimizer cross-validation . . . . . . . . . . . . . . . . . . . . . . . 129

9.2 Convergence history $\ldots \ldots \ldots$. . . . . . . . . . . . . 130

9.3 Pareto-front . . . . . . . . . . . . . . . . . . . . . . . 133

9.4 Final selected designs . . . . . . . . . . . . . . . . . . . . . . . . 134

9.5 Chapter summary . . . . . . . . . . . . . . . . . . . . . . . 135

10 Experimental method 136

10.1 Design construction . . . . . . . . . . . . . . . . . . . . . 136

10.2 Fan performance testing . . . . . . . . . . . . . . 137

10.2 .1 Test facility . . . . . . . . . . . . . . . . . . . 137

10.2 .2 Facility instrumentation . . . . . . . . . . . . . . . 139

10.2 .3 Testing procedure . . . . . . . . . . . . . . . . . . 141

10.3 Results . . . . . . . . . . . . . . . . . . . . . . . 142

10.3 .1 Aerodynamic performance analysis . . . . . . . . . . . . . . 142

10.3 .2 Operating point comparison . . . . . . . . . . . . . . . . 144

10.3 .3 Angle of attack analysis . . . . . . . . . . . . . . . . 146

10.3 .4 Verification of results . . . . . . . . . . . . . . . . . . 148

11 Conclusion 151

11.1 Objectives and methodology . . . . . . . . . . . . . . . . 151

11.2 Key findings . . . . . . . . . . . . . . . . . . . . . . . . . . . . . . . . . . . . . . . . . . . . . . . . . . . . . . .

11.3 Recommended future work . . . . . . . . . . . . . . . . . . . 155

\begin{tabular}{ll}
\hline References & 156
\end{tabular}

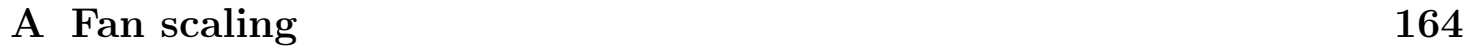

B Periodic three-dimensional numerical model sensitivity analysis 166

B.1 Mesh density sensitivity . . . . . . . . . . . . . . 166

B.2 Inlet/Outlet patch displacement sensitivity . . . . . . . . . . . . . . 169

B.3 Boundary layer thickness effects within the tip region . . . . . . . . 170

B.4 Tip clearance mesh density sensitivity . . . . . . . . . . . . . . . . . 171

\begin{tabular}{ll} 
C & Turbulence properties \\
\hline
\end{tabular}

C.1 Estimation of turbulent kinetic energy from turbulence intensity . . 172

C.2 Estimation of turbulent dissipation/specific dissipation rate from mixing length scale . . . . . . . . . . . . . . . . 172

\begin{tabular}{lr}
\hline D Post-processing & 174
\end{tabular}

D.1 Tip leakage flow quantification . . . . . . . . . . . . . . . . . . . . . 174

D.2 Tip leakage vortex quantification . . . . . . . . . . . . . . . . . . . 175 


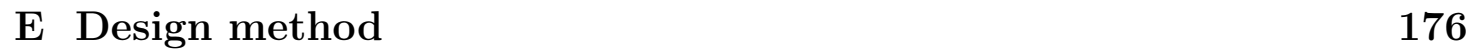

\begin{tabular}{lll}
\hline F & Structural feasibility analysis & 182
\end{tabular}

F.1 Displacement analysis . . . . . . . . . . . . . . . . . . . . . . . . . 182

F.2 $\quad$ Stress analysis . . . . . . . . . . . . . . . . . . . . . . . 186

$\begin{array}{ll}\text { G Test facility calibration } & 188\end{array}$

G.1 Pressure transducer calibration . . . . . . . . . . . . . . . . . . . . 188

G.2 Torque transducer calibration . . . . . . . . . . . . . . . . . . . . 189

G.3 $\quad$ Fan rotational speed calibration . . . . . . . . . . . . . . . . . . . 191

\begin{tabular}{ll}
\hline H Fan characteristics sample calculation & 194
\end{tabular} 


\section{List of Figures}

$1.1 \quad$ Scale M-fan experimental assembly (Wilkinson et al. $[2018)]$. . . . . . 4

1.2 Work flow breakdown $\ldots \ldots \ldots \ldots$. . . . . . . . . . . 6

2.1 Tip leakage flow visualization (Inoue and Kuroumaru, 1989)] . . . . . . 8

2.2 Tip leakage flow visualization with variable blade thicknesses (Denton,

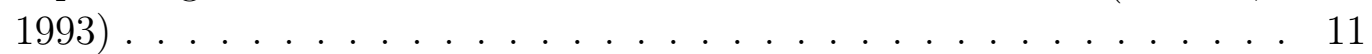

2.3 a) Various tip end-plate designs with b) their respective chord-wise vortex Rossby number (Ro) evolutions at the fan's $7 \mathrm{~m}^{3} / \mathrm{s}$ design operating point (adapted from Corsini et al., 2010) $\ldots$. . . . . . . . . . . . 13

2.4 a) TFmvb end-plate design with b) chord-wise vortex Rossby number evolution at the fan's $7 \mathrm{~m}^{3} / \mathrm{s}$ design operating point (adapted from Corsini and Sheard, 2013) $\ldots \ldots \ldots \ldots \ldots \ldots$

2.5 a) Experimental fan total pressure and b) total efficiency performance curves (adapted from Corsini and Sheard, 2013) $\ldots . . . . . . .1616$

$2.6 \quad$ Detail of cfMesh mesh generation process (Juretić, 2015)] . . . . . . . . . 18

2.7 SnappyHexMesh meshing procedure: a) initial mesh creation, b) castellation, c) snapping, and d) layer addition (OpenCFD Ltd[ 2018)] . . . . 19

2.8 Illustration of the steps of the differential evolution algorithm . . . . . 26

2.9 Multi-objective optimization (adapted from Sudhoff $[2007)$ ) . . . . . . 28

2.10 Constrained vs unconstrained optimization problem . . . . . . . . . . . 29

2.11 Interior and exterior penalty constraint handling . . . . . . . . . . . . . 32

2.12 Direct constraint handling . . . . . . . . . . . . . . . . . . 33

3.1 Periodic three-dimensional numerical model computational domain . . 37

3.2 BaseMesh creation (section A-A Figure 3.1 ) . . . . . . . . . . . . . . . 38

$3.3 \quad$ BaseMesh preparation (section A-A Figure 3.1) . . . . . . . . . . . . . . . 39

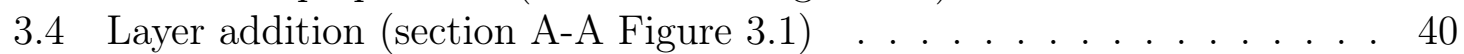

3.5 Periodic three-dimensional numerical model computational mesh . . . . 41

3.6 Solution convergence . . . . . . . . . . . . . . . . 46

\begin{tabular}{|ll}
\hline 4.1 & Scale M-fan experimental and numerical total-to-static pressure rise \\
\hline \hline & comparison at various tip clearances $\ldots \ldots \ldots \ldots$ \\
\hline
\end{tabular} 
4.2 Scale M-fan experimental and numerical power comparison at various

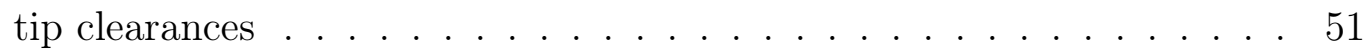

$4.3 \quad$ Scale M-fan experimental and numerical total-to-static efficiency comparison at various tip clearances . . . . . . . . . . . . . . 52

5.1 Normalized helicity $H_{\text {norm }}$ cross sectional contours of the scale M-fan at blade tip with suction side vortex (TLV/SS) visualization at various tip clearances evaluated at their respective design point flow rates: a) $2 \mathrm{~mm}$ tip clearance $\left[V=14.80 \mathrm{~m}^{3} / \mathrm{s}\right]$, b) $4 \mathrm{~mm}$ tip clearance $[V=$ $\left.14.68 \mathrm{~m}^{3} / \mathrm{s}\right]$, and c) $6 \mathrm{~mm}$ tip clearance $\left[V=14.57 \mathrm{~m}^{3} / \mathrm{s}\right] \ldots \ldots 59$

5.2 Blade-to-blade tip leakage vortex interaction of the scale M-fan with a $6 \mathrm{~mm}$ tip clearance at the fan's $14.57 \mathrm{~m}^{3} / \mathrm{s}$ design point flow rate $\quad$. . . 60

$5.3 \quad$ Relative tip leakage flow velocity $\left(W_{T L F}\right)$ and suction side vortex exit trajectory angle $\left(T L V / S S, \beta_{T L V}\right)$ of the scale M-fan at various tip clearances evaluated at their respective design point flow rates: $2 \mathrm{~mm}$ tip clearance $\left[\dot{V}=14.80 \mathrm{~m}^{3} / \mathrm{s}\right], 4 \mathrm{~mm}$ tip clearance $\left[V=14.68 \mathrm{~m}^{3} / \mathrm{s}\right]$, and $6 \mathrm{~mm}$ tip clearance $\left[V=14.57 \mathrm{~m}^{3} / \mathrm{s}\right][\ldots \ldots . \ldots 61$

5.4 Chord-wise static pressure coefficient distributions $C p$ of the scale Mfan at various tip clearances evaluated at $91.6 \%$ blade span at the fan's design point flow rate. . . . . . . . . . . . . . 63

6.1 Constant thickness (CT) end-plate designs: a) datum, b) $24 \mathrm{~mm}$ pressure side end-plate, c) $47 \mathrm{~mm}$ pressure side end-plate, and d) $24 \mathrm{~mm}$ suction side end-plate. . . . . . . . . . . . . . . . 67

6.2 Constant thickness end-plate designs and datum fan numerical totalto-static pressure rise comparison . . . . . . . . . . . . 68

6.3 Constant thickness end-plate designs and datum fan numerical power

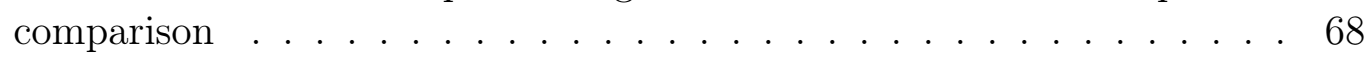

6.4 Constant thickness end-plate designs and datum fan numerical totalto-static efficiency comparison . . . . . . . . . . . . . . . 69

6.5 Normalized helicity $H_{\text {norm }}$ cross sectional contours of constant thickness end-plate designs at blade tip with suction side vortex (TLV/SS) visualization evaluated at the fan's $14.57 \mathrm{~m}^{3} / \mathrm{s}$ design point flow rate for a $6 \mathrm{~mm}$ tip clearance: a) $24 \mathrm{~mm}$ pressure side end-plate, b) $47 \mathrm{~mm}$ pressure side end-plate and c) $24 \mathrm{~mm}$ suction side end-plate $\quad \ldots . .71$

6.6 Relative tip leakage flow velocity $\left(W_{T L F}\right)$ and suction side vortex exit trajectory angle $\left(T L V / S S, \beta_{T L V}\right)$ of constant thickness end-plate designs evaluated at the fan's $14.57 \mathrm{~m}^{3} / \mathrm{s}$ design point flow rate for a $6 \mathrm{~mm}$ tip clearance . . . . . . . . . . . . . . 72 
6.7 Chord-wise static pressure coefficient distributions $C p$ of constant thickness end-plate designs compared to fan datum evaluated at $91.6 \%$ blade span at the fan's design point flow rate . . . . . . . . . . . . . 73

6.8 Trailing edge (TE) end-plate design . . . . . . . . . . . . . . . . . . 75

6.9 Flat plate lift coefficient and moment coefficient about the mid-chord versus angle of attack (adapted from Amandolese et al., 2013) . . . . . 76

6.10 Trailing edge end-plate designs and datum fan numerical total-to-static pressure rise comparison . . . . . . . . . . . . . . 77

6.11 Trailing edge end-plate designs and datum fan numerical power com-

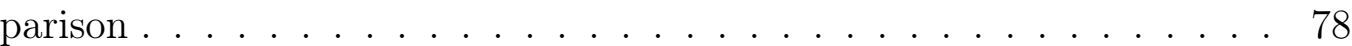

6.12 Trailing edge end-plate designs and datum fan numerical total-to-static efficiency comparison . . . . . . . . . . . . . . . 78

6.13 Normalized helicity $H_{\text {norm }}$ cross sectional contours of trailing edge endplate designs at blade tip with suction side vortex (TLV/SS) visualization evaluated at the fan's $14.57 \mathrm{~m}^{3} / \mathrm{s}$ design point flow rate for a $6 \mathrm{~mm}$ tip clearance: a) $\zeta_{e p}=0^{\circ}$, b) $\zeta_{e p}=4^{\circ}$, and c) $\zeta_{e p}=15^{\circ} \ldots \ldots$. . . . . 80

6.14 Tip leakage flow velocity $\left(W_{T L F}\right)$ and suction side vortex exit trajectory angle $\left(T L V / S S, \beta_{T L V}\right)$ of trailing edge end-plate designs evaluated at the fan's $14.57 \mathrm{~m}^{3} / \mathrm{s}$ design point flow rate for a $6 \mathrm{~mm}$ tip clearance $\quad$. . 81

6.15 Chord-wise static pressure coefficient distributions $C p$ of trailing edge end-plate designs compared to fan datum evaluated at $91.6 \%$ blade span at the fan's design point flow rate . . . . . . . . . . . . . 82

7.1 Two-level, full factorial design $\left(k=3\right.$ and $\left.2^{k}=8\right) \ldots$. . . . . . . . 87

7.2 $\quad$ Two-level, full factorial design of TE end-plate design . . . . . . . . . . . 88

7.3 Graphical illustration with true (solid) and modified (dashed) 1-dimensional response curves of adapted bounce-back resetting scheme for replacing non-feasible response values . . . . . . . . . . . . . . . . 990

8.1 Comparison of two different Latin hypercubes . . . . . . . . . . . . . . 94

8.2 Initial DACE database using LHSMDU method for TE end-plate design 96

8.3 The DACE surrogate model concept - the differences in prediction between (a) an uncorrelated process and (b) a correlated process (adapted from Bergh (2018)). As the prediction at $x^{*}$ is close to that of $x^{i}$ in (b), it can be adjusted to take into account for the positive residual at $x^{i}$. Conversely, there is no correlation in (a). . . . . . . . . . . 98

8.4 Influence of DACE parameters on correlation function . . . . . . . . . 99

8.5 Simple and Ordinary Kriging example . . . . . . . . . . . . . . . . . . 102

8.6 Universal Kriging example . . . . . . . . . . . . . . . . . . . . . . . . . 103

8.7 A well (left) and poorly (right) fit DACE model . . . . . . . . . . . . . 108 
8.8 Effect of random uniform (top) and log-uniform (bottom) hyperparameter space initialization on the concentrated ln-likelihood function of Rosenbrock's 2D valley test function . . . . . . . . . . . . . . . 113

8.9 The EGO algorithm . . . . . . . . . . . . . . . . . . . . . . . . 118

8.10 Simple sampling of the response surface . . . . . . . . . . . . . . . . . 119

8.11 Influence of $g$ parameter on EI function for a simple 1-D function with $f(x)=\sin (x)+\sin (10 x / 3) \ldots \ldots \ldots \ldots 122$

9.1 DACE model validation of the TE end-plate design optimization . . . . 130

9.2 Convergence history of the TE end-plate optimization routine . . . . . . 131

9.3 Spatial convergence history of TE end-plate optimization: Initial database

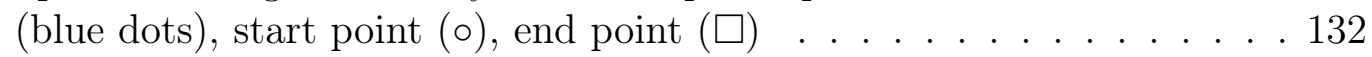

9.4 Pareto-front of multi-objective, multi-point TE end-plate optimization

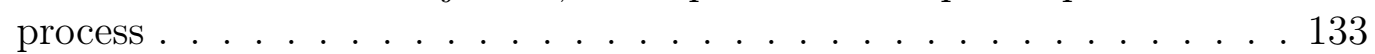

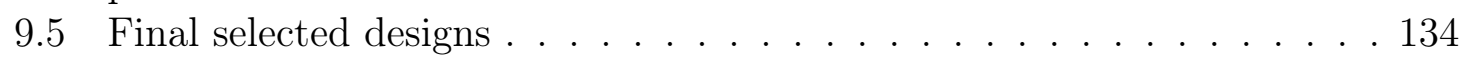

10.1 Final end-plate design . . . . . . . . . . . . . . . . . . . . . 137

10.2 Stellenbosch University ISO 5801, type A test facility (adapted Louw

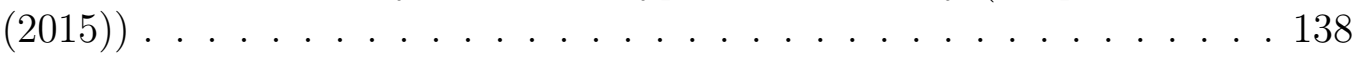

10.3 Test fan set-up . . . . . . . . . . . . . . . . . . . . . . . . . 139

10.4 Test facility instrumentation acquisition system (adapted from Louw,

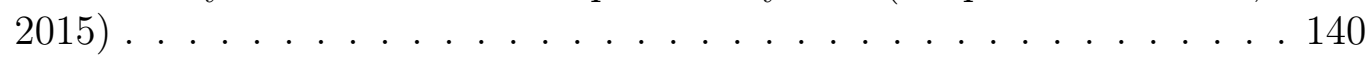

10.5 Experimental and numerical trailing edge end-plate and datum fan total-to-static pressure rise comparison . . . . . . . . . . . . 142

10.6 Experimental and numerical trailing edge end-plate and datum fan power comparison . . . . . . . . . . . . . . . 143

10.7 Experimental and numerical trailing edge end-plate and datum fan total-to-static efficiency comparison . . . . . . . . . . . . . . 143

10.8 Experimental total-to-static pressure rise comparison between increasing blade stagger angle and optimized TE end-plate design . . . . . . . 146

10.9 Experimental power comparison between increasing blade stagger angle

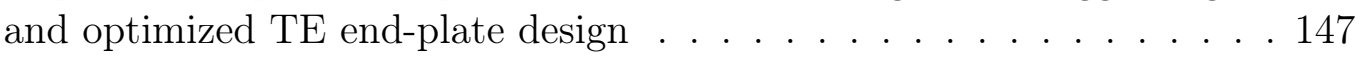

10.10Experimental total-to-static efficiency comparison between increasing blade stagger angle and optimized TE end-plate design . . . . . . . . . 147

B.1 Rotor region mesh refinement . . . . . . . . . . . . . . . . . . . . 167

B.2 Wall boundary allocation . . . . . . . . . . . . . . . . . . . 170

D.1 Area-weighted average relative tip leakage flow velocity post-processing:

a) Paraview filter example and b) Paraview visualization . . . . . . . . 174

D.2 Tip leakage vortex exit trajectory angle post-processing . . . . . . . . . . 175 
E.1 Design construction approach and initial set-up . . . . . . . . . . . . . 178

E.2 $\quad$ Layering method . . . . . . . . . . . . . . . . . . . . . . . . . . . . . 179

E.3 $\quad$ Pre-curing set-up . . . . . . . . . . . . . . . . . . . . . . . 180

E.4 Final end-plate design . . . . . . . . . . . . . . . . . . . . . . . . . . . 181

F.1 Trailing edge end-plate dimensions . . . . . . . . . . . . . . . . . . . . 182

F.2 $\quad$ Fixed-free cantilever beam set-up . . . . . . . . . . . . . . . . . . . . 183

F.3 Midpsan static pressure coefficient $C p$ distribution of the optimized TE end-plate design evaluated at $\dot{V}=9.29 \mathrm{~m}^{3} / \mathrm{s} \ldots \ldots \ldots$. . . . . . 184

F.4 Optimized TE end-plate maximum displacement vs end-plate thickness evaluated at $V=9.29 \mathrm{~m}^{3} / \mathrm{s} \ldots \ldots \ldots \ldots . \ldots . \ldots . \ldots 185$

F.5 $\quad$ Annular disk radial and hoop stress distributions . . . . . . . . . . . . 186

G.1 Bellmouth (left) and plenum chamber (right) pressure transducers . . . 188

G.2 Pressure transducer calibration results . . . . . . . . . . . . . . . . . . 189

G.3 Torque transducer calibration . . . . . . . . . . . . . . . . . . . . . 190

G.4 Torque transducer calibration results . . . . . . . . . . . . . . . . . . . 191

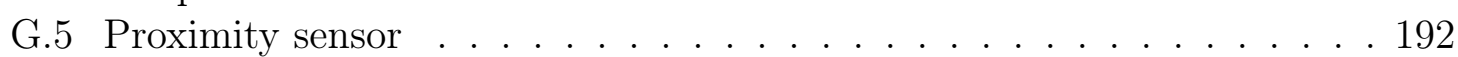

G.6 Proximity sensor calibration results . . . . . . . . . . . . . . . . . 192 


\section{List of Tables}

1.1 M-fan specifications (Wilkinson, $[2017)] \ldots$. . . . . . . . . . . . 3

3.1 Mesh quality control parameters . . . . . . . . . . . . . . . . . . . . 42

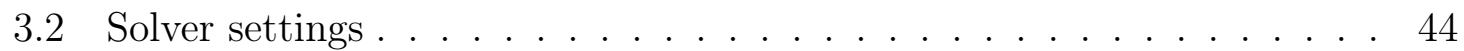

3.3 Solution settings $\quad \ldots \ldots \ldots \ldots \ldots$. . . . . . . . . . . . . . . . . . . . 45

3.4 Turbulence model comparison of the scale M-fan at the $14.68 \mathrm{~m}^{3} / \mathrm{s}$ calculated design flow rate of Wilkinson et al. $[(2019)$ for a $6 \mathrm{~mm}$ tip clearance. . . . . . . . . . . . . . . . . 4 47

4.1 Comparison of numerical root-mean-square error and maximum experimental test facility standard deviation of the scale M-fan at various

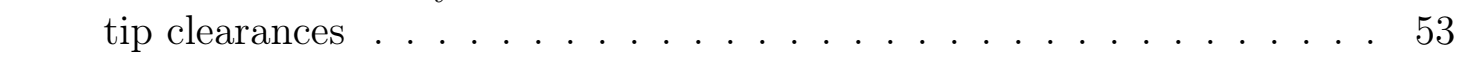

4.2 Difference in numerical and experimental results of the scale M-fan compared to literature evaluated at each fan's respective design point flow rate . . . . . . . . . . . . . . . . . 54

5.1 Scale M-fan specifications for various tip clearances $\left[N_{m}=722 \mathrm{rpm}\right]$. . 56

5.2 Effect of scaling design point flow rate on scale M-fan experimental results (Wilkinson et al. [2019)] . . . . . . . . . . . . . . . . 56

$5.3 \quad$ Numerical fan performance characteristics of the scale M-fan at various tip clearances evaluated at their respective design point flow rates . . . 58

6.1 Numerical fan performance characteristics of constant thickness endplate designs compared to fan datum evaluated at the fan's $14.57 \mathrm{~m}^{3} / \mathrm{s}$ design point flow rate for a $6 \mathrm{~mm}$ tip clearance . . . . . . . . . . . 70

6.2 Numerical fan performance characteristics of trailing edge end-plate designs compared to fan datum evaluated at the fan's $14.57 \mathrm{~m}^{3} / \mathrm{s}$ design point flow rate for a $6 \mathrm{~mm}$ tip clearance $\ldots . . . . . . . . .7979$

7.1 Control variable bounds . . . . . . . . . . . . . . . . . . . . . . . . 88

8.1 Kriging model trend functions . . . . . . . . . . . . . . . . . . . 103 
8.2 Comparison of DACE model fitting characteristics with various searching algorithms using the initial TE-end plate CFD database averaged over 5 independent runs . . . . . . . . . . . . . . . . 110

8.3 Chosen search algorithm parameter settings . . . . . . . . . . . . 111

8.4 Comparison of DACE model fitting characteristics with varying nugget value using the initial TE end-plate CFD database and DE algorithm

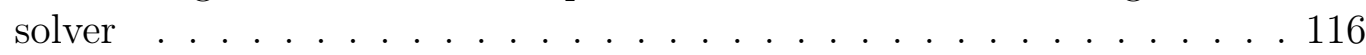

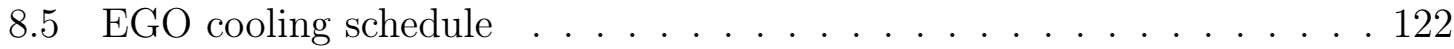

8.6 Summary of test functions used in EGO algorithm validation. . . . . . . 124

8.7 Comparison of optimizer performance metrics against four different test functions . . . . . . . . . . . . . . . . . . 125

8.8 Summary of relevant literature on DACE model stopping criteria . . . 127

8.9 Temporal requirements for the TE end-plate optimization using relevant DACE model literature with $k=3$. . . . . . . . . . . . . . . . . 128

9.1 Final selected designs . . . . . . . . . . . . . . . . . . . . . . . . . . 134

10.1 Test facility instrumentation errors (Endress and Hauser Group $[2020$, Louw, 2015) . . . . . . . . . . . . . . . . . . . 140

10.2 Experimental fan performance characteristics of trailing edge end-plate designs compared to fan datum evaluated at each design's operational flow rate for a $6 \mathrm{~mm}$ tip clearance . . . . . . . . . . . . . . . . 145

10.3 Maximum experimental test measurement uncertainty (valid for $4.12 \mathrm{~m}^{3} / \mathrm{s}$ $\left.\left.<V<16.38 \mathrm{~m}^{3} / \mathrm{s}\right)\right] \ldots \ldots \ldots \ldots \ldots \ldots \ldots \ldots$

10.4 Maximum experimental test measurement error vs end-plate design performance improvement over fan datum . . . . . . . . . . . . 150

A.1 Fan specifications (Wilkinson et al. $[2019)]$. . . . . . . . . . . . . . . . 164

A.2 $\quad$ Scale M-fan specifications for various tip clearances $\left.\left[N_{m}=722 \mathrm{rpm}\right]\right]$. 165

A.3 Scale M-fan design specifications . . . . . . . . . . . . . . . 165

B.1 Mesh density sensitivity of the scale M-fan domain at the $14.68 \mathrm{~m}^{3} / \mathrm{s}$ calculated design flow rate of Wilkinson et al. $[(2019)$ for a $0 \mathrm{~mm}$ tip clearance. . . . . . . . . . . . . . . . . . . 168

B.2 Outlet length sensitivity of the scale M-fan domain at the $14.68 \mathrm{~m}^{3} / \mathrm{s}$ calculated design flow rate of Wilkinson et al. $[(2019)$ for a $0 \mathrm{~mm}$ tip clearance. . . . . . . . . . . . . . . . . . . . . . . . . 169

B.3 Inlet length sensitivity of the scale M-fan domain at the $14.68 \mathrm{~m}^{3} / \mathrm{s}$ calculated design flow rate of Wilkinson et al. $(2019)$ for a $0 \mathrm{~mm}$ tip clearance . . . . . . . . . . . . . . . . . 169 
B.4 Tip clearance mesh density sensitivity of the scale M-fan domain at the $14.68 \mathrm{~m}^{3} / \mathrm{s}$ calculated design flow rate of Wilkinson et al. $(2019)$ for a $6 \mathrm{~mm}$ tip clearance . . . . . . . . . . . . . . . 171

F.1 Numerical forces acting on the optimized TE end-plate design evaluated

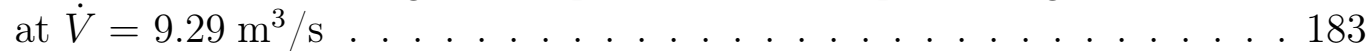

F.2 Material properties of biaxial $\left(+/-45^{\circ}\right)$ carbon fabric/epoxy composite 185

F.3 Optimized TE end-plate von Mises stress and carbon fabric/epoxy yielding stress comparison . . . . . . . . . . . . . 187

G.1 Pressure transducer calibration trend data . . . . . . . . . . . . . . . 189

G.2 Torque transducer calibration trend data . . . . . . . . . . . . . . . . . 191

G.3 Proximity sensor calibration trend data . . . . . . . . . . . . . . . . . . 193

H.1 Averaged initial zero reading measurement data of the datum fan . . . 194

H.2 Averaged initial no load measurement data of the datum fan . . . . . . 195

H.3 $\quad$ Averaged final zero reading measurement of the datum fan . . . . . . . . 195

H.4 Averaged final no load measurement of the datum fan . . . . . . . . . . . 195

H.5 Initial and final ambient air measurement data of the datum fan . . . . 195

H.6 Averaged measurement data of the datum fan evaluated at the $8^{\text {th }}$ volumetric flow rate . . . . . . . . . . . . . . . 196

H.7 Corrected measurement data of the datum fan evaluated at the $8^{\text {th }}$ volumetric flow rate . . . . . . . . . . . . . . 196

H.8 Fan performance characteristics of the datum fan evaluated at the $8^{\text {th }}$ volumetric flow rate . . . . . . . . . . . . . . . . 199 


\section{Nomenclature}

\begin{tabular}{|c|c|c|}
\hline Variables & & \\
\hline$A$ & Area & {$\left[\mathrm{m}^{2}\right]$} \\
\hline$D$ & Diameter & {$[\mathrm{m}]$} \\
\hline$E$ & Modulus of elasticity & {$\left[\mathrm{kg} / \mathrm{m} \cdot \mathrm{s}^{2}\right]$} \\
\hline$F$ & Force & {$[\mathrm{N}]$} \\
\hline$h$ & Height & {$[\mathrm{m}]$} \\
\hline$I$ & Turbulence intensity, Moment of inertia & {$\left[\%, \mathrm{~kg} \cdot \mathrm{m}^{2}\right]$} \\
\hline$k$ & Turbulent kinetic energy per unit mass & {$[\mathrm{J} / \mathrm{kg}]$} \\
\hline$l$ & Length & {$[\mathrm{m}]$} \\
\hline$M$ & Moment & {$[\mathrm{N} \cdot \mathrm{m}]$} \\
\hline$m$ & Mass & {$[\mathrm{kg}]$} \\
\hline$N$ & Rotational speed . . & {$[\mathrm{rev} / \mathrm{min}]$} \\
\hline$P$ & $\ldots .$. & {$[\mathrm{W}]$} \\
\hline$p$ & Pressure & {$\left[\mathrm{kg} / \mathrm{m} \cdot \mathrm{s}^{2}\right]$} \\
\hline$R$ & Radius, Specific gas constant . . . . & {$[\mathrm{m}, \mathrm{J} / \mathrm{kgK}]$} \\
\hline$r$ & Radial distance & {$[\mathrm{m}]$} \\
\hline$R M S E$ & Root-Mean-Square Error . . . . . . & {$[-]$} \\
\hline$T_{\text {shaft }}$ & Shaft torque & {$[\mathrm{N} \cdot \mathrm{m}]$} \\
\hline$t, \mathrm{t}$ & Thickness, Time . . . . . . . . & {$[\mathrm{m}, \mathrm{s}]$} \\
\hline$U$ & Absolute velocity & {$[\mathrm{m} / \mathrm{s}]$} \\
\hline$u$ & Blade tangential velocity & {$[\mathrm{m} / \mathrm{s}]$} \\
\hline$\dot{V}, V$ & Volumetric flow rate, Axial velocity & {$\left[\mathrm{m}^{3} / \mathrm{s}, \mathrm{m}\right]$} \\
\hline$v$ & Absolute vorticity, Displacement . & {$[\mathrm{Hz}, \mathrm{m}]$} \\
\hline$W$ & Relative velocity . . & {$[\mathrm{m} / \mathrm{s}]$} \\
\hline
\end{tabular}




\section{Greek symbols}

\begin{tabular}{|c|c|c|}
\hline$\beta$ & Exit trajectory angle & {$\left[{ }^{\circ}\right]$} \\
\hline$\epsilon$ & Turbulent dissipation rate & {$[\mathrm{J} / \mathrm{kg} \cdot \mathrm{s}]$} \\
\hline$\zeta$ & Total loss coefficient, Setting angle & {$\left[-,{ }^{\circ}\right]$} \\
\hline$\eta$ & Efficiency & {$[-]$} \\
\hline$\theta$ & DACE hyperparameter & {$[-]$} \\
\hline$\kappa$ & Condition number . . & {$[-]$} \\
\hline$\lambda$ & Geometric scaling factor, Eigenvalue & {$[-,-]$} \\
\hline$\mu$ & Dynamic viscosity, Mean value. . . & {$[\mathrm{kg} / \mathrm{m} \cdot \mathrm{s},-]$} \\
\hline$\rho$ & Density & {$\left[\mathrm{kg} / \mathrm{m}^{3}\right]$} \\
\hline & Standard deviation, Stress & {$\left[-, \mathrm{kg} / \mathrm{m} \cdot \mathrm{s}^{2}\right.$} \\
\hline & Tip clearance .... . & {$[\mathrm{m}]$} \\
\hline & Rotational speed, Turbulent specific dissipation rate & {$[\mathrm{rad} / \mathrm{s}, \mathrm{Hz}]$} \\
\hline
\end{tabular}

\section{Dimensionless numbers}

\begin{tabular}{|c|c|c|}
\hline$C_{L}$ & Coefficient of lift & {$[-]$} \\
\hline$C_{\mu}$ & Turbulence model constant & {$[-]$} \\
\hline Co & Courant number & {$[-]$} \\
\hline$C p$ & Static pressure coefficient & {$[-]$} \\
\hline$F, f$ & Function ... & {$[-]$} \\
\hline$g$ & Inequality constraint . . & {$[-]$} \\
\hline$H_{\text {norm }}$ & Normalized helicity . . . & {$[-]$} \\
\hline$k$ & Dimensionality & {$[-]$} \\
\hline$n$ & Number . . . & {$[-]$} \\
\hline$R e$ & Reynolds number & {$[-]$} \\
\hline Ro & Rossby number & {$[-]$} \\
\hline$v, \mathrm{v}$ & Nugget, Poisson's ratio & {$[-]$} \\
\hline$w$ & Weighting parameter . . & {$[-]$} \\
\hline$x / l_{c}$ & Fraction of chord . . . . & {$[-]$} \\
\hline$y+$ & Wall distance & {$[-]$} \\
\hline
\end{tabular}

\section{Superscripts}
-
Averaged value
$*$
Optimized value 


\section{Subscripts}

\begin{tabular}{|c|c|}
\hline 01 & Stagnation inlet location \\
\hline 2 & Static outlet location \\
\hline bell & Bellmouth \\
\hline$c$ & Chord \\
\hline$d$ & Design, Dynamic \\
\hline$e p$ & End-plate \\
\hline$i$ & Inlet, Inner \\
\hline$L$ & Lift \\
\hline$L B$ & Lower bound \\
\hline$l$ & Lower \\
\hline$M$ & Moment \\
\hline$m v b$ & Multiple vortex breakdown \\
\hline$m$ & Model \\
\hline$m c$ & mid-chord \\
\hline $\max$ & Maximum \\
\hline $\operatorname{mix}$ & Mixing \\
\hline$n / e$ & Numerical/Experimental \\
\hline$o$ & Outlet, Outer \\
\hline plen & Plenum chamber \\
\hline span & Spanwise \\
\hline$T L F$ & Tip leakage flow \\
\hline$T L V$ & Tip leakage vortex \\
\hline$t-s$ & Total-to-static \\
\hline$U B$ & Upper bound \\
\hline$v$ & von Mises \\
\hline vte & Variable thickness end-plate \\
\hline$Y$ & Yield \\
\hline$\infty$ & Free stream \\
\hline
\end{tabular}

\section{Abbreviations}

ANN Artificial Neural Networks

AoA Angle of Attack

ACC Air-Cooled Condenser 
ACHE Air-Cooled Heat Exchanger

AMI Arbitrary Mesh Interface

CAD Computer Aided Design

CFD Computational Fluid Dynamics

COP Centre of Pressure

CSP Concentrated Solar Power

CT Constant thickness

DACE Design and Analysis of Computer aided Experiments

DE Differential Evolution

DoE Design of Experiment

DP Design Point

EGO Efficient Global Optimization

EI Expected Improvement

GA Genetic Algorithm

GAMG Geometric Agglomerated Algebraic Multigrid Solver

GP Gaussian Process

HE Heat Exchanger

IGES Initial Graphics Exchange Specification

LB Lower Bound

LE Leading Edge

LES Large Eddy Simulations

LHS Latin Hypercube Sampling

LHSMDU Latin Hypercube Sampling with Multidimensional Uniformity

LOO Leave-One-Out

MCS Maximum Cell Size

MLE Maximum Likelihood Estimation

MOMP Multi Objective Multi Point

MOO Multi Objective Optimization

N.A. Not Applicable or Not Achievable

OF OpenFOAM

P3DM Periodic Three-Dimensional Model

PS Pressure Side

RANS Reynolds Averaged Navier Stokes

RBF Radial Basis Function 
RR Rotor Region

RSM Response Surface Model

SCF Spatial Correlation Function

SHM SnappyHexMesh

SIMPLE Semi-Implicit Method for Pressure Linked Equations

SM Surrogate Model

SOO Single Objective Optimization

SS Suction Side

SST Shear Stress Transport

STL Stereolithography

TE Trailing Edge

TLF Tip Leakage Flow

TLV Tip Leakage Vortex

UB Upper Bound

VSD Variable Speed Drive

VTK Visualization ToolKit 


\section{Chapter 1}

\section{Introduction}

\section{$1.1 \quad$ Background}

Air-cooled heat exchangers (ACHEs) find extensive application throughout the oil and gas industry as well as the power generation industry. According to Kröger (2004), these types of heat exchangers (HEs) offer a significant advantage over competing technologies (such as wet-cooled systems) as they minimize water consumption and provide for a cost effective alternative. This makes them more favourable for use in dry, arid regions where water is not readily available and the cost to make use of such resources would be uneconomical.

ACHEs work by rejecting heat to the atmosphere through the movement of large volumes of air over a multitude of HE bundles. These HE bundles then carry the working fluid through the system and as such transfer heat to the air through convection. Air flow is achieved through the use of an array of large industrial scale axial flow fans (some up to 10 meters in diameter) which create the needed pressure differential in order to motivate the movement of such large volumes of air.

The size requirement of these fans is necessary due to the poor heat transfer properties of air. This makes for a large initial capital outlay when making use of such technology. As such, this motivates further fan development, as a small improvement in fan performance could lead to a large reduction in cost, as both the size and number of fans required might be reduced.

Recent trends make use of high volume flow rate, low pressure rise fan designs. This requires fans to be highly efficient in order to perform effectively under the unique operating conditions commonly encountered (Wilkinson, 2017). From 2016 to 2018, the European Union funded the MinWaterCSP project which forms part 
of the larger Horizon 2020 research and innovation program. This project was aimed at reducing water usage in concentrated solar power (CSP) plants while maintaining cycle efficiency in order to reduce the cost of making use of such technology (MinWaterCSP, 2017).

The project produced the M-fan, a rotor only axial flow fan designed to meet the design specifications as set out by the project. However, during scale experimental testing the M-fan was found unable to meet the required design specifications. A possible cause for this could be related to tip clearance effects (as suggested by Wilkinson et al., 2019) as the M-fan was initially designed with a zero tip clearance and as such did not take tip clearance effects into account. As a result, the current study will focus on improving the performance of the M-fan through introduction of a design which considers the tip region.

Due to the desire for maximum fan performance enhancement, there lies an inherent difficulty in developing a design which achieves this objective across a broad range of operating conditions (as is commonly encountered during the fan's operation). As such, optimization of the conceptual design will be conducted with the aim of achieving an overall better result. Optimization is a routinely used tool in the field of turbomachinery for aerodynamic performance enhancement. However, the complex nature of the flow near the blade tip may result in model inefficacies during implementation thereby leading to a sub-optimal solution. Therefore, along with the relevant literature, algorithm selection will be performed using a comparative analysis of various models to determine the most appropriate for use on the current optimization problem.

Engineers make use of numerical modelling or computational fluid dynamics (CFD) to assess the performance of axial flow fans in order to reduce costly experimental testing. However, licensing restrictions associated with commercial software packages means that open-source alternatives such as OpenFOAM (OF) have become increasingly popular.

Although free, one drawback of open-source software is that it does not offer the type of support and development of commercial software packages. This leaves uncertainty into the capabilities of such software, especially when modelling more complex geometries. However, as the finite volume discretization of the solution domain (the meshing process) is most critical in determining accurate results, users of OF generally make use of OF solvers in combination with commercial meshing packages in order to reduce the uncertainty, while at the same time reducing cost. 
However, this still requires the need for costly licensing fees of commercial meshing software. Therefore, this study will also consider the use and validation of available open-source meshing software to become completely independent of commercial software packages.

\subsection{Test fan}

The test fan used in the present study is that of the M-fan. The specifications of the full-scale fan are shown in Table 1.1.

\begin{tabular}{ll} 
Table 1.1: M-fan specifications & Wilkinson \\
\cline { 2 - 2 } Design specifications & \\
\hline Number of blades $[-]$ & 8 \\
Blade root setting angle $\left[^{\circ}\right]$ & 34 \\
$D_{\text {rotor }}[\mathrm{m}]$ & 7.315 \\
Hub-to-tip diameter ratio $\left[D_{\text {hub }} / D_{\text {rotor }}\right]$ & 0.29 \\
Rotor tip clearance $\left[\tau / D_{\text {rotor }}\right]$ & 0.004 \\
Volumetric flow rate $\left[\mathrm{m}^{3} / \mathrm{s}\right]$ & 333 \\
Rotational speed $[\mathrm{rpm}]$ & 151 \\
\hline
\end{tabular}

The M-fan was specifically designed for application in ACHEs and as such has been both numerically and experimentally tested at model scale during its development. Therefore, as the current study involves a numerical analysis of the M-fan, this thesis will only consider the development of the scale model of the M-fan in order to validate against experimental data. The scaling procedure for the M-fan is described in Appendix A. 


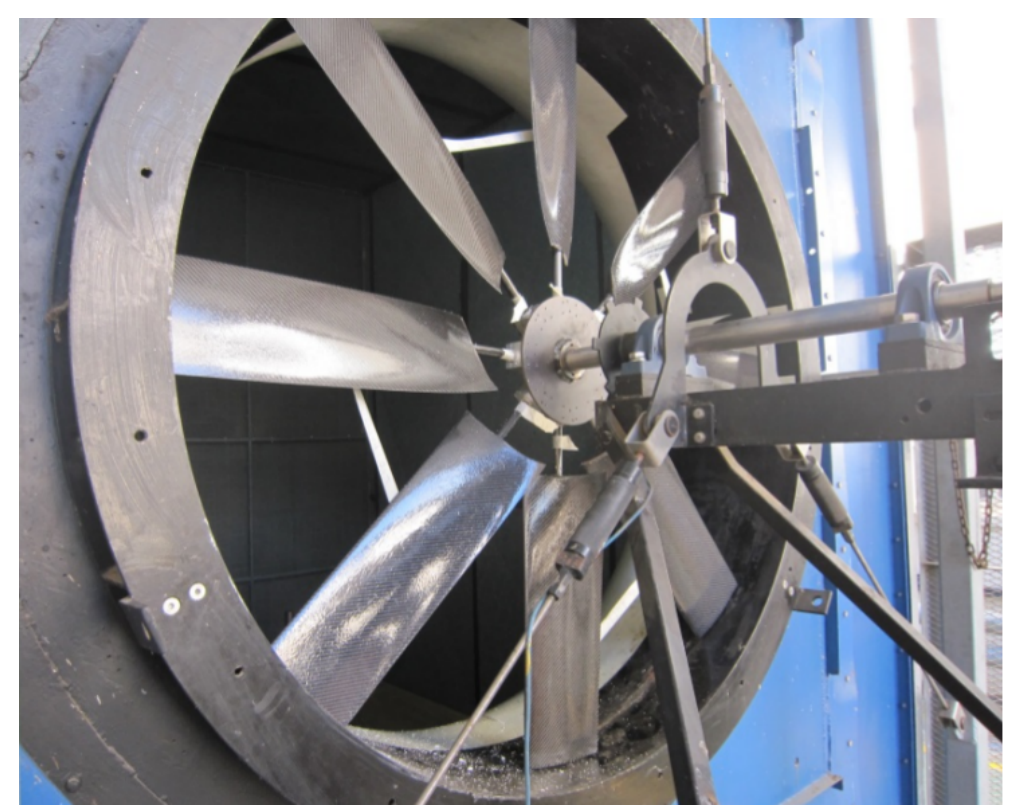

Figure 1.1: Scale M-fan experimental assembly (Wilkinson et al., 2018)

\subsection{Research objectives and methodology}

From the given background, several objectives were identified for this thesis, namely:

- Development of a numerical model using only open-source software. With the desire for cost reduction, a method whereby full access to open-source software could become available using currently available alternatives would be of significant benefit.

- Numerical assessment of the effects of tip clearance on fan performance. Since the effect of tip clearance on fan performance has been noted to be a potential performance inhibitor, the effect is investigated to gain insight into possible cause and mitigation. This is compared with previous works to develop a better understanding of the flow phenomena present around the blade tip region and its effect on fan performance.

- Development of a design to mitigate the effects of tip clearance and improve fan performance. Based on previously gathered information within literature and this study, various design procedures for the control and mitigation of tip clearance effects are introduced. Numerical simulations provide insight into the dominating features and expected performance enhancement of the various designs. 
- Design optimization using design of experiments method. Once analyzed, the most promising design is selected for numerical optimization using the design of experiments method (DoE). Identification of the most influential geometric parameters on fan performance is made based on the findings of the optimization process.

- Experimental testing. Verification of the numerical results is carried out through experimental testing of various end-plate designs. The scale M-fan is modified and tested according to the ISO 5801 type A fan test facility set-up using various tip end-plate configurations.

Figure 1.2 illustrates the current study's workflow breakdown. As can be seen, the literature review forms the basis of the study. Therefore, the current thesis is organized as follows: Chapter 2 conducts a study into current literature on tip clearance effects and its mitigation. Thereafter, Chapters 3 and 4 consider the numerical meshing and simulating as well as the validation thereof. Chapter 5 conducts the numerical post-processing of the scale M-fan at various tip clearances to investigate the effect of, and gain insight into, the relevant flow phenomena. Using these analyses, along with the relevant literature, Chapter 6 introduces two design procedures to mitigate the deleterious effects of tip clearance. Thereafter, Chapter 7 evaluates and defines the most appropriate design for the current implementation. This information is then used in the optimization routine presented in Chapter 8 . Finally, the numerical and experimental evaluation of the optimization process is presented in Chapters 9 and 10, respectively. Lastly, Chapter 11 concludes the study with a summary of all results obtained and conclusions drawn. 


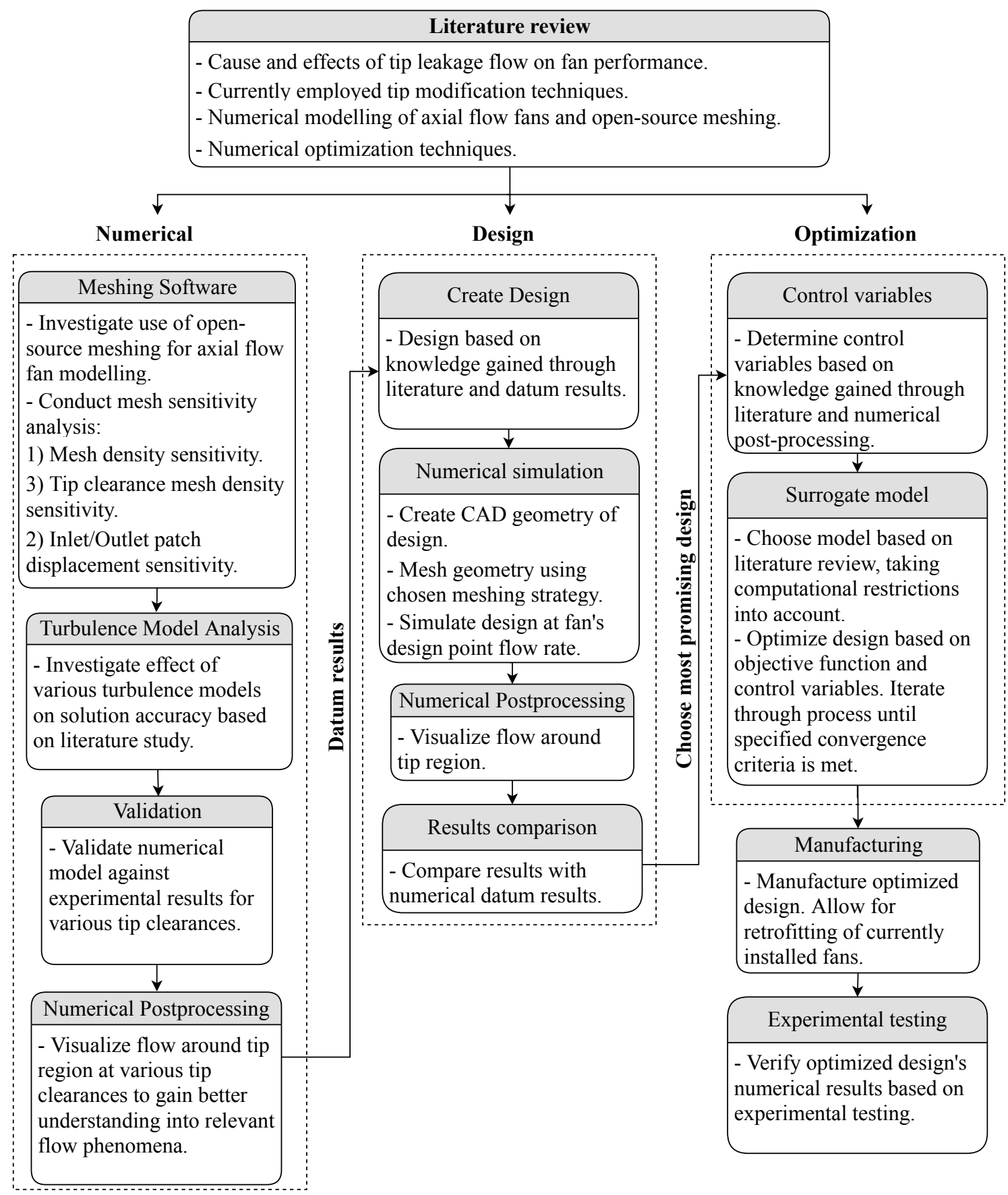

Figure 1.2: Work flow breakdown 


\section{Chapter 2}

\section{Literature review}

A prior knowledge of the basic principles and flow phenomena that affect axial flow fan performance through tip leakage flow must be attained before the design procedure can commence. This is in order to gain understanding of the losses produced so that the proposed design could control such effects. The following sections are divided as such: description of relevant flow phenomena, its effects on fan performance, current modification techniques employed to control such flow phenomena and a study on current open-source modelling and meshing techniques. Finally, various optimization techniques and details of their definition are also investigated.

\subsection{Tip leakage flow}

The tip clearance in an axial flow fan is defined as the distance between the blade tip and fan casing. This clearance is necessitated to allow movement between the rotating and stationary components as well as to allow the adjustment of the blade stagger angle.

During the fan's operation, a pressure differential exists between the blade pressure side (PS) and suction side (SS) near the blade tip. This pressure differential induces a localized flow, known as tip leakage flow (TLF), which flows through the tip gap from the high pressure to the low pressure side of the blade. A visual representation can be seen in Figure 2.1, which shows the blade rotation from right to left and the TLF can be seen flowing from left to right over the blade tip. 


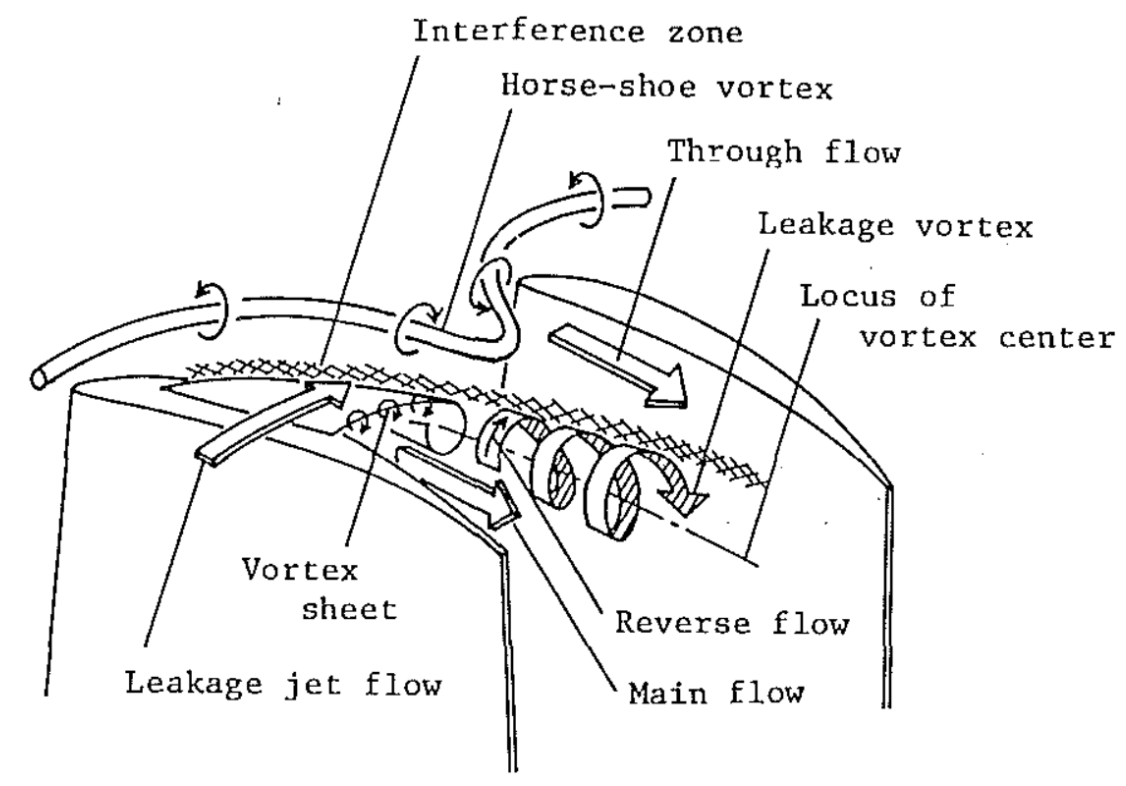

Figure 2.1: Tip leakage flow visualization (Inoue and Kuroumaru, 1989)

This leakage flow, which is of a different magnitude and direction to that of the main flow, rejoins the main flow on the low pressure blade side. The skewed merging angle of the two incoming air flows cause the TLF to roll up and form a vortex, known as the tip leakage vortex (TLV), which grows in size further along the blade chord as the TLF supplies energy to the TLV. Downstream of the blade, the TLV begins to break down and dissipate into the main flow. The TLF (and subsequent TLV formation) is associated with having a detrimental effect on fan pressure and fan efficiency (Cumpsty, 2004).

\subsection{Tip effects on fan performance}

\subsubsection{Tip leakage loss}

In order to quantify the effects of TLF on fan performance, a method by which the quantification of the local losses near the blade tip needs to be introduced. As noted by Cumpsty (2004), the four major loss sources are:

- Drag at solid surfaces

- Mixing

- Shock losses 
- Shear work

Of these, the loss due to mixing is most appropriate in describing the TLF loss. This is because the vortex created inevitably has to dissipate into the main flow to once again reach a uniform state. It is in achieving this that the major losses are to be expected (Cumpsty, 2004). The intense shearing that takes place in the thin layer between the two high-speed air streams (of differing magnitude and direction) is the principle mechanism behind the high loss associated with TLF (Cumpsty and Storer, 1991). By considering a two-dimensional cascade, Cumpsty (2004) describes a total loss coefficient due to mixing as

$$
\xi_{\text {mix }}=\frac{p_{01}-p_{03}}{\frac{1}{2} \rho U^{2}}
$$

where $p_{01}$ and $p_{03}$ represent the stagnation pressure of the cascade upstream and downstream, respectively.

In a different study investigating the effects of TLF during the experimental testing of a linear cascade, Cumpsty and Storer (1991) found that by maintaining a constant pressure on the blade pressure side, it is only the pressure on the blade suction side that changes with tip clearance. Cumpsty and Storer (1991) propose this to be the controlling mechanism in the chord-wise distribution of the TLF.

\subsubsection{Tip clearance}

Venter and Kröger (1992) reported that a reduction in tip clearance leads to an increase in fan static pressure rise and fan static efficiency across all flow rates. Venter and Kröger (1992) found the effect of tip clearance to be linearly related to both fan static pressure rise and volumetric flow rate at a constant rotational speed. This suggests that minimizing the tip clearance reduces the effect of the TLF, thereby reducing the associated pressure losses. However, if the tip clearance is removed entirely, a corner separation between the casing and blade suction surface would form (Adamczyk et al., 1993). This introduces another source of loss which would yet again reduce both fan pressure and fan efficiency. Therefore, a tip clearance exists which is large enough to minimize or even negate corner separation losses, while also being small enough to minimize the loss incurred due to the TLF.

In a single-stage compressor experimental test, Lakshminarayana (1970) found the optimal tip clearance to lie between 1 and 1.5 percent of chord for rotors (Cumpsty, 2004). Inoue et al. (1986) found the optimum to be less than 1 percent of pitch. However, in application small tip clearances are not always possible and larger 
than optimal clearances are normally implemented. The consensus in literature is that large tip clearances have a detrimental effect on fan performance and should be reduced as far as practical.

Using the VDI fan test code of Verein Deutscher Ingenieure (1966), Venter and Kröger (1992) noted that the improvements due to a reduction in the tip clearance in scale model tests underestimated the performance increase in the full scale tests. The authors suggest this to be related to the boundary layer thickness and pressure differential across the fan rotor. This means that the effect of tip clearance is more pronounced in large scale fans and a small improvement in scale model tests can have a more significant effect in larger scale fans.

\subsubsection{Tip geometry}

\section{Tip edge}

The tip geometry has a substantial effect on the development of the TLV, particularly on the swirl and axial velocity distribution of the vortex in the very early wake (Green, 1995). During an experimental study evaluating the effect of round and square edged tip geometries on the formation of the TLV, Giuni (2013) found that a square edged tip produced multiple unsteady and intense secondary vortex structures while a round edged tip did not force the TLF into separation and produced a more axisymmetric vortex structure. The unsteadiness of these secondary vortices would cause greater mixing between the TLF and the surrounding fluid. The secondary vortices have been described to be weaker than that of the main vortex which allows for a smoother interaction during the rolling up process of the wake sheet around the main vortex (Giuni, 2013).

Figure 2.2 shows the entering TLF separating at the pressure side of the blade tip, which then subsequently forms a separation bubble. This separation bubble causes a vena contracta effect within the tip gap which aids in reducing the effective tip clearance, thereby reducing the TLF. 


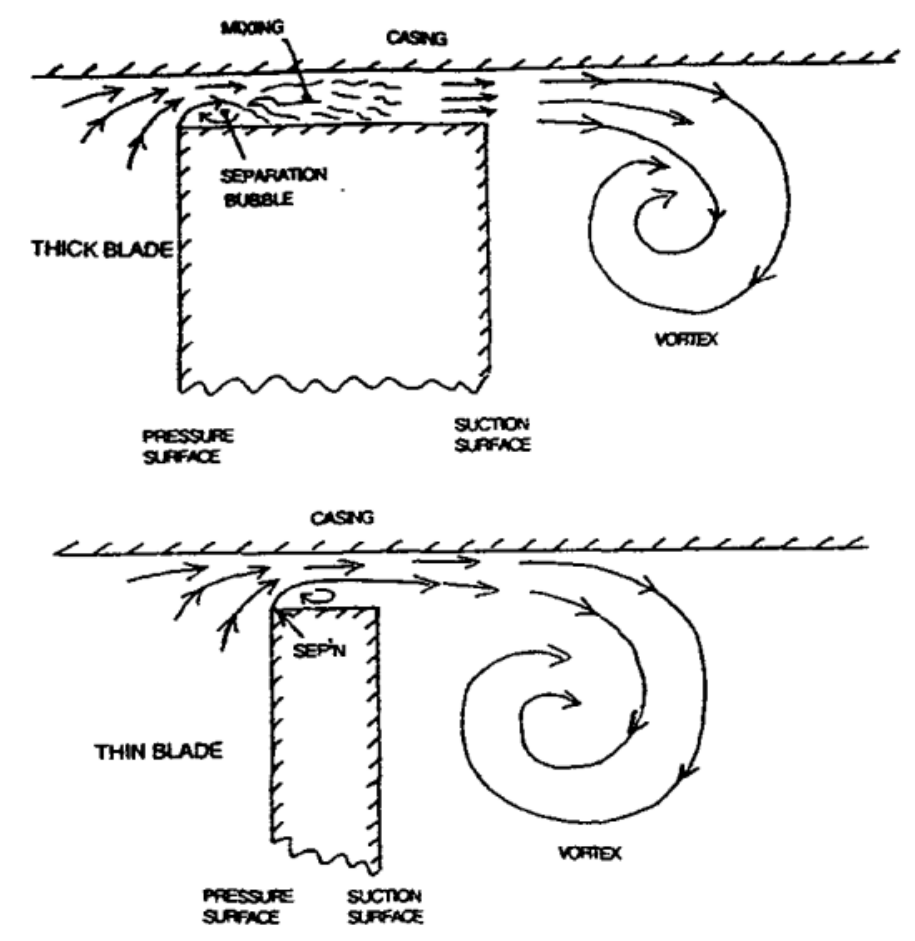

Figure 2.2: Tip leakage flow visualization with variable blade thicknesses (Denton, 1993)

As noted by both Rains (1954) and Denton (1993), the entrance loss (commonly integrated into a contraction coefficient) of the incoming air flow is related to the radius of the tip edge. Denton (1993) also noted that the flow up to the throat of the jet is almost isentropic and is not greatly influenced by the component of velocity along the blade chord. This supports the assumption made by both Rains (1954) and Corsini and Sheard (2013) that the TLF acts in a normal direction relative to the blade camber line.

\section{Tip thickness}

Denton (1993) determined that if the tip clearance is less than 25 percent of the blade tip thickness, the TLF will mix out within the tip gap with a consequent increase in static pressure and entropy. The premature mixing of the TLF within the tip gap may result in the reduction of the TLF's normal velocity component. This would result in a smoother diffusion of the TLF into the main flow, thereby resulting in reduced overall mixing losses. If the tip clearance is greater than 40 percent of the blade's tip thickness, the TLF will not reattach within the tip gap (Storer, 1991), as shown in the bottom of Figure 2.2. 


\subsubsection{Boundary layer effects within the tip region}

During an experimental analysis, Volino (2014) found the endwall boundary layer thickness upstream of the blade row to have a significant effect on the total pressure loss and secondary velocity fields within a linear cascade of high pressure turbine blades in a low speed wind tunnel. By increasing the endwall boundary layer thickness, Volino (2014) found the total pressure loss to increase while also reducing the TLF. This was also found to result in a weaker TLV which did not penetrate as deep into the pressure side flow. This could be as a result of an increased vena contracta effect caused by the increased boundary layer thickness within the tip region. Consequently, this suggests an optimal inlet boundary layer thickness to exist for a specific tip clearance which results in the minimum total pressure loss being incurred.

\subsection{Tip modification techniques}

From literature, there are two categories by which tip modification techniques are employed to control TLF in turbomachinery, namely: active and passive control techniques. The former makes use of dynamic means such as wall casing treatments and fluid injection to alter the interacting flow and thereby improve performance. The latter makes use of static means such as changes in blade configuration or tip modifications to achieve the same desired outcome. Mitchell and Delery (2001) noted that the adoption of dynamic control devices has an inherent difficulty in identifying the characteristic frequencies of the vortical structures for the positional adjustment and intensity of such devices. Consequently, the current thesis will only focus on passive control techniques.

During an initial study, Corsini et al. (2006) investigated the effects of improving the performance and aero-acoustic characteristics of a subsonic axial flow fan through the use of a passive control device that modified the blade tip geometry. The introduction of this feature would potentially mean an increase in fan performance at moderate tip clearances, which would reduce the associated disadvantages thereof. Corsini et al. (2006) used a constant thickness end-plate design (denoted TF end-plate in Figure 2.3 ) which thickened the blade tip locally by a factor of 3:1 with respect to maximum blade thickness. This dimension was chosen as the reference radial dimension of the TLV to be controlled. The value of which is estimated to be in the range of $0.1-0.2$ blade span, as shown in former studies on rotors of axial compressors (Inoue et al., 1986) and fans (Corsini et al., 2006).

Corsini and Sheard (2007a) found the numerical results of the TF end-plate design 
to show a decrease in the datum fan's performance in terms of both fan static pressure rise and fan efficiency. They postulated this to be due to a vortex bursting phenomenon present at the blade tip. According to the experimental results at the fan's $7 \mathrm{~m}^{3} / \mathrm{s}$ design point flow rate, the fan static pressure rise was found to decrease by up to 6.4 percent. However, an increase of up to 2.0 percentage points in fan static efficiency is apparent with the addition of the end-plate design when compared to the datum fan.

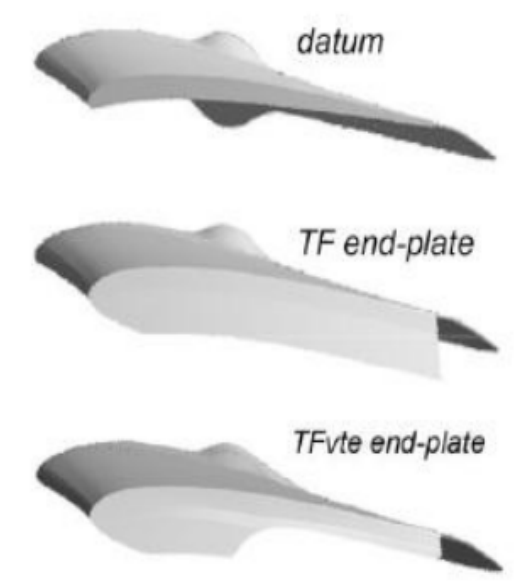

a)

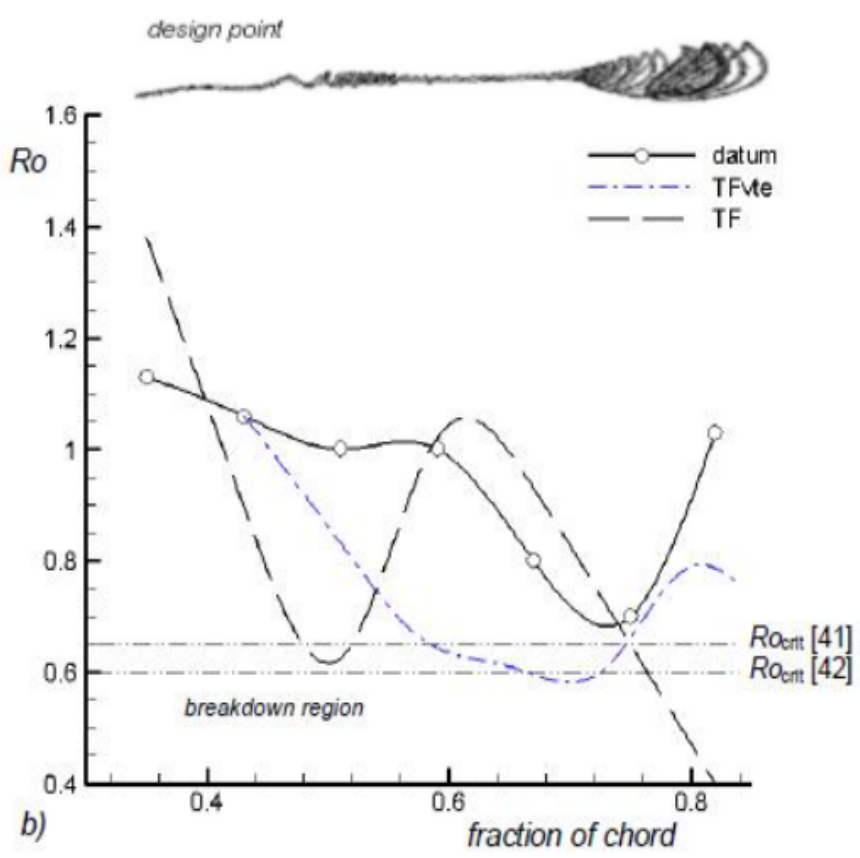

Figure 2.3: a) Various tip end-plate designs with b) their respective chord-wise vortex Rossby number (Ro) evolutions at the fan's $7 \mathrm{~m}^{3} / \mathrm{s}$ design operating point (adapted from Corsini et al., 2010)

Corsini and Sheard (2007a) consequently presented a variable thickness end-plate design (denoted TFvte in Figure 2.3a) which aimed at preventing the TLV bursting phenomenon found in the TF end-plate design. According to Corsini and Sheard (2007a), this is achieved by enhancing the vortex's near axis swirl and introducing waviness into its trajectory by using the momentum transfer of the TLF. The rationale behind this design is based on the definition of the breakdown criteria of Spall et al. (1987) who used a locally defined Rossby number $(R o)$, or inverse swirl ratio, to define the onset of vortex breakdown. The local Rossby number is expressed by: 


$$
R o=\frac{V}{r \omega}
$$

where $r$ is defined as the radial distance from the vortex axis where the swirl velocity is a maximum, $\omega$ is the rotation rate of the TLV near the vortex centre, and $V$ is defined as the axial velocity at $r$. Using a critical $R o$ number range, from 0.6 for wing tip vortices with 'bubble' or 'spiral' type vortex breakdown (Garg and Leibovich, 1979) to 0.64 for axisymmetric vortex breakdown in swirling air flows (Uchida et al., 1985), Corsini and Sheard (2007a) defined a variable tip end-plate thickness distribution based on a simplified law for the tip clearance pressure drop and a safe chord-wise gradient Ro number.

As illustrated in Figure 2.3 $\mathrm{b}$, the origin of the bursting and subsequent breakdown of the TFvte end-plate vortex corresponds well to the critical Ro number range as suggested in the literature. This seems to support the validity for the use of the Ro number in vortex breakdown determination. The experimental results of the TFvte design show a reduction in fan static pressure rise by up to 4.3 percent, yet, an increase in fan static efficiency of up to 3.0 percentage points at the design operating point when compared to the datum fan. These results suggest superior performance of the TFvte end-plate design when compared to that of the TF endplate design.

Further design improvements gave rise to the multiple vortex breakdown TFmvb design of Corsini and Sheard (2013) (shown in Figure 2.4a). Using the knowledge gained from previous designs, the TFmvb end-plate design increases the end-plate thickness where a decrease in momentum transfer is desired. The rationale behind the design is based on the sequential break down of the TLV into multiple smaller vortices along the blade chord which is thought to further reduce losses. This could hold some truth in that the smaller vortices would be more dominated by the main flow thereby decreasing their exit trajectory angle so that they become more aligned with the main flow direction. This would mean a smoother diffusion of the TLF into the main flow thereby reducing the incurred mixing losses. 


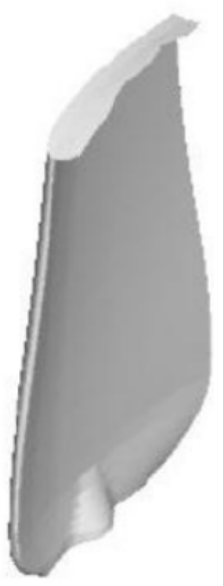

a)

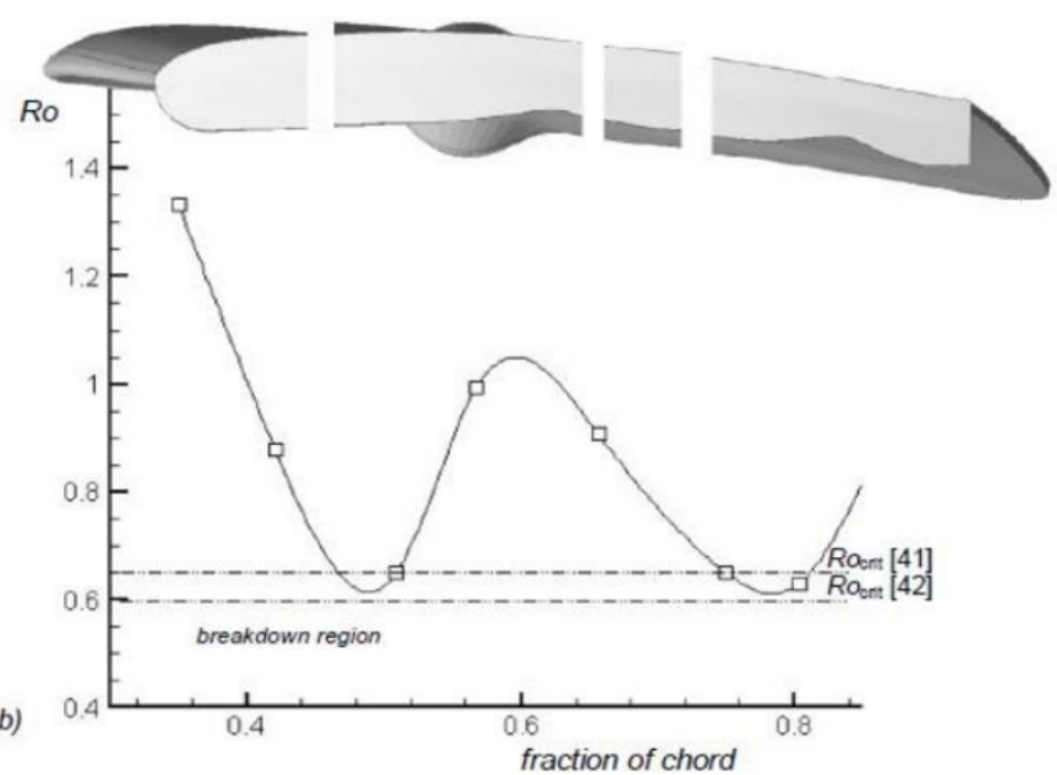

Figure 2.4: a) TFmvb end-plate design with b) chord-wise vortex Rossby number evolution at the fan's $7 \mathrm{~m}^{3} / \mathrm{s}$ design operating point (adapted from Corsini and Sheard, 2013)

As shown in Figure 2.4 b, the TFmvb end-plate design contains two points of vortex breakdown, located around 0.5 and 0.8 chord fractions. The Ro distribution is blade specific, as noted by Corsini and Sheard (2013), yet it is unclear as to the influence of the position and distribution of such breakdown regions along the chord for the TFmvb end-plate design. The TFmvb end-plate design is found to increase the datum fan's total pressure rise by 7 percent while maintaining a similar efficiency at design point (Corsini and Sheard, 2013).

Figure 2.5 presents the performance curves of all the end-plate designs mentioned thus far. All the end-plate designs improve the fan's total efficiency at lower than peak efficiency flow rates; with a subsequent reduction in efficiency at higher flow rates. The TFmvb end-plate shows the greatest improvement in fan total pressure rise, however, gives the lowest fan total efficiency at higher than design flow rates. 

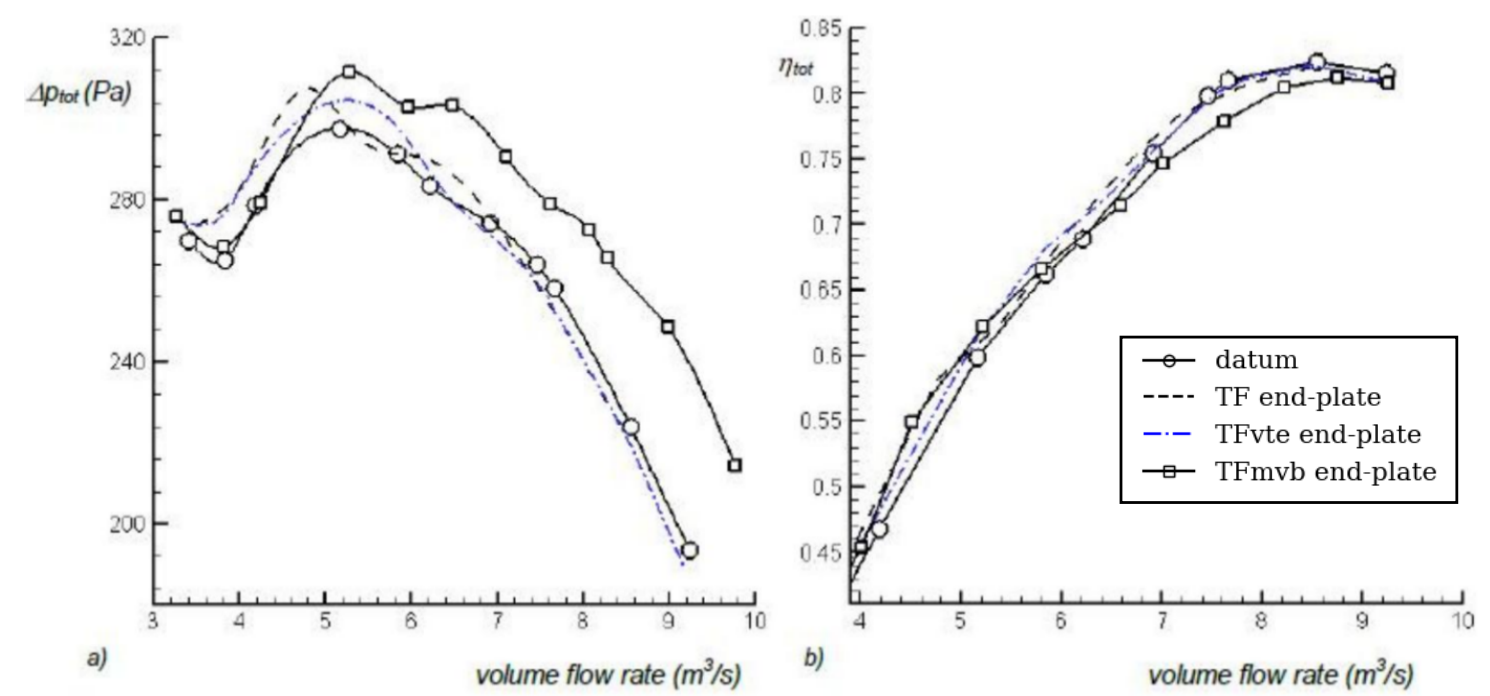

Figure 2.5: a) Experimental fan total pressure and b) total efficiency performance curves (adapted from Corsini and Sheard, 2013)

Additionally considered passive control devices include that of blade sweep ( $\mathrm{Vad}$, 2008), squealer/grooved/slotted tips (Ye et al., 2015, Jiang et al., 2015, Ye et al., 2017), tip flanges (Zhang et al., 2014) and sinusoidal leading edge serrations (Corsin. et al. 2015). However, due to a lack of data or design applicability these devices were not further investigated for use in the current study.

\section{$2.4 \quad$ Numerical analysis}

\subsubsection{Three-dimensional modelling of an axial flow fan}

Three-dimensional modelling of a turbomachine can be considered either fully three-dimensional (in which case the full geometry is modelled) or periodic (where only a periodic section of the geometry is modelled). The latter being far less computationally expensive.

Considering the domain upstream and downstream of the rotor section, the majority of the publications studied in this thesis make use of either an open inletoutlet or extended annular duct configuration (Corsini et al., 2006, Zhang et al. 2014; Louw, 2015; Ye et al., 2015; Liu et al., 2016; Wilkinson, 2017). The former models the rotor section with an expanded inlet and outlet domain to represent the open atmosphere, similar to that of an ISO 5801 type A test set-up (International Organization for Standardization, 2007). The latter simply makes 
use of extended hub and casing lengths upstream and downstream of the rotor section at a constant radius. The advantage of the extended annular duct configuration when compared to that of the open inlet-outlet configuration would be a reduction in computational time as the mesh and solver requirements are significantly reduced (Louw, 2015).

\subsubsection{Open-source meshing}

Since it is desired to limit the use of commercial software packages, open-source meshing software is used in the current study. Currently available open-source meshing software packages include GMSH, cfMesh, Meshlab, Blender, and OFs blockMesh and snappyHexMesh (SHM) utilities. However, an additional requirement would the ability to easily incorporate various designs into the meshing procedure with minimal input from the user. For this reason only automatic meshing software packages will be considered. The following sections discuss the most relevant open-source meshing software alternatives.

\section{cfMesh}

CartesianMesh is a semi-automated, hexahedral dominant, three-dimensional mesh generator within the cfMesh program. Given an input STL (Stereolithography) file and specified maximum cell size, the program creates a three-dimensional mesh aligned to a cartesian coordinate system. The program attempts to optimize for orthogonality while selecting cells to modify in order to accommodate the given geometry.

The entire process is semi-automated in that it only requires a geometrically closed geometry file (yet not necessarily watertight as the program is designed to tolerate poor quality geometry files (Juretić, 2015) ) and a set of user specified refinements to produce a mesh. The user specified refinements accommodate specific surface and zone refinements which increase mesh density in areas of particular interest.

Refinements are specified by either the cell size for a particular surface patch or a refinement region of a particular shape and size (square, cone etc). The program then creates a mesh of predominantly structured hexahedral cells with polyhedra in the transition regions between the cells of differing size (Juretic, 2015). Edge refinement is specified in a similar manner by specifying the edges in a separate VTK (Visualization ToolKit) format.

cfMesh supports wall layer cell addition; however, the open-source version can only add layers within the first cell layer on a particular patch. This restricts the total 
number of layers grown and overall layer thickness to the surface patch refinement level.

Figure 2.6 gives an example of the program's meshing procedure. The red circles indicate the edge refinement procedure while different surface refinement levels can be seen by comparing the surrounding surfaces. Note how the addition of the wall layer cells within the figure are limited to the first cell layer over the wall patches.

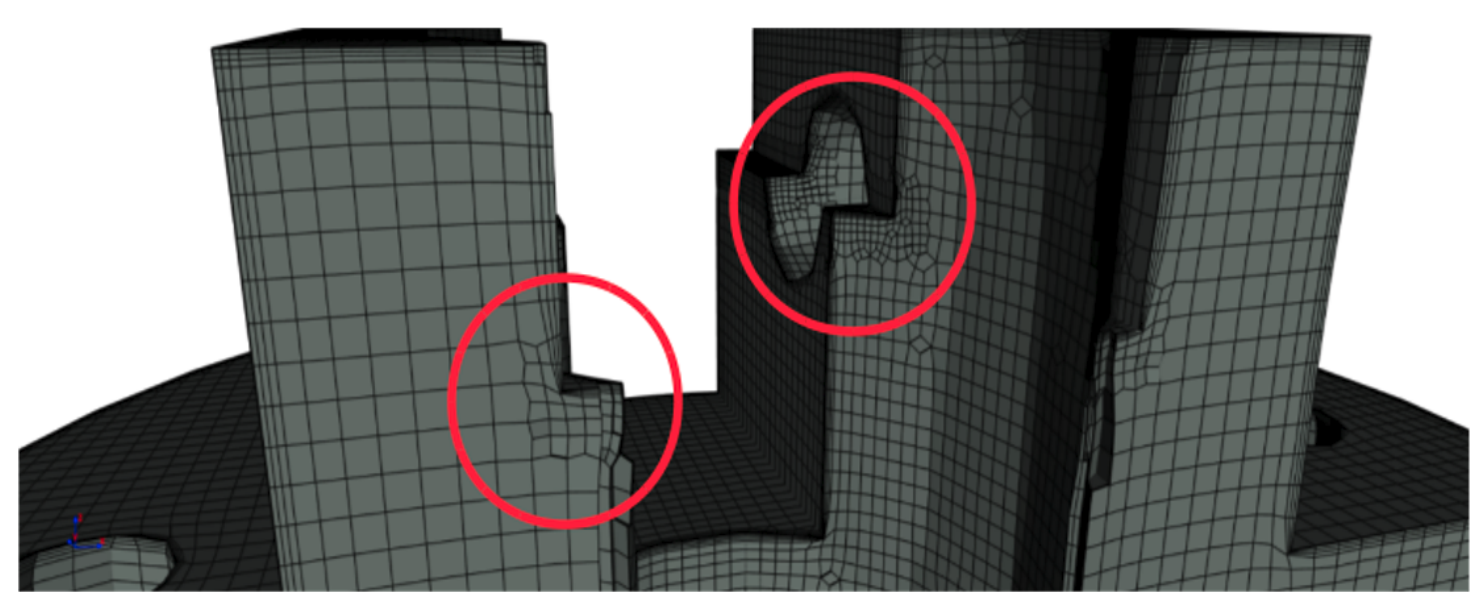

Figure 2.6: Detail of cfMesh mesh generation process (Juretić, 2015)

\section{SnappyHexMesh}

The OF SHM utility is a semi-automatic, three-dimensional mesh generator containing hexahedral and split-hexahedral cells. SHM makes use of triangulated surface geometries (in STL or Wavefront Object format) during mesh generation. The program forms part of the OF software package and as such is designed with the software's solving capabilities in mind. As shown in Figure 2.7, SHM makes use of a four stage method in its mesh generation, namely: a) initial mesh creation, b) castellation, c) snapping and d) layer addition. 


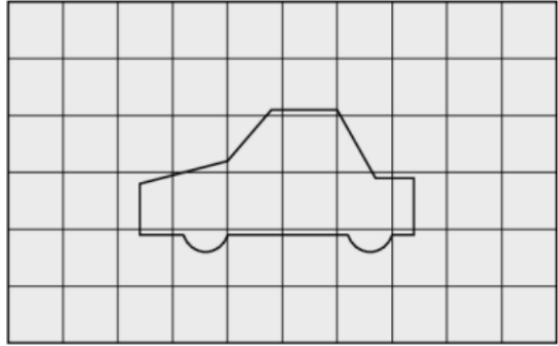

a)

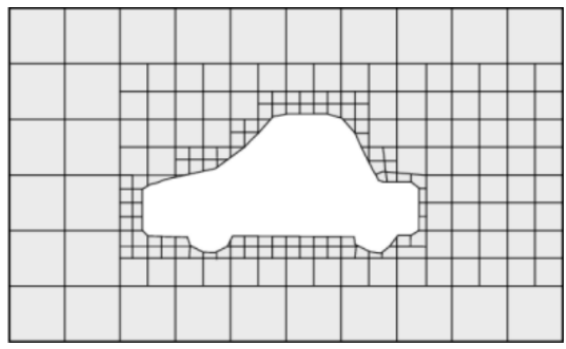

c)

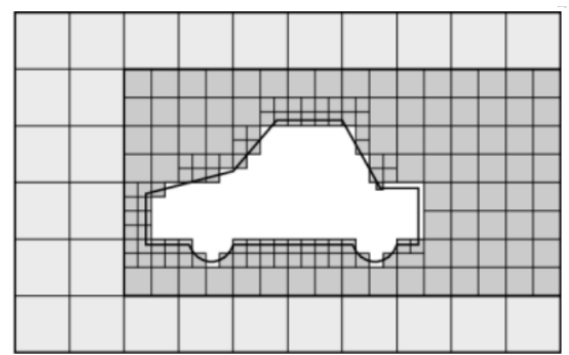

b)

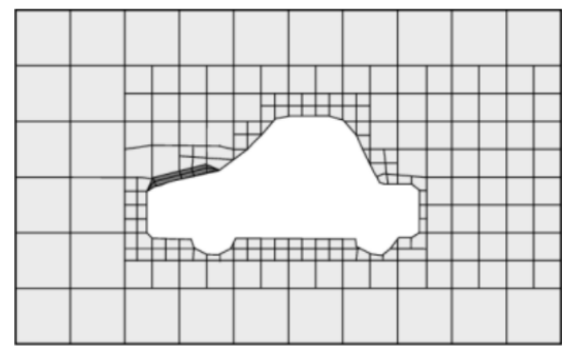

d)

Figure 2.7: SnappyHexMesh meshing procedure: a) initial mesh creation, b) castellation, c) snapping, and d) layer addition (OpenCFD Ltd, 2018)

\section{Stage 1: Initial mesh creation}

During the initial mesh creation stage, the OF blockMesh utility is used to create a pure hexahedral background mesh of specified overall dimension and edge division (which determines maximum cell size) that surrounds the given STL file. In a similar manner to cfMesh, the user can then specify surface, region, and edge refinements using levels. Increasing the number of refinement levels simply increases the number of times the specified cells are split.

Edge files are created using the OF utility surfaceFeatureExtract which differentiates between edges by a user specified minimum include angle. The created files have an eMesh format which means that most computer aided design (CAD) software packages are unable to import and edit this type of file. This makes it difficult to specify specific edge refinements.

\section{Stage 2: Castellation}

After stage 1, SHM removes any cells that do not have 50 percent or more of their volume within the enclosed geometric region, as shown in Figure 2.7b. The region of cells that are retained is selected depending on a user specified location. 


\section{Stage 3: Snapping}

Once stage 2 is complete, the mesh is left highly castellated around the geometry's edges. The snapping stage of SHM involves moving any vertices that lie outside of the enclosed region towards the closest surface. The program then relaxes the internal mesh to accommodate the moved vertices while simultaneously limiting their displacement (or adding new vertices) to prevent violation of the mesh quality parameters. This iterative procedure continues until mesh quality is satisfied or the maximum number of iterations is reached. An example is shown in Figure 2.7k.

\section{Stage 4: Layer addition}

Finally the layers are added using a bottom up approach as shown in Figure $2.7 \mathrm{~d}$. By specifying the patch name, first layer thickness and number of layers, the program attempts to grow the cells from the surface of the patch outward. The program simply adds the new cells over the specified patch while displacing and deforming the internal mesh to accommodate the newly added cells. The program iterates through this process, while attempting to conform to a user defined set of quality parameters. It should be noted that if the quality parameters are too strict or the total layer thickness too large, the program will reduce layer growth or not even grow the layer cells at all.

\subsubsection{Numerical models}

All but one of the publications studied make use of steady-state simulations through solving of the Reynolds Averaged Navier Stokes (RANS) equations, the remaining study making use of large eddy simulation (LES). However, as noted by Inoue and Furukawa (2002), the use of the RANS equations are considered an effective investigation tool for vortical structure detection. This is advantageous as the mesh and computational requirements of RANS simulations are far less than those of LES.

Of the various turbulence models available, the majority of publications studied involving numerical modelling make use of some variant of the linear $k-\epsilon$ model of Launder and Spalding (1974). Consequently there exists extensive validation records on this family of models, particularly with regards to incompressible aerofoil and turbomachinery applications. Of the studies conducted on tip end-plates, Corsini et al. (2006) make use of a low-Reynolds variant of a non-linear $k-\epsilon$ model, which attains good agreement with experimental results. 
A number of publications also make use of some variant of the $k-\omega$ model of Menter et al. (2003), which has been described to be specific for aeronautical applications and therefore gives good predictions for flows across aerofoils (Louw, 2015). In a comparison of turbulence models which include both the $k-\epsilon$ and $k-\omega$ variants, Louw (2015) found the transitional $k-k l-\omega$ model of Walters and Cokljat (2008) and the transitional shear stress transport (SST) model of Menter et al. (2003) to produce the best results when compared to experimental data.

The type of turbulence model used and the meshing considerations go hand-inhand in producing an accurate solution. Standard models such as that of the $k-\epsilon$ or $k-\omega$ model, although easy to implement and provide for fast convergence, overpredict the turbulence properties at stagnation points and require near wall corrections (i.e wall functions). It is the extent of these near wall corrections that determine the type of solving required (that being high-Reynolds or low-Reynolds approaches) and hence affect the mesh requirements.

If a model requires near wall corrections, then standard wall functions are used to model the wall boundary layer within the log-law region. Other models such the $k-\omega$ SST model (which require no such near wall corrections) can be used in a low-Reynolds approach to integrate through the wall boundary layer. A low-Reynolds approach is more computationally expensive than that of the highReynolds approach yet provides for a more accurate representation of the boundary layer.

\subsection{Optimization techniques}

In the field of turbomachinery, numerical modelling is often used to facilitate the development of conceptual designs in order to reduce the need for expensive prototyping. However, solving these types of engineering problems with computer models can become computationally expensive and time-consuming. In such an instance, the use of design optimization is impractical and places a limit on the number of objective function evaluations, thereby reducing the overall accuracy of the process. A solution to this problem is to simulate by means of metamodelling to emulate the statistical input-output relationship at a reduced computational cost (Durantin et al. 2016). The following sections discuss various techniques commonly used during metamodelling. 


\subsubsection{Surrogate models}

Metamodels, also referred to as surrogate models (SMs) or response surface models (RSMs), are able to solve problems of the "black-box" type at a reduced number of function evaluations. These functions are termed black-box as they are associated with difficult real-world optimization problems where the actual shape of the function is usually not known a priori. The algorithms used in SMs generally follow a two step process, namely: 1) fit or train the model based on a finite data set of input control variables with their corresponding response values, and 2) search the response surface for an optimal point.

Various SM algorithms exist within literature, with each algorithm's performance being situationally dependent. As such, the selection of the SM used in this investigation is discussed in Chapter 8 . However, as noted in the same chapter, the chosen SM requires effective searching of the model hyperparameters in order to accurately fit to the data to then be able to better determine the optimal solution. Therefore, the following section investigates appropriate searching algorithms that may be used in conjunction with the chosen SM.

\subsubsection{Search algorithms}

Many of the practical optimization problems faced by engineers include multimodal and nonlinear objective functions with nonconvex and disconnected regions. Solving these types of global optimization problems can become challenging. Stochastic global optimization techniques offer an alternative to this problem. Instead of finding and comparing all local optima to identify the global optimal solution, candidate solutions are sampled and evaluated to approximate the global optimum in a probabilistic way (Ali et al., 2005$)$. This is recommended as optimization of such problems can become computationally expensive thereby making optimization impractical.

Likewise with the selection of an appropriate SM, the selection of an appropriate searching algorithm is also situational dependent. As a result, and following the "no free lunch" theorem of Wolpert and Macready (1997), two evolutionary algorithms are considered for further investigation. The following sections discuss details of the chosen algorithms as well as the rationale behind their selection.

\section{Genetic Algorithm}

In the various publications studied involving the shape and design optimization of turbomachinery, the genetic algorithm (GA) was found to be the most pre- 
dominant stochastic global optimization algorithm employed Ashihara and Goto, 2001; Chen and Yuan, 2008; Kim et al., 2011; Pei et al., 2016; Li et al., 2017). In a comparison between several stochastic optimization algorithms tested against 56 global optimization test problems, Ali et al. (2005) found the GA to perform best provided that the number of function evaluations are sufficiently high. However, the GA takes large optimization steps which could lead to inefficiencies when locating global optimal solutions (Ashihara and Goto, 2001).

Consequently, the continuous GA of Haupt and Ellen Haupt (2004) is chosen for comparative analysis in the current investigation. A continuous variant is selected because it is more logical to represent variables as floating-point numbers which happens to make the algorithm inherently faster than its binary counterpart (Haupt and Ellen Haupt, 2004).

The general steps of the continuous GA are as follows:

1. Initialization: Randomly generate an initial population $S$ of size $N_{\text {pop }}$ within the feasible region.

2. Selection: Select $N_{\text {keep }}=0.5 N_{\text {pop }}$ best individuals in $S$ as parents based on their fitness.

3. Mating ("crossover"): Pair two selected parents for mating to produce two offspring, which replace $N_{\text {pop }}-N_{\text {keep }}$ worst points (lowest fitness) in $S$ to give $S^{\prime}$. This method uses a single point crossover which is randomly chosen for both parents. At the point of crossover, a blending method is used to introduce new genetic material as described in Equation 2.2.

$$
\begin{array}{r}
p_{\text {new } 1}=p_{m \alpha}-\beta\left[p_{m \alpha}-p_{d \alpha}\right] \\
p_{\text {new } 2}=p_{d \alpha}+\beta\left[p_{m \alpha}-p_{d \alpha}\right]
\end{array}
$$

where $\mathrm{m}$ and $\mathrm{d}$ subscripts differentiate between the mom and dad of the selected parents. Standard crossover is performed between the two parents which includes the newly added points.

4. Mutation: Mutate $N_{\text {mut }}=\mu\left(N_{\text {pop }} \times N_{\text {var }}\right)$ points by randomly selecting a variable from a randomly selected individual in $S^{\prime}$ for each mutation, ensuring mutations are within the feasible region.

5. Update $S$, set $g=g+1$ and repeat from step 2 until specified convergence criteria is met. 
Considering the above steps, it can be seen that the algorithm has one main userdefined parameter, the mutation rate $\mu$. In the case of the continuous GA, a value of $20 \%$ is chosen for this parameter (Haupt and Ellen Haupt, 2004).

\section{Differential Evolution}

Another population-set based algorithm considered is the differential evolution (DE) algorithm proposed by Storn and Price (1997). This is a relatively new algorithm which is gaining increasing popularity within the turbomachinery community (Mueller et al., 2013, De Maesschalck et al., 2016, Bergh, 2018). This algorithm is more deterministic than that of the GA yet has a comparably similar structure. In a recent study involving the comparison between the two algorithms for the design optimization of a single stage turbine rotor, Bergh $(2018)$ found the $\mathrm{DE}$ algorithm to converge in fewer iterations with a lower root-mean-square error $(R M S E)$ than that of the GA.

The general steps of the DE algorithm are as follows:

1. Initialization: Randomly generate an initial population $S$ of size $N_{p o p}$ vectors $(\mathbf{x})$ within the feasible region.

2. Mutation: Perform a mutation for each vector using a randomly sampled and scaled vector difference combination. Equation 2.3 shows how the mutant vector, $\mathbf{v}_{i, g}$, is created:

$$
\mathbf{v}_{i, g}=\mathbf{x}_{r 0, g}+F \cdot\left(\mathbf{x}_{r 1, g}-\mathbf{x}_{r 2, g}\right)
$$

where $r$ signifies that the vector is random and $g$ the generation number. This equation represents a rand/1 strategy which refers to the use of a random base vector with a single vector difference during mutation.

3. Crossover: Perform a discrete recombination of each vector on a componentwise basis by crossing the current vector $\mathbf{x}_{i, g}$ with the mutant vector $\mathbf{v}_{i, g}$ to create a trial vector $\mathbf{u}_{i, g}$ :

$$
\mathbf{u}_{i, g}= \begin{cases}\mathbf{v}_{j, i, g} & \text { if } \operatorname{rand}_{j}(0,1) \leq C r \text { or } j=j_{\text {rand }} \\ \mathbf{x}_{j, i, g} & \text { otherwise }\end{cases}
$$

where $i$ and $j$ represent the current member in the population and the current component of that vector, respectively. This equation refers to a binomial distribution ("bin") or uniform crossover. 
4. Selection: Perform a greedy selection between the trail vector $\mathbf{u}_{i, g}$ and current vector $\mathbf{x}_{j, i, g}$ by evaluating the fitness of each. Replace the current vector in $S$ if found to deliver a higher (worse) objective function value:

$$
\mathbf{x}_{i, g+1}= \begin{cases}\mathbf{u}_{i, g} & \text { if } f\left(\mathbf{u}_{i, g}\right) \leq f\left(\mathbf{x}_{i, g}\right), \\ \mathbf{x}_{i, g} & \text { otherwise }\end{cases}
$$

5. Repeat from step 2 until specified convergence criteria is met.

As shown above, the two user-defined parameters are that of the mutation factor, $F \in(0,1+)$, and the crossover ratio (also known as the "recombination rate"), $\mathrm{Cr} \in[0,1]$. These control the rate at which mutation occurs and determine the fraction of recombined component values, respectively. As noted by Price et al. (2005), while there is no upper limit on the value of $F$, values are seldom selected greater than 1.0. With this in mind, consideration of step 2 reveals that the created vector can exceed the boundaries of the feasible domain. As such, bounding methods used to control these violating vectors are discussed in Section 2.7.1. The DE steps are illustrated in Figure 2.8 below. 

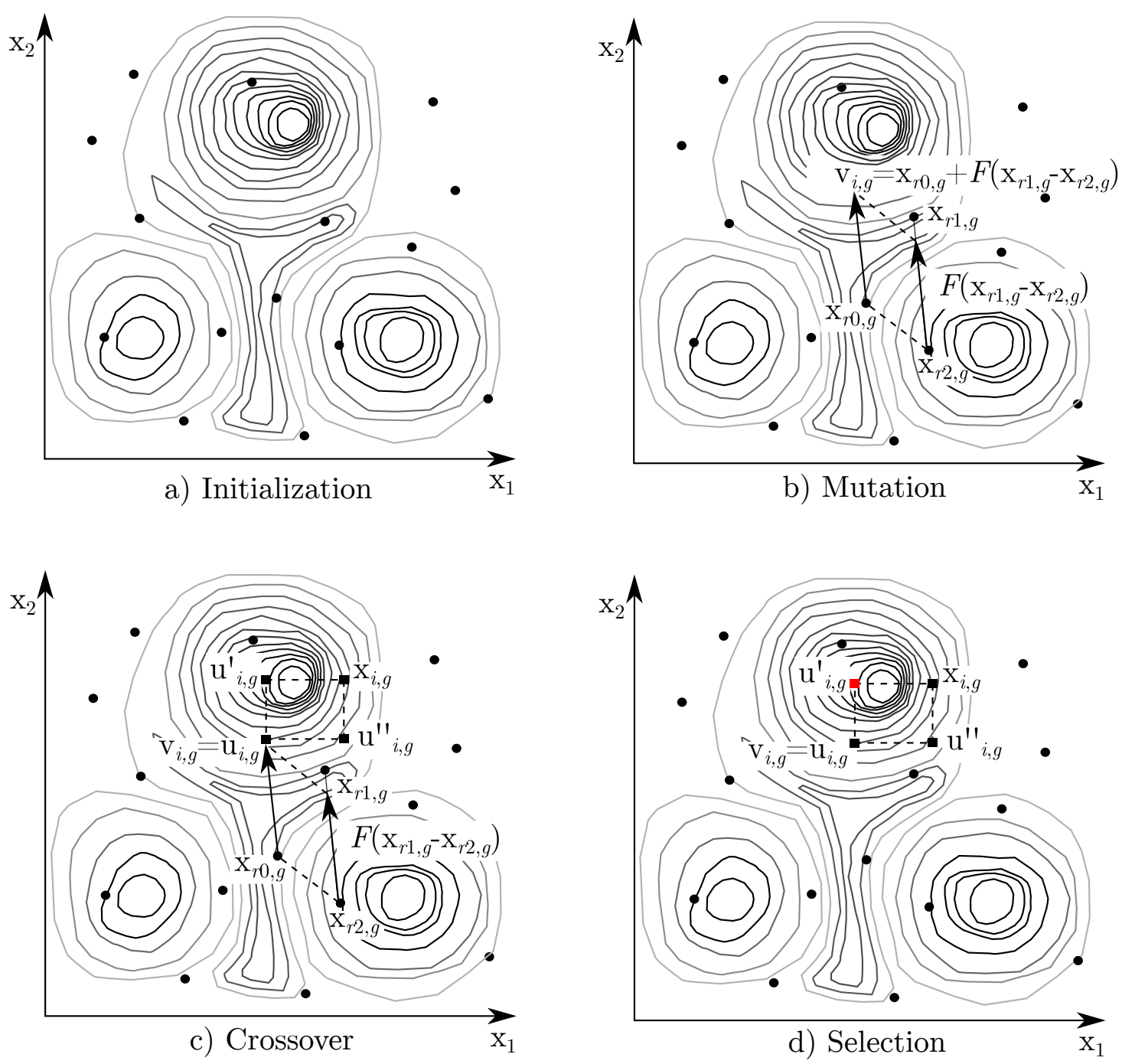

Figure 2.8: Illustration of the steps of the differential evolution algorithm

\subsection{Optimization objective function}

Definition of the optimization algorithm's objective function forms a critical step in the design procedure as it dictates the shape of the response surface and therefore has a direct influence on the final design. The standard form of the optimization problem is represented by finding the set of response variables $\mathbf{x}=\left(x_{1}, \ldots, x_{k}\right)$ that minimizes some objective function,

$$
\underset{x \in \mathbb{R}^{k}}{\operatorname{minimize}} f(x)
$$


subject to constraints:

$$
\begin{gathered}
x_{L B} \leq x_{i} \leq x_{U B},(i=1, \ldots, n), \\
h_{j}(x)=0,(j=1, \ldots, p), \\
g_{m}(x) \leq 0,(m=1, \ldots, q),
\end{gathered}
$$

where:

$f(x)$ is the objective function,

$x$ is the input control variable bound by an upper and lower limit of the feasible region $\left(x_{U B}\right.$ and $\left.x_{L B}\right)$,

$h(x)$ represents the set of equality constraints, and

$g(x)$ represents the set of inequality constraints.

If $p=q=0$, the problem is defined as unconstrained. Various techniques exist in order to enforce control over the optimization routine to ensure efficient searching through the design space when locating optima. The following sections describe some of the techniques commonly employed when formulating the optimization algorithm's objective function.

\subsubsection{Single-objective optimization}

Single-objective optimization (SOO) makes use of a single function in order to evaluate the performance of the optimizer. Ordinarily, this function is presented as a minimization problem (as given in Equation 2.6) but can easily be represented as a maximization problem by taking the negative of the former.

\subsubsection{Multi-objective optimization}

Multi-objective optimization (MOO) is the simultaneous optimization of more than one unique objective function in order to find the optimal solution that best compromises between competing objectives. In this instance, competing objectives form a hypersurface of equally optimal and non-dominated points within the feasible objective space to create the so-called Pareto-front. All solutions that make up this front are referred to as Pareto-optimal solutions. Since the determination of the entire continuous Pareto-optimal surface is practically impossible, a discrete set of Pareto-optimal points are commonly used to approximate the true distribution of the front (Price et al., 2005). Figure 2.9 illustrates an example of a non-dominated Pareto-front with its respective Pareto-optimal solutions. 


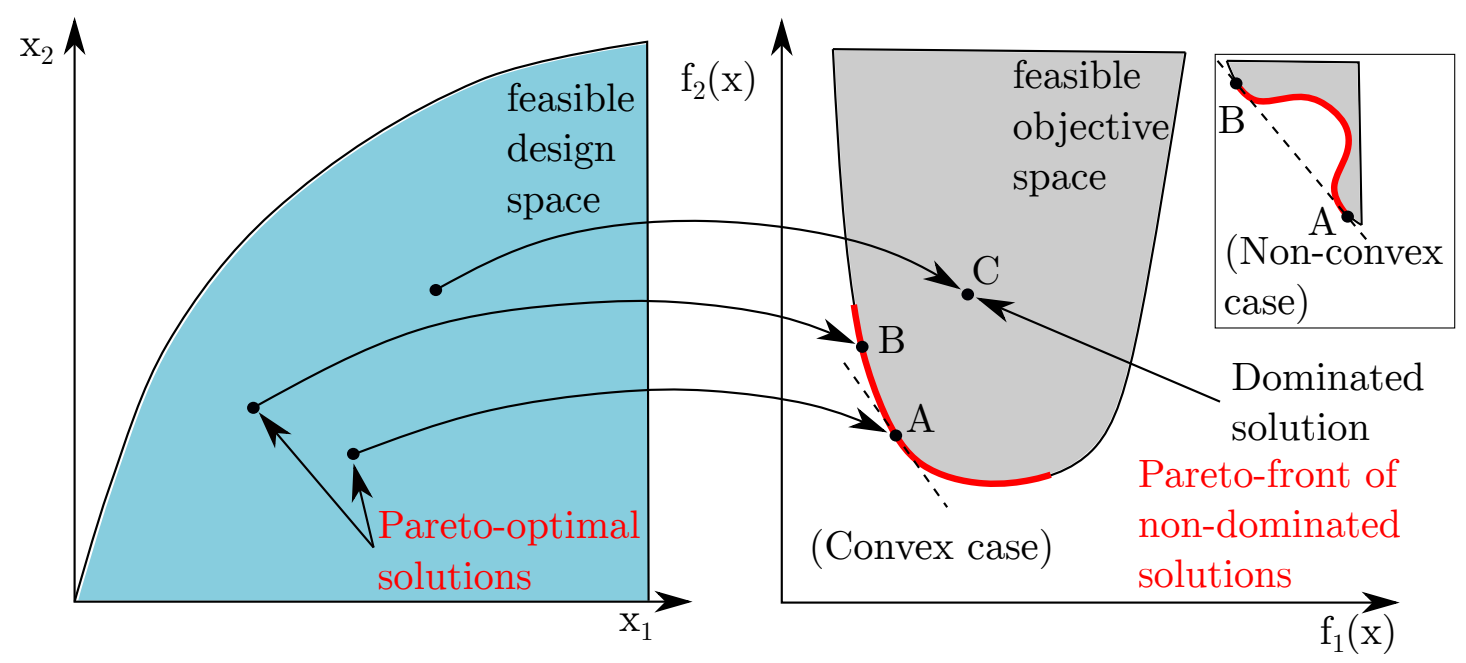

Figure 2.9: Multi-objective optimization (adapted from Sudhoff (2007))

One method that transforms a multi-objective optimization problem to consist of only a single objective function (which is more easily applied to the optimizer's searching algorithm) is that of the weighted sum method. This method adds a scalarized set of objective functions to formulate a single objective by using individual weightings. This is expressed mathematically by:

$$
f^{\prime}(\mathbf{x})=\sum_{m=1}^{M} w_{m} f_{m}(\mathbf{x})
$$

where:

$f^{\prime}(\mathbf{x})$ is the pseudo objective function,

$w_{m}$ are the individual weightings assigned to each objective function, and

$f_{m}(\mathbf{x})$ represents the individual objective functions.

Although easy to implement, the choice of the individual objective weightings may force the solution outside of the feasible objective space if chosen incorrectly, thereby leading to an incorrect Pareto-optimal solution. Moreover, as shown in Figure 2.9, this method is unable to find Pareto-optimal solutions in the case of non-convex objective spaces (Sudhoff, 2007).

An alternative method that is applicable to both convex and non-convex problems is that of the $\epsilon$-constraint method (Sudhoff, 2007). This method selects a single objective function to optimize (similar to that of SOO) and treats the remaining 
objective functions as additional constraints to be bound by a user-defined set of $\epsilon$ vectors. However, likewise with the weighted sum method, the $\epsilon$ vectors must be carefully selected in order to prevent non-optimal solutions.

\subsection{Optimization with constraints}

Most practical, real-world optimization problems involve finding the optimal solution while simultaneously satisfying one or more constraint functions (as introduced in Section 2.6). Constraints restrict the movement of trial vectors to be located within the feasible objective space. Figure 2.10 illustrates the difference between a constrained and unconstrained optimization problem.

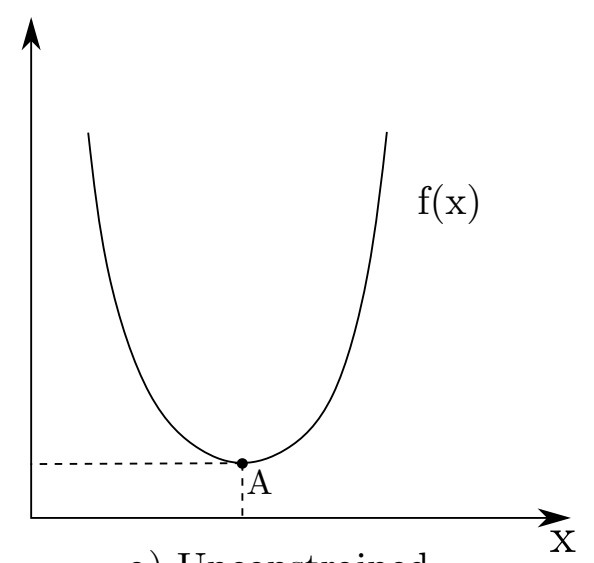

a) Unconstrained

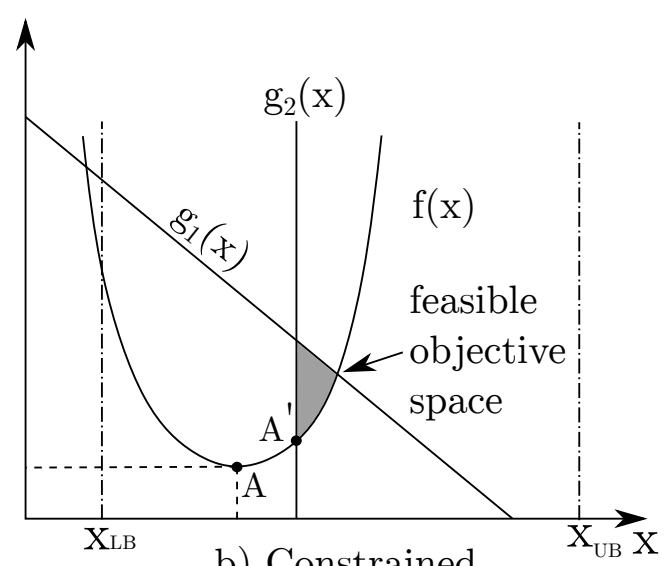

b) Constrained

Figure 2.10: Constrained vs unconstrained optimization problem

Figure 2.10a represents the ideal case where the optimum at point $A$ coincides with the functions global optimum. However, this is often not the case for many optimization problems as the optimal point may be located in non-feasible regions (possibly due to structural or manufacturing limitations). Figure 2.10b illustrates the same objective function, yet constrained by functions $g_{1}(x)$ and $g_{2}(x)$. In this scenario, the previously unconstrained optimal point $A$ is shifted to the constrained optimal point $A^{\prime}$ to lie within the feasible objective space. If formulated correctly, these constraints could potentially negate local minima thereby preventing premature convergence of the algorithm (Price et al. 2005$)$.

Various constraint handling techniques exist within literature, with each being better suited to handle specific constraints. For the sake of brevity, only boundary and inequality constraint handling techniques will be discussed. 


\subsubsection{Boundary constraints}

Boundary constraints represent restrictions on objects that relate to physical components or quantities which have natural bounds, for example: both length and mass must be non-negative (Price et al. 2005$)$. Although technically the same as inequality constraints, boundary constraints are separately mentioned here due to differences in the methods used. Price et al. (2005) discusses two distinct methods by which these types of constraints are handled, namely:

1. Penalty methods which attempt to motivate non-conforming trial vectors to feasible ones by modifying the objective function, and

2. Resetting schemes which modify out-of-bounds parameters to ensure that their trial vectors satisfy all constraints.

\section{Penalty methods}

The simplest of the more stringent penalty methods is that of the "brick wall" method. In this method, the objective function value of parameters that violate constraint bounds is replaced by a sufficiently high value to ensure that it will not be selected. However, bounds should be carefully selected when using this method as progress can be slowed down if the optimum is located near bounds (which can make convergence improbable). A derivative of this method is that of the adaptive penalty method. Unlike that of the brick wall method, this method increments the objective function value based on the magnitude and number of violating parameters.

\section{Resetting schemes}

As penalty methods do not tolerate out-of-bounds trial vectors, progress will be slow when feasible trial vectors are rare. In this instance, resetting schemes may offer an advantage as out-of-bounds parameters are modified to acceptable values when infeasible, thereby generating more solutions.

Of the resetting schemes, the two most commonly used methods are random reinitialization and bounce-back. The former, being the most unbiased of the methods, randomly initializes out-of-bounds parameters to lie within the feasible range. However, as parameter values are radically altered, progress can be disrupted for near bounds solutions (Price et al., 2005$)$. The latter method takes the progress of the solution into account by selecting the out-of-bounds parameters to randomly lie between a feasible base vector and the violating upper or lower parameter bound. This negates the previous issue of near bounds solutions as trial vectors are located even closer to violating bounds. 


\subsubsection{Inequality constraints}

Inequality constraints represent the set of functions that form the feasible objective space within which a solution must be found. Two main methods exist by which inequality constraints are handled, namely:

1. Penalty and barrier functions, and

2. Direct constraint handling.

\section{Penalty and barrier functions}

Similar to that of the adaptive penalty method for boundary constraints, penalty functions modify the objective function value based on constraint violations. A common approach used to incorporate these constraint violations is to simply combine the weighted sum of the penalty functions with the objective function to form a modified objective function, $F(\mathbf{x})$ :

$$
F(\mathbf{x})=f(\mathbf{x})+\sum_{m=1}^{M} w_{m} p_{m}(\mathbf{x})
$$

where:

$f(\mathbf{x})$ is the original objective function,

$w_{m}$ are the individual weightings assigned to each penalty function, and

$p_{m}(\mathbf{x})$ represents the penalty functions.

This method transforms a constrained optimization problem into that of an unconstrained one. The individual weightings $w_{m}$ help normalize the penalty terms to ensure that violations are not under- or overrepresented. However, as noted by Price et al. (2005), the main drawback of the penalty approach is that the individual weightings must be correctly chosen to avoid the population from prematurely converging to infeasible or sub-optimal solutions. Under-penalizing slows down algorithm convergence while over-penalizing does the converse (yet at a greater risk of reaching a non-optimal solution).

Classical penalty functions are typically subdivided into two categories, namely: 1) interior functions and 2) exterior functions. An example illustrating the effect of the two functions on the modified objective function in Equation 2.7 is given in Figure 2.11 (with the global optimum denoted by point $A$ ). 


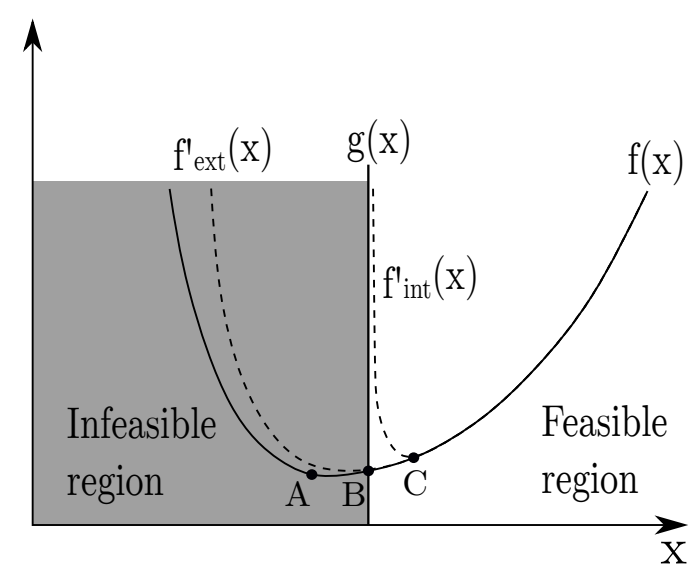

Figure 2.11: Interior and exterior penalty constraint handling

Interior penalty functions have an increasing effect as vectors tend towards infeasible solutions but have a small effect when far away from constraint boundaries. One of the biggest drawbacks of these functions is that they require a trial vector to lie within the feasible domain and as such do not work when a constraint is violated (Price et al., 2005). This not only requires re-initialization of infeasible trial vectors to feasible ones, but can also lead to sub-optimal solutions for near bound solutions (as indicated by point $C$ in Figure 2.11). Typical formulations of interior functions include barrier functions

$$
p_{m}(\mathbf{x})=\frac{-1}{g_{m}(\mathbf{x})}
$$

or log barrier functions

$$
p_{m}(\mathbf{x})=-\ln \left(-g_{m}(\mathbf{x})\right)
$$

Exterior functions on the other hand are not restricted to only feasible vectors but can tolerate those located within non-feasible regions. However, the further a trial vector lies outside of the feasible domain, the greater the influence the function will have on the original objective function (as illustrated by $f_{\text {ext }}^{\prime}(x)$ in Figure 2.11). The effect of these functions is to steer non-feasible solutions towards feasible ones rather than to obstruct them with an insurmountable barrier (Price et al. 2005$)$. Common exterior penalty functions include:

$$
p_{m}(\mathbf{x})= \begin{cases}g_{m}^{2}(\mathbf{x}) & \text { for } g_{m}(\mathbf{x})>0 \\ 0 & \text { otherwise }\end{cases}
$$

Or 


$$
p_{m}(\mathbf{x})= \begin{cases}\cosh \left(g_{m}(\mathbf{x})\right)-1 & \text { for } g_{m}(\mathbf{x})>0 \\ 0 & \text { otherwise }\end{cases}
$$

\section{Direct constraint handling}

One method that does not run the risks associated with that of the previous weighted penalty sum approach is that of the simpler direct constraint handling method. In early versions of this method (Kjellstrom and Taxen, 1981), constraints are initially relaxed until all vectors in the population meet such constraints. These constraints are then systematically tightened to motivate trial vectors towards feasible solutions. More recently, Lampinen (2002) takes this one step further by driving vector solutions in the direction where constraint violations decrease when found to be infeasible. This is illustrated in Figure 2.12 with the infeasible unconstrained vector at point $A$ being driven along the constraint function $g_{1}(x)$ towards the feasible constraint optimum at point $A^{\prime}$.

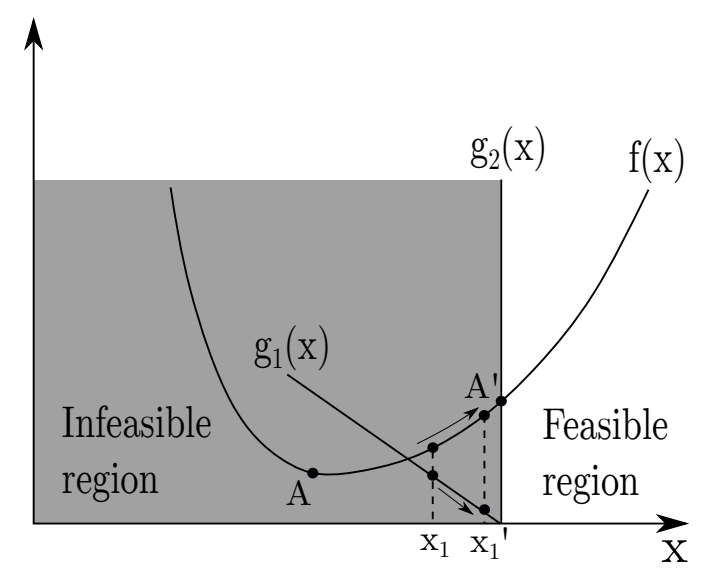

Figure 2.12: Direct constraint handling

\subsection{Concluding remarks}

Based on the current literature study, several concluding remarks are made and discussed.

The TLF is a known flow phenomenon within turbomachinery and has been the subject of many studies. The cause of the TLF over the blade tip is thought to be predominately pressure driven. Cumpsty and Storer (1991) propose that it is the pressure on the blade suction side that is thought to control the chord-wise 
distribution of the TLF.

The subsequent formation of the TLV (due to the momentum transfer from the TLF) has a detrimental effect on fan performance. The major losses associated with the formation of the TLV seem to be mixing related due to the shearing caused by the TLF and main flow interaction; this is described by Cumpsty (2004) in the form of a total loss coefficient.

Fan static pressure rise and fan static efficiency are found to increase across all flow rates with a reduction in tip clearance. However, the required tolerancing for such small tip clearances during manufacturing is not always feasible in large scale axial flow fans (both for practical and economic reasons). As such, larger than optimal tip clearances are usually implemented.

The blade tip edge is found to have a significant effect on the formation of the TLV. A sharp edge is favourable as it induces weaker secondary vortices which mix with the main flow thereby incurring fewer losses. A sharp edge also induces separation at the tip clearance entrance (tip edge on blade pressure side). This in turn causes a vena contracta which reduces the effective tip clearance thereby reducing the TLF.

The TLF is also influenced by the blade thickness. Denton (1993) introduces a ratio between the tip clearance and blade thickness where mixing within the tip gap would occur. It is this premature mixing of the TLF within the tip gap that is thought to incur fewer losses. However, Denton (1993) is of the opinion that this is not the case and that no difference will become apparent whether mixing takes place within the tip region or within the mainstream flow.

Various tip end-plate designs have been employed in an attempt to improve fan performance. Corsini et al. (2006) developed a constant thickness end-plate design which locally thickened the blade tip on the blade pressure side with respect to a reference radial dimension used in leakage vortex control. This design is further developed using an inverse swirl ratio (Rossby number) to define vortex breakdown. The findings of all subsequent designs indicate an improvement of fan total efficiency at lower than peak efficiency flow rates.

Three-dimensional numerical modelling is required in order to accurately capture the flow phenomena within the tip region. Various methods exist in literature whereby the domain upstream and downstream of the fan blade is modelled, with an extended annular duct configuration being most favoured by the majority of the publications studied in this thesis. 
Various open-source meshing alternatives are discussed, each having their own respective advantages and disadvantages over commercial software packages. Of the numerical models outlined, solving the RANS equations in steady state is considered an effective tool in vortical structure detection. Various turbulence models are discussed, each being better suited for specific applications. Two methods exist whereby the wall boundary layer is modelled, that being high- and lowReynolds approaches. Of the two, low-Reynolds modelling integrates through the wall boundary layer thus providing for a more accurate representation of the boundary layer development.

Various algorithms exist whereby the effective design optimization of turbomachinery is carried out. However, performance of these algorithms is situational dependent and as such more information is required before algorithm selection can be carried out. Nonetheless, two searching methods are selected for further investigation.

Based on the conducted literature study, a few concluding remarks with respect to the current understanding of TLF can be made, namely:

- There is uncertainty within the literature regarding the influence of each blade side pressure field (that being the high pressure or low pressure side of the blade) on controlling the TLF.

- Corsini and Sheard (2007a) only made use of one end-plate thickness based on a reference radial dimension to control the TLV. The effect of varying this thickness on the control of the TLV is unknown.

- The end-plate designs of Corsini and Sheard (2013) only have a beneficial operating range at lower than peak efficiency flow rates. A means by which the improvement of fan performance for higher than peak efficiency flow rates through the use of a tip appendage design is currently unavailable. 


\section{Chapter 3}

\section{Periodic three-dimensional numerical model}

The following sections outline the procedures used in meshing and simulating the periodic three-dimensional numerical model (P3DM) of the scale M-fan. As noted in Section 2.4.3 by Inoue and Furukawa (2002), the use of the RANS equations is considered an effective tool in vortical structure resolution. As such, both the mesh and simulation in the current study are set up for solving using the RANS equations as this significantly reduces computational effort when compared to other simulating methods.

\subsection{Computational domain}

Figure 3.1 shows the computational domain of the scale M-fan (details given in Appendix A) with its specified patch names and dimensions (as determined in the sensitivity analysis shown in Appendix B). The domain consists of a $1 / 8^{\text {th }}$ annular periodic section with an extended hub and casing to form the annular blade passage. Likewise with previous studies focusing on the tip region (see Section 2.3), an annular section is deemed sufficient for the intended purpose of this study. 


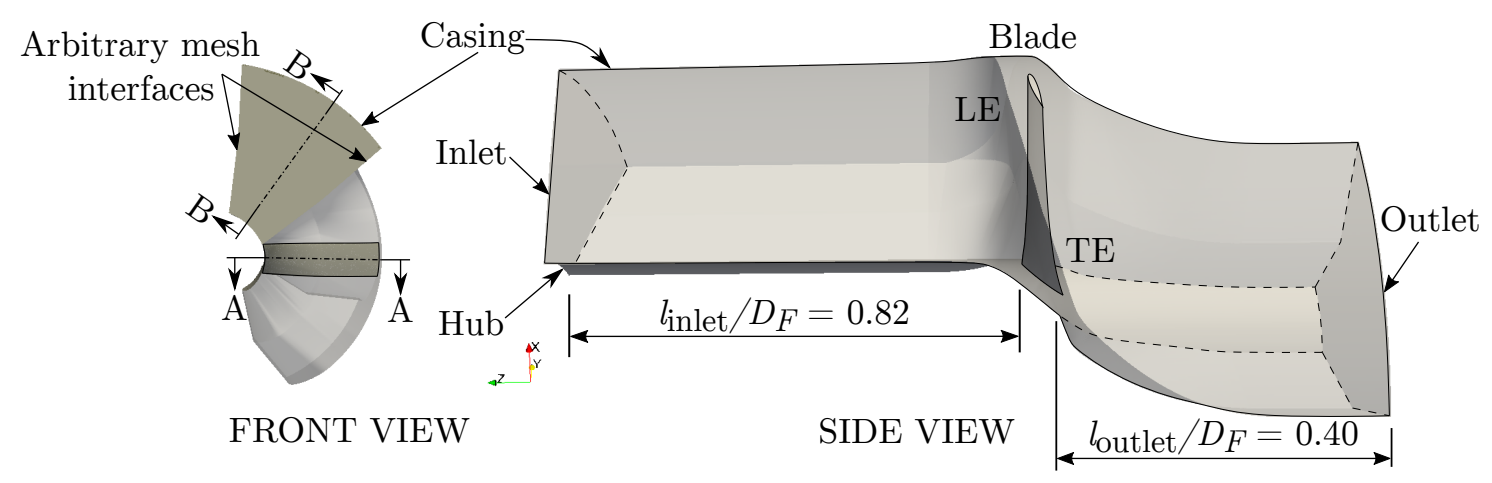

Figure 3.1: Periodic three-dimensional numerical model computational domain

The domain is created through the use of a CAD software package (Autodesk Inventor Professional 2018). This is done by importing the blade profile as a solid geometry and then creating the remainder of the domain around the blade. As can be seen in Figure 3.1, the domain is rotated about the blade's axis of rotation to align the domain boundaries immediately surrounding the blade to be parallel to the blade chord. This is in order to ensure a higher mesh quality as the distance between boundaries surrounding the blade are more uniform and therefore require less deformation during the meshing process.

Post domain creation, the geometry is exported in an initial graphics exchange specification (IGES) format. Thereafter, the open-source software Salome 8.5.0 is used to separate the boundary faces into patches. These patches are then combined and exported into a single STL formatted file which is then used in the mesh creation procedure. The STL file has to be watertight to ensure proper meshing, therefore a surface check is performed using OF to ensure the file meets this requirement.

\subsection{Meshing}

\subsubsection{Strategy}

From the literature review it can be seen that the various open-source meshing software packages have their own advantages and disadvantages. cfMesh is able to more easily produce a basic mesh with a higher capability of edge file editing. However, its limitations in total layer thickness growth, while maintaining enough refinement to capture geometrical features, leaves doubt as to the resultant mesh's ability to accurately capture the boundary layer development within the near-wall 
region.

SHM is limited in its base mesh creation in that it suffers when meshing complex geometries (for example fan blades with a high blade twist). The cells near patch surfaces are not always aligned orthogonal to the surface and therefore become highly distorted during the layer addition process. However, the layer addition process of SHM allows the user greater control in determining how the layers are grown, yet can cause unacceptable mesh quality if not carefully considered.

By considering each software's capabilities, it is possible to create a meshing strategy that involves using a combination of the two programs to take advantage of their respective strengths. As previously mentioned, the major advantage of this meshing strategy as opposed to commercial meshing programs is cost reduction. The proposed meshing strategy involves a three stage meshing procedure, namely: base mesh creation, preparation and layer addition. The meshing procedure is herewith discussed:

\section{Stage 1: Base mesh creation}

cfMesh is used to create the base mesh during the first meshing stage, as illustrated in Figure 3.2. This allows the user to define all the required areas for refinement while correcting for orthogonality near patch surfaces for later layer addition. The user should make the refinement as large as possible while still being able to capture geometrical features.

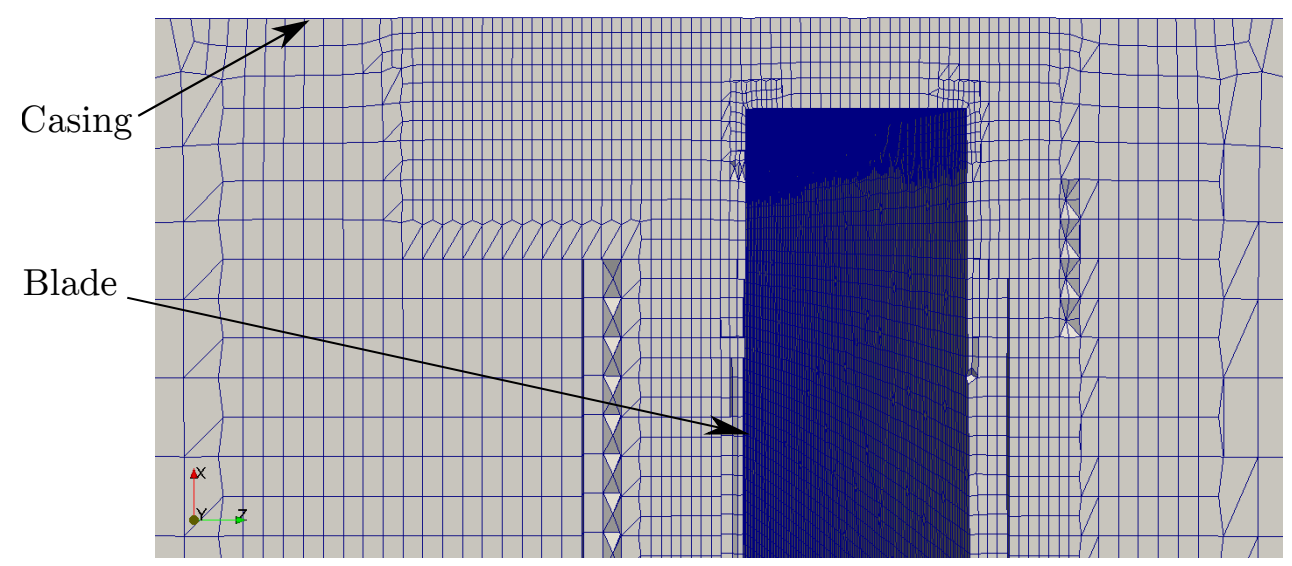

Figure 3.2: BaseMesh creation (section A-A Figure 3.1)

Edge refinement for the fan is divided into leading, trailing and tip edges. The trailing edge is split $10 \mathrm{~mm}$ above the hub surface to allow for a coarser refinement 
near the hub to ensure proper layer growth in the later stages (this would not be possible if using SHM). Thereafter, the mesh is checked to ensure mesh quality is not violated.

\section{Stage 2: BaseMesh preparation}

Shown in Figure 3.3, the built-in OF function refine WallLayer is used in preparing the base mesh for later layer addition. The cells' aligning patches that require layer addition are split multiple times using a set of predefined refinement factors. This is done to limit the maximum volume ratio between the added layer cells and the base mesh's internal cells to allow for a smooth transfer of information between cells. Care should be taken during this stage as the refinement function does not take mesh quality into account. This means that the mesh can easily gain an unacceptable mesh quality.

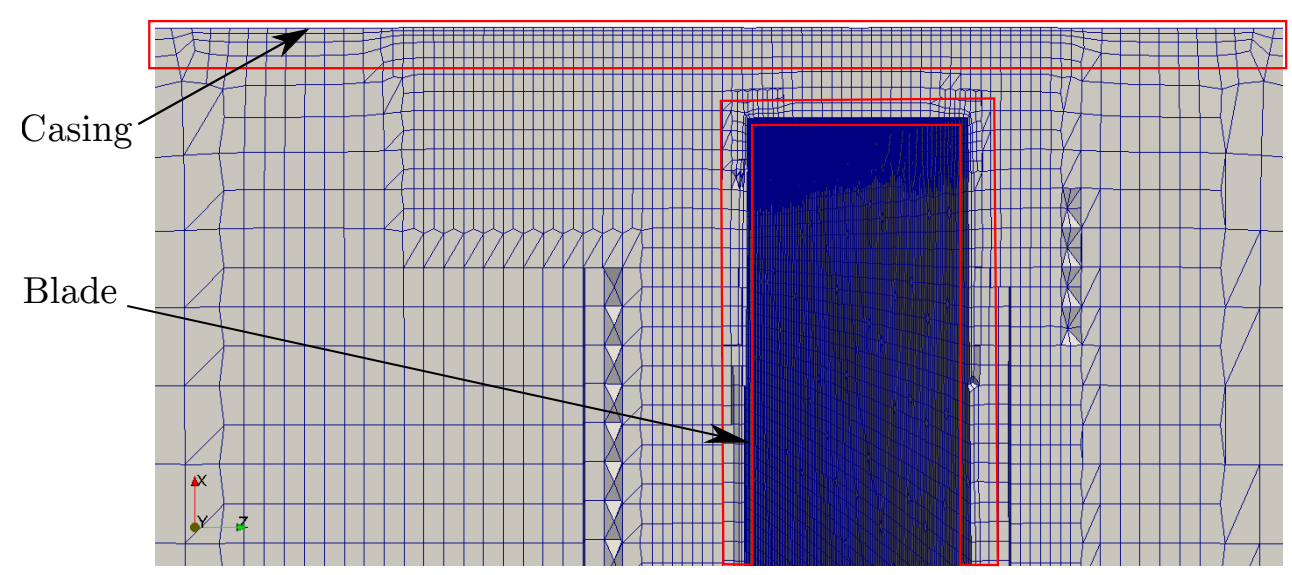

Figure 3.3: BaseMesh preparation (section A-A Figure 3.1)

\section{Stage 3: Layer addition}

The final stage makes use of SHM's layer addition phase. By switching off all mesh quality controls and carefully selecting the layer addition settings (to maximize growth) the layers are added to the specified patches. Although this method leads to complete layer growth, the final mesh quality is highly dependent on the chosen total layer thickness and local refinement level. These two meshing parameters are inversely proportional to acceptable mesh quality. If the total layer thickness is too large for a high level of refinement, the cells become warped and produce an unacceptable mesh quality. 
The hub layers are added first to limit the deformation of the layers around the blade to the final layer addition phase. The layer addition process is split up into two addition phases: outer layer addition and near-wall layer addition. The outer layers are desired to have a higher expansion ratio, usually around 1.2 to 1.25 , to limit the maximum volume ratio between the added cells and the internal mesh. The near-wall layers are desired to have a lower expansion ratio, usually 1.05, with the total added layers being in the range of 5 to 10 . The total number of added layers and expansion ratios are highly dependent on each other to satisfy mesh quality. By using the nMedialAxisIter setting in SHM, which limits the deformation of the cells away from the surface patch that the layer addition is applied to, the number of cells that become deformed can be controlled (to some degree) and therefore a better mesh quality is achievable. All these settings are adjusted to optimize the number of layers and overall mesh quality.

The blade and casing layers are added in a similar manner to the hub. The process can be seen by comparing Figure 3.3 to Figure 3.4. Since there is a tip gap between the blade tip and casing, these layers have to be added simultaneously to ensure that their respective growths do not interfere with each other. Noting this, it becomes apparent that the layers within the tip region have a tendency to become distorted as they grow in opposing directions (as shown in Figure 3.4 with the growth direction indicated with red arrows). The nMedialAxisIter setting is again used here to limit this deformation and produce an acceptable mesh quality.

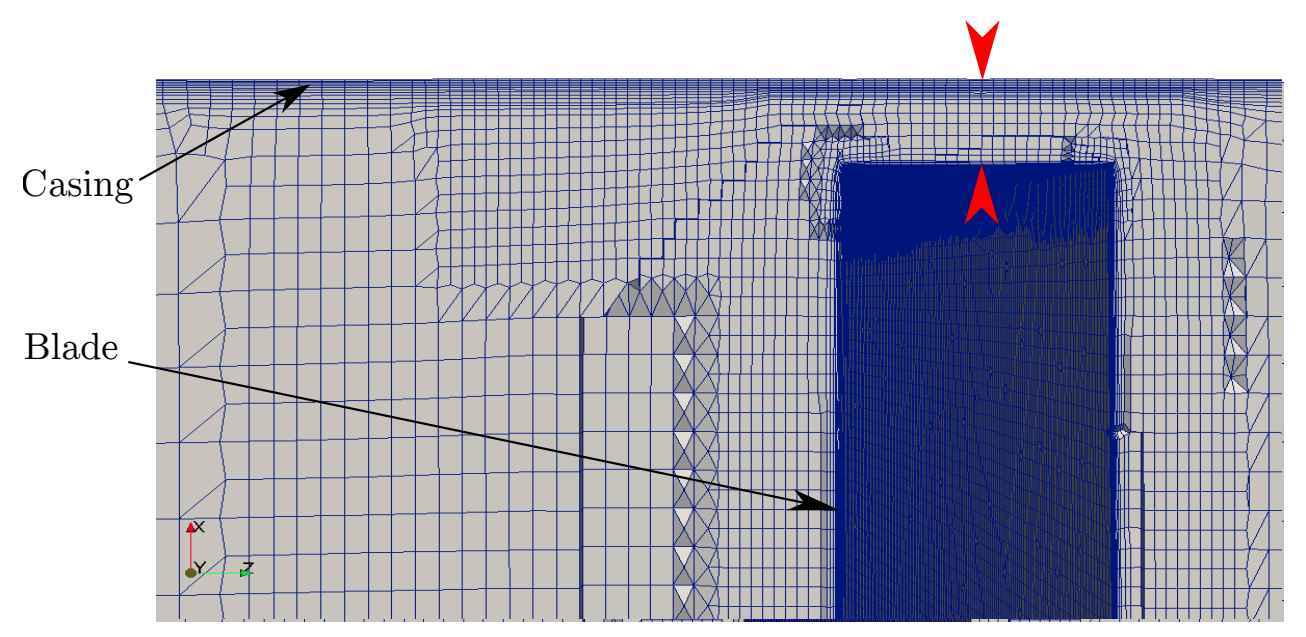

Figure 3.4: Layer addition (section A-A Figure 3.1 


\subsubsection{Construction}

Figure 3.5 shows the final computational mesh which is of a hybrid cell construction with predominately structured hexahedral cells. An exploded view of the rotor domain can be seen with surface projections of various locations around the blade included. This view is shown for illustrative purposes as the entire computational mesh is in fact whole in its construction.

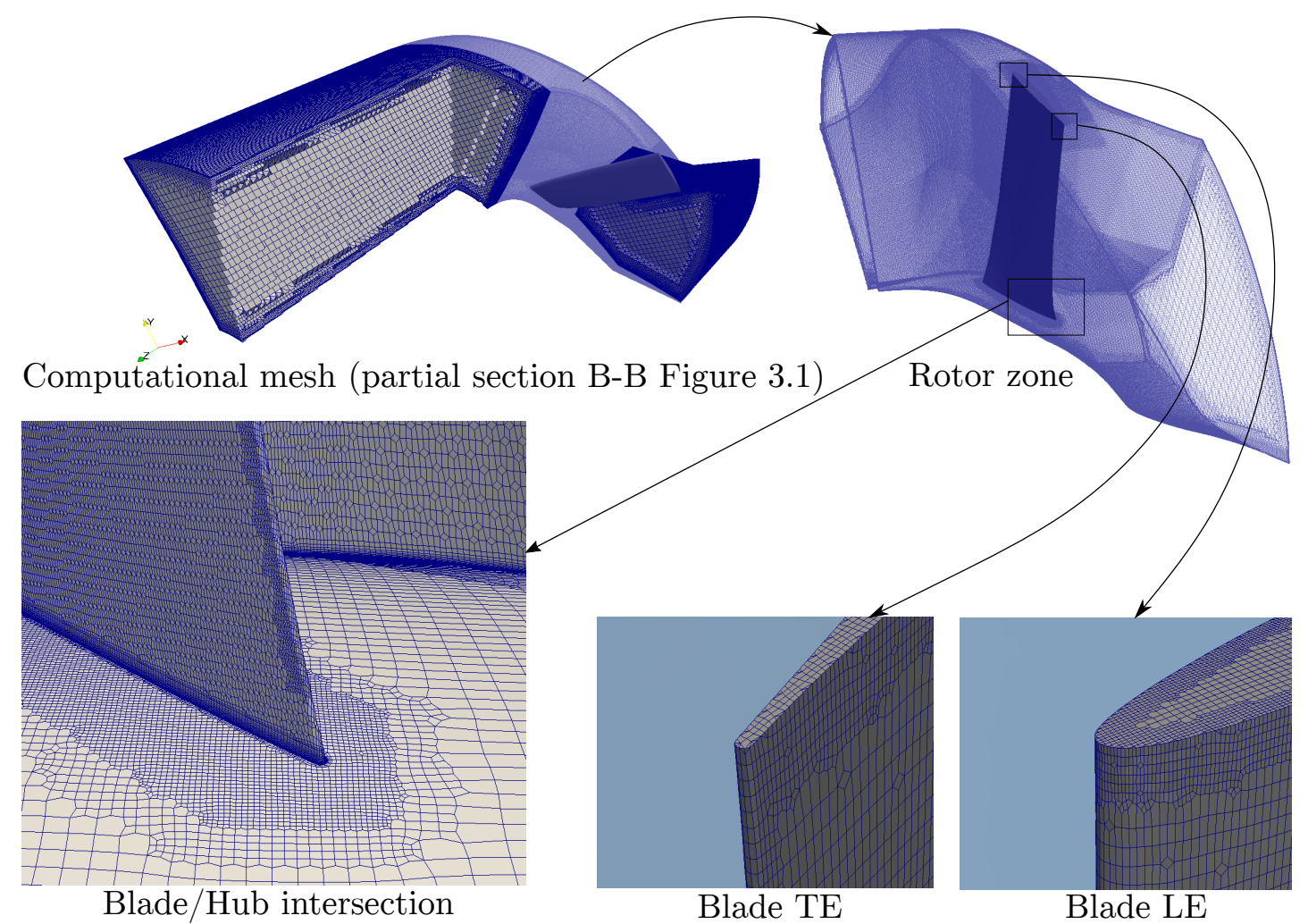

Figure 3.5: Periodic three-dimensional numerical model computational mesh

In order to accurately capture the effect of the boundary layer growth within the tip region, noted in Chapter 2.2.4 to have an effect on the TLF, the mesh is created based on a low-Reynolds number numerical approach. This means that the mesh is constructed such that the $y^{+}$number along the wall boundaries are in the range of $1<y^{+}<5$ in order to accommodate the viscous sublayer and therefore make use of enhanced wall functions.

The enhanced wall functions facilitate the development of the boundary layer within the near-wall region based on the laminar-to-turbulent $y+$ value derived 
from the $k$ and $\epsilon$ values (OpenCFD Ltd, 2018). The laminar-to-turbulent transition point is based on the intersection point between the viscous sublayer and buffer layer region within the boundary layer (which corresponds to a $y^{+}$value of 11). This means that when enabled, the functions operate in a low-Reynolds mode within the viscous sublayer and switch to a high-Reynolds mode thereafter. This significantly reduces the overall size of the computational mesh required as larger cell expansion ratios can be used for larger $y+$ values outside the viscous sublayer.

The refinement levels used are determined based on the mesh sensitivity analysis conducted in Appendix B. Table 3.1 shows the general guide used to represent acceptable mesh quality with the achieved mesh quality results from the mesh sensitivity analysis. The major quality aspects strictly adhered to are maximum skewness, maximum non-orthogonality and maximum aspect ratio. The sensitivity analysis shows that a mesh density of $10.8\left(10^{6}\right)$ cells with at least 27 elements within the tip region is sufficient for further numerical analysis. The final domain dimensions are shown in Figure 3.1 .

Table 3.1: Mesh quality control parameters

\begin{tabular}{lll}
\hline Quality control & Constraint & Achieved \\
\hline Max volume ratio & $<12$ & 11.9 \\
Maximum number of cells & $<12\left(10^{6}\right)$ & $11.8\left(10^{6}\right)$ \\
Max aspect ratio & $<100$ & 53.8 \\
Max skewness & $<4$ & 2.6 \\
Non-orthogonality, $\left[^{\circ}\right]$ & $<70, \max 80$ & $\max 71.4$ \\
\hline
\end{tabular}

\subsection{Boundary conditions}

The boundary conditions assigned to the specified patches, as labeled in Figure 3.1 . are as follows:

- Inlet*: A fixed, uniform flowRateInletVelocity profile is imposed on the inlet patch. This boundary condition applies a constant velocity vector based on the specified patch area and a user specified volume flow rate. A fixed, uniform turbulentIntensityKineticEnergyInlet and turbulentMixingLengthFrequencyInlet profile is applied for the turbulent kinetic energy and specific rate of dissipation, respectively. The estimation of which is discussed in Appendix C. A zeroGradient boundary condition is applied for the pressure field. 


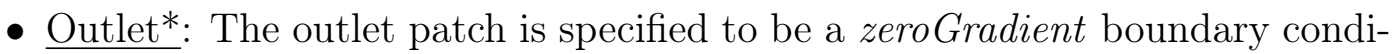
tion for all fields.

- Wall: All wall boundaries are specified with a no slip boundary condition for velocity. Enhanced wall functions are used for the turbulent kinetic energy, specific rate of dissipation and turbulent viscosity fields with the lowReynolds form enabled where applicable. All turbulence parameters are set to a uniform value of $1\left(10^{-10}\right)$ at the wall to accommodate the viscous sublayer. This value is chosen to be less than that of the simulation's specified writing precision. A zeroGradient boundary condition is applied for all pressure fields.

- cyclicAMI: Two cyclic arbitrary mesh interface (AMI) boundary conditions are specified on either side of the domain. These represent the coupled boundaries of the $1 / 8^{\text {th }}$ annular section. The AMI boundary condition accommodates mesh non-conformity by allowing each face to accept contributions from partially overlapping faces from the neighbouring patch, with the weights defining the contribution as a fraction of the intersecting areas (The OpenFOAM Foundation, 2014). This is to allow for the transfer of information by interpolating between the periodic patches. However, in order to limit any possible loss in accuracy caused by the mesh non-conformity, the boundaries are meshed with a higher refinement level (as shown in the sensitivity analysis conducted in Appendix B in order to limit the maxima and minima patch weightings.

* The nut (turbulent viscosity) field for the specified patches is set to calculated. This means that the turbulent viscosity is determined based on the turbulence parameter values.

\subsection{Numerical settings}

The following subsections outline the numerical settings used in simulating the scale M-fan. The settings used are focused on solution accuracy while maintaining solution stability. However, the settings are chosen such that mesh quality is taken into account, as this has the greatest influence on both solution accuracy and stability.

\subsubsection{Solver settings}

Table 3.2 shows the solution solver settings used. The gradient schemes are chosen to be second order accurate using Gauss linear. Although less accurate than that 
of the leastSquares method, this method is chosen as it provides a less oscillatory solution on tetrahedral meshes (Wolf Dynamics, 2013), as is the case for the proposed meshing procedure at the interference regions between mesh refinements.

Table 3.2: Solver settings

\begin{tabular}{lll}
\hline Scheme & Field & Setting \\
\hline Gradient & default & Gauss linear \\
& pressure & cellLimited Gauss linear 1 \\
\hline Divergence & default & none \\
& velocity & bounded Gauss \\
& & linearUpwindV grad(U) \\
& turbulence & bounded Gauss upwind \\
& effective viscous stress & Gauss linear \\
& vorticity & Gauss linear \\
\hline Laplacian & default & Gauss linear limited 0.5 \\
\hline Surface normal gradient & default & limited 0.5 \\
\hline
\end{tabular}

As the pressure field is highly influenced by the velocity field, its gradient computations are cellLimited to be less diffusive which aids in solution stability at the mesh boundaries. This is to further aid the transfer of information between the boundary layer cells and internal cells as their cell sizes rapidly change at the interference regions.

A bounded Gauss linearUpwindV interpolation scheme is used to compute the convective terms of the velocity components. This scheme is second order accurate and applies a single limiter across all velocity components according to the direction of greatest change in gradient. This greater limits the velocity components which aids in solution stability. The bounded term adds a source term into the transport equation to aid in convergence; this term subsequently becomes zero at convergence. As the turbulent properties are most influenced by the velocity components, the interpolation schemes are chosen to be first order accurate to aid in convergence and solution stability.

The Laplacian schemes are required in computing the diffusion term in the momentum equations. The surface normal gradient schemes are used to evaluate the Laplacian schemes using Gaussian integration (OpenCFD Ltd, 2018). As the common mesh quality encountered using the current meshing procedure and geometry is upwards of 70 degrees maximum non-orthogonality, both the Laplacian 
and surface normal gradient schemes are limited to 50 percent weightings between solution accuracy and stability, as recommended by Wolf Dynamics $(2013)$.

\subsubsection{Solution settings}

Table 3.3 shows the solution controls used. The semi-implicit method for pressure linked equations (SIMPLE) is used to couple the pressure and velocity equations. The setting of the reference pressure cell is also shown in the table.

Table 3.3: Solution settings

\begin{tabular}{llll}
\hline Control parameter & Field/Algorithm & Setting & Value \\
\hline Solvers(Robust) & pressure, mass flux (Phi) & solver & GAMG \\
& & preconditioner & DIC \\
& & tolerance & $1\left(10^{-4}\right)$ \\
& & relTol & 0.1 \\
\hline Solvers(Accurate) & pressure, mass flux (Phi) & solver & PCG \\
& & preconditioner & DIC \\
& & tolerance & $1\left(10^{-7}\right)$ \\
& & relTol & $0.05 / 0.01$ \\
\hline Algorithm & SIMPLE & pRefCell & 0 \\
& & pRefValue & 0 \\
\hline Relaxation factor & pressure & - & 0.3 \\
& velocity & - & 0.4 \\
& turbulence & - & 0.4 \\
\hline
\end{tabular}

\subsubsection{Solution initialization and convergence}

The simulation is solved by rotating the mesh at an angular speed of $N_{m}=722 \mathrm{rpm}$ while fixing the hub and blade patches to a stationary reference frame. The simulation is initially run for 500 iterations while setting the outlet pressure field to a fixed value of zero. This is in order to initialize the flow field by constraining the outlet pressure field for increased stability. Thereafter, the outlet pressure field is set to a zeroGradient boundary condition while setting a reference pressure cell to a fixed value of zero in order to maintain boundedness of the solution. This is in order to not over constrain the solution at reduced outlet domain lengths.

The simulation is initially run using the robust solver settings (as shown in Table 3.3 with a geometric agglomerated algebraic multigrid solver (GAMG). This solver is initially used as it is suited for asymmetric matrices with efficient transport 
of information across the solution domain (The OpenFOAM Foundation, 2014). These settings help to speed up convergence as the solution is highly oscillatory in the initial stages of simulation; hence the use of the asymmetric matrix solver.

Figure 3.6 illustrates the solution's convergence criteria. Fan total-to-static pressure rise and fan power are used to monitor solution convergence. These values are chosen as they represent physical values by which fan performance is measured during experimental testing.

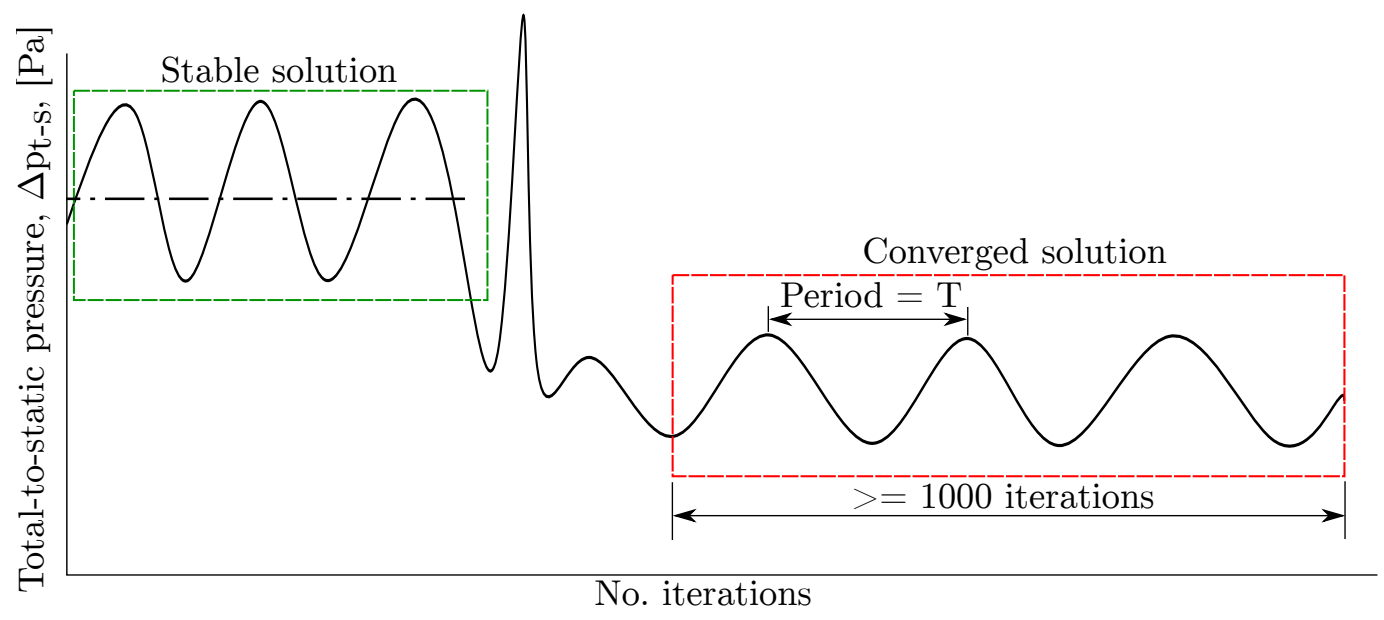

Figure 3.6: Solution convergence

Once the simulation has reached steady-state, deemed true once the monitored values consistently oscillate about a point, as shown on the left (green) in Figure 3.6, the tolerance and solver settings are updated. This procedure is repeated until the final settings represent that of the accurate solver settings shown in Table 3.3. The final convergence criteria (illustrated on the right (red) in Figure 3.6) is listed below.

- Change in fan total-to-static pressure rise $\left(\Delta p_{t-s}\right)$ is less than $0.5 \mathrm{~Pa}$ over at least 1000 iterations.

- Change in fan power is less than $5 \mathrm{~W}$ over at least 1000 iterations.

These values are chosen as no significant trend is observed when surpassing the iteration limit during the initial simulating of the scale M-fan. These values are lower than that of the scale M-fan's experimental test facility's maximum standard deviation error (presented in Chapter 4) and are therefore deemed acceptable. Once no noticeable trend is observed, the final solution is averaged over one period. 


\subsection{Turbulence modelling}

Section 2.4 .3 outlines various turbulence models used in literature which are specific to turbomachinery applications; all being better suited for specific flow conditions than others. Therefore, the work detailed in this section aims to conduct a comparative study of the various turbulence models in order to determine the most appropriate model to be used in the current study. Of all the models outlined in the literature study, three seem to stand out with regards to suitability for modelling an axial flow fan using a low-Reynolds number, enhanced wall function, RANS approach. The three turbulence models outlined are:

- Model 1: $k-\omega$ shear stress transport (SST) (linear 2 equation model)

- Model 2: Transitional $k-k l-\omega$ (linear transitional 3 equation model)

- Model 3: LaunderSharma $k-\epsilon$ (non-linear 2 equation model)

Table 3.4 compares the results obtained using the three turbulent models to a linear interpolated experimental value at the calculated design flow rate of Wilkinson et al. (2019):

Table 3.4: Turbulence model comparison of the scale M-fan at the $14.68 \mathrm{~m}^{3} / \mathrm{s}$ calculated design flow rate of Wilkinson et al. (2019) for a $6 \mathrm{~mm}$ tip clearance

\begin{tabular}{cccc}
\hline Turbulence model & $\boldsymbol{\Delta} \boldsymbol{p}_{\boldsymbol{t}-\boldsymbol{s}}[\mathrm{Pa}]$ & Power $[\mathrm{W}]$ & $\boldsymbol{\eta}_{\boldsymbol{t}-\boldsymbol{s}}[-]$ \\
\hline 1 & 74.70 & 2391.33 & 0.459 \\
2 & 84.58 & 2368.87 & 0.524 \\
3 & 72.89 & 2358.64 & 0.454 \\
Experimental & 73.99 & 2210.10 & 0.474 \\
\hline
\end{tabular}

Comparing the results obtained in Table 3.4 suggests that the $k-\omega$ SST model of Menter et al. (2003) is the most accurate in predicting the scale M-fan's performance with a maximum error of 1.0 percent on total-to-static pressure rise and 1.5 percentage points on total-to-static efficiency. The transitional $k-k l-\omega$ of Walters and Cokljat (2008) predicts the drag over the blade better; however, it over predicts the total-to-static pressure rise by up to 14.3 percent, thereby giving an optimistic value for the total-to-static efficiency. Of the three models, the nonlinear $k-\epsilon$ model of Launder and Spalding (1974) predicts blade drag best; yet, it under predicts on total-to-static pressure rise by up to 1.5 percent, therefore under predicting the fan's total-to-static efficiency. Based on these results, the $k-\omega \mathrm{SST}$ model of Menter et al. (2003) is chosen for further numerical analysis. 
It should be noted that selection of the current turbulence model is based on the idealized case of an annular pipe section. The numerical model assumes a uniform inlet velocity profile through consideration of the inlet boundary's displacement relative to the rotor section. During experimental testing, using the ISO test rig set-up, the bellmouth entry and front hub surface result in a flow profile that differs to that of a uniform velocity profile (either due to accelerated flow conditions or flow separation along the leading edge of the hub surface). This means that the current turbulence model's selection is only technically valid for the idealized case. 


\section{Chapter 4}

\section{Periodic three-dimensional numerical model validation}

In order to validate the suggested meshing strategy and P3DM model used in the current study, a set of reference performance characteristics need to be defined first. These characteristics then need to be used in order to compare and validate the numerical results against the experimental data. The following sections outline the validation process.

\subsection{Definition of fan performance characteristics}

Fan performance is quantified using three characteristic variables as a function of volumetric flow rate $(\dot{V})$. These are: fan total-to-static pressure rise $\left(\Delta p_{t-s}\right)$, fan power consumption $(P)$ and fan total-to-static efficiency $\left(\eta_{t-s}\right)$. The calculation of these variables, as defined in International Organization for Standardization (2007), are as follows:

Fan total-to-static pressure rise:

$$
\Delta p_{t-s}=p_{2}-p_{01}
$$

Fan power:

$$
P=T_{\text {shaft }} \omega_{\text {rotor }}
$$

Fan total-to-static efficiency:

$$
\eta_{t-s}=\frac{\dot{V} \Delta p_{t-s}}{P}
$$

where $p_{2}$ and $p_{01}$ represent the outlet and inlet static and stagnation pressure, respectively. 


\subsection{Aerodynamic performance comparison}

Figures 4.1 through 4.3 compare the performance characteristics of the experimental data (obtained from Wilkinson et al., 2019) against the numerical results (obtained in this study) of the scale M-fan at various tip clearances. A minimum of three operating points are simulated at each tip clearance, that being one point lower than design point flow rate (DP-), design point flow rate (DP), and one point higher than design point flow rate $(\mathrm{DP}+)$.

It should be noted that this comparison is only valid over the evaluated range of volumetric flow rates (that being $\mathrm{DP}_{-}, \mathrm{DP}$, and $\mathrm{DP}+$ ). This range is deemed acceptable as the volumetric flow rates commonly encountered during the fan's designed operation are predicted to fall within this region.

High oscillatory behaviour of the solution was found for all lower than design point flow rates. Consequently, a steady-state solution could not be obtained. As such, a large time-step transient solver (pimpleFoam) is used to simulate all lower than design point flow rates as the implicit solver provides for an unconditionally stable solution. The large time-step solver allows simulations with higher than normal Courant numbers $(\mathrm{Co})$, up to a value of 5 while maintaining solution accuracy (Wolf Dynamics, 2013).

Using this type of solver results in a decrease in computational time, yet it has a higher probability of introducing instability and temporal diffusion. Therefore, a $C o=5$ number is used as a maximum to initially speed up simulation convergence, after which (and depending on simulation stability) it is reduced. All simulations were run in parallel over 24 Intel ${ }^{\circledR}$ Xeon ${ }^{\circledR}$ CPU cores. Typical simulation times ranged from a maximum of 384 hours for the time-dependent solutions $\left(\dot{V}=9.29 \mathrm{~m}^{3} / \mathrm{s}, \bar{\Delta} \mathrm{t}=3.3 \times 10^{-6}\right.$ seconds $)$ to an average of 48 hours for the steady-state solutions.

The RMSE of each tip clearance's set of results is calculated in order to compare against the experimental data. As the total-to-static pressure rise at DP+ is relatively low in comparison to the other operating points, a magnification of the RMSE could result, thus providing for a skewed interpretation of results. Therefore, a flow rate half way between the higher two flow rates on the experimental performance curve is chosen as the $\mathrm{DP}+$ point and compared to a linear interpolation of the experimental data. 
CHAPTER 4. PERIODIC THREE-DIMENSIONAL NUMERICAL MODEL VALIDATION

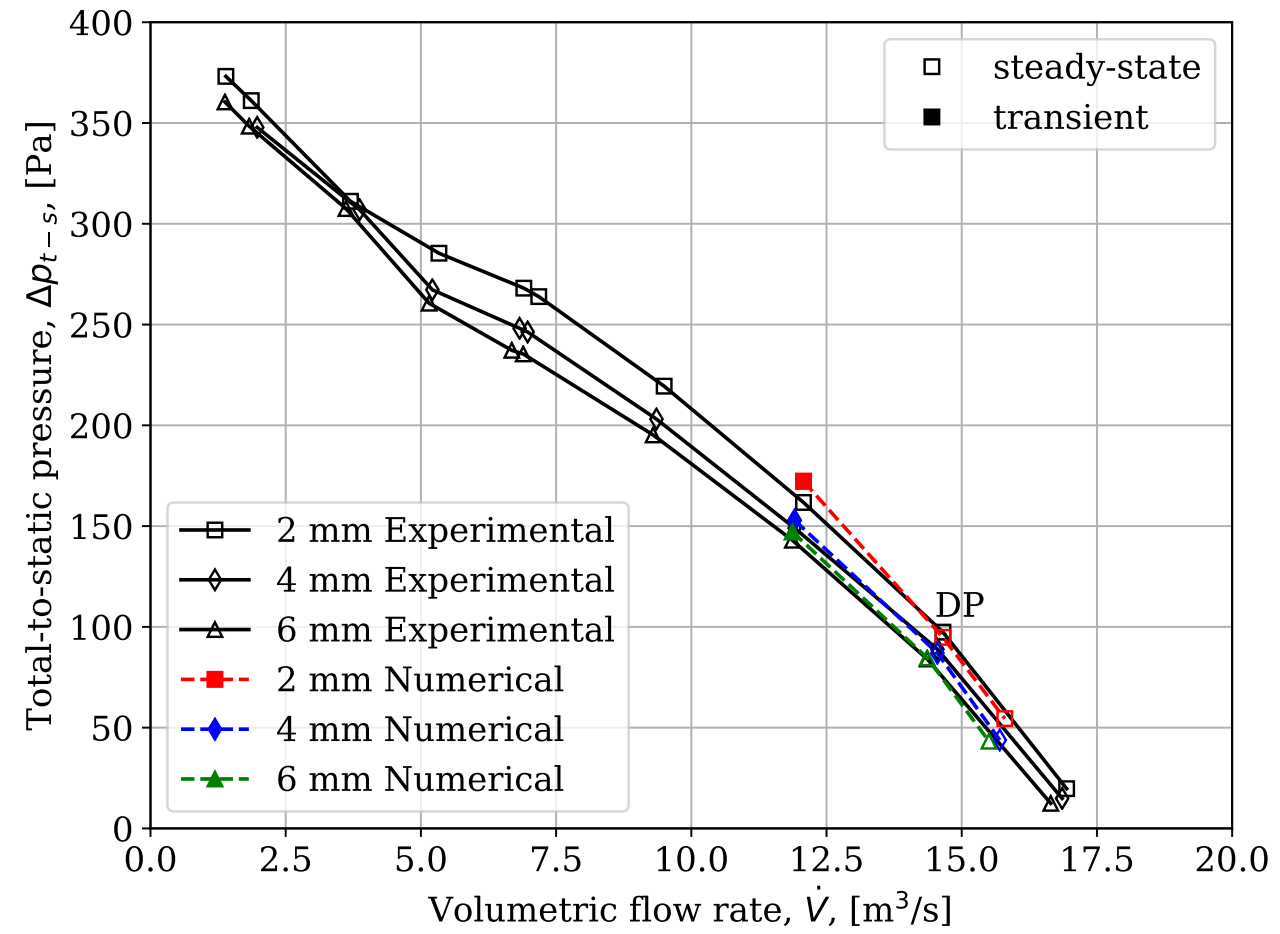

Figure 4.1: Scale M-fan experimental and numerical total-to-static pressure rise comparison at various tip clearances

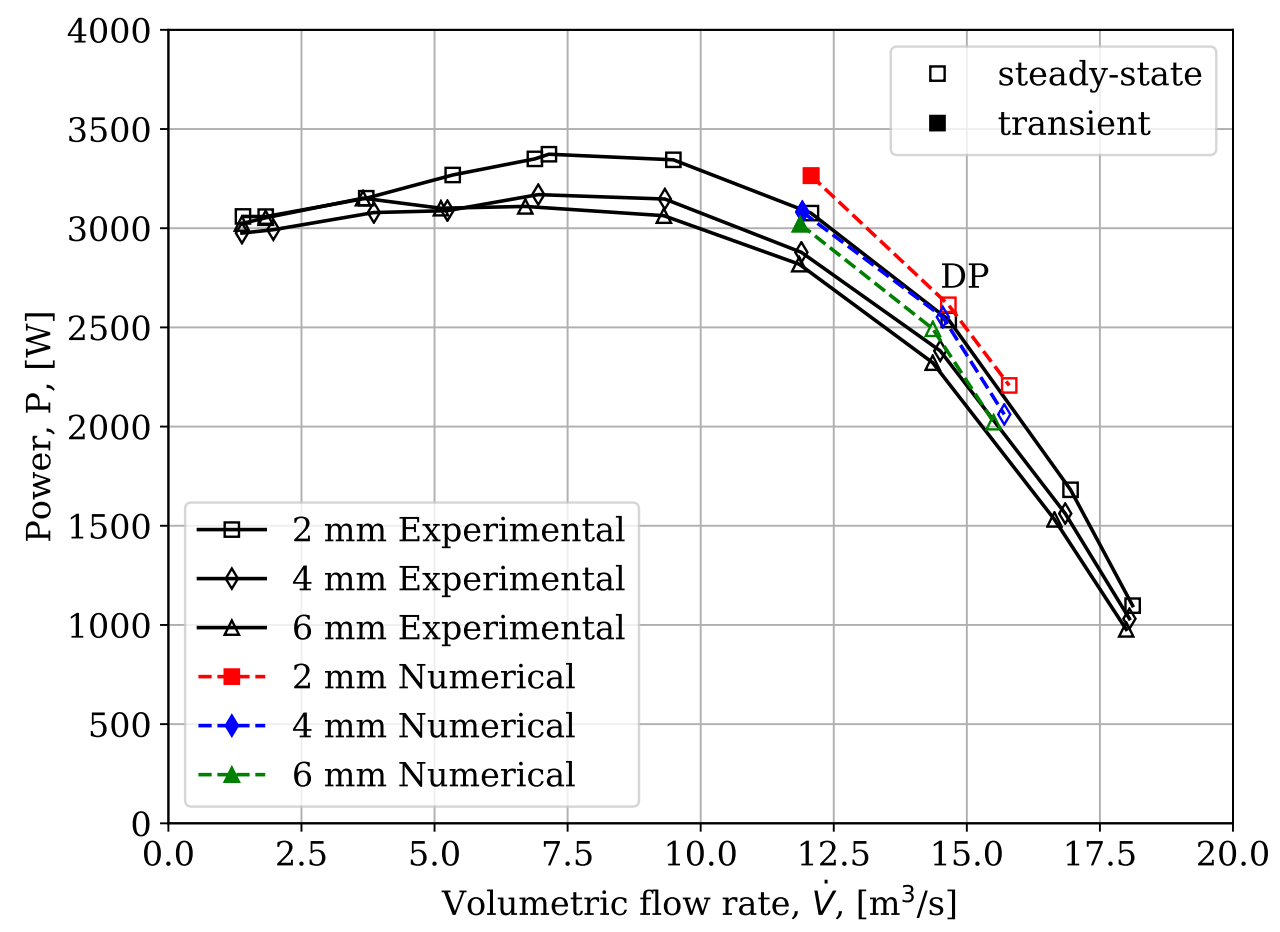

Figure 4.2: Scale M-fan experimental and numerical power comparison at various tip clearances 
CHAPTER 4. PERIODIC THREE-DIMENSIONAL NUMERICAL MODEL VALIDATION

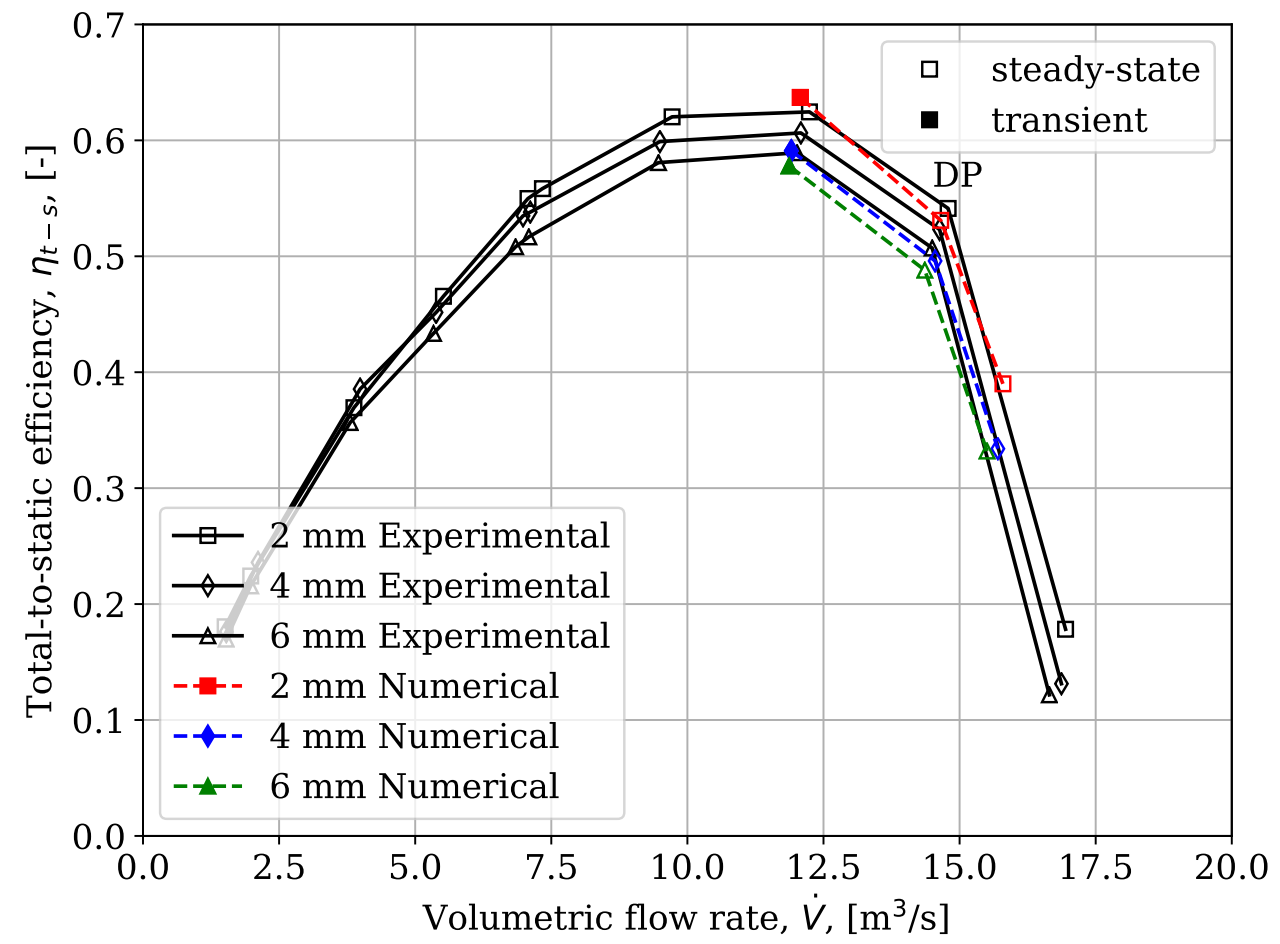

Figure 4.3: Scale M-fan experimental and numerical total-to-static efficiency comparison at various tip clearances

\subsection{Validation of results}

Validation is performed by assessing the numerical results in terms of its variation and bias against the experimental data.

\subsubsection{Variation}

Due to the experimental data likely consisting of error and uncertainty, the RMSE of the numerical results (relative to the experimental data) are compared to the maximum standard deviation of the experimental test facility. This is in order to determine an acceptable variation in the numerical results across the evaluated range of volumetric flow rates. Table 4.1 compares the RMSE of the numerical results to the maximum standard deviation of the experimental test facility (as used by Wilkinson et al., 2019). 
CHAPTER 4. PERIODIC THREE-DIMENSIONAL NUMERICAL MODEL VALIDATION

Table 4.1: Comparison of numerical root-mean-square error and maximum experimental test facility standard deviation of the scale M-fan at various tip clearances

\begin{tabular}{cccc}
\hline & \multicolumn{3}{c}{$\boldsymbol{R} \boldsymbol{M} \boldsymbol{S E}$} \\
Tip clearance & $\boldsymbol{\Delta}_{\boldsymbol{p}_{\boldsymbol{t}-\boldsymbol{s}}[\mathrm{Pa}]}$ & $\mathbf{P}[\mathrm{W}]$ & $\boldsymbol{\eta}_{\boldsymbol{t}-\boldsymbol{s}}[-]$ \\
\hline $2 \mathrm{~mm}$ & 6.678 & 220.31 & 0.0142 \\
$4 \mathrm{~mm}$ & 5.255 & 171.22 & 0.0194 \\
$6 \mathrm{~mm}$ & 3.772 & 129.61 & 0.0152 \\
Experimental $\sigma_{\max }$ & 5.221 & 28.45 & 0.0385 \\
\hline
\end{tabular}

Comparing the $\Delta p_{t-s} R M S E$ values in Table 4.1, it can be seen that only the $6 \mathrm{~mm}$ numerical result lies within the experimental test facility's maximum standard deviation. This could be due to the mesh sensitivity analysis (Appendix B) being conducted using a $6 \mathrm{~mm}$ tip clearance. This would result in fewer cells spanning the height of the tip gap for the $4 \mathrm{~mm}$ and $2 \mathrm{~mm}$ tip clearance cases thereby providing less resolution within this region. It would have been more correct to use the smaller $2 \mathrm{~mm}$ tip clearance, yet this would have meant an overall increase in the mesh cell count for all subsequent meshes and thus a further increase in computational time. However, as the current design is intended to improve the M-fan's performance at the design tip clearance (equivalent scale being $6 \mathrm{~mm}$ ), the smaller tip clearances are only used for comparative purposes and the results are therefore deemed sufficient.

Considering the $\eta_{t-s} R M S E$, all numerical results obtained fall within the experimental test facility's maximum standard deviation. This shows a strong correlation between the relative coupling of the numerical pressure and power.

\subsubsection{Bias}

In order to determine the bias of the numerical results, a comparison is made between the differences in numerical and experimental results to that found in literature. Table 4.2 compares the numerical and experimental result differences of the scale M-fan to various fans found in literature, evaluated at their respective design point flow rates. 
CHAPTER 4. PERIODIC THREE-DIMENSIONAL NUMERICAL MODEL VALIDATION

Table 4.2: Difference in numerical and experimental results of the scale M-fan compared to literature evaluated at each fan's respective design point flow rate

\begin{tabular}{|c|c|c|c|}
\hline \multirow[b]{2}{*}{ Fan } & \multirow[b]{2}{*}{ Tip clearance } & \multicolumn{2}{|c|}{ Variable } \\
\hline & & $\Delta p_{n / e}[\mathrm{~Pa}]$ & $\Delta \eta_{n / e}[-]$ \\
\hline Scale M-fan (this study) & $4.0 \mathrm{~mm}$ & 0.57 & 0.024 \\
\hline Scale M-fan (Wilkinson et al., 2019) & $4.0 \mathrm{~mm}$ & 3.77 & 0.012 \\
\hline AC90/6 (Corsini and Sheard, 2007a) & $3.5 \mathrm{~mm}$ & 1.50 & 0.020 \\
\hline
\end{tabular}

The differences in numerical and experimental results achieved in this study are comparable to that obtained by Corsini and Sheard (2007a) and Wilkinson et al. (2019) who made use of open-source and commercial software packages, OF and ANSYS FLUENT, respectively. This suggests that the proposed open-source meshing strategy may be appropriate in modelling the scale M-fan. Therefore, this provides for sufficient supporting evidence to further continue the use of the proposed meshing strategy in the design procedure. 


\section{Chapter 5}

\section{Effect of tip clearance and tip leakage vortex development}

A study into tip clearance effects on fan performance and the formation of the TLV needs to be conducted in order to gain insight into local flow phenomena present within the tip region and the effects thereof. This chapter aims to conduct a numerical investigation of such effects on the scale M-fan in four sections, namely:

1. Scaling of the M-fan with varying tip clearances.

2. Effect of tip clearance on fan performance.

3. Visualization of the TLV within the tip region.

4. Quantification of the TLV within the tip region.

\subsection{Fan scaling at various tip clearances}

As discussed in Appendix A, the scaling factor used to scale the M-fan does not remain constant when varying the tip clearance during experimental testing. This is due to the blade tip diameter being altered to achieve a desired tip clearance relative to a fixed casing diameter. As such, it would make sense to likewise adjust the design point flow rate when varying the tip clearance to compensate for this change in diameter. The various design point flow rates (as determined in Appendix A for the scale M-fan at various tip clearances are given in Table 5.1 . 
CHAPTER 5. EFFECT OF TIP CLEARANCE AND TIP LEAKAGE VORTEX DEVELOPMENT

Table 5.1: Scale M-fan specifications for various tip clearances $\left[N_{m}=722 \mathrm{rpm}\right]$

\begin{tabular}{ccc}
\hline Tip clearance & Diameter $[\mathrm{m}]$ & Flow rate $\left[\mathrm{m}^{3} / \mathrm{s}\right]$ \\
\hline $2 \mathrm{~mm}$ & 1.538 & 14.80 \\
$4 \mathrm{~mm}$ & 1.534 & 14.68 \\
$6 \mathrm{~mm}$ & 1.530 & 14.57 \\
\hline
\end{tabular}

In order to illustrate this effect further, comparison is made between using a constant design point flow rate (as done by Wilkinson et al. (2019) using the 14.57 $\mathrm{m}^{3} / \mathrm{s}$ design point flow rate of the geometrically similar $6 \mathrm{~mm}$ tip clearance case) as opposed to the current method of adjusting the flow rate when altering the blade tip diameter. The experimental results of the $4 \mathrm{~mm}$ tip clearance case using the two aforementioned methods are given in Table 5.2 , with the geometrically similar $6 \mathrm{~mm}$ tip clearance case included for comparison.

Table 5.2: Effect of scaling design point flow rate on scale M-fan experimental results (Wilkinson et al., 2019)

\begin{tabular}{ccccc}
\hline & & \multicolumn{3}{c}{ Variable } \\
Tip clearance & $\dot{\boldsymbol{V}}\left[\mathrm{m}^{\mathbf{3}} / \mathrm{s}\right]$ & $\boldsymbol{\Delta} \boldsymbol{p}_{\boldsymbol{t}-\boldsymbol{s}}[\mathrm{Pa}]$ & Power [W] & $\boldsymbol{\eta}_{\boldsymbol{t}-\boldsymbol{s}}[-]$ \\
\hline $4 \mathrm{~mm}$ & 14.68 & 84.75 & 2318.92 & 0.515 \\
$4 \mathrm{~mm}$ & 14.57 & 88.28 & 2357.33 & 0.525 \\
$6 \mathrm{~mm}$ (geometric) & 14.57 & 77.58 & 2249.67 & 0.494 \\
\hline
\end{tabular}

As evident, superior performance is observed when using the lower design point flow rate of the geometrically similar $6 \mathrm{~mm}$ tip clearance case $\left(14.57 \mathrm{~m}^{3} / \mathrm{s}\right)$ to evaluate the performance of the $4 \mathrm{~mm}$ tip clearance case. This becomes obvious when considering the location of the design point relative to the fan's operational curve. However, as the $4 \mathrm{~mm}$ tip clearance case effectively represents a larger diameter fan (due to the alteration of the blade tip diameter), it has an inherent advantage over the $6 \mathrm{~mm}$ tip clearance case as the two are evaluated at the same operating condition.

When considering the higher $14.68 \mathrm{~m}^{3} / \mathrm{s}$ design point flow rate (adjusted using the fan scaling laws), it too represents an increase in all fan performance metrics over the geometrically similar $6 \mathrm{~mm}$ tip clearance case; however, at reduced improvement margins. This represents a more conservative comparison between the two tip clearance cases as the respective design point operating conditions are altered to compensate for the change in fan diameter. As such, the more conservative approach is selected for use in the current investigation with the fan specifications 
in Table 5.1 being used for the remainder of this thesis.

It should be noted that alteration of the blade geometry to suit a particular casing diameter for various tip clearances means that the designs are no longer geometrically similar (although these differences are small). Changing the rotor diameter results in an alteration of the hub-to-casing diameter ratio. This means that the main axial velocity (which should be kept constant to maintain constant velocity triangles) is altered.

On the other hand, if you change the hub size to suit a particular tip clearance, you are changing the radial position of each aerofoil section; once again, you are modifying the velocity triangles/blade design matching. The correct way to maintain geometric similarity would be to alter the casing diameter to suit a desired tip clearance and keep all other variables constant. However, the practical aspects associated with implementing such a method means that it is not currently a viable option. As this is a comparative analysis in relation to the geometrically similar datum design, the differences between designs due to geometric dissimilarity will be assumed small.

\subsection{Aerodynamic performance analysis}

Figures 4.1 through 4.3 give an indication of the general trend in fan performance characteristics as the tip clearance is reduced. Comparing the results between the various tip clearances, an increase in fan performance across all flow rates becomes apparent with a reduction in tip clearance. These findings concur with that found by Ruden (1944); Venter and Kröger (1992).

Table 5.3 summarizes the numerical results obtained of the scale M-fan at various tip clearances at their respective design point flow rates. Numerical fan performance characteristics are found to improve by 15.6 percent in total-to-static pressure rise and 5.1 percentage points in total-to-static efficiency with a 66.6 percent reduction in tip clearance. The subsequent sections describe the investigation into this mechanism further. 
CHAPTER 5. EFFECT OF TIP CLEARANCE AND TIP LEAKAGE VORTEX DEVELOPMENT

Table 5.3: Numerical fan performance characteristics of the scale M-fan at various tip clearances evaluated at their respective design point flow rates

\begin{tabular}{ccccc}
\hline & & \multicolumn{3}{c}{ Variable } \\
Tip clearance & $\dot{\boldsymbol{V}}\left[\mathrm{m}^{\mathbf{3}} / \mathrm{s}\right]$ & $\boldsymbol{\Delta} \boldsymbol{p}_{\boldsymbol{t}-\boldsymbol{s}}[\mathrm{Pa}]$ & Power [W] & $\boldsymbol{\eta}_{\boldsymbol{t}-\boldsymbol{s}}[-]$ \\
\hline $2 \mathrm{~mm}$ & 14.80 & 89.81 & 2567.71 & 0.518 \\
$4 \mathrm{~mm}$ & 14.68 & 81.96 & 2503.39 & 0.481 \\
$6 \mathrm{~mm}$ & 14.57 & 77.72 & 2423.18 & 0.467 \\
\hline
\end{tabular}

\subsection{Vortex detection and visualization}

Normalized helicity $H_{\text {norm }}$ contours are used to visualize and detect the development of the vortex core near the blade tip. Normalized helicity is defined as:

$$
H_{\text {norm }}=\frac{U \cdot v}{|U||v|}
$$

where $U$ is the absolute velocity vector, $v$ is the absolute vorticity vector and $|U|$ and $|v|$ are their respective magnitudes. From Equation 5.1, it can be seen that this value correlates with the angle between the two vectors, ranging between -1 to 1. This indicates the orientation of the velocity vector with respect to the vorticity vector. This can then be used to determine the presence of a vortex core as both vectors tend to align themselves parallel within the core thereby tending to the outer limits of the given range (that being either -1 or 1 ).

Figure 5.1 shows the helicity contours of the scale M-fan at various tip clearances with a visualization of the TLV core. In all three cases, the helicity contours suggest the presence of a primary clockwise rotating vortex structure developing along the blade chord and on the blade suction side (denoted TLV/SS). One would expect the right-hand rotation to result in a positive helicity if streamwise oriented. However, this depends on how the vorticity sign is defined (which happens to be negative in this case). This is the reason for the apparent negative helicity value within the vortex core. All vortex cores originate between 0.2 and 0.4 chord fractions which agrees well with findings in literature (Corsini et al., 2010, Giuni, 2013, Ye et al. 2017). 


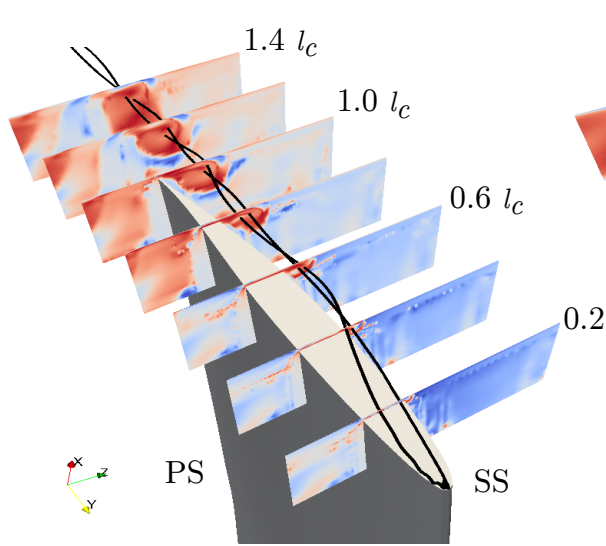

a)

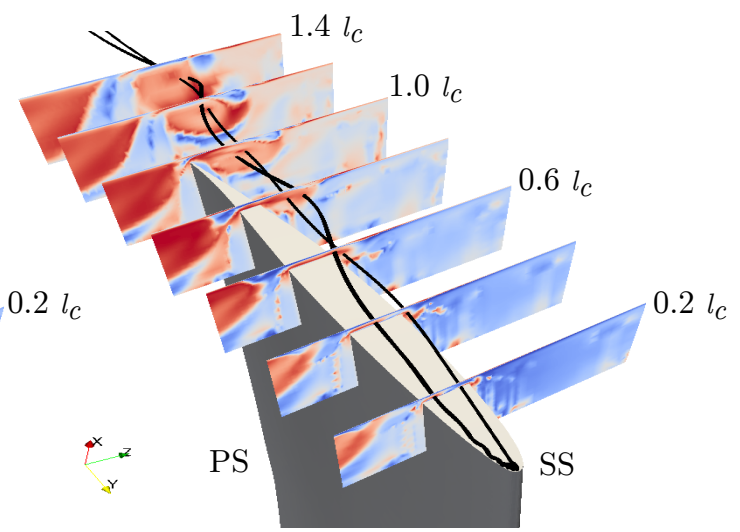

b)

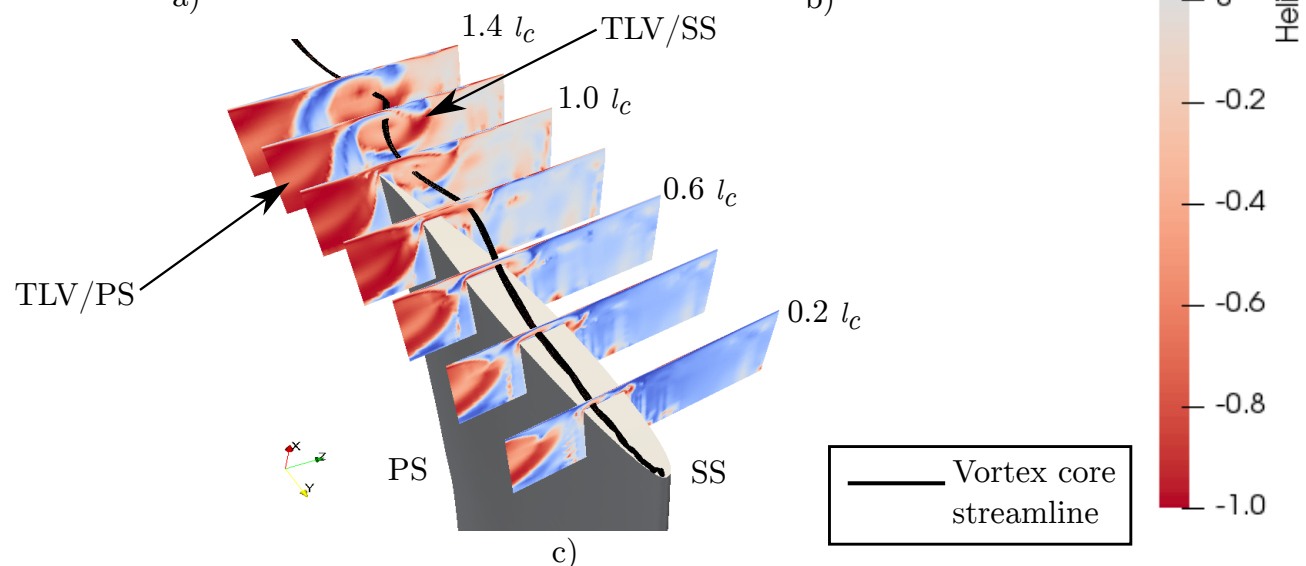

Figure 5.1: Normalized helicity $H_{\text {norm }}$ cross sectional contours of the scale M-fan at blade tip with suction side vortex (TLV/SS) visualization at various tip clearances evaluated at their respective design point flow rates: a) $2 \mathrm{~mm}$ tip clearance $[\dot{V}=$ $\left.14.80 \mathrm{~m}^{3} / \mathrm{s}\right]$, b) $4 \mathrm{~mm}$ tip clearance $\left[\dot{V}=14.68 \mathrm{~m}^{3} / \mathrm{s}\right]$, and c) $6 \mathrm{~mm}$ tip clearance $\left[\dot{V}=14.57 \mathrm{~m}^{3} / \mathrm{s}\right]$

Comparing the contours in Figure 5.1 through c, it is seen that an increase in tip clearance has the effect of increasing the size of the TLV/SS. Further away from the blade on the pressure side, it can be seen that a secondary vortex structure (identified by the dark red contours in the figure) is located closer to the blade surface and increases in size and intensity as the tip clearance is increased. These two vortex structures (of equal rotational direction) then interact along the blade chord and merge further downstream, forming a larger vortex structure.

Figure 5.2 visualizes the vortex development within the tip region over one blade pitch (blade rotation from left to right). As can be seen, the TLV/SS moves downstream and extends from the forward blade towards the path of the next 
blade, thereby becoming the secondary vortex structure (as previously identified in the helicity contours in Figure 5.1 and denoted TLV/PS). The merging of these two vortices affect the blade-to-blade flow field, as described by Corsini and Sheard (2013).

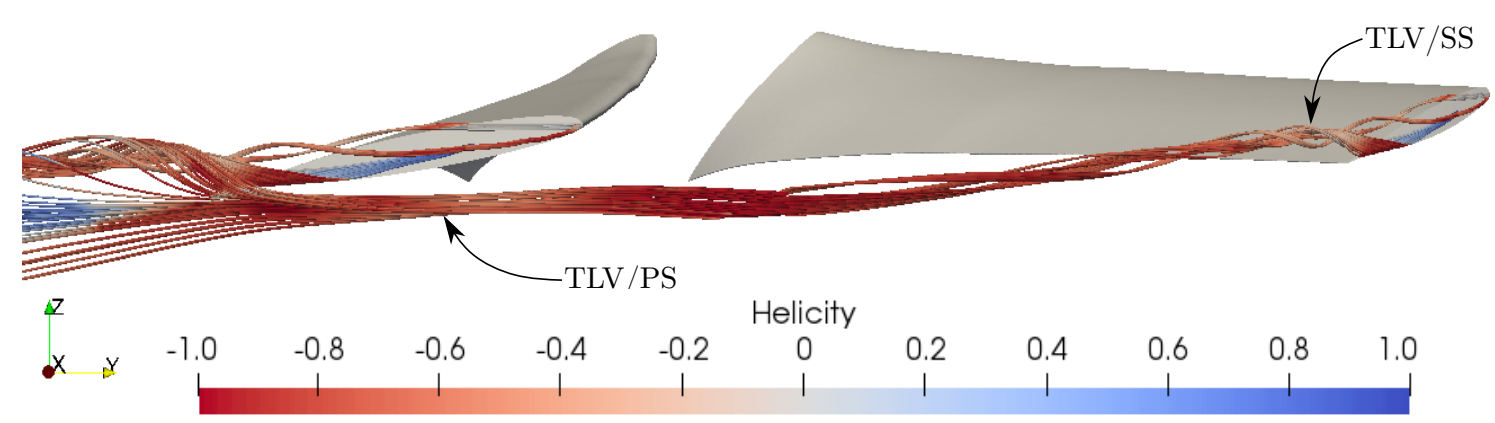

Figure 5.2: Blade-to-blade tip leakage vortex interaction of the scale M-fan with a $6 \mathrm{~mm}$ tip clearance at the fan's $14.57 \mathrm{~m}^{3} / \mathrm{s}$ design point flow rate

\subsection{Vortex quantification and analysis}

Figure 5.3 shows a comparison of the relative TLF velocity, $W_{T L F}$, and TLV/SS exit trajectory angle, $\beta_{T L V}$, along the blade chord for the scale M-fan at various tip clearances. The $\beta_{T L V}$ angle is measured relative to the blade chord for each chordwise abscissae. The determination of both vortex metrics is further discussed in Appendix D.1 and D.2, respectively.

Reducing the tip clearance has the effect of reducing the relative TLF velocity within the tip gap along the blade chord. This finding agrees with that found by Venter and Kröger (1992) who suggests that the reduction in the TLF velocity within the tip gap as a result of a reduction in the tip clearance causes a reduction in the associated pressure losses. 
CHAPTER 5. EFFECT OF TIP CLEARANCE AND TIP LEAKAGE VORTEX DEVELOPMENT

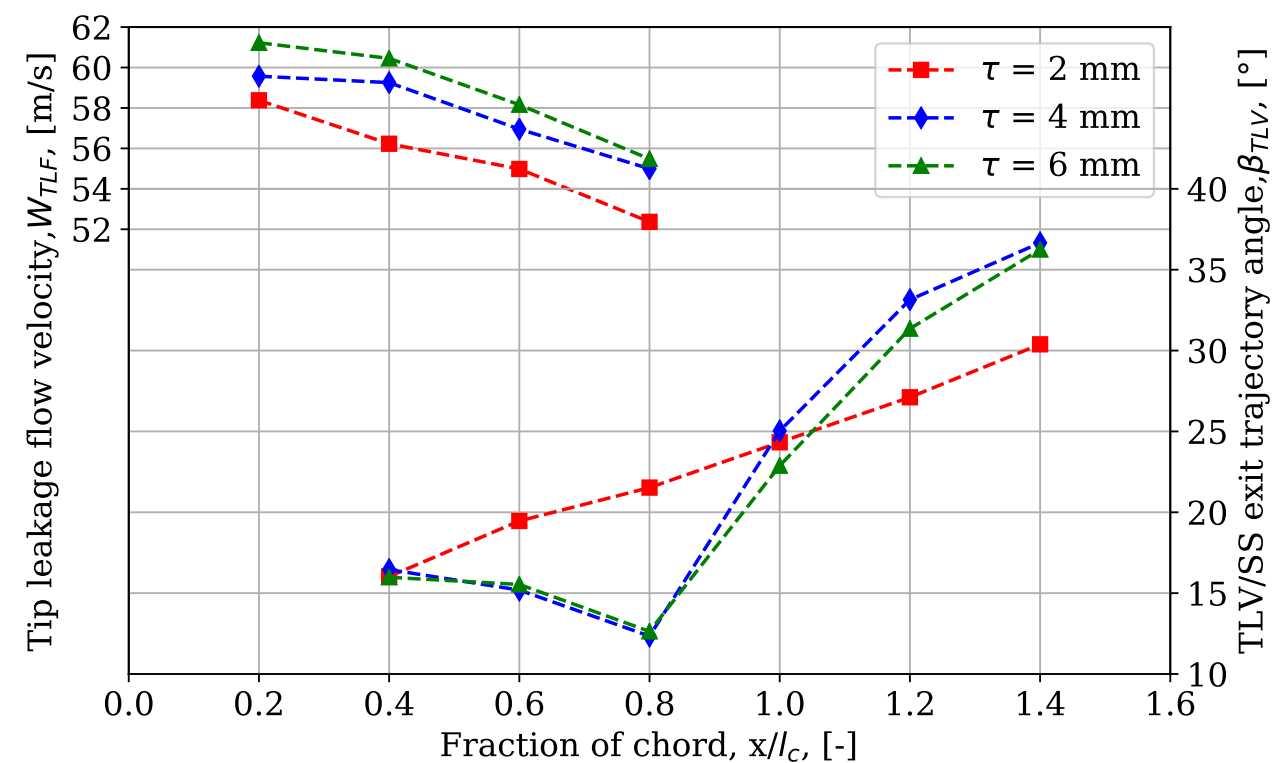

Figure 5.3: Relative tip leakage flow velocity $\left(W_{T L F}\right)$ and suction side vortex exit trajectory angle $\left(T L V / S S, \beta_{T L V}\right)$ of the scale M-fan at various tip clearances evaluated at their respective design point flow rates: $2 \mathrm{~mm}$ tip clearance $[\dot{V}=$ $\left.14.80 \mathrm{~m}^{3} / \mathrm{s}\right], 4 \mathrm{~mm}$ tip clearance $\left[\dot{V}=14.68 \mathrm{~m}^{3} / \mathrm{s}\right]$, and $6 \mathrm{~mm}$ tip clearance $[\dot{V}=$ $\left.14.57 \mathrm{~m}^{3} / \mathrm{s}\right]$

When comparing the TLV/SS exit trajectory angles, both the $4 \mathrm{~mm}$ and $6 \mathrm{~mm}$ tip clearance cases follow a similar trend. Both have similar initial exit trajectory angles which decrease over the blade chord. This could be as a result of the developing vortex core and blade suction side interaction. An increase in the tip clearance allows for a larger relative TLF velocity to pass through the tip gap. This in turn increases the momentum transfer to the TLV/SS thereby increasing both its size and intensity. The larger the vortex on the blade suction side, the further it will extend in the spanwise direction meaning it will be more influenced by the blade's suction side pressure field. This would cause the vortex to be 'drawn' closer to the blade surface which would explain the decrease in exit trajectory angle between 0.4 and 0.8 chord fractions. However, at this stage this is only considered a hypothesis.

Between 0.8 and 1.2 chord fractions, the exit trajectory angles of the $4 \mathrm{~mm}$ and $6 \mathrm{~mm}$ tip clearance cases dramatically increase before obtaining a similar exit trajectory gradient to that of the $2 \mathrm{~mm}$ tip clearance case after 1.2 chord fractions. Referring back to the helicity contour plots in Figure 5.1 on the blade's pressure side, it can be seen that the presence of the TLV/PS grows in significance from the $4 \mathrm{~mm}$ to $6 \mathrm{~mm}$ tip clearance cases whereas it is almost unidentifiable in the $2 \mathrm{~mm}$ 
CHAPTER 5. EFFECT OF TIP CLEARANCE AND TIP LEAKAGE VORTEX DEVELOPMENT

tip clearance case. A possible hypothesis that would explain both the increase in exit trajectory angle and increase in TLF velocity for the larger tip clearance case would be a greater interaction and subsequent merging of the two vortex structures.

\subsection{Blade surface pressure distribution}

Blade surface pressure distributions, $C p$, are considered for describing the TLV and flow field interaction near the blade tip. This is in order to determine the effect of the near surface flow field and vortex interaction with a reduction in tip clearance. The static pressure coefficient is defined as:

$$
C p=\frac{p-\bar{p}_{1}}{\frac{1}{2} \rho u^{2}}
$$

where $\bar{p}_{1}$ represents the average static pressure evaluated at the inlet and $u$ represents the blade's tangential velocity evaluated at the evaluation plane (Louw, 2015). The location of the evaluation plane is chosen such that the pressure distributions do not intersect the TLV core.

Figure 5.4 gives the blade pressure distributions of the scale M-fan at various tip clearances. The figure shows an increase in the pressure side $C p$ distributions with a reduction in tip clearance. These findings agree well with that found in literature (see Cumpsty and Storer, 1991). This would correspond to an improved relative velocity component due to a reduced effect from the created vortex structure interaction. 


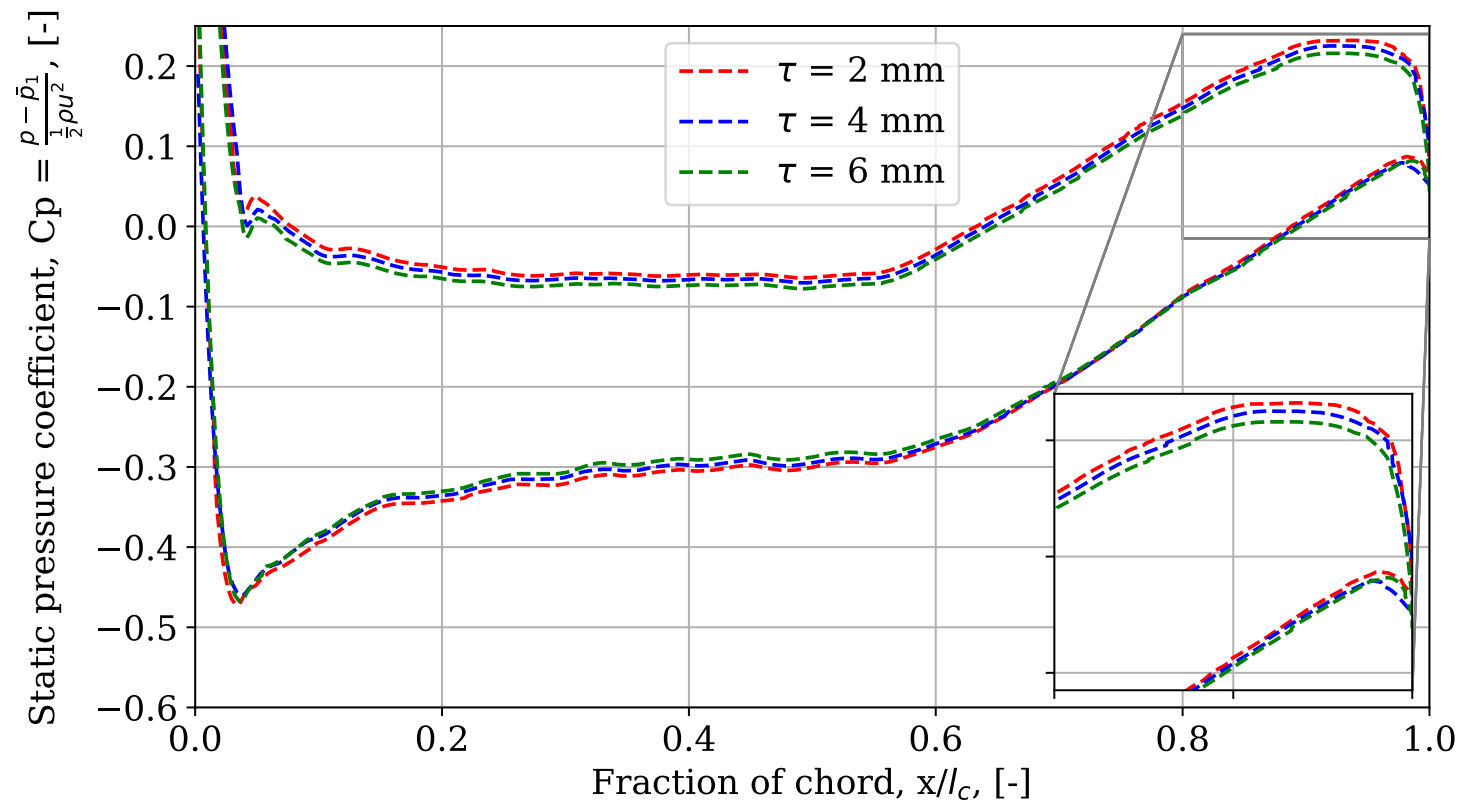

Figure 5.4: Chord-wise static pressure coefficient distributions $C p$ of the scale Mfan at various tip clearances evaluated at $91.6 \%$ blade span at the fan's design point flow rate

When examining the suction side pressure distributions, a consistent distribution is apparent after roughly 0.7 chord fractions for all tip clearance cases. This suggests that the suction side pressure distributions near the trailing edge of the blade are less affected by a reduction in tip clearance. A possible explanation for this could be due to the spanwise TLV development interacting with the suction side surface flow field.

\subsection{Chapter summary}

Fan performance characteristics and vortex development are evaluated at various tip clearances for the scale M-fan. The following points summarize the findings:

- A reduction in the tip clearance leads to an increase in fan performance characteristics across all flow rates. This is accompanied by a reduction in the relative TLF velocity within the tip gap. According to Venter and Kröger (1992), it is this reduction in TLF which reduces the associated pressure losses. 
CHAPTER 5. EFFECT OF TIP CLEARANCE AND TIP LEAKAGE VORTEX DEVELOPMENT

- Two predominant TLV structures are evident around the blade tip region (denoted TLV/PS and TLV/SS in this study). The change in exit trajectory angle of the TLV/SS decreases with a decrease in tip clearance. The interaction between these two vortex structures is found to decrease with a reduction in the tip clearance.

- An increase in the static pressure distributions on the blade's pressure side is apparent with a reduction in tip clearance. However, this is found not to be the case when examining the suction side pressure distributions near the blade's trailing edge. A likely explanation for this phenomenon has been provided, but at this stage it is only a hypothesis. 


\section{Chapter 6}

\section{Tip appendage design}

\subsection{Design considerations}

The constraints of the design are herewith listed:

1. It should be practical in its construction to modify existing fans through tip modification via attachment.

2. It should improve fan performance at the fan's design point.

Various tip configurations are reported in literature. These have been developed in an attempt to improve on an axial flow fan's noise and performance characteristics. The current study is only focused on the fan's performance improvement and will not consider fan noise characteristics. Considering the analysis conducted in the previous chapter, two predominant TLF characteristics are apparent with a reduction in tip clearance. These being a decrease in the TLF velocity within the tip gap and a decrease in the TLV exit trajectory angle along the blade chord. The next sections investigate the design for control of these two flow characteristics for the scale M-fan (details given in Table A.3 and hence forth referred to as the datum).

\subsection{Tip leakage flow control}

\subsubsection{Design}

Corsini et al. (2007b) found, through experimental testing, an improvement in fan static efficiency of up to 2.0 percentage points at the fan's design point flow rate with the addition of a constant thickness (CT) end-plate tip appendage. This was achieved by locally thickening the blade tip on the blade pressure side by a 
factor of three with respect to the maximum blade tip thickness. This dimension was chosen according to a reference radial dimension for control of the TLV. The introduction of such an end-plate was found to control the TLF by reducing its $W_{T L F}$ (Corsini et al., 2010$)$. However, this was accompanied by a reduction in fan static pressure rise of up to 6.4 percent.

As noted in Section 2.2.3. Denton (1993) found that if the tip clearance $\tau$ is less than 25 percent of the blade's thickness, a subsequent mixing of the TLF will occur with an increase in the static pressure and entropy across the tip gap. This mixing may hinder the TLF thereby resulting in its diminution. As a result, this thickness is considered as the lower limit for the end-plate design.

On the other hand, the size of the end plate suggested by Corsini et al. (2007b) (3times wider than the blade tip thickness) has been fixed as an upper limit for the current end-plate design. This is due to the modifications of the fan aerodynamic performance curves they obtained resulting in a reduction in the fan pressure rise characteristics. Consequently, this is not compatible with the present design goal.

The thickness limits for the proposed CT end-plate design are summarized as:

$$
t_{e p}= \begin{cases}3 \max \left(t_{\text {blade }}\right), & \text { maximum } \\ 4 \tau, & \text { minimum }\end{cases}
$$

As mentioned in Section 2.2.3, the pressure side edge of the end-plate design should be sharp as this produces weaker secondary vortices and induces a vena contracta effect within the tip gap entrance region. Corsini et al. $(2007 \mathrm{~b})$ only considers the effects of an end-plate on the blade's pressure side, yet, Cumpsty and Storer (1991) propose that it is the blade's suction side that is responsible for controlling the chord-wise TLF distribution. Consequently, this study conducts an investigation involving a CT end-plate on both the blade pressure side and suction side to evaluate its effect on fan performance.

Figure 6.1 illustrates the final CT end-plate designs, denoted CT/PS/24, CT/PS/47, and $\mathrm{CT} / \mathrm{SS} / 24$ in this study, with the unmodified datum blade shown for comparison. The blade tip is thickened locally, from blade chord, using the given criteria as set out in Equation 6.1. The corresponding minimum and maximum end-plate thicknesses for the fan datum are $24 \mathrm{~mm}$ and $47 \mathrm{~mm}$, respectively. The end-plates are extruded at a constant radius in order to maintain a constant tip clearance. The end-plate height (distance in the spanwise direction) is chosen such that a minimum of three cells span its length. 


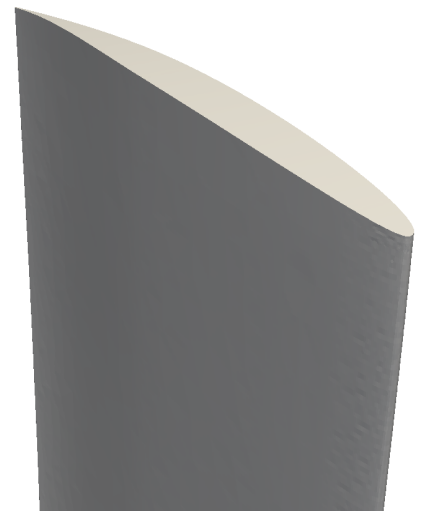

a) datum

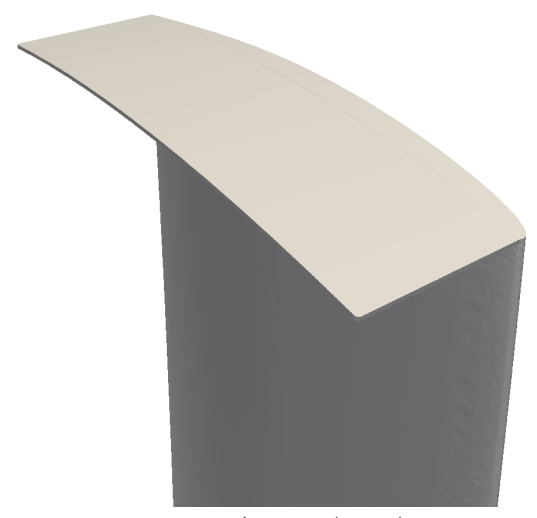

c) $\mathrm{CT} / \mathrm{PS} / 47$

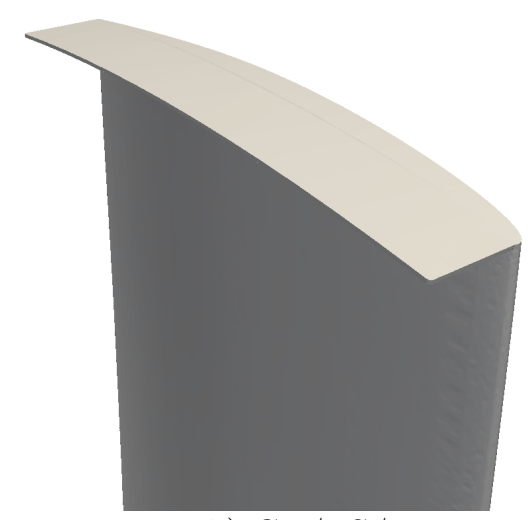

b) $\mathrm{CT} / \mathrm{PS} / 24$

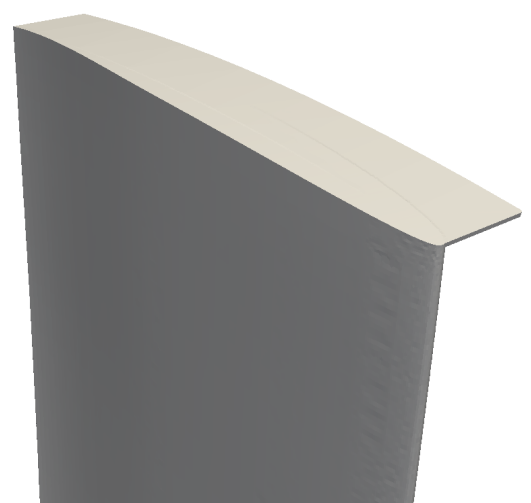

d) $\mathrm{CT} / \mathrm{SS} / 24$

Figure 6.1: Constant thickness (CT) end-plate designs: a) datum, b) $24 \mathrm{~mm}$ pressure side end-plate, c) $47 \mathrm{~mm}$ pressure side end-plate, and d) $24 \mathrm{~mm}$ suction side end-plate

\subsubsection{Aerodynamic performance analysis}

Figures 6.2 through 6.4 compare the fan performance characteristics of the CT end-plate designs to that of the datum fan with a $6 \mathrm{~mm}$ tip clearance. The results indicate improved performance characteristics of all pressure side CT end-plate designs with regards to fan total-to-static pressure rise at below design point flow rates. 


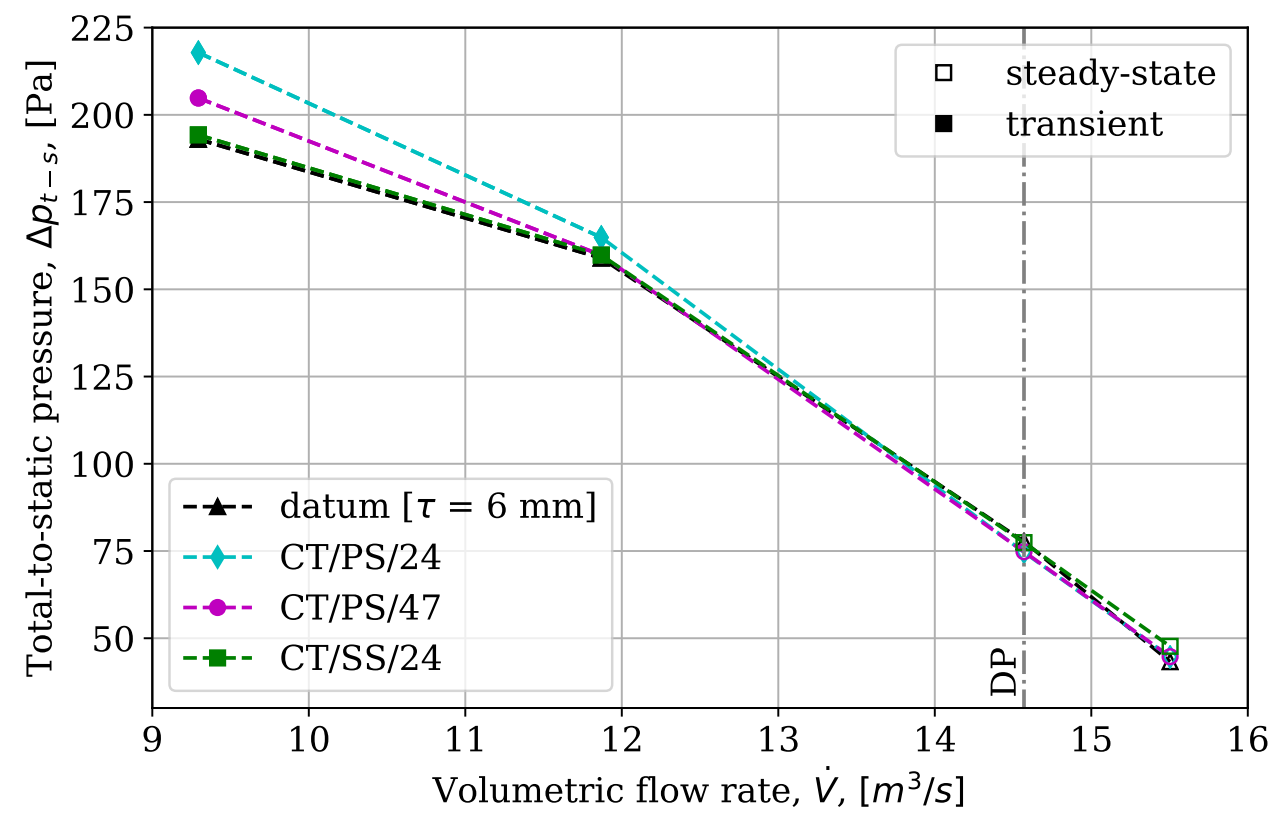

Figure 6.2: Constant thickness end-plate designs and datum fan numerical totalto-static pressure rise comparison

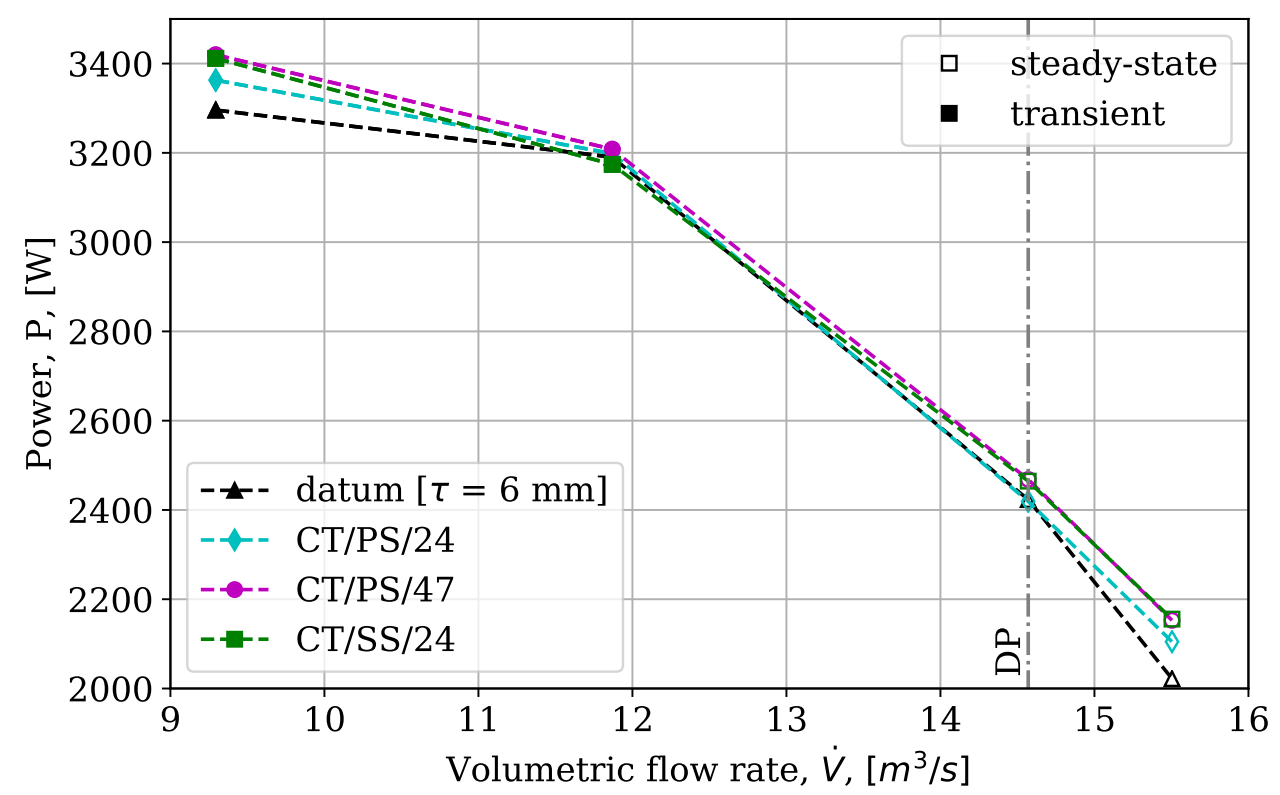

Figure 6.3: Constant thickness end-plate designs and datum fan numerical power comparison 


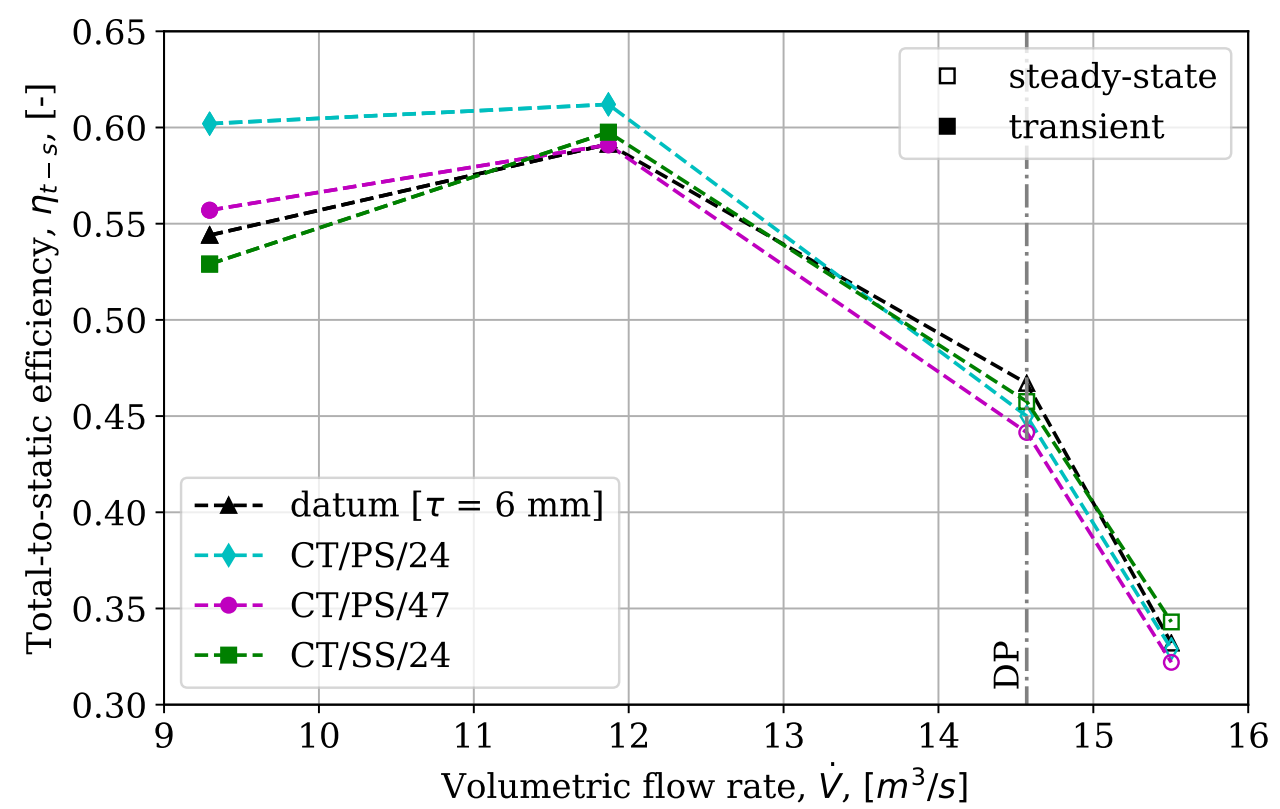

Figure 6.4: Constant thickness end-plate designs and datum fan numerical totalto-static efficiency comparison

The CT/PS/24 end-plate design is found to perform best at lower volumetric flow rates, with a 12.9 percent increase in total-to-static pressure rise and a 5.8 percentage point increase in total-to-static efficiency at the minimum evaluated $9.29 \mathrm{~m}^{3} / \mathrm{s}$ flow rate. The pressure side end-plate designs are found to increase the fan's peak efficiency plateau towards the rotor stall margin which concurs with that found by Corsini et al. (2007b).

As shown in Table 6.1, a decrease in fan performance characteristics is found for all CT end-plate designs at the fan's design point flow rate. A possible explanation for this could be due to the CT end-plate designs being less effective in controlling the $\mathrm{TLV} / \mathrm{SS}$ at relatively higher flow rates. At these operating conditions, a smaller vortex would be created due to a lower blade loading. The higher flow velocities would also mean that a greater power penalty would be incurred with the addition of the end-plate's additional surface area. These findings suggest the CT end-plate design to have a specific beneficial operating range whereby effective control of the TLV can be implemented. 
Table 6.1: Numerical fan performance characteristics of constant thickness endplate designs compared to fan datum evaluated at the fan's $14.57 \mathrm{~m}^{3} / \mathrm{s}$ design point flow rate for a $6 \mathrm{~mm}$ tip clearance

\begin{tabular}{cccc}
\hline & \multicolumn{3}{c}{ Variable } \\
Design & $\boldsymbol{\Delta} \boldsymbol{p}_{\boldsymbol{t}-\boldsymbol{s}}[\mathrm{Pa}]$ & Power $[\mathrm{W}]$ & $\boldsymbol{\eta}_{\boldsymbol{t}-\boldsymbol{s}}[-]$ \\
\hline datum $[\tau=6 \mathrm{~mm}]$ & 77.72 & 2423.18 & 0.467 \\
$\mathrm{CT} / \mathrm{PS} / 24$ & 74.66 & 2419.25 & 0.450 \\
$\mathrm{CT} / \mathrm{PS} / 47$ & 74.81 & 2468.41 & 0.441 \\
$\mathrm{CT} / \mathrm{SS} / 24$ & 77.41 & 2464.56 & 0.458 \\
\hline
\end{tabular}

A decrease in fan pressure and fan efficiency characteristics across all evaluated flow rates is found with an increase in the pressure side end-plates thickness from $24 \mathrm{~mm}$ to $47 \mathrm{~mm}$. This is predominately due to the increased power requirement of the larger end-plate design. This suggests that an operating point specific endplate thickness exists in order to control the TLV while minimizing the induced drag associated with an increased surface area.

With the addition of the suction side end-plate $\mathrm{CT} / \mathrm{SS} / 24$ design, the results indicate a 1.1 percentage point increase in fan total-to-static efficiency at the evaluated operating point greater than that of design. However, a deterioration in fan performance characteristics is found when considering all other evaluated flow rates for this design. This could be due to higher relative flow velocities being present on the blade suction side, thereby significantly increasing the designs power requirement. This is reflected in Figure 6.3. It is for this reason that no further $\mathrm{CT} / \mathrm{SS}$ end-plate designs are tested.

\subsubsection{Vortex analysis}

Figure 6.5 shows the helicity contour plots of the three CT end-plate designs at the fan's design flow rate for a $6 \mathrm{~mm}$ tip clearance. It should be mentioned that the end-plate geometries are not included in the figure; instead the datum fan blade is used to visualize the blade with an outline of the end-plate geometry. This is purely for illustrative purposes as the end-plate designs would otherwise obscure the view of the contour plots. All three designs show the same presence of two clockwise rotating vortex structures, that being TLV/PS and TLV/SS, as seen previously in the datum fan case in Section 5.3 . 


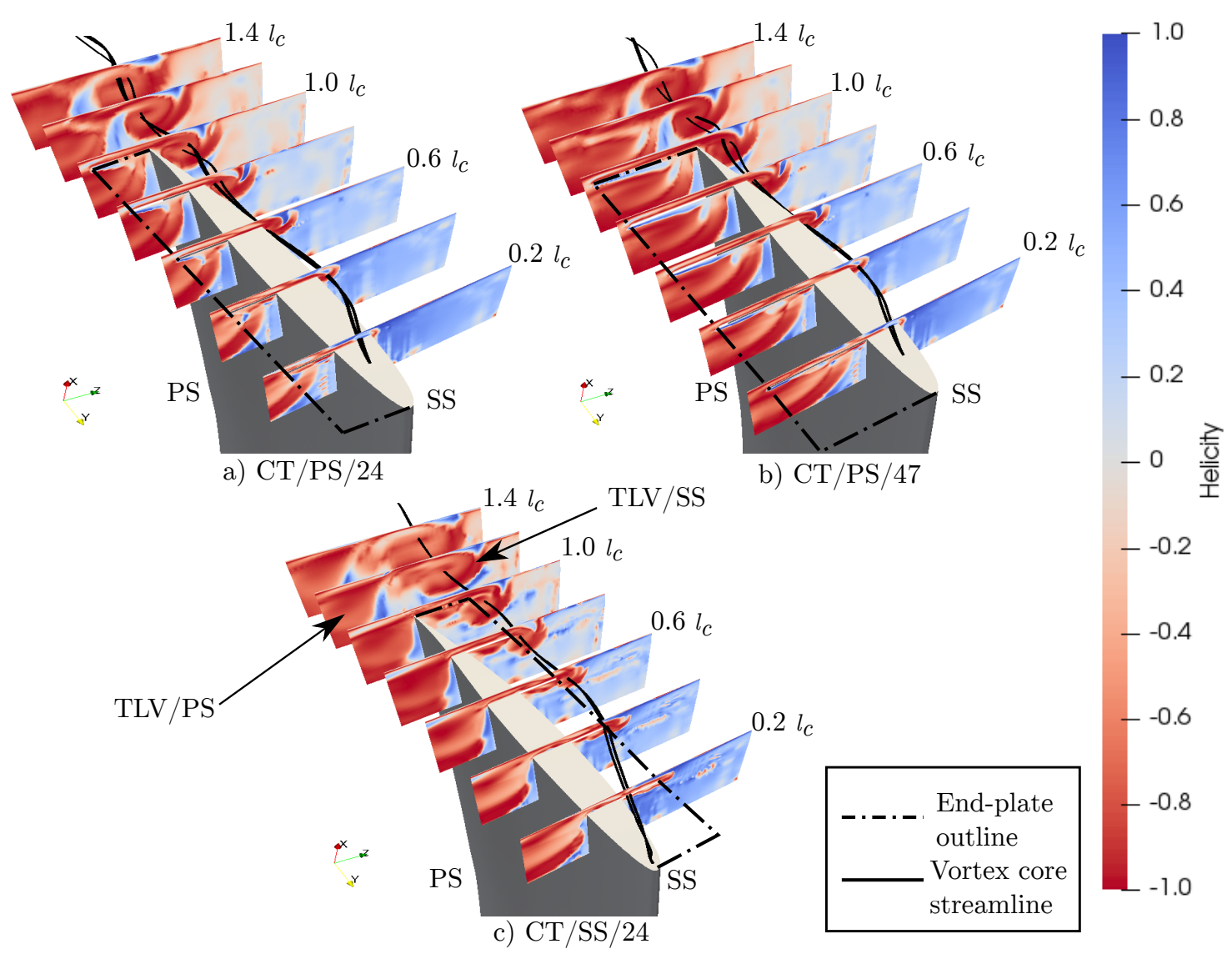

Figure 6.5: Normalized helicity $H_{\text {norm }}$ cross sectional contours of constant thickness end-plate designs at blade tip with suction side vortex (TLV/SS) visualization evaluated at the fan's $14.57 \mathrm{~m}^{3} / \mathrm{s}$ design point flow rate for a $6 \mathrm{~mm}$ tip clearance: a) $24 \mathrm{~mm}$ pressure side end-plate, b) $47 \mathrm{~mm}$ pressure side end-plate and c) $24 \mathrm{~mm}$ suction side end-plate

Comparing the helicity contour plots of the CT/PS $24 \mathrm{~mm}$ and $47 \mathrm{~mm}$ end-plates in Figure 6.5, a negligible difference is found in terms of both the size and shape of both vortex structures. The TLV/SS of both cases initiate at similar locations (that being between 0.2 and 0.4 chord fractions). The contours surrounding the pressure side end-plate's surface suggest the presence of uniform flow. This could be as a result of the end-plates acting as physical barriers to the TLV/PS rotation. When comparing the helicity contours of the pressure side end-plate designs to that of the datum fan in Figure 5.1k, no noticeable difference is found in vortex size. This suggests that the addition of the CT end-plate design is unable to effectively control the TLV at relatively higher flow rates. 
The addition of an end-plate on the blade suction side extends the TLV/SS core further away from the blade surface when compared to the other designs. This causes the vortex to become more elongated and elliptical in shape while also increasing its size when compared to the datum.

Figure 6.6 shows a comparison of the TLF velocity and TLV/SS exit trajectory angle along the blade chord for the various CT end-plate designs at the fan's design point flow rate. The findings show a overall reduction in the TLF velocity along the blade chord of all the CT end-plate designs when compared to the datum. The average TLF velocity reduction along the blade chord is found to be 6.8 percent for both the $\mathrm{CT} / \mathrm{PS} / 47$ and $\mathrm{CT} / \mathrm{SS} / 24$ end-plate designs when compared to the datum.

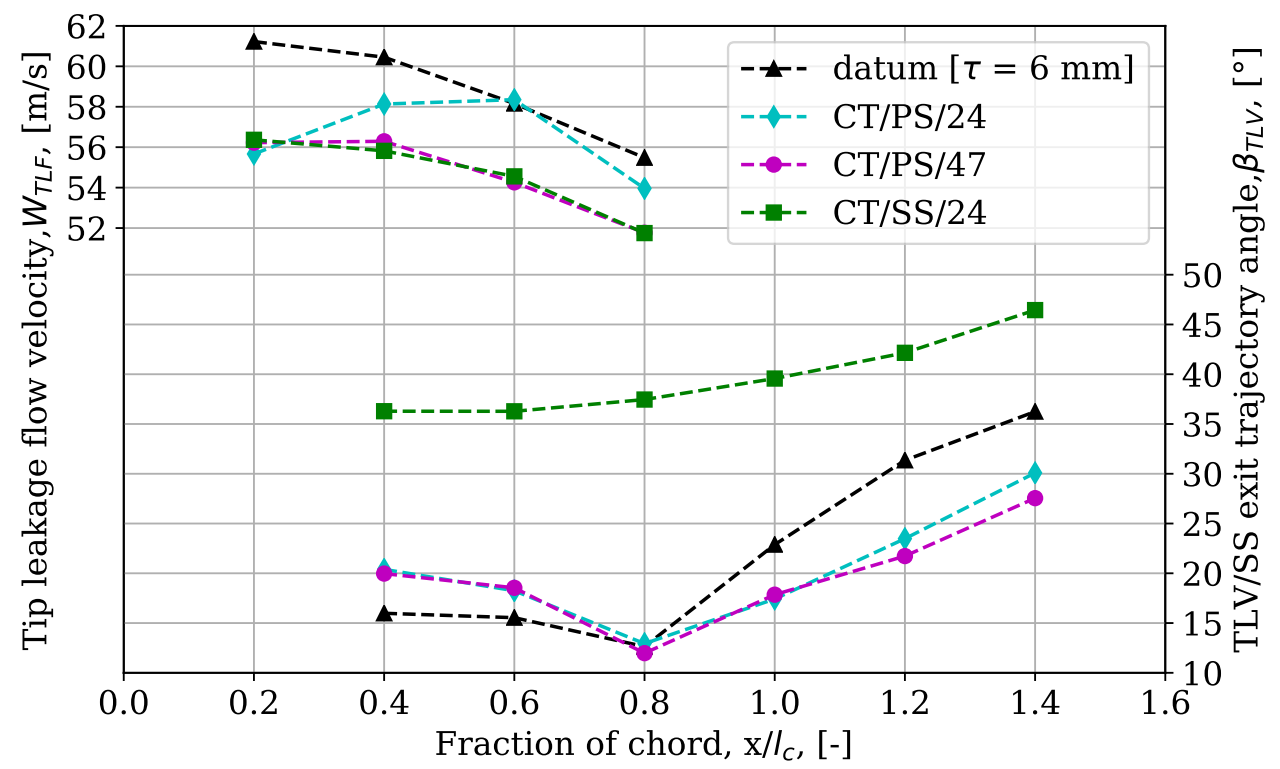

Figure 6.6: Relative tip leakage flow velocity $\left(W_{T L F}\right)$ and suction side vortex exit trajectory angle $\left(T L V / S S, \beta_{T L V}\right)$ of constant thickness end-plate designs evaluated at the fan's $14.57 \mathrm{~m}^{3} / \mathrm{s}$ design point flow rate for a $6 \mathrm{~mm}$ tip clearance

When examining the TLV/SS exit trajectory angles in the same figure, it can be seen that both CT/PS end-plate designs reduce the TLV/SS exit trajectory angle beyond the trailing edge of the blade, with a further reduction as the end-plate thickness is increased. When considering the suction side CT/SS/24 end-plate design, a significant increase in the TLV/SS exit trajectory angle becomes apparent as mentioned previously. This is due to the suction side end-plate design extending 
the vortex inception point thereby forcing it to have a larger exit trajectory angle.

However, despite the end-plate designs offering a reduction in the TLF velocity and both an increased and decreased TLV/SS exit trajectory angle, none of the designs improve the fan's performance characteristics at the design point flow rate. This suggests that there may exist another metric which could further aid in characterizing the leakage flow.

\subsubsection{Blade surface pressure distribution}

Figure 6.7 illustrates the blade surface pressure distributions, $C p$, of the CT endplate designs compared to the datum fan. When examining the various pressure distributions towards the blade's trailing edge, a decrease in blade loading is found through the addition of all CT end-plate designs when compared to the datum. A reduction in blade loading near the blade tip suggests a reduction in fan performance as the tip region is consequently more affected by the TLV. These findings are supported when referring back to the aerodynamic performance analysis conducted in Section 6.2.2.

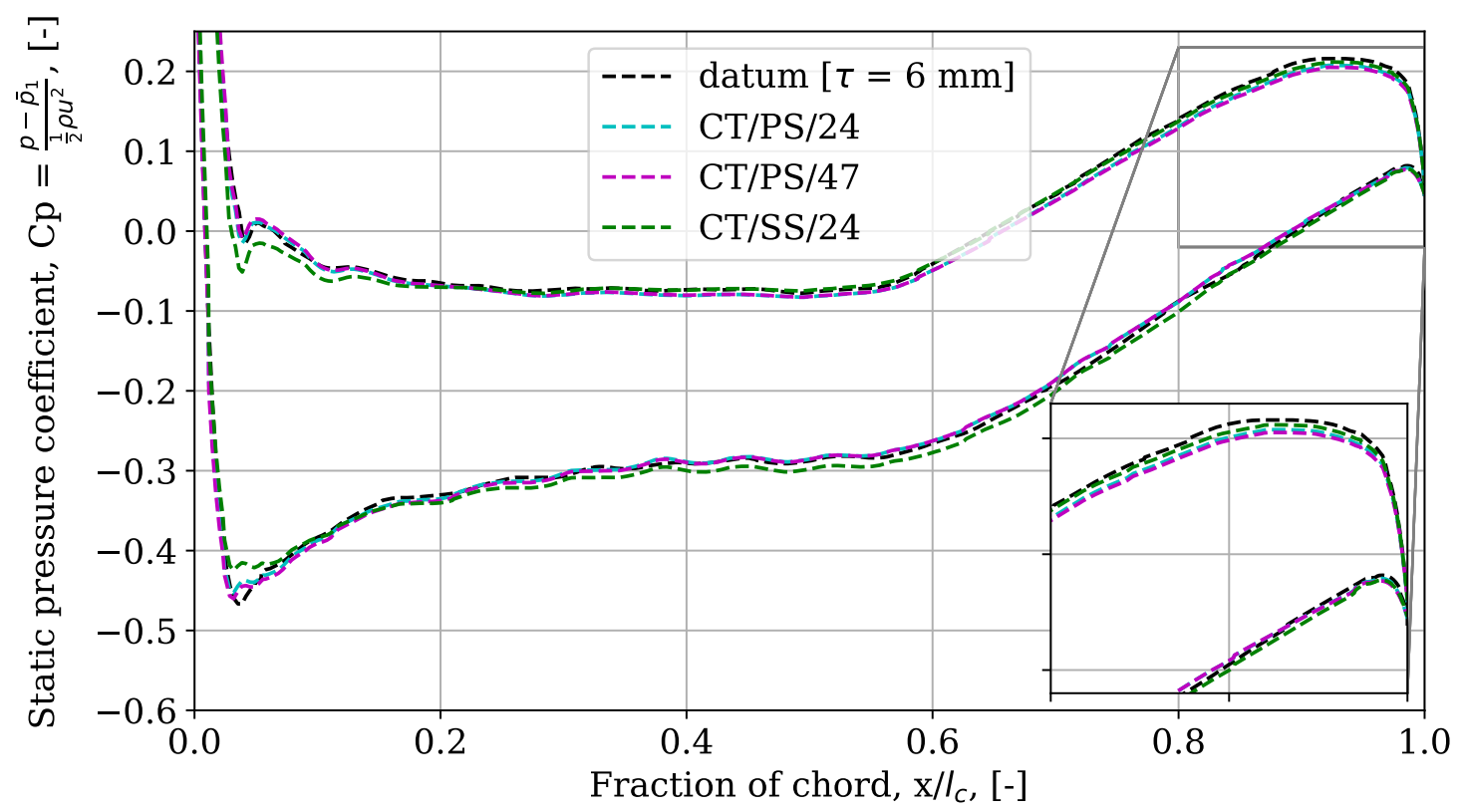

Figure 6.7: Chord-wise static pressure coefficient distributions $C p$ of constant thickness end-plate designs compared to fan datum evaluated at $91.6 \%$ blade span at the fan's design point flow rate 
When considering the $\mathrm{CT} / \mathrm{SS} / 24$ end-plate design, an increase in blade loading is evident around mid-chord when compared to the datum. This would suggest that an increase in aerodynamic performance should be apparent, however, the opposite is found true when comparing the numerical analysis results conducted in Section 6.2.2.

These findings suggest that an aft loaded blade is desirable when optimizing blade loading distributions for fan performance enhancement. Supporting literature is found to concur with the former when considering loss generation associated to compressor stage performance, which finds delayed TLV formation with a reduced mixed-out TLF at the outlet (Tiralap et al., 2017).

\subsection{Tip leakage vortex exit trajectory angle control}

\subsubsection{Design}

A decrease in the TLV/SS exit trajectory angle correlates with a reduction in tip clearance. The blade-to-blade vortex interaction affects the relative flow velocity angles, thus affecting the performance characteristics of the blade. As mentioned in Section 5.4, the largest TLV/SS exit trajectory gradient of the $6 \mathrm{~mm}$ tip clearance case occurs between 0.8 and 1.2 chord fractions. Extending the trailing edge (TE) of the datum fan within this region (between 1.0 and 1.2 chord fractions) through the use of an end-plate could provide for the reduction and subsequent control of the TLV/SS exit trajectory angle. The novel end-plate concept would extend in the spanwise direction and cover the size of the vortex to be controlled (determined from the helicity contour plots presented in Section 5.3).

The advantage of this design, when compared to that of the CT end-plate designs, is a reduction in the fan's power requirement due to a reduction in the introduced surface area. This gives the TE design a higher potential in improving the fan's efficiency when compared to the CT end-plate designs.

Based on the analysis performed in Chapter 5, the end-plate dimension requirements for the proposed TE end-plate design are summarized below.

$$
\begin{aligned}
& h_{e p}=0.03 D_{\text {rotor }} \\
& l_{\text {ep }}=0.2 l_{c}
\end{aligned}
$$

Figure 6.8 illustrates the TE end-plate design. The end-plates are extruded such that a constant tip clearance is maintained with sharp edges (as done for the CT 
end-plate designs). For the $6 \mathrm{~mm}$ tip clearance datum case at the fan's $14.57 \mathrm{~m}^{3} / \mathrm{s}$ design flow rate, the dimensions of the end-plate height $h_{e p}$ (measured from blade tip) and length $l_{e p}$ (measured from blade trailing edge) are found to be $46 \mathrm{~mm}$ and $35 \mathrm{~mm}$, respectively, as these are found to be the dimensions of the desired vortex to be controlled within the specified region of such control. Likewise with that of the CT end-plate design, the TE end-plate thickness $\left(t_{e p}\right)$ is chosen such that a minimum of three cells span its length.
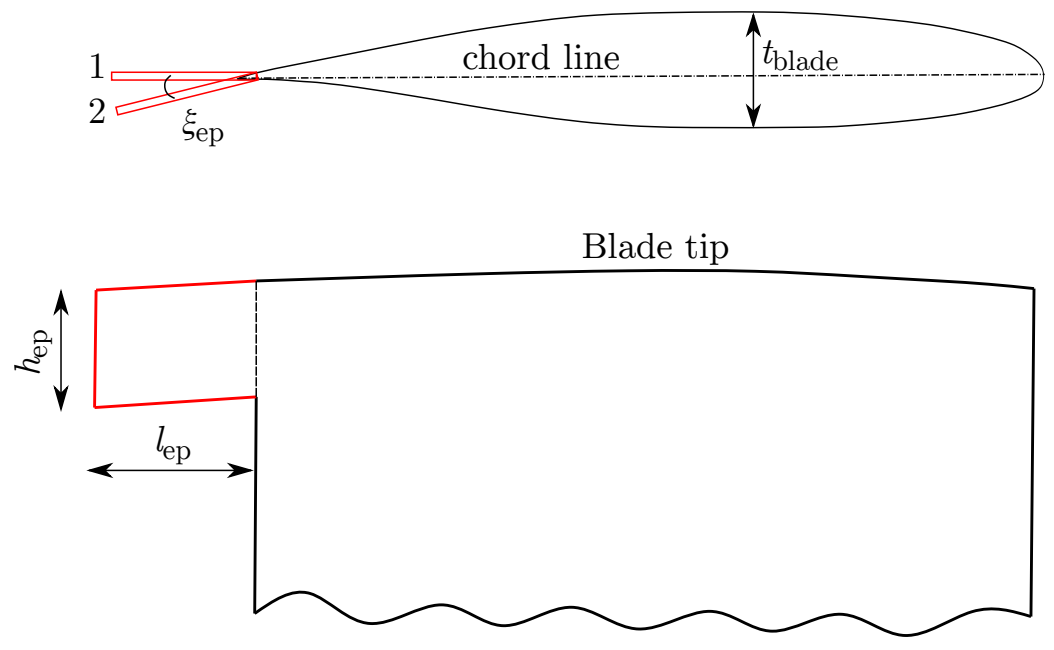

Figure 6.8: Trailing edge (TE) end-plate design

The angle $\zeta_{e p}$ (between points 1 and 2 in the figure) is the setting angle of the end-plate relative to the blade chord. As the setting angle is increased from a zero angle, the end-plate will deflect the pressure side flow, thereby deflecting the TLV/PS further downstream of the blade row. This should reduce the TLV/SS exit trajectory angle, thereby reducing its effect on the blade's relative velocity angles.

An additional benefit through the introduction of the TE end-plate design is the creation of a localized aft pressure differential near the blade tip as a result of the pressure side flow deflection. This in turn should shift the blade's peak blade loading distribution further towards the blade's trailing edge.

To determine a range of setting angles for the proposed TE end-plate designs, flat plate theory is used in order to reduce the inherent complexity of the system. This is to acquire an initial range of values to determine feasibility of the design. Figure 6.9 illustrates experimental lift $\left(C_{L}\right)$ and mid-chord moment coefficients $\left(C_{M, m c}\right)$ at various angles of attack (AoA) for a flat plate of similar size to that 
of the proposed design and at a similar Reynolds number to the designs intended operating point.
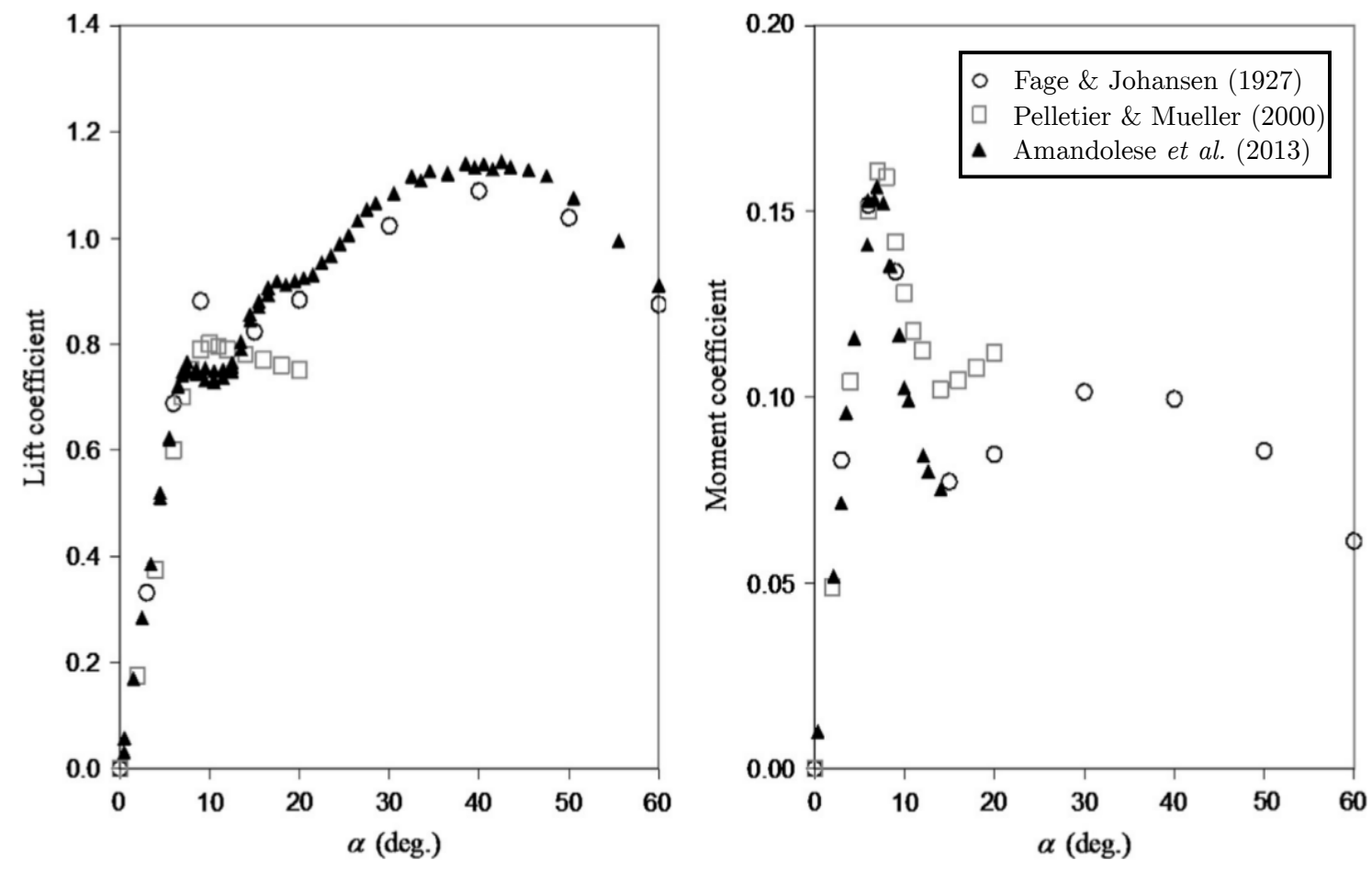

Figure 6.9: Flat plate lift coefficient and moment coefficient about the mid-chord versus angle of attack (adapted from Amandolese et al., 2013)

Considering the lift coefficients (left), three $C_{L}$ plateaus are apparent, located at around $10^{\circ}, 20^{\circ}$ and $40^{\circ}$ AoA. Comparing the relevant moment coefficients located at the same locations right of the figure, it can be seen that the $10^{\circ}$ AoA case offers the greatest moment about its mid-chord while an AoA between $10^{\circ}$ and $20^{\circ}$ offers the lowest. This is import as it is desirable to maximize the $C_{L}$ value without causing flow separation, while minimizing the moment force exerted on the end-plate to reduce the fan's power requirement. For this reason, only the $10^{\circ}$ and $20^{\circ}$ AoA cases are chosen to determine an operational range for the proposed end-plate concept.

The end-plate setting angles are determined based on the desired end-plate AoA and relative flow angles of the $6 \mathrm{~mm}$ tip clearance datum fan case. A $0^{\circ}$ end-plate setting angle is additionally chosen to determine the effect of extending the blade's trailing edge along the blade chord. The final three TE end-plate configurations are denoted TE/PS/0deg, TE/PS/4deg, and TE/PS/15deg. 


\subsubsection{Aerodynamic performance analysis}

Figures 6.10 through 6.12 compare the fan performance characteristics of the TE end-plate designs to that of the datum fan with a $6 \mathrm{~mm}$ tip clearance. The same operational points have been chosen for evaluation as done for the CT end-plate designs. The results indicate maintained or otherwise improved performance characteristics of all TE end-plate designs with regards to fan total-to-static pressure rise across all evaluated flow rates.

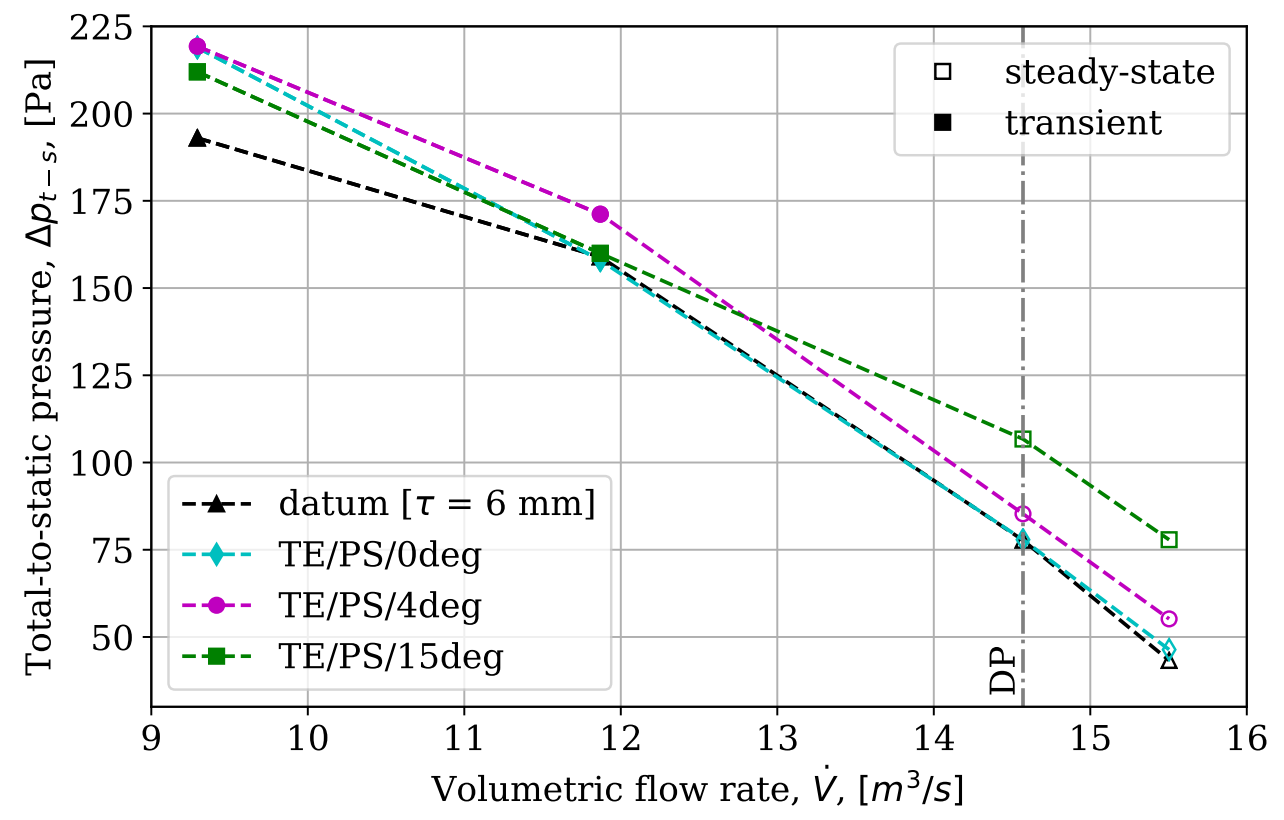

Figure 6.10: Trailing edge end-plate designs and datum fan numerical total-tostatic pressure rise comparison 


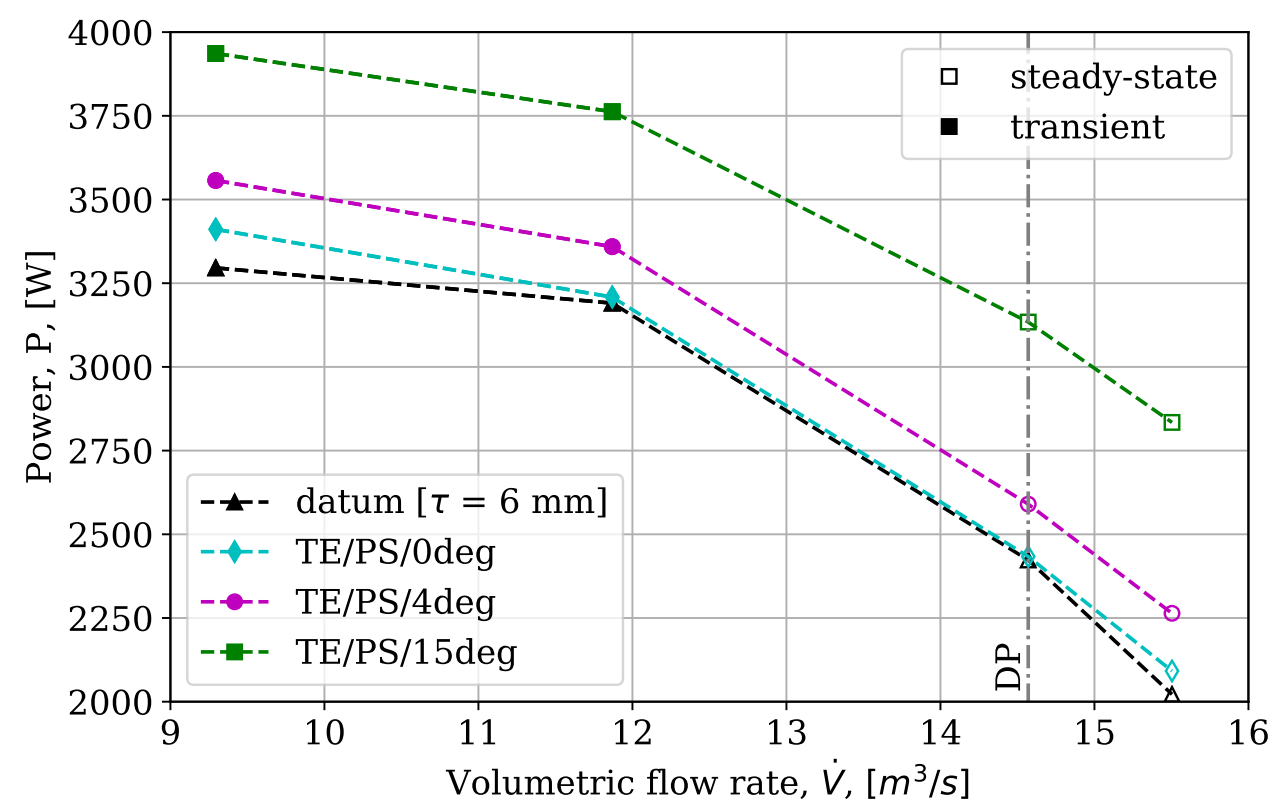

Figure 6.11: Trailing edge end-plate designs and datum fan numerical power comparison

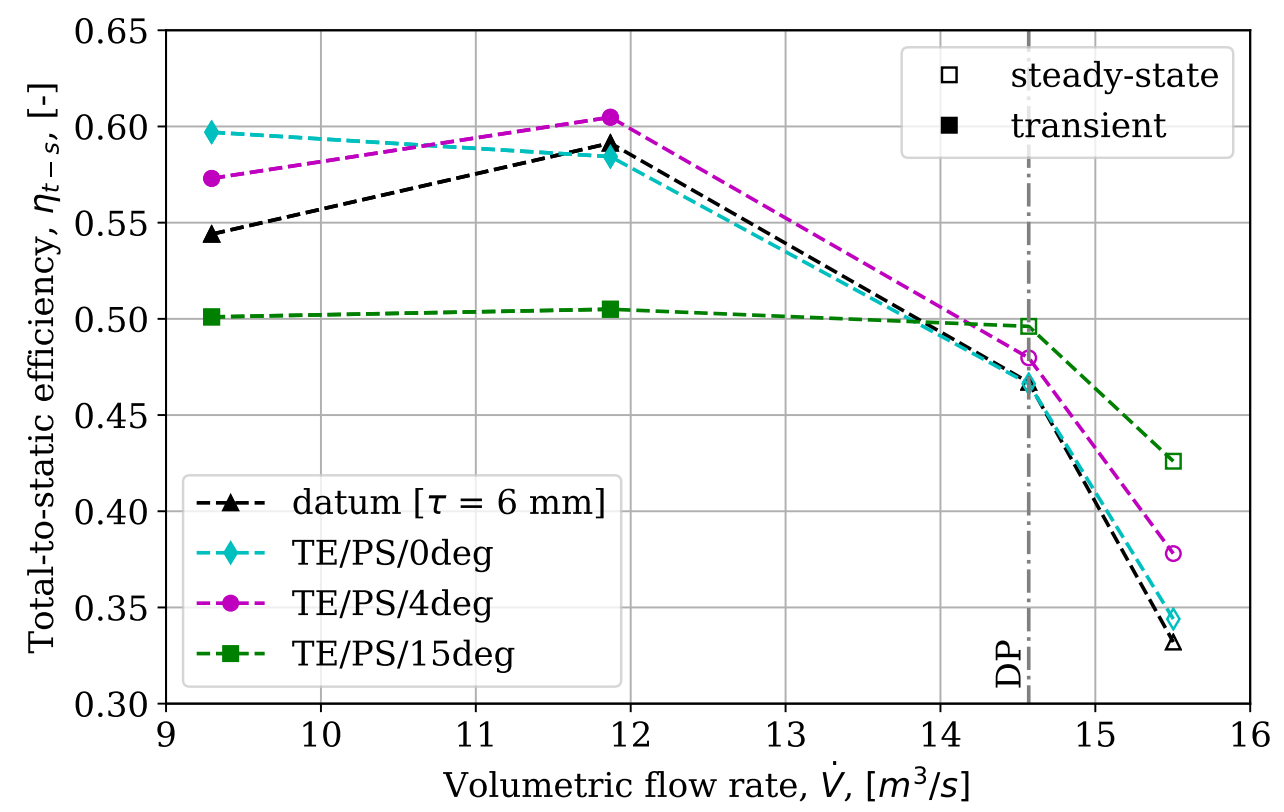

Figure 6.12: Trailing edge end-plate designs and datum fan numerical total-tostatic efficiency comparison

Table 6.2 quantitatively indicates that the TE/PS/15deg end-plate design is found 
to perform significantly better than that of the other end-plate designs at the fan's design point flow rate, with an indicated increase of 37.3 percent in total-to-static pressure rise and a 2.9 percentage point increase in total-to-static efficiency.

Table 6.2: Numerical fan performance characteristics of trailing edge end-plate designs compared to fan datum evaluated at the fan's $14.57 \mathrm{~m}^{3} / \mathrm{s}$ design point flow rate for a $6 \mathrm{~mm}$ tip clearance

\begin{tabular}{cccc}
\hline & \multicolumn{3}{c}{ Variable } \\
Design & $\boldsymbol{\Delta} \boldsymbol{p}_{\boldsymbol{t}-\boldsymbol{s}}[\mathrm{Pa}]$ & Power $[\mathrm{W}]$ & $\boldsymbol{\eta}_{\boldsymbol{t}-\boldsymbol{s}}[-]$ \\
\hline datum $[\tau=6 \mathrm{~mm}]$ & 77.72 & 2423.18 & 0.467 \\
TE/PS/0deg & 77.89 & 2433.51 & 0.466 \\
TE/PS/4deg & 85.29 & 2590.08 & 0.480 \\
TE/PS/15deg & 106.73 & 3134.06 & 0.496 \\
\hline
\end{tabular}

However, a significantly reduced total-to-static efficiency is found for the TE/PS/15deg end-plate design at lower than design point flow rates. This is likely a result of increased drag due to the severe end-plate setting angle which would explain the increased power requirement apparent in Figure 6.11. When considering the lower extreme of the end-plate setting angles, the TE/PS/0deg design is found to perform best at the lower $9.29 \mathrm{~m}^{3} / \mathrm{s}$ operating flow rate with an increase of approximately 13.5 percent in total-to-static pressure rise and a 5.2 percentage point increase in total-to-static efficiency. The TE/PS/4deg end-plate design is found to provide an intermediate between the designs, with improved performance characteristics across all evaluated flow rates.

These findings suggest that an operating point specific end-plate setting angle exists whereby effective control of the TLV exit trajectory angle can be implemented; however, this also indicates that the chosen TE end-plate setting angle range is sufficient in describing the performance bounds of the design. The TE/PS/0deg and TE/PS/4deg end-plate designs indicate an improved rotor stall efficiency plateau, as evident in the CT end-plate designs. Overall, the TE/PS/4deg end-plate design is found to perform best over the evaluated stable operating range.

\subsubsection{Vortex analysis}

Figure 6.13 shows the helicity contour plots of the three TE end-plate designs at the fan's design flow rate for a $6 \mathrm{~mm}$ tip clearance. When comparing the TE/PS/0deg design contours to that of the datum fan in Figure 5.1c, no significant change is 
evident between the two interacting vortex structures in terms of both size and intensity.

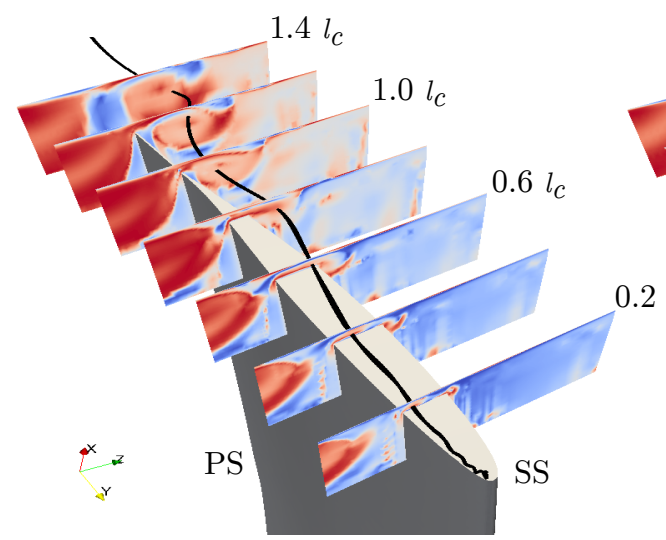

a) TE/PS/0deg

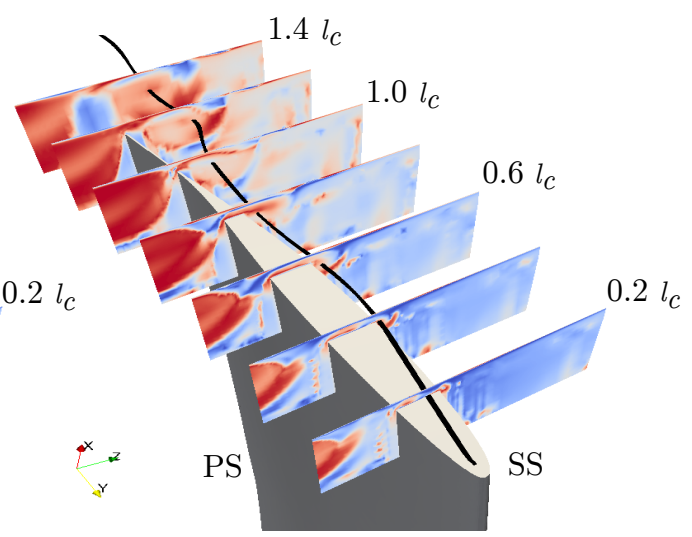

b) $\mathrm{TE} / \mathrm{PS} / 4 \mathrm{deg}$

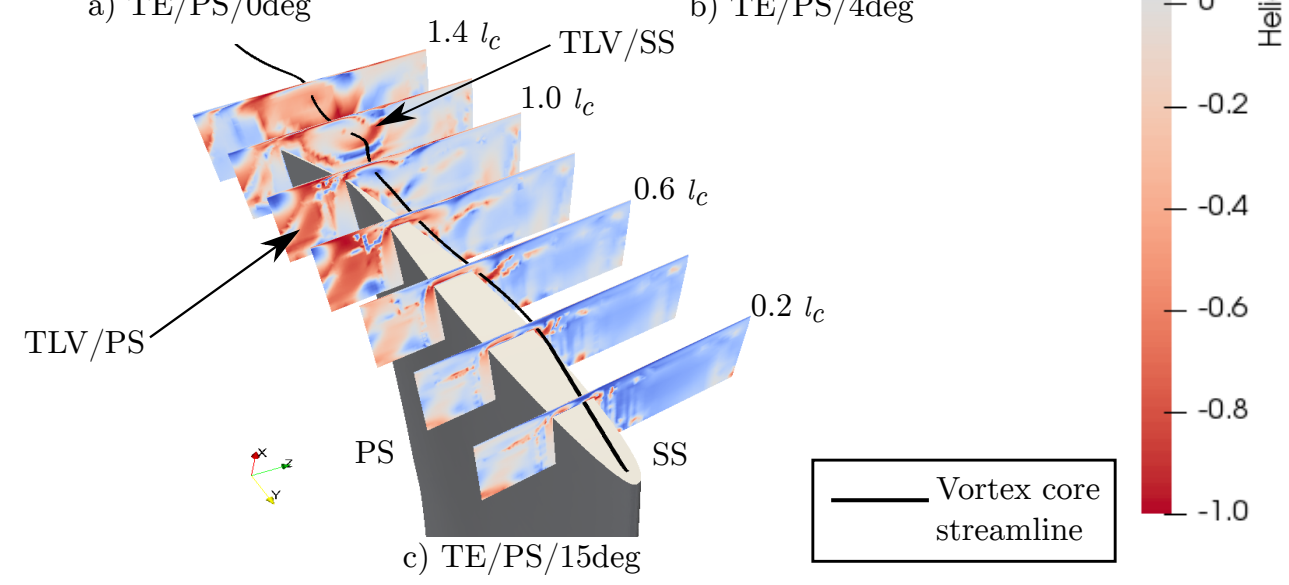

Figure 6.13: Normalized helicity $H_{\text {norm }}$ cross sectional contours of trailing edge end-plate designs at blade tip with suction side vortex (TLV/SS) visualization evaluated at the fan's $14.57 \mathrm{~m}^{3} / \mathrm{s}$ design point flow rate for a $6 \mathrm{~mm}$ tip clearance: a) $\zeta_{e p}=0^{\circ}$, b) $\zeta_{e p}=4^{\circ}$, and c) $\zeta_{e p}=15^{\circ}$

However, when comparing the contours between all the TE designs in Figure 6.13a through c, a noticeable reduction in the size of the TLV/PS structure is apparent with an increase in the TE end-plate's setting angle. This reduction is comparable to that experienced with a reduction in tip clearance. Likewise with the $2 \mathrm{~mm}$ tip clearance datum case, the TLV/PS is almost unrecognisable in the TE/PS/15deg case. This suggests that the TE end-plate design is effective in reducing the TLV/SS exit trajectory angle thereby subsequently reducing the interaction between the two vortex structures TLV/SS and TLV/PS. 
Figure 6.14 shows a comparison of the TLF velocity and TLV/SS exit trajectory angle along the blade chord for the various TE end-plate designs at the fan's design flow rate. As shown, the TE end-plate designs do not reduce the TLF velocity but rather increase it towards the blade's trailing edge as the TE end-plate setting angle is increased. This seems counter-intuitive as it is thought that the TLF is one of the predominant driving mechanisms behind the formation of the TLV and its reduction is thought to be desirable in reducing the associated mixing losses.

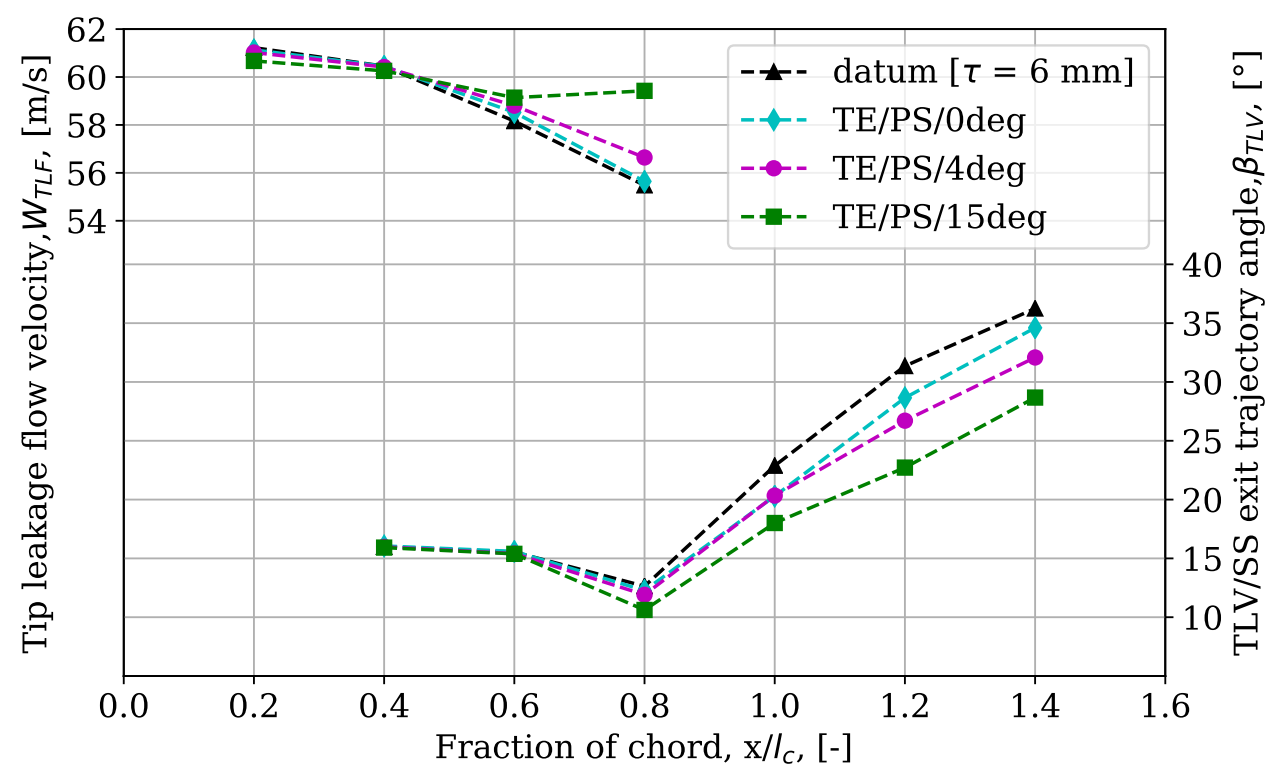

Figure 6.14: Tip leakage flow velocity $\left(W_{T L F}\right)$ and suction side vortex exit trajectory angle $\left(T L V / S S, \beta_{T L V}\right)$ of trailing edge end-plate designs evaluated at the fan's $14.57 \mathrm{~m}^{3} / \mathrm{s}$ design point flow rate for a $6 \mathrm{~mm}$ tip clearance

The increase in TLF velocity could be due to the local aft pressure differential being created as a result of the introduction of the TE end-plate concept. When examining the TLV/SS exit trajectory angles, a near linear reduction is found aft of the blade with an increase in the TE end-plate setting angle. This suggests that the addition of the TE end-plate design is an effective tool in controlling the $\mathrm{TLV} / \mathrm{SS}$ exit trajectory angle.

\subsubsection{Blade surface pressure distribution}

Figure 6.15illustrates the blade surface pressure distributions, $C p$, of the TE endplate designs compared to the datum fan at design point. The findings indicate that both the TE/PS/4deg and TE/PS/15deg end-plate designs increase the blade 
loading across the aft section of the blade tip, with the TE/PS/15deg design performing significantly better than the other designs. This suggests that the TE end-plate design is effective in shifting the blade loading distributions further towards the blade's trailing edge.

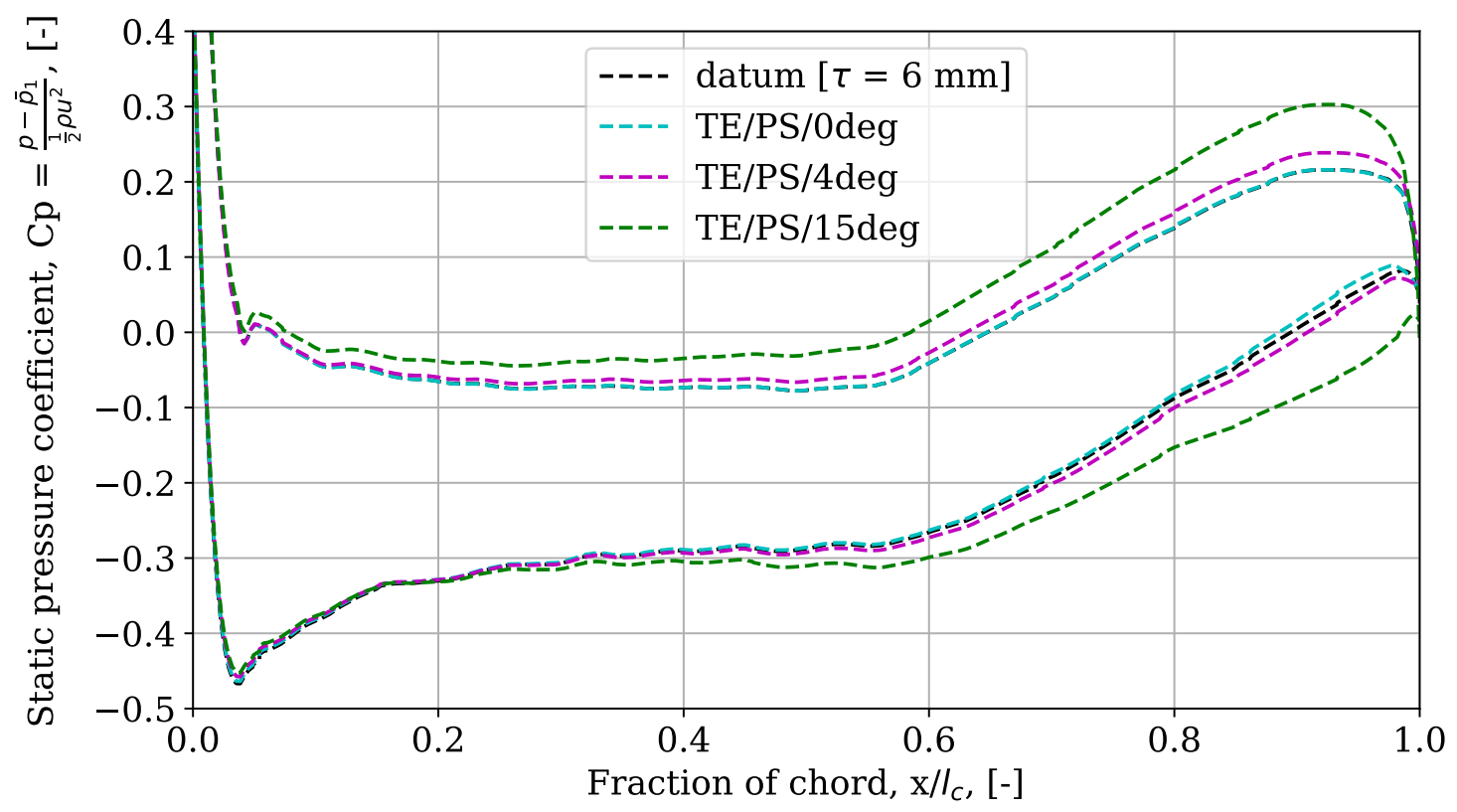

Figure 6.15: Chord-wise static pressure coefficient distributions $C p$ of trailing edge end-plate designs compared to fan datum evaluated at $91.6 \%$ blade span at the fan's design point flow rate

As previously mentioned, aft blade loading is thought to delay the TLV formation and reduce the mixed-out TLF at the rotor outlet (Tiralap et al. 2017). Supporting evidence from Section 6.3.2 indicates that an aerodynamic gain is indeed associated with the aforementioned TE end-plate designs.

\subsection{Chapter summary}

The current chapter details two end-plate designs for the control and mitigation of the TLF (and subsequent TLV formation) for a rotor only axial flow fan. The following points summarize the findings:

- All pressure side CT end-plate designs indicate improved fan performance characteristics at below design point flow rates with an increased efficiency plateau towards the rotor stall margin. The suction side end-plate design 
is found only to improve the fan performance characteristics at higher than design point flow rates, although only marginally. All designs are found to reduce the relative TLF velocity within the tip gap at the fan's design point flow rate; however, none are found to improve the fan's performance characteristics at this operating point.

- The TE end-plate designs are found to control the TLV formation through a reduction of the TLV/SS exit trajectory angle and subsequent reduction of the blade-to-blade vortex interaction. An aerodynamic performance analysis of the design indicates improved fan performance characteristics across all evaluated flow rates, including the design flow rate. Additionally, the design is found to shift the blade's peak loading distribution further aft along the blade chord, which has a beneficial effect on the mixing of the TLF and subsequent TLV formation. These findings suggest that a specific end-plate setting angle exists whereby effective control of the TLV exit trajectory angle can be implemented. 


\section{Chapter 7}

\section{Design screening}

As indicated for both design analyses conducted in Chapter 6, specific end-plate operating conditions exist whereby effective control of the TLV can be implemented. As it is desirable to determine such control which maximizes the performance of the fan, optimization is considered in this investigation for the further development of the end-plates. This is due to the uncertainty associated with knowing the exact influence that the various end-plate parameters have on the fan's performance characteristics, as well as which combination best provides for optimal performance. This chapter presents the fundamental quantities used in defining the current optimization procedure.

\subsection{Design selection}

As both designs aim to achieve the same desired outcome, that being the improvement of the fan's aerodynamic performance characteristics, selection of the most promising design is required for the optimization procedure. Comparing the various designs in Chapter 6, it is evident that the TE end-plate design is superior to that of the CT end-plate design with regards to aerodynamic performance. This is due to the design's inherent ability to improve the fan's performance characteristics across the entire evaluated stable operating range (including that of the design point). This is found not to be the case for the CT end-plate design which is only able to improve the fan's performance characteristics at below design point flow rates.

Referring back to Section 6.1, one of the requirements of the design is that it should improve the performance at the fan's design point flow rate. For this reason, the $\mathrm{TE}$ end-plate design is chosen for further development as it meets this requirement. 


\subsection{Optimization problem definition}

As introduced in Section 2.6, definition of the optimization problem forms a critical step in the optimization procedure. As such, the following sections discuss the functions used in the optimization routine.

\subsubsection{Objective function}

In order to formulate the objective function to be used in the current optimization routine, real-world performance metrics must be used to provide meaningful feedback for later experimental verification. As previously mentioned, one of the predominate requirements of the end-plate design is that it should improve the performance at the fan's design point flow rate.

From the aerodynamic performance analysis conducted in Section 6.3.2, the most representative performance characteristic must be that of the end-plate's total-tostatic efficiency. This is due to the metric being able to give the relative correlation between the fan's pressure and power characteristics, which is consequently also found to be able to capture the adverse effects caused by large end-plate setting angles (unlike that of the other performance characteristics). From this, the first objective function considers the total-to-static efficiency improvement of the datum fan at the fan's design point flow rate, defined as:

$$
f_{1}(\mathbf{x})=\frac{\eta_{D P}-\eta_{D P}^{*}}{\eta_{D P}}
$$

where $*$ represents the optimized value and a negative objective function value indicates a higher design point efficiency improvement.

As noted by van der spuy (2011), strong cross-winds commonly encountered during operation of the ACC cause distorted inlet flow conditions which adversely affect the performance of the fan and consequently lowers volume flow rate. Due to the unique location of the fan design point being at a higher flow rate than the optimum efficiency point, an objective function that can counter such effects would be desirable. As such, the second objective function considers the slope of the total-to-static efficiency for lower than design point flow rates. This is represented by:

$$
f_{2}(\mathbf{x})=\frac{\eta_{l}-\eta_{D P}^{*}}{\dot{V}_{l}-\dot{V}_{D P}}
$$

where a negative slope value indicates a higher efficiency at lower flow rates. As indicated, this function represents a multi-point objective which ranges over the fan's 
design point flow rate and a lower than design point flow rate. However, due to excessively high transient simulation times reported for all lower than design point flow rates (Section 4.2), only steady state simulations will be considered for the optimization procedure. Consequently, the lowest flow rate of the datum fan meeting the specified convergence criteria (as set out in Section 3.4 .3 ) is $\dot{V}_{l}=13,22 \mathrm{~m}^{3} / \mathrm{s}$.

The final form of the current multi-objective multi-point (MOMP) objective function is represented by the weighted sum of objectives (as explained in Section 2.6.2). As both objectives are of the same order of magnitude, weightings are set to 1 (likewise with that of Deng et al. (2013) who used a similar objective function for the design optimization of a transonic fan blade). The final objective function used in this investigation is represented by:

$$
F(\mathbf{x})=1 \times\left(\frac{\eta_{D P}-\eta_{D P}^{*}}{\eta_{D P}}\right)+1 \times\left(\frac{\eta_{l}-\eta_{D P}^{*}}{\dot{V}_{l}-\dot{V}_{D P}}\right)+\sum_{m=1}^{M} w_{m} p_{m}(\mathbf{x}) .
$$

where:

$\sum_{m=1}^{M} w_{m} p_{m}(\mathbf{x})$ represents the modification of the objective function to include the inequality constraints.

\subsubsection{Boundary constraints}

The selected TE end-plate design is described by three predominant dimensions, namely:

1. Length $\left(l_{e p}\right)$

2. Height $\left(h_{e p}\right)$, and

3. End-plate setting angle $\left(\zeta_{e p}\right)$.

These represent the input control variables (dimensionality $k=3$ ) that will be used to influence the response value of the optimization routine. In order to be able to fully describe the possible design space, the control variable bounds need to first be selected. Due to the novelty of the current design, little is known about the effect of the aforementioned control variables on the fan's performance characteristics. As a result of these uncertainties, a method similar to that of a full factorial design will be used to ascertain the relative importance of each control variable on the solution in order to better estimate an appropriate value for their bounds. 
A two-level full factorial design method compares the response values of all possible combinations of the upper $(+)$ and lower $(-)$ bounds of the control variables $\left(X_{i}\right)$ to be able to determine the relative importance of each on the solution's result. Each level is represented by $2^{k}$ different designs, as illustrated in Figure 7.1 .

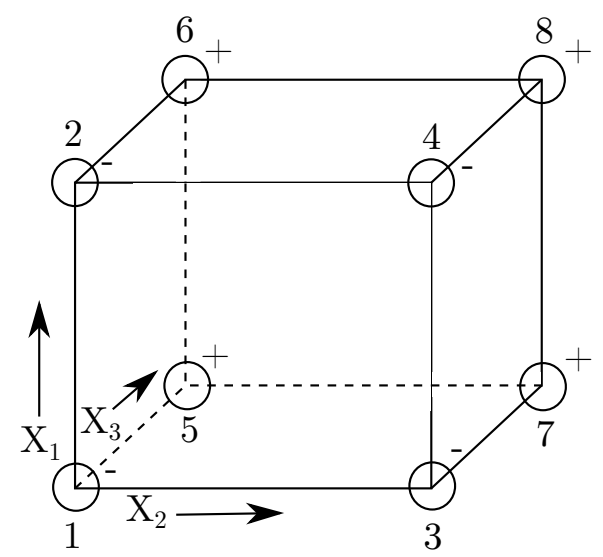

Figure 7.1: Two-level, full factorial design $\left(k=3\right.$ and $\left.2^{k}=8\right)$

The initial bounds used in the full factorial design of the TE end-plate will be based on the preliminary study conducted in Chapter 6. In order to prevent extraneous factors having an affect on the results (such as separation caused by a high end-plate setting angle or infeasible designs due to poorly chosen lower bounds), the control variables will be randomized between an upper $[0.66,1.0]$ and lower [0.0, 0.33] range rather than a single bound. The numerical results of the full factorial TE end-plate design are illustrated in Figure 7.2, with all values non-dimensionalized with respect to the maximum value of each respective independent variable. 


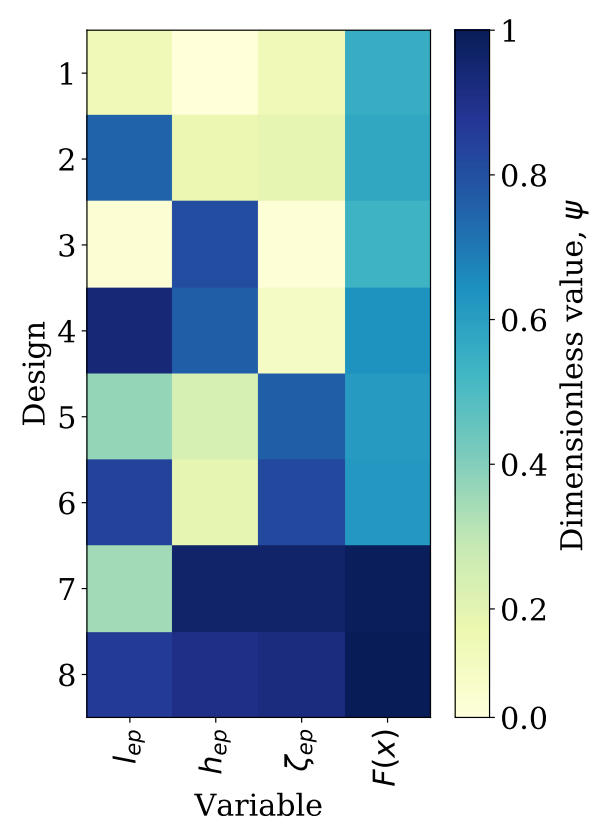

Figure 7.2: Two-level, full factorial design of TE end-plate design

As evident by the end-plate's objective function $F(x)$ (Section 7.2.1), larger control variable values are synonymous with an increase in the end-plate's response value. However, when comparing design 7 and 8, it becomes apparent that the end-plate's length $l_{e p}$ has a lesser effect on the overall result. From this it can be concluded that the $h_{e p}$ and $\zeta_{e p}$ parameters have the biggest influence on the response value of the TE end-plate design. Consequently, the upper bounds of these variables will be chosen based on the maximum AoA of a similar sized flat plate (Figure 6.9) and the maximum end-plate height which is still able to follow the curvature of the blade's TE without having to introduce more variables into the design (i.e. using splines). Additionally, a conservative value will nonetheless be chosen for the upper bound on the end-plate's length. Table 7.1 summarizes the chosen upper and lower bounds of the input control variables of the TE end-plate design.

Table 7.1: Control variable bounds

\begin{tabular}{ccc}
\hline Control variable & LB & UB \\
\hline$l_{e p}$ & $0 \mathrm{~mm}$ & $70 \mathrm{~mm}$ \\
$h_{e p}$ & $0 \mathrm{~mm}$ & $100 \mathrm{~mm}$ \\
$\zeta_{e p}$ & $0^{\circ}$ & $40^{\circ}$ \\
\hline
\end{tabular}




\subsubsection{Inequality constraints}

The inequality constraints for the current optimization procedure are likewise aligned with the design considerations set out in Section 6.1. As the objective function already takes the fan's efficiency improvement into account, a total-tostatic pressure inequality is chosen to ensure improvement at the fan's design point (as seen in all preliminary TE end-plate designs). This inequality is represented by:

$$
g_{1}(\mathbf{x})=\frac{\Delta p_{D P}-\Delta p_{D P}^{*}}{\Delta p_{D P}} \leq 0
$$

which is enforced by a quadratic penalty function (as given in Equation 2.10). This value is then incorporated into the final objective function through the modified objective function formula (given in Equation 2.7) to steer non-feasible solutions towards the feasible region.

As outliers are likely to occur during initialization of the design space, extreme prejudice is given to such designs to ensure no adverse effects on the optimization routine. These designs were found to be synonymous with a severely low design point total-to-static efficiency. As such, the other inequality constraint used in this investigation is represented by:

$$
g_{2}(\mathbf{x})=f_{1}(\mathbf{x})=\frac{\eta_{D P}-\eta_{D P}^{*}}{\eta_{D P}} \leq 0
$$

where the bounce-back method (as discussed in Section 2.7.1) is used to enforce constraint conformity. The base vector used in this method is chosen to be the nearest feasible vector to the violating vector based on its Euclidean distance $\left(L_{2}\right.$ norm). The objective function value of the violating vector is then set to be equal to half the value of the feasible base vector. This is done in order to limit any severe changes to the response surface caused by outliers. An example of this method is illustrated in Figure 7.3 . 


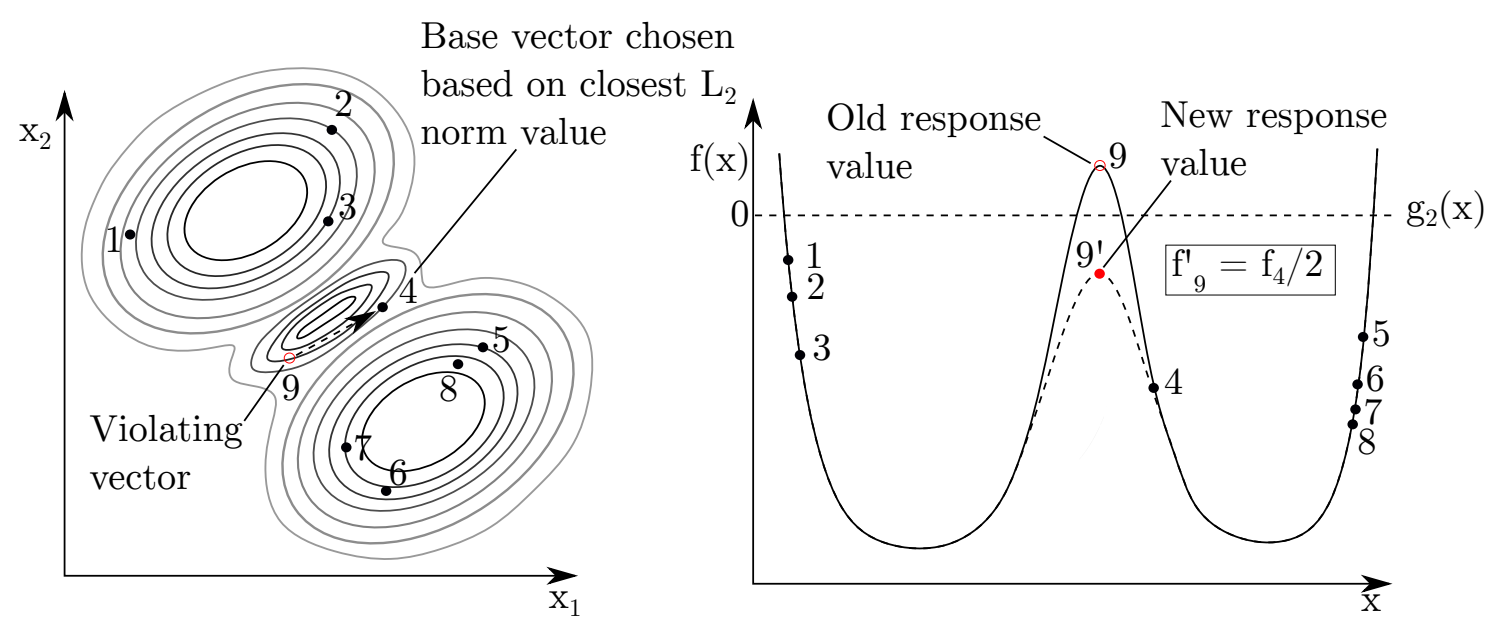

Figure 7.3: Graphical illustration with true (solid) and modified (dashed) 1dimensional response curves of adapted bounce-back resetting scheme for replacing non-feasible response values

\subsection{Chapter summary}

The current chapter deals with the identification of the fundamental quantities used in the current end-plate optimization process. This is necessitated as optimization would be ineffective without proper problem definition. The following points summarize the findings:

- Selection of the most suitable design is crucial as optimization has the potential to be a time intensive process. Comparing the results of the two analyzed end-plate designs from Chapter 6, the TE end-plate design was selected for further development. This was due to its superior aerodynamic performance characteristics when compared to the CT end-plate design. This design was also found to meet the design requirements as specified in Section 6.1.

- A multi-objective multi-point (MOMP) objective function was selected to describe the shape of the optimization algorithm's response surface. Using the weighted sum method, this function incorporates both primary and secondary objectives. The former, being the improvement of the fan's totalto-static efficiency, was chosen as this metric gives the relative correlation between the fan's pressure and power characteristics. The latter objective includes the effect of slope to compensate for adverse wind conditions as these are commonly encountered during the fan's operation. Additionally, various penalty terms are included in the formulation of the objective function in an attempt to steer the algorithm away from non-feasible solutions. 
- Finally, a full factorial design of the TE end-plate's various design parameters is performed in order to determine their influence on the fan's performance characteristics. The results of which are used to determine adequate design space bounds for later exploration during optimization. 


\section{Chapter 8}

\section{End-plate optimization routine}

\subsection{Background}

A typical problem faced by engineers when considering the design optimization of turbomachinery is the desire for increased model accuracy while simultaneously reducing the computational expense associated with that increase in accuracy. As such, the type of surrogate model (SM) chosen is very much situational dependent. However, as noted in Chapter 4 , simulation times of greater than 48 hours are reported for modelling the M-fan during this investigation. This means that using a large number of function evaluations for the current optimization problem becomes impractical. As such, the chosen SM needs to take this into consideration.

Several SMs were considered for the current optimization problem, these included: artificial neural networks (ANNs), radial basis function interpolations (RBFs), and Kriging based Gaussian process models (GPs). In a recent study involving a comparison between the latter two models, Bagheri et al. (2017) found the RBF to perform better overall in terms of its accuracy and computational time. This is due to the GP model requiring some form of regularization in its correlation matrix thereby leading to a less accurate solution. However, one of the big advantages of Kriging based models is that they provide an estimation of their own uncertainty when making predictions. This is worth noting as the algorithm attempts to compromise between searching areas of low model veracity while still exploring areas with a high likelihood of objective function improvement. This further increases the algorithm's likelihood of improving the best known solution.

As a result of this uncertainty information being readily available, the infill search criterion could be used to sequentially reduce the algorithm's search area to areas of higher improvement. This concept has been reported as early as 1978 (Mockus 
et al. 1978) and has more recently been found to have a strong influence on the algorithm's ability to efficiently and accurately locate optima (Sasena et al., 2002). This reduces the need for exhaustive search methods thereby making this method highly attractive as the required number of function evaluations might be reduced. It is for these reasons that the Kriging based model is chosen for use in the current investigation.

A routine that makes use of a derivative of the Kriging based model is that of the Design and Analysis of Computer aided Experiments (DACE) surrogate model of Sacks et al. (1989) with the accompanying Efficient Global Optimization (EGO) algorithm of Jones et al. (1998). This model has shown to be robust in its application while still being able to predict the global optima of even the most deceptive non-linear and multi-modal functions (Forrester et al., 2006). More recently, Bergh (2018) found good agreement with experimental results when employing this method in the design optimization of a single stage turbine rotor. The following sections detail the optimization routine used in this investigation. An overview of the process flow is shown in Section 8.6.

\subsection{Design space population}

Before the optimization process can begin, the DACE surrogate model requires an initial population $(\mathbf{X})$, comprising of a set of control vectors $(\mathbf{x})$ and their corresponding response values $(\mathbf{y})$, within the given bounds of the design space. This is done in order to create an initial database from which the model can be trained before commencing the optimization process. As such, the following sections give details of the sampling method used in this investigation.

\subsubsection{Sampling method}

Historically, traditional design of experiment (DoE) techniques were used in the construction of SMs of computer experiments, with the most common being the so called space-filling designs (Bergh, 2018). The aim of these methods is to obtain an adequate trend in response values of the input domain in the most efficient manner possible (i.e. minimal number of sample points). One of the most popular sequential methods employed for design space-filling is that of Latin Hypercube Sampling (LHS) (Liu et al., 2016).

LHS is achieved by separating each dimension into $n$ equally spaced intervals or strata, then randomly selecting one point that falls in between each interval for each dimension. Each point is then randomly paired with another to form the 
coordinates of the final sample point. According to Crombecq et al. (2011), this construction produces a non-collapsing experimental design which guarantees each sample to be at least $\frac{2}{n} \sqrt{2}$ away from the closest other sample in a $[-1,1]^{k}$ design space.

As noted by both Crombecq et al. (2011) and Bergh (2018), although this method guarantees stratification of all points, the random pairing of the coordinate pairs does not guarantee adequate space-filling properties. This is illustrated in Figure 8.1 where both samples represent valid Latin hypercubes. However, as has been demonstrated by Crombecq et al. (2009), the computational expense associated with the generation of a good space-filling Latin hypercube (within a reasonable amount of time) is significant. As such, verification of the sampling method is required in order to ensure adequate space-filling properties. This is further discussed in Section 8.2.2.

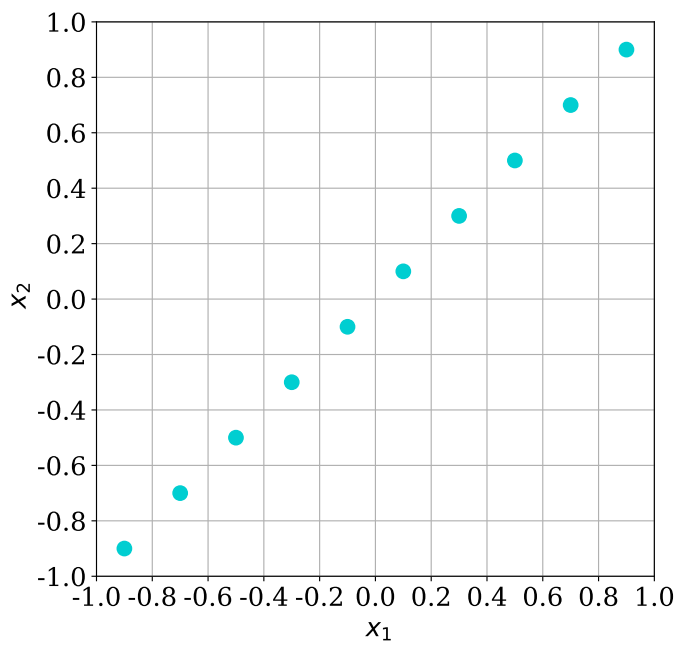

(a) Bad Latin hypercube

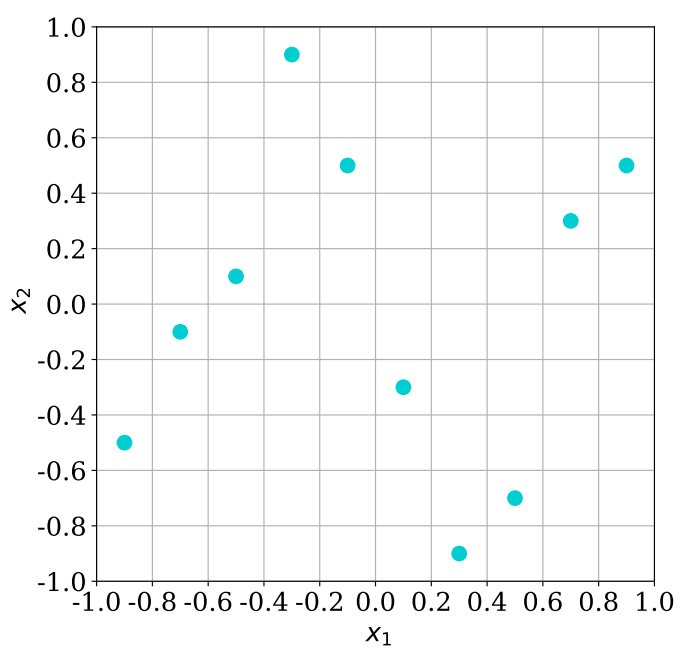

(b) Optimized Latin hypercube

Figure 8.1: Comparison of two different Latin hypercubes

In a comparative study involving Monte Carlo simulation, LHS, maximinLHS, and a multivariate extension of LHS (LHSMDU), Deutsch and Deutsch (2012) found the LHSMDU method to demonstrate significant improvements over the other sampling methods for both low and high dimension cases. This was attributed to a greater degree of multidimensional uniformity. As such, LHSMDU is chosen for implementation in the current investigation. 
As the LHSMDU algorithm is derived from the traditional LHS algorithm, its structure is essentially the same. The differences between the algorithms are realized in the way the variables are ordered. Traditional LHS achieves this through uniform random ordering of each variable into $n$ strata based on an equal probability of $1 / n$. LHSMDU builds on top of this by pre-generating uniform random realizations of the $n$ input variables and eliminating close realizations based on their average correlation. Further details into the algorithm's structure can be found by referring to Deutsch and Deutsch (2012).

\subsubsection{Database construction}

As per the recommendation of Jones et al. (1998), a minimum of $n=10 k$ points is suggested for population of the initial DACE database (where $k$ represents the dimensionality of the problem). In this investigation, this rule is slightly deviated from in order to improve the inter-point spacing between each variable range and to additionally compensate for non-physical designs (as a result of low values for $l_{e p}$ and $\left.h_{e p}\right)$. As such, a total of 35 sample points is chosen for the initial DACE database of the current three dimensional problem.

Once constructed, the 2-dimensional projections between each pair of control variables are checked to ensure an even distribution of points (as seen in Figure $8.2 \mathrm{a}$ through c). Once found to be satisfactory, all non-feasible designs are removed from the database (a total of three non-feasible designs were found, resulting in the final database consisting of 32 sample points). The remaining feasible designs are meshed and simulated using the aforementioned numerical modelling strategy presented in Chapter 3 . 


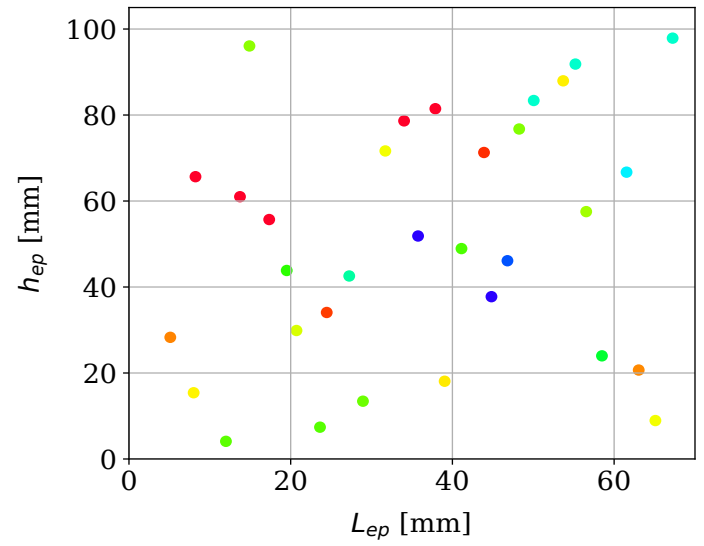

a) $L_{e p}-h_{e p}$ projection plane

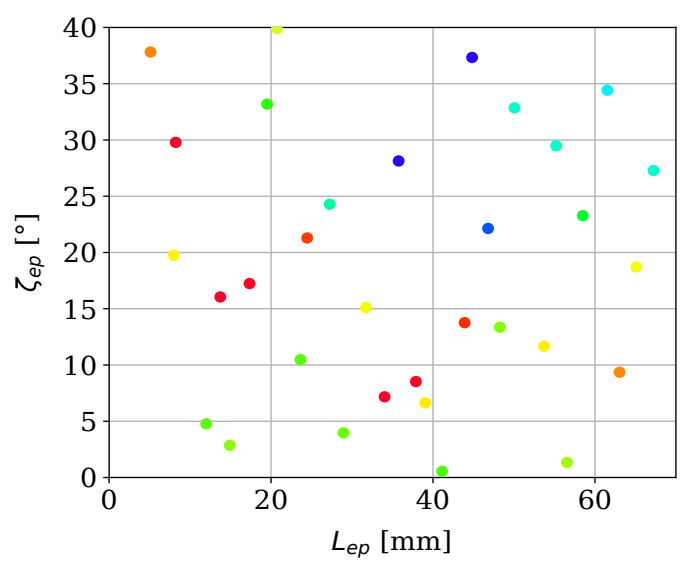

c) $L_{e p}-\xi_{e p}$ projection plane

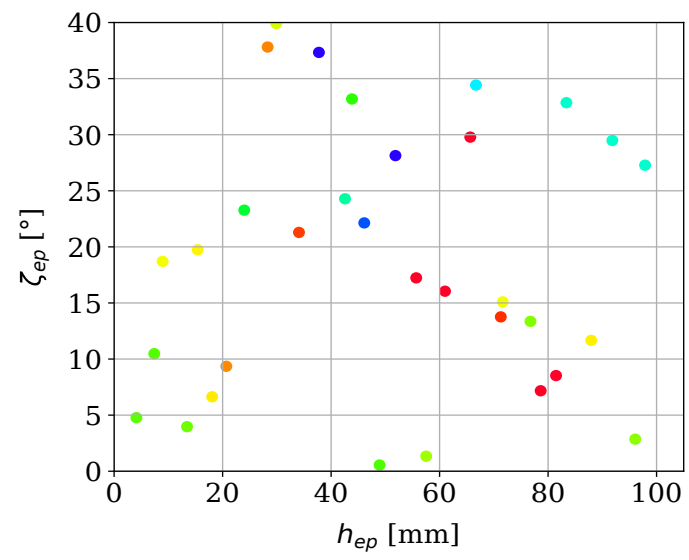

b) $h_{e p}-\xi_{e p}$ projection plane

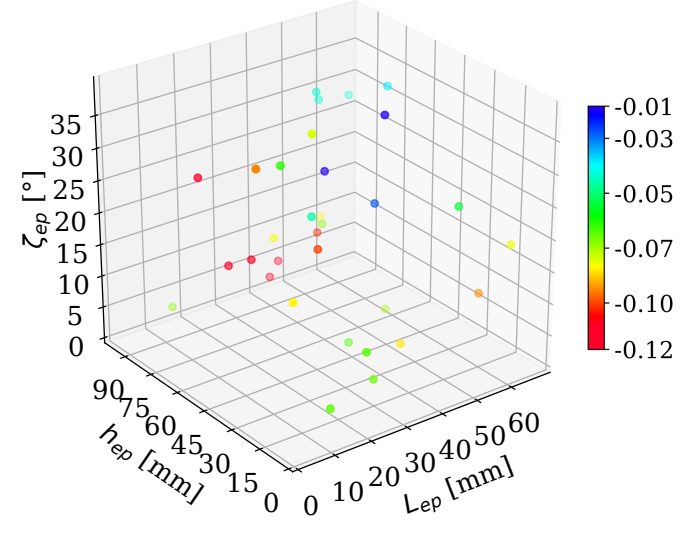

d) Initial $\mathbf{X}_{\text {database }}$

Figure 8.2: Initial DACE database using LHSMDU method for TE end-plate design

As noted by Bergh (2018), the removal of any data point from the initial database means that it can no longer be considered a true Latin hypercube. As previously mentioned, additional data points were added above the minimum required number of points to compensate for this fact, therefore any differences are assumed to be negligible. Nonetheless, the database is later validated in Section 9.1 to ensure the error is acceptable. 


\subsection{The DACE surrogate model}

\subsubsection{Overview}

Similar to the Kriging model, the DACE surrogate model makes use of linear regression to fit a response surface to a predefined set of $n$ data points of dimensionality $k$, as shown in the following equation:

$$
y\left(\mathbf{x}^{i}\right)=\sum_{h} \underbrace{\beta_{h} f_{h}\left(\mathbf{x}^{i}\right)}_{\text {trend model }}+\underbrace{\epsilon\left(\mathbf{x}^{i}\right)}_{\text {deviation }}
$$

where:

$y\left(\mathbf{x}^{i}\right)$ is the associated function value at point $i$, for $(i=1, \ldots, n)$,

$\mathbf{x}^{i}=\left(\mathbf{x}_{1}^{i}, \ldots, \mathbf{x}_{k}^{i}\right)$ is the vector of sample point $i$ with $k$ dimensions,

$\beta_{h}$ is an unknown trend coefficient,

$f_{h}\left(\mathbf{x}^{i}\right)$ is a linear or nonlinear function of $\mathbf{x}$,

$\epsilon\left(\mathbf{x}^{i}\right)$ are normally distributed, independent error terms with $N\left(0, \sigma^{2}\right)$.

Inspection of the model reveals that it is stochastic in nature. Referring to the first term in Equation 8.1, the underlining process trend is modelled through the estimation of the unknown coefficients $\beta_{h}$ of a linear or nonlinear function $f_{h}(\mathbf{x})$. The second term represents stochastic or random deviations from the trend which are modelled as normally distributed independent error terms.

As noted by Jones et al. (1998):

- If the function is indeed deterministic, the error terms can be thought of as essentially collections of left-out terms in $\mathbf{x}$ as any lack of fit would be entirely a result of modeling error and not measurement error.

- Moreover, if the objective function is continuous, then $\epsilon(\mathbf{x})$ will also be continuous. If this is the case, and taking two samples $\left(\mathbf{x}^{*}\right.$ and $\left.\mathbf{x}^{i}\right)$ that are close together as in Figure $8.3 \mathrm{~b}$, then it is reasonable to assume that their respective deviations $\left(\epsilon\left(\mathbf{x}^{*}\right)\right.$ and $\left.\epsilon\left(\mathbf{x}^{i}\right)\right)$ should also be related to each other or "correlated" in some way. 


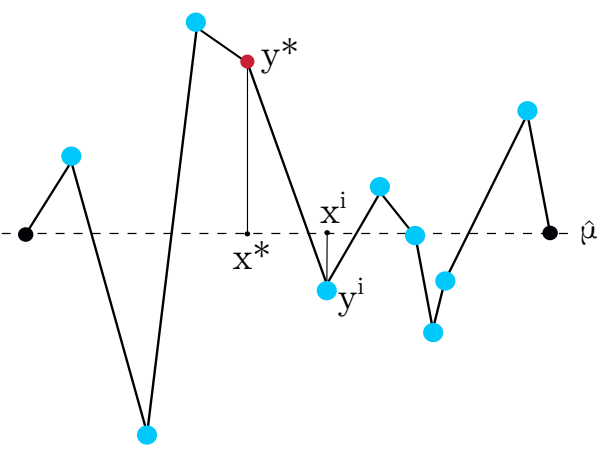

a) Uncorrelated stochastic process

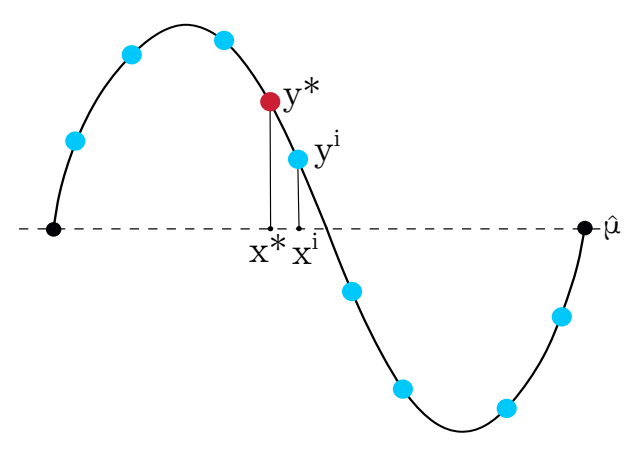

b) Correlated stochastic process

Figure 8.3: The DACE surrogate model concept - the differences in prediction between (a) an uncorrelated process and (b) a correlated process (adapted from Bergh (2018)). As the prediction at $x^{*}$ is close to that of $x^{i}$ in (b), it can be adjusted to take into account for the positive residual at $x^{i}$. Conversely, there is no correlation in (a).

It is this correlation of the error terms that sets the DACE model apart from other surrogate based models. Many surrogate models assume the functional form of the underlining process trend and expend a great deal of effort in estimating the functions many $\beta_{m}$ coefficients. This not only requires many function evaluations in estimating these parameters but is also usually less accurate in modelling complex responses as the functional form may be erroneously selected (Liu et al., 2016). Instead, the DACE model directs its focus on the accurate estimation of the process trend errors. This means that knowing the functional form of the regression terms a priori is not required, which would in any case make the use of such an elaborate modelling technique superfluous. It is for this reason that this method is particularly attractive for modelling complex and deterministic systems which also provides further motivation for its use in the current investigation.

\subsubsection{Error correlation}

The error terms in the DACE model are correlated through the use of a special weighted distance formula, as shown in Equation 8.2 .

$$
d\left(\mathbf{x}^{i}, \mathbf{x}^{j}\right)=\sum_{h=1}^{k} \theta_{h}\left|x_{h}^{i}-x_{h}^{j}\right|^{p_{h}}
$$

where: 
$\mathbf{x}^{i}, \mathbf{x}^{j}$ are the $i^{\text {th }}$ and $j^{\text {th }}$ input vectors of dimension $k$ (i.e. number of control variables),

$\theta_{h}$ is a weighting parameter applied to the $h^{\text {th }}$ component vector difference, for $(h=1, \ldots, k)$ and $\theta_{h} \geq 0$,

$x_{h}^{i}, x_{h}^{j}$ are the $h^{\text {th }}$ component of the $i^{\text {th }}$ and $j^{\text {th }}$ input vector, respectively,

$p_{h}$ is a smoothness parameter with $p_{h} \in[1,2]$.

As discussed by Jones et al. (1998), this way of calculating the component distance is preferred over the simpler Euclidean distance $\left(L_{2}\right.$ norm) formula as it allows for the individual weighting of the variables as opposed to a uniform one. This allows greater control in manipulating the individual vector components and can subsequently be used to measure their importance or "activity".

Using Equation 8.2, the correlation between the errors at $\mathbf{x}^{i}$ and $\mathbf{x}^{j}$ is determined by:

$$
\operatorname{Corr}\left[\epsilon\left(\mathbf{x}^{i}\right), \epsilon\left(\mathbf{x}^{j}\right)\right]=\exp \left[-d\left(\mathbf{x}^{i}, \mathbf{x}^{j}\right)\right]
$$

The use of Equations 8.2 and 8.3 in describing the correlation function (commonly referred to as a spatial correlation function ( $\mathrm{SCF})$ ) offer some useful properties. Intuitively, it makes sense that two points within the sample space, located relatively far apart, should imply a low correlation; whereas the converse should imply a high correlation. As illustrated Figure 8.4, this is indeed found to be the case when considering the current SCF.

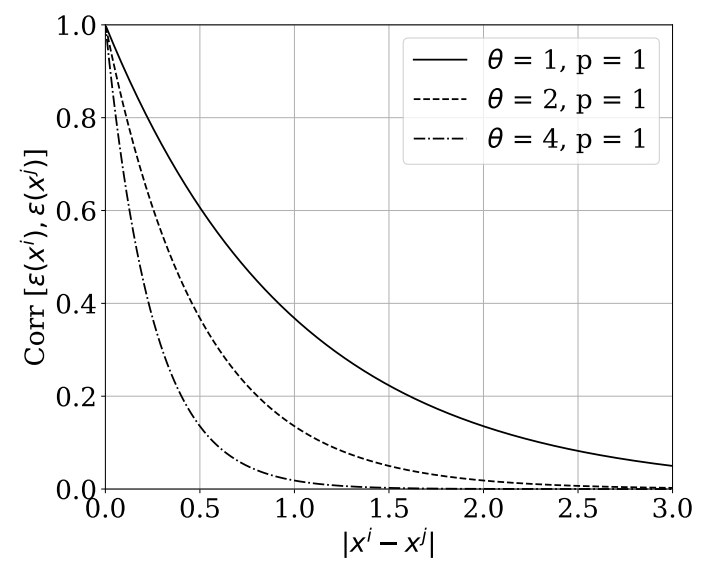

(a)

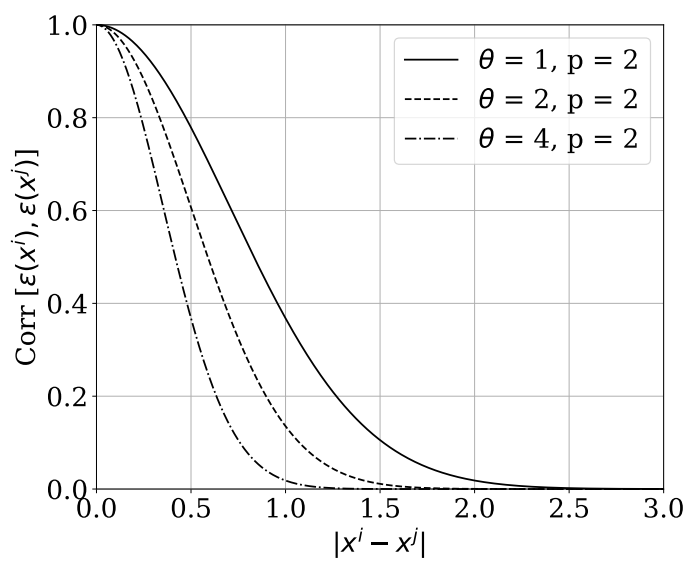

(b)

Figure 8.4: Influence of DACE parameters on correlation function 
As evident in the figure, large values of the sum of the component differences $\left|x_{h}^{i}-x_{h}^{j}\right|$ (i.e. large spatial distances between points) relate to a low correlation; whereas the opposite is also found true. Comparing the various activity levels in each of the two sub-figures, it can be seen that the more active curve $(\theta=4)$ is represented by a steeper change in the correlation value with a change in spatial distance. This effectively means a decrease in the correlation range of the function thereby allowing small differences in spatial distances to have a larger effect on the model. This is important to note as it means that all variables are given an equal opportunity to influence the solution despite having some level of non-homogeneity.

As previously mentioned, the $\mathbf{p}$ exponent is related to the smoothness of the function. This can be seen by comparing Figure $8.4 \mathrm{a}$ to $8.4 \mathrm{~b}$. Considering the relevant literature, the most commonly employed SCF is that of the Gaussian correlation function due to its relatively smooth and infinitely differentiable surface features, making it beneficial for many gradient-based optimization algorithms (Song et al. 2013). This equates to using a value of $p_{h}=2$ for $h=1, \ldots, k$, which is consequently chosen for this investigation.

\subsubsection{Parameter estimation}

The DACE model requires the estimation of $2 k+2$ parameters, namely: $\mu, \sigma^{2}$ and the hyperparameters $\theta_{1}, \ldots, \theta_{k}$, and $p_{1}, \ldots, p_{k}$. The estimation of each is described in the following sections.

\section{Maximum likelihood estimation}

As first introduced by Sacks et al. (1989), the DACE model primarily makes use of the statistics-based method of Maximum Likelihood Estimation (MLE) for estimating the unknown model hyperparameters. This method is preferred as it does not depend on the dimension of the input space (Durantin et al., 2016). The general form of the likelihood function is described by:

$$
\mathcal{L}\left(\boldsymbol{\beta}, \sigma^{2}, \boldsymbol{\theta}, \mathbf{p}\right)=\frac{1}{(2 \pi)^{n / 2}\left(\sigma^{2}\right)^{n / 2} \operatorname{det}(\mathbf{R})^{\frac{1}{2}}} \exp \left[-\frac{(\mathbf{y}-\mathbf{F} \boldsymbol{\beta})^{T} \mathbf{R}^{-1}(\mathbf{y}-\mathbf{F} \boldsymbol{\beta})}{2 \sigma^{2}}\right]
$$

where:

$n$ is the number of sample points,

$\sigma^{2}$ represents the variance of the process errors, 
$\mathbf{R}$ denotes the $n \times n$ correlation matrix whose $(i, j)$ entries correspond to $\operatorname{Corr}\left[\epsilon\left(\mathbf{x}^{i}\right), \epsilon\left(\mathbf{x}^{j}\right)\right]$

$\mathbf{y}=\left(y^{1}, \ldots, y^{n}\right)^{T}$ are the observed objective function values,

$\mathbf{F}$ denotes a matrix whose $(i, k)$ entries correspond to $f_{h}\left(\mathbf{x}^{i}\right)$,

$\boldsymbol{\beta}=\left(\beta_{1}, \ldots, \beta_{k}\right)^{T}$ denotes a $n$ vector of trend coefficients.

Given four input parameters, two of which are estimated hyperparameters $\boldsymbol{\theta}$ and $\mathbf{p}$, the only remaining values required to solve become $\boldsymbol{\beta}$ and $\sigma^{2}$. By taking the derivative of Equation 8.4 with respect to $\boldsymbol{\beta}$ and $\sigma^{2}$ and solving for zero, the parameter values that maximize the likelihood function in closed form are realized with:

$$
\begin{gathered}
\hat{\boldsymbol{\beta}}=\frac{\mathbf{F}^{T} \mathbf{R}^{-1} \mathbf{y}}{\mathbf{F}^{T} \mathbf{R}^{-1} \mathbf{F}} \\
\hat{\sigma^{2}}=\frac{(\mathbf{y}-\mathbf{F} \boldsymbol{\beta})^{T} \mathbf{R}^{-1}(\mathbf{y}-\mathbf{F} \boldsymbol{\beta})}{n}
\end{gathered}
$$

Through substitution of Equations 8.5 and 8.6 into the likelihood function, Jones et al. (1998) express the result as a "concentrated likelihood function" $\left(\mathcal{L}_{c}\right)$ which depends only on the parameters $\boldsymbol{\theta}$ and $\mathbf{p}$ for $h=1, \ldots, k$. As the computational effort required increases with the number of model parameters, the concentrated likelihood function is often superseded by the simpler log-likelihood profile as the number of model parameters that require solving are reduced. As indicated by Everitt (2012), maximization of the latter leads to the same estimates as that of the former. Moreover, as the SCF is known a priori, the log-likelihood profile becomes a function of $\boldsymbol{\theta}$ only, thereby reducing the overall number of hyperparameters of the DACE model to an equal dimensionality of the problem at hand (i.e. $k$ ). The log-likelihood function is represented by the following equation:

$$
\ln \left(\mathcal{L}_{c}(\boldsymbol{\theta})\right)=-\frac{n}{2} \ln \left(\sigma^{2}\right)-\frac{1}{2} \ln (\operatorname{det}(\mathbf{R}))
$$

As noted by Martin and Simpson (2004), no closed-form solution for the optimal hyperparameters of the most common SCFs exists. This is often a result of the unattainable explicit solving of the twice differential of the likelihood function $\left(\frac{\partial \mathcal{L}_{c}}{\partial \theta_{h}}=0\right.$ and $\left.\frac{\partial^{2} \mathcal{L}_{c}}{\partial \theta_{h}^{2}}<0\right)$ thereby necessitating iterative optimization algorithms in order to solve (Everitt, 2012). As typical numerical optimization techniques predominately involve the minimization of the objective function, the current loglikelihood function is reformulated to represent that of a minimization problem: 


$$
\underset{\theta_{h} \in \mathbb{R}^{k}}{\operatorname{minimize}}:-\ln \left(\mathcal{L}_{c}(\boldsymbol{\theta})\right)
$$

subject to

$$
\theta_{h}>0
$$

with

$$
h=1, \ldots, k
$$

It should be noted at this point that the MLE function formulate above still contains a matrix of unknown linear or non-linear functions of $\mathbf{x}$, namely $\mathbf{F}$. This, combined with the $\boldsymbol{\beta}$ terms, models the trend of the DACE model. The following section describes the modelling of these trend parameters.

\section{Trend modelling}

When considering trend modelling, reference to the Kriging method must again be made here. Various variants of the Kriging method exist within literature, all of which are similar in their means of modelling the process trend. If the trend is known a priori (i.e. all parameters and covariates are completely described), a constant is used to model the process trend. This method is referred to as Simple Kriging and assumes that the trend is exactly known and represented by a fixed constant value. Figure 8.5 illustrates an example of this method with the solid circles representing the observed data.

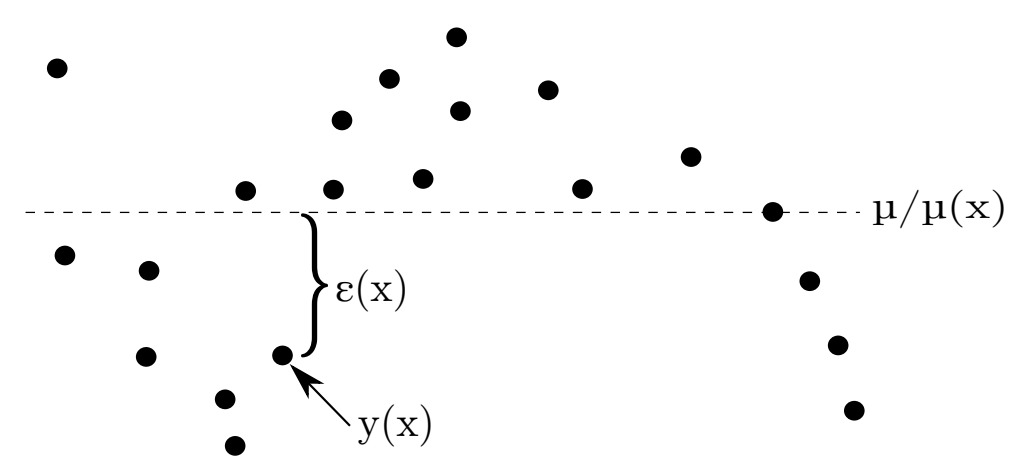

Figure 8.5: Simple and Ordinary Kriging example

Depending on the application, this method can be unrealistic in that it may often be impractical or even impossible to be able to completely define the process trend before hand. Furthermore, the assumption of a fixed trend may be erroneous if new sampled data is introduced into an inadequately resolved model which may alter the trends characteristics. An adaptation of this method that takes these 
limitations into consideration is known as Ordinary Kriging. Given an unknown process trend, this method determines the constant by which the trend is modelled during the fitting of the model. This means that the value of the constant fluctuates with the mean of the trend and is thus better able to incorporate new data.

The final method discussed is that of Universal Kriging. As shown in Figure 8.6. this method makes use of a general polynomial regression to model the trend which is then fit to the data such that the mean of all process errors $\epsilon(x)$ is zero.

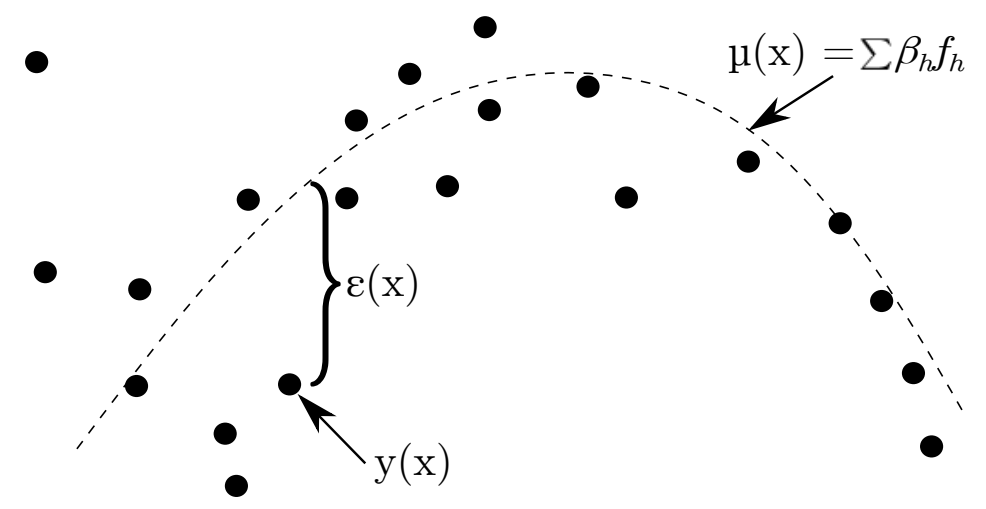

Figure 8.6: Universal Kriging example

In a study involving various complexities of trend functions used in the Kriging model, Martin and Simpson (2004) found that increasing the model's complexity provided for (in most cases) a better approximation of a deterministic computer model. This increase in complexity appeared to also improve the shape of the log-likelihood function thereby making it easier to determine its global maximum. This would make the determination of the model hyperparameters in the MLE approach easier as the models complexity is reduced. Table 8.1 summarizes the discussed trend models.

Table 8.1: Kriging model trend functions

\begin{tabular}{ccc}
\hline Variant & Trend model & Characteristic \\
\hline Simple & $\mu$ & known, constant \\
Ordinary & $\mu(x)$ & unknown, constant \\
Universal & $\sum_{h} \beta_{h} f_{h}$ & unknown, polynomial \\
\hline
\end{tabular}


The DACE model makes use of Ordinary Kriging in modelling its process trend. In this form, the influence of the unknown linear or non-linear function matrix $\mathbf{F}$ is negated by substitution of a $n \times 1$ vector of ones such that $\mathbf{F} \equiv \mathbf{1}=\left(1^{1}, \ldots, 1^{n}\right)^{T}$. The $\boldsymbol{\beta}$ trend coefficients are modelled by a constant $\hat{\mu}$ term which varies with x. By substituting this into the generalized form in Equations 8.5 and 8.6, the reformulated parameter values that maximize the likelihood function in closed form are given by:

$$
\begin{gathered}
\hat{\mu}=\frac{\mathbf{1}^{T} \mathbf{R}^{-1} \mathbf{y}}{\mathbf{1}^{T} \mathbf{R}^{-1} \mathbf{1}} \\
\hat{\sigma^{2}}=\frac{(\mathbf{y}-\mathbf{1} \hat{\mu})^{T} \mathbf{R}^{-1}(\mathbf{y}-\mathbf{1} \hat{\mu})}{n}
\end{gathered}
$$

where:

$\mathbf{1}=\left(1^{1}, \ldots, 1^{n}\right)^{T}$ denotes a $n \times 1$ vector of ones,

$\mathbf{R}$ denotes the correlation matrix,

with $\mathbf{y}$ and $n$ as defined previously.

\subsubsection{The DACE predictor}

As previously stated, the DACE model makes use of Ordinary Kriging to model the process trend. Given a mean of the stochastic process, $\mu\left(\mathbf{x}^{i}\right)$, and the process errors, $\epsilon\left(\mathbf{x}^{i}\right)$, the general form of the linear predictor of $y\left(\mathbf{x}^{i}\right)$ is given by:

$$
y\left(\mathbf{x}^{i}\right)=\mu\left(\mathbf{x}^{i}\right)+\epsilon\left(\mathbf{x}^{i}\right)
$$

If $\mathbf{R}$ represents the correlation matrix between the error terms at known data point locations, then a matrix $\mathbf{r}$ with $r_{i}\left(\mathbf{x}^{*}\right) \equiv \operatorname{Corr}\left[\epsilon\left(\mathbf{x}^{*}\right), \epsilon\left(\mathbf{x}^{i}\right)\right]$ can equally be defined to represent the correlation between the error terms at an unknown location $\mathbf{x}^{*}$ and the known locations $\mathbf{x}^{i}$ (using Equations 8.2 and 8.3 as defined previously). Then, by reformulating Equation 8.11, the best linear unbiased predictor of $y\left(\mathbf{x}^{*}\right)$ (as derived by Sacks et al. (1989)) can be written as

$$
\hat{y}\left(\mathbf{x}^{*}\right)=\hat{\mu}+\mathbf{r}^{T} \mathbf{R}^{-1}(\mathbf{y}-\mathbf{1} \hat{\mu})
$$

where:

$\mathbf{r}=\left(r^{1}, \ldots, r^{n}\right)^{T}$ denotes an $n$-vector of correlations whose $i^{t h}$ entry corresponds to $\operatorname{Corr}\left[\epsilon\left(\mathbf{x}^{*}\right), \epsilon\left(\mathbf{x}^{i}\right)\right]$,

with $\hat{\mu}, \mathbf{R}, \mathbf{y}$ and $\mathbf{1}$ as defined previously. 


\subsection{Model error and validation}

Due to the DACE model providing estimates based on an initial discrete set of sampled data points, uncertainties in its predictions are an inevitable consequence as the black-box type function may not adequately be described by the model. Therefore, this section describes the validation procedure of the model to ensure that these uncertainties are sufficiently minimized.

\subsubsection{Model error}

As previously mentioned, one of the main advantages of the Kriging based models is that they provide an estimation of their own uncertainty in their predictions. As noted by Jones et al. (1998), the correlation between the error terms should also affect the estimate of the models prediction accuracy. With this, a mean squared error of the DACE predictor can be defined as

$$
s^{2}\left(\mathbf{x}^{*}\right)=\sigma^{2}\left[1-\mathbf{r}^{T} \mathbf{R}^{-1} \mathbf{r}+\frac{\left(1-\mathbf{1}^{T} \mathbf{R}^{-1} \mathbf{r}\right)^{2}}{\mathbf{1}^{T} \mathbf{R}^{-1} \mathbf{1}}\right]
$$

The second term on the right hand side of Equation $8.13, \mathbf{r}^{T} \mathbf{R}^{-1} \mathbf{r}$, represents the reduction in the prediction error based on the correlation of the prediction point $\mathbf{x}^{*}$ with that of the sampled points in $\mathbf{x}^{i}$. The $\left(1-\mathbf{1}^{T} \mathbf{R}^{-1} \mathbf{r}\right)^{2} / \mathbf{1}^{T} \mathbf{R}^{-1} \mathbf{1}$ term represents the uncertainty in estimating the trend mean $\mu$ as the precise value is not known a priori. If $\mathbf{r} \sim \mathbf{0}$ (i.e. indicating no correlation), both the prediction and trend mean adjustment would also tend to zero (i.e. $\mathbf{r}^{T} \mathbf{R}^{-1} \mathbf{r} \sim 0$ and $\mathbf{1}^{T} \mathbf{R}^{-1} \mathbf{r} \sim 0$ ) with the error term tending roughly to the same value as that of the models variance (i.e. $\left.s^{2}\left(\mathbf{x}^{*}\right) \rightarrow \sigma^{2}\right)$.

In the converse case (i.e. complete correlation with $\mathbf{r} \sim \mathbf{1}$ ), the models error would tend to zero (i.e. $s^{2}\left(\mathbf{x}^{*}\right) \rightarrow 0$ ). This makes intuitive sense as if $\mathbf{x}^{*}$ is relatively close to any of the other points in $\mathbf{x}^{i}$, its variance (and subsequent error) should also be relatively low which would relate to a high confidence in the prediction of $\mathbf{x}^{*}$. As noted by Jones et al. (1998), it is often more convenient to take the root of the mean square error, $s=\sqrt{s^{2}(\mathbf{x})}$, to give the $R M S E$ for measuring the uncertainty in the models predictions.

\subsubsection{Cross-validation}

Being able to define the DACE model's error based on its own uncertainty allows for an attractive model-validation test by which the satisfactory fit of the model's hyperparameters are determined. One of the most common methods employed to cross-validate the DACE model is that of the leave-one-out (LOO) analysis (Bergh. 
2018). This method iteratively removes a sample point from the database by which it then fits the model and makes predictions to estimate the removed sample's value using the reduced database. The estimated and removed values are then compared to determine satisfactory model fit and hyperparameter estimation.

The steps used to cross-validate the DACE model are summarized as follows:

1. Given a sample database $\mathbf{x}^{i}$, fit the DACE model to the database and compute the optimized hyperparameter $\boldsymbol{\theta}$,

2. Remove the $i^{\text {th }}$ observation from the database to give a reduced database of $n-1$ points, denoting the removed observation's response value by $y\left(\mathbf{x}^{i}\right)$,

3. Using the $n-1$ points in the reduced database, reformulate the DACE parameters $\mathbf{r}_{-i}$ and $\mathbf{R}_{-i}$ in Equation 8.12 using the hyperparameter $\boldsymbol{\theta}$ (previously estimated using all the sampled observations),

4. With these reduced parameters, estimate the prediction $\hat{y}_{-i}\left(\mathbf{x}^{i}\right)$ and $R M S E$ $s_{-i}\left(\mathbf{x}^{i}\right)$ and compare this prediction with that of the known value at $y\left(\mathbf{x}^{i}\right)$,

5. Repeat steps 2-4 for all $n$ sampled points in the database.

In practice, validation of the model is conducted by means of three diagnostic tests, namely: 1) actual vs predicted response value comparisons, 2) standardized residual error, and 3) Q-Q plots. The first simply compares the actual value of the removed sample $\left(y\left(\mathbf{x}^{i}\right)\right)$ to the predicted value from the reduced database $\left(\hat{y}_{-i}\left(\mathbf{x}^{i}\right)\right)$. In order for the model to be considered satisfactory, all of the points should lie close to a $45^{\circ}$ dividing line between the two linear axes of the plot which indicates a perfectly fit model.

Ordinarily, the second test would compare the confidence intervals of all the predicted points to ensure that they fall within a specified bounds that relate to the accuracy of the model's predictions. Following Jones et al. (1998), Equation 8.14 introduces a more convenient "standardized cross-validated residual" which computes the number of standard errors that the actual response value is above or below the predicted value:

$$
R_{s t d}=\frac{y\left(\mathbf{x}^{i}\right)-\hat{y}_{-i}\left(\mathbf{x}^{i}\right)}{s_{-i}\left(\mathbf{x}^{i}\right)}
$$

Since the DACE model is approximately $99.7 \%$ confident that its predictions $\left(\hat{y}_{-i}\left(\mathbf{x}^{i}\right)\right)$ lie within three standard deviations of the true value, a simple test used to validate this accuracy is to ensure that the residual of each predicted point lies 
within the interval $-3 \leq R_{\text {std }} \leq 3$.

Finally, a random sample of $n$ independent standard normal deviates are plotted against the standardized cross-validated residuals (Q-Q plots). In order to show that the residuals behave like normal deviates, all points should again lie near a $45^{\circ}$ dividing line between the two linear axes of the plot.

Figure $8.7 \mathrm{a}$ through $\mathrm{f}$ illustrates the differences between a well and poorly fit DACE model using the initial CFD database developed for the end-plate design in Section 8.2. Comparing the actual vs predicted response value plots (Figure $8.7 \mathrm{a}$ and b), the well fit model exhibits an even distribution of predicted values around the $45^{\circ}$ dividing line while the poorly fit model does not. The same can be said for the standardized cross-validated residuals in Figure 8.7k, where all of the residuals fall within the interval $[-3,3]$. Conversely, extreme outliers are present for the poorly fit model in Figure $8.7 \mathrm{~d}$ which exhibits a decreasing residual trend with increasing predicted function values. According to Jones et al. (1998), this suggests a systematic bias in the models predictions.

Finally, the effect of the residuals on the standard normal deviations can be seen by comparing Figures 8.7p and f. The well fit model shows good agreement with that of the $45^{\circ}$ dividing line on the Q-Q plot while extreme deviations on the tail ends of the deviates can be seen for the poorly fit model. 


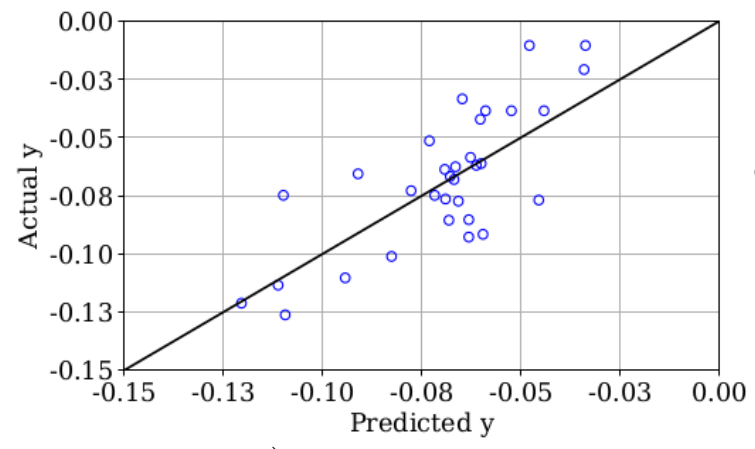

a) $y_{\text {actual }}$ vs $y_{\text {predicted }}$

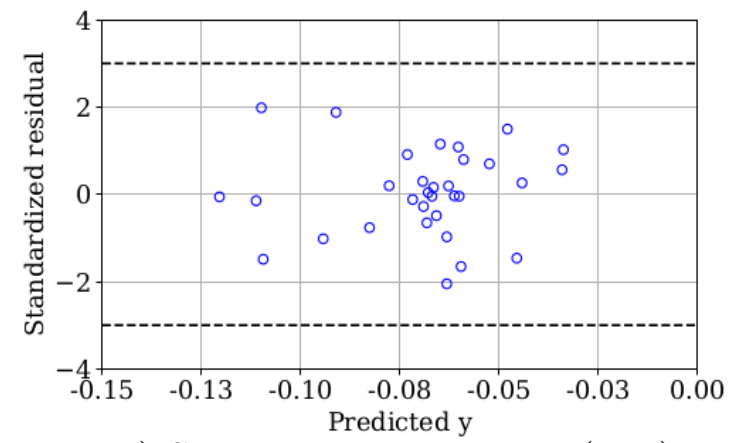

c) Standardized residuals $\left(\mathrm{R}_{\mathrm{std}}\right)$

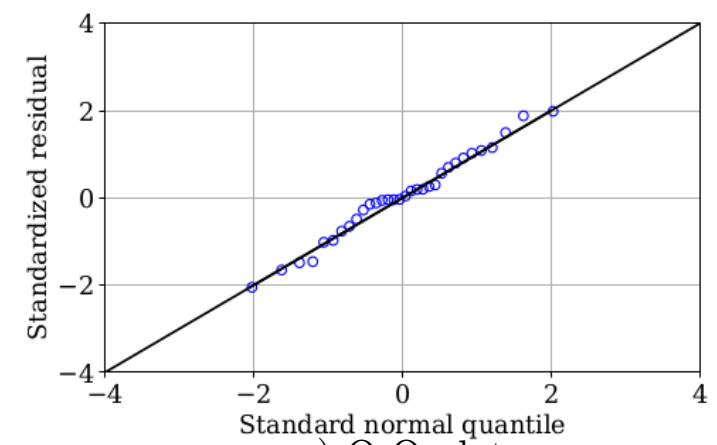

e) Q-Q plot

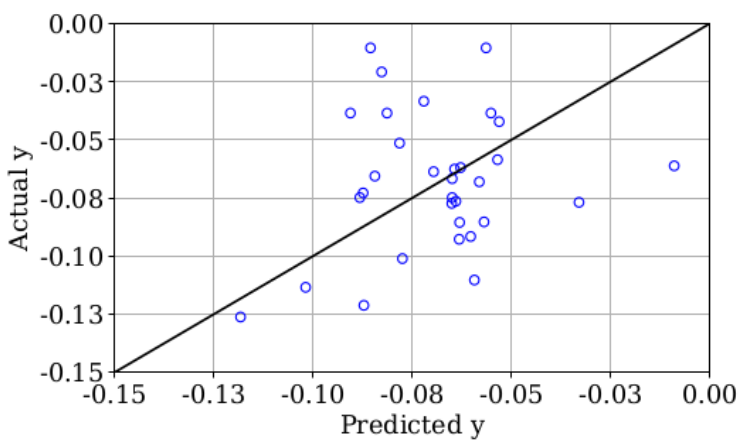

b) $y_{\text {actual }}$ vs $y_{\text {predicted }}$

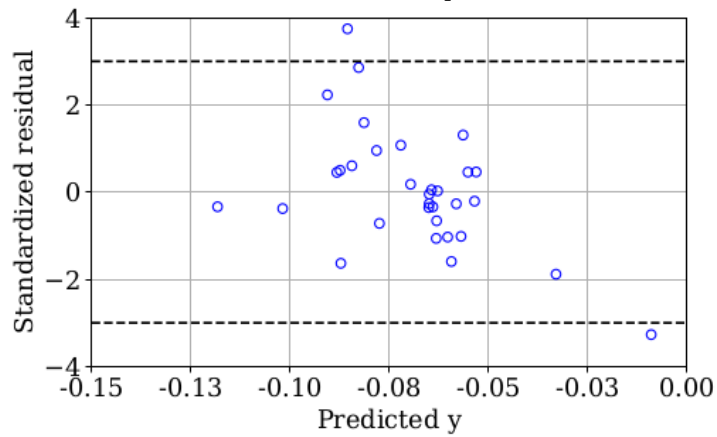

d) Standardized residuals $\left(\mathrm{R}_{\text {std }}\right)$

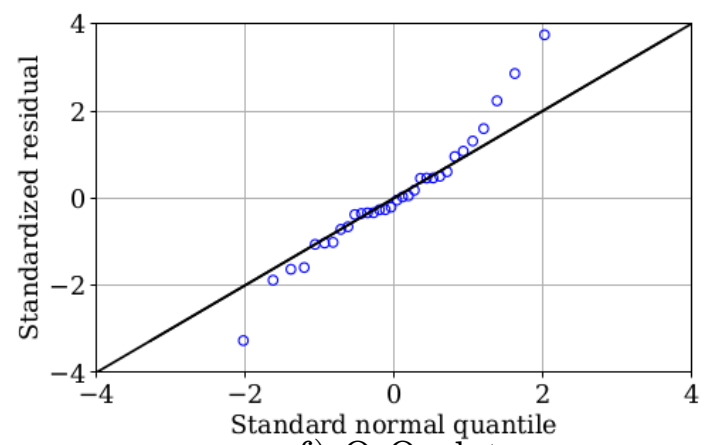

f) Q-Q plot

Figure 8.7: A well (left) and poorly (right) fit DACE model

\subsection{Implementing the DACE model}

Implementation of the DACE model is carried out by first considering some common issues related to the determination of the optimal hyperparameters when fitting the model. According to Bergh (2018), these issues include:

- Increased model complexity when solving the MLE optimization subproblem due to the deceptive multi-modal and non-linear characteristics of the 
concentrated likelihood function (which includes the presence of elongated ridges and flat regions within the functional space),

- Monotonically increasing or decreasing likelihood functions (which have no local optima) with increasing hyperparameter magnitudes as a result of poor modal composition, and

- Rapid degeneration of the correlation matrix $(\mathbf{R})$ condition as a result of decreasing $\boldsymbol{\theta}$ parameter values and repeated sampling of the objective function space.

The following sections discuss the techniques used to address the various outlined issues.

\subsubsection{Optimal hyperparameter estimation}

Various search algorithms are considered for solving the MLE subproblem presented in Section 8.3.3 in order to estimate the optimal hyperparameters when fitting data to the DACE model. Of all the available gradient-based search methods, none are considered for the current investigation due to their likely unsatisfactory performance when locating the global optimum in the context of the current multi-modal and non-linear concentrated likelihood function. This is due to these algorithms being susceptible to becoming stuck along flat portions of the objective function space or reaching premature convergence when encountering local optima (Haupt and Ellen Haupt, 2004).

However, as discussed in Section 2.5.2, one alternative to these types of algorithms are the popular stochastic global optimization algorithms which search for optima in a probabilistic way. These algorithms do not require gradient information nor a potentially biased starting point, thereby negating the previous algorithm's associated disadvantages. As detailed in the same section, the two most common methods trialed for selection in this investigation are:

1. The continuous genetic algorithm (GA) of Haupt and Ellen Haupt (2004) which incorporates elitism and single point crossover, and

2. The rand/1/bin differential evolution (DE) algorithm of Price et al. (2005)

Each algorithm makes use of random re-initialization when modifying out-ofbounds parameters as this method offers the most unbiased approach of the various methods discussed in Section 2.7.1. In order to evaluate both algorithms' performance, each is used to fit the DACE model to the initial TE end-plate database 
constructed in Section 8.2.2. Thereafter, the LOO of Section 8.4 is used to crossvalidate and compare the fitting characteristics of the various models to determine which is most suitable for the current optimization problem. Additionally, the various tuning parameters of each algorithm are trialed between their relevant ranges to determine the optimal settings. The results of the trials are given in Table 8.2

Table 8.2: Comparison of DACE model fitting characteristics with various searching algorithms using the initial TE-end plate CFD database averaged over 5 independent runs

\begin{tabular}{|c|c|c|c|c|c|c|c|c|c|c|}
\hline \multirow[b]{2}{*}{$\mathrm{GA} / \mathrm{DE}$} & \multicolumn{2}{|c|}{$\operatorname{Max} \ln \left(\mathcal{L}_{c}\right)$} & \multicolumn{2}{|c|}{ Max error } & \multicolumn{2}{|c|}{ RMSE } & \multicolumn{2}{|c|}{ Generations } & \multicolumn{2}{|c|}{ Time [s] } \\
\hline & GA & $\mathrm{DE}$ & GA & $\mathrm{DE}$ & GA & $\mathrm{DE}$ & GA & $\mathrm{DE}$ & GA & $\mathrm{DE}$ \\
\hline \multicolumn{11}{|c|}{ Population size } \\
\hline $52 / \underline{50}$ & 121.12 & 121.22 & 2.9659 & 2.9837 & 0.0174 & 0.0174 & 32 & 136 & 74.02 & 180.38 \\
\hline $104 / 100$ & 121.18 & 121.22 & 2.9563 & 2.9837 & 0.0173 & 0.0174 & 50 & 163 & 235.68 & 455.59 \\
\hline $208 / 200$ & 121.22 & 121.22 & 2.9627 & 2.9842 & 0.0173 & 0.0174 & 41 & 102 & 377.22 & 556.62 \\
\hline \multicolumn{11}{|c|}{ Mutation rate } \\
\hline$\underline{0.2} / 0.2$ & 121.22 & 121.20 & 2.9627 & 2.9977 & 0.0173 & 0.0174 & 41 & 148 & 377.22 & 214.28 \\
\hline $0.4 / 0.65$ & 121.10 & 121.22 & 2.7446 & 2.9837 & 0.0172 & 0.0174 & 45 & 177 & 401.63 & 246.97 \\
\hline $0.8 / \underline{0.8}$ & 119.54 & 121.22 & 4.2405 & 2.9800 & 0.0188 & 0.0174 & 26 & 95 & 225.32 & 130.52 \\
\hline \multicolumn{11}{|c|}{ Recombination } \\
\hline$-/ 0.2$ & - & 121.18 & - & 2.8123 & - & 0.0173 & - & 186 & - & 267.64 \\
\hline$-/ 0.5$ & - & 121.21 & - & 2.9850 & - & 0.0174 & - & 86 & - & 118.53 \\
\hline$-/ \underline{0.8}$ & - & 121.22 & - & 2.9783 & - & 0.0174 & - & 89 & - & 126.04 \\
\hline \multicolumn{11}{|c|}{ Max generations } \\
\hline $25 / 50$ & 121.16 & 121.19 & 3.0289 & 3.1011 & 0.0174 & 0.0175 & - & - & 216.28 & 66.44 \\
\hline $100 / 200$ & 121.17 & 121.22 & 2.9518 & 2.9837 & 0.0173 & 0.0174 & - & - & 912.58 & 339.63 \\
\hline
\end{tabular}

A minimum of two tunable parameters are tested for each algorithm, that being population size and mutation rate, with an additional third (recombination rate) being allocated to the DE algorithm. Standard tuning parameter settings are initially selected for each algorithm (as suggest by Haupt and Ellen Haupt (2004) and Price et al. (2005), respectively). Thereafter, each parameter setting is sequentially selected based on the best overall fitting characteristics (as indicated by the underlined parameter values in the first column). It should be noted that $R_{s t d}$ errors are not considered as these values were found to be sufficiently low for all cases and as such met the minimum requirement of $(-3,3)$.

Satisfactory convergence is deemed true once the component-wise vector difference between the current and previous best solution is of the order of $10^{-6}$ for at least 10 consecutive generations once the population's average $\ln \left(\mathcal{L}_{c}\right)$ value is within $1 \%$ of the maximum overall value. This convergence criteria is based largely on personal intuition gained during testing of the various algorithms. As such, the final row in the table represents the results obtained with no convergence criteria but rather a set limit on the maximum number of generations to ensure that the 
suggested convergence criteria is satisfactory.

Considering the results, the GA was found to require a larger population size in comparison to the DE algorithm in order to obtain similar fitting characteristics. Although the GA was found on average to be the more accurate of the two algorithms, this accuracy came at the cost of significantly increased computational time (about 2.73 times greater for the final chosen parameter values). The GA was found to be highly sensitive to inconsistencies during initialization of the population. This occasionally resulted in premature convergence of the algorithm with an unacceptable model error. These findings concur with that found by Ali and Törn (2004), Ali et al. (2005), and Bergh (2018).

Comparison of the final row of tests reveals that both algorithms were found to perform worse when lowering the number of generations. Considering the converse case, no significant change is apparent other than an obvious increase in computational cost. These findings indicate that the suggested convergence criteria is indeed sufficient for use in the current investigation.

Overall, the DE algorithm was found to be the more consistent algorithm, arriving at the same optimal point for each run and at a reduced computational cost when compared to that of the GA. Although slightly less accurate in terms of the model's maximum error and RMSE, the DE algorithm achieved a higher MLE value for all but one of the cases. It is for these reasons stated above that the DE algorithm is selected for use in the current investigation. Based on the current analysis and relevant literature, the final DE algorithm settings are given in Table 8.3.

Table 8.3: Chosen search algorithm parameter settings

\begin{tabular}{cc}
\hline Parameter setting & Value \\
\hline Population size & 50 \\
Mutation rate & 0.8 \\
Recombination rate & 0.8 \\
Min/Max generations & $250 / 1000$ \\
\hline
\end{tabular}

\subsubsection{Hyperparameter initialization and bounds}

A frequent problem associated with solving the MLE subproblem is the potentially poor estimation of the model hyperparameters as a result of poor model composition. According to Kok (2012), this typically occurs when the database consists of very few or poorly distributed sampling points thereby resulting in the 
MLE function decreasing monotonously with either infinitely increasing or zero approaching hyperparameter values. Two remedies are introduced to deal with the above mentioned problems, namely: hyperparameter initialization and hyperparameter bounding.

\section{Hyperparameter initialization}

According to Bergh (2018), initialization of the $\boldsymbol{\theta}$ hyperparameter weights can have a significant effect on the outcome of the maximum likelihood function value. As such, two different coordinate systems are chosen for investigation, namely: linear and logarithmic space. The effect on the concentrated ln-likelihood function for Rosenbrock's 2D valley test function when using the two aforementioned coordinate systems to initialize the $\boldsymbol{\theta}$ parameter space is illustrated in Figures $8.8 \mathrm{a}$ through $d$. The test database consists of 21 points sampled using the LHSMDU method of Section 8.2 over the variable range $\mathbf{x} \in[-30,30]$. 


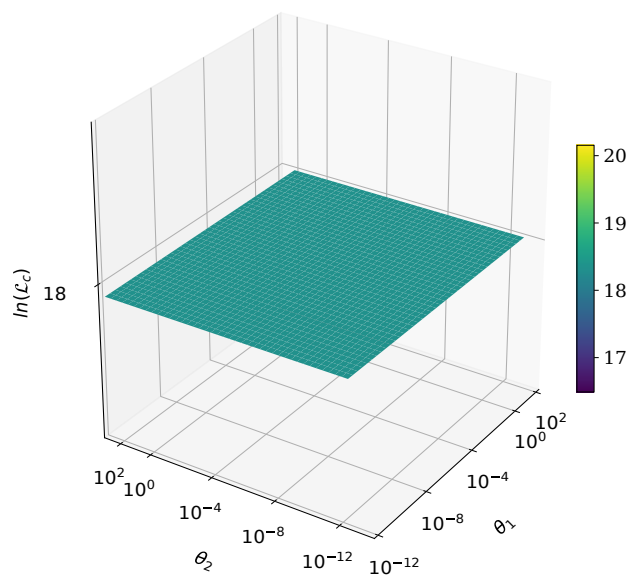

a) Linear MLE function

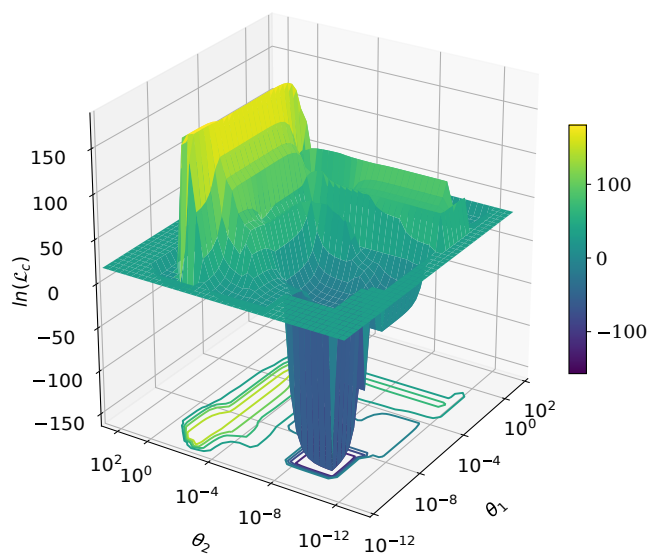

c) Logarithmic MLE function

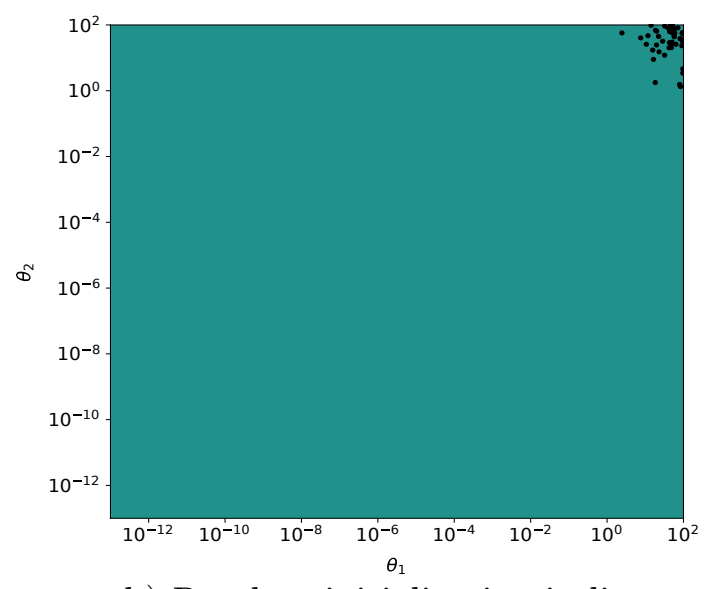

b) Random initialization in linear space (i.e. $\left.\theta_{\mathrm{h}}=1,2, \ldots, \mathrm{n}\right)$

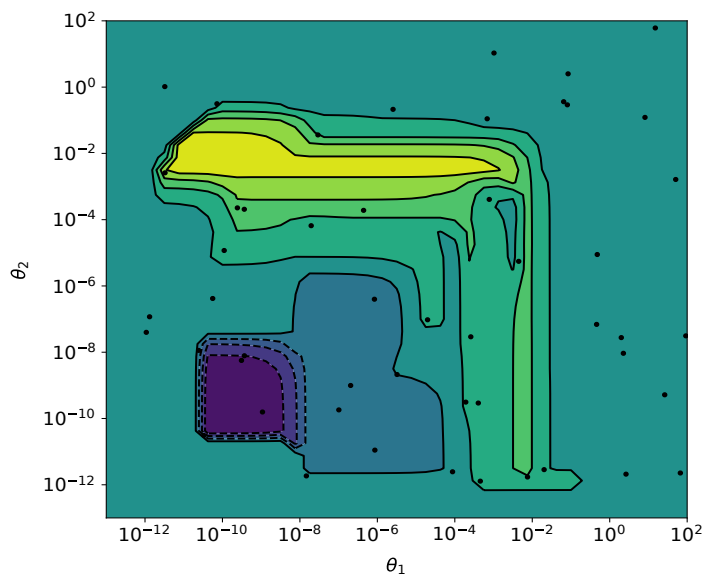

d) Random initialization in logarithmic space (i.e. $\theta_{\mathrm{h}}=10^{1}, 10^{2}, \ldots, 10^{\mathrm{n}}$ )

Figure 8.8: Effect of random uniform (top) and log-uniform (bottom) hyperparameter space initialization on the concentrated ln-likelihood function of Rosenbrock's $2 \mathrm{D}$ valley test function

Comparing the above figures, it is clear that a logarithmic coordinate system is better able to capture the complex topology of the ln-likelihood function while containing at least one local minimizer. The linear coordinate system appears to sample within a small region thereby resulting in large $\sim 0$ gradient portions within the functional space (as visible in Figure 8.8a). As such, a logarithmic coordinate system is selected to initialize the $\boldsymbol{\theta}$ hyperparameter weights in the current DACE model implementation. 


\section{Hyperparameter bounding}

In order to deal with the monotonicity associated with the MLE function, an upper and lower bound is typically placed on the model hyperparameter values. This is necessitated as the algorithm requires a finite range within which to operate. For the case of zero-approaching hyperparameter values, the lower limit is usually set very small as control is generally enforced by conditioning of the correlation matrix (later discussed in Section 8.5.3). Considering the converse case of infinitely increasing hyperparameter values, the upper limit is set sufficiently large while focus is rather given to ensuring a well distributed and adequate number of sample points. This has the effect of changing the behaviour of the MLE function such that local minimizers exist (Kok, 2012).

Following Bergh (2018), the bounds used in this investigation are chosen to be:

$$
\begin{aligned}
& b_{1}=10^{-12} \\
& b_{2}=10^{2}
\end{aligned}
$$

such that:

$$
b_{1} \leq \boldsymbol{\theta} \leq b_{2}
$$

Although these constraints technically place a limit on the maximum permissible value of the likelihood function, they are deemed satisfactory as none of the cases trialed in this investigation ever reached these limits. The only constraint found to affect the DACE model hyperparameter values was the constraint on the conditioning of the correlation matrix $\kappa(\widetilde{\mathbf{R}})$ (as introduced later in Equation 8.22).

\subsubsection{Regularization}

As previously mentioned, one of the major disadvantages of Kriging based methods is the likely instability associated with the computation of the inverse of the correlation matrix $\mathbf{R}^{-1}$. This is due to a near singularity in the matrix being caused by three possible sources, namely: 1) sample size, 2) input vector dimension, and 3) $\boldsymbol{\theta}$ parameter value (Peng and $\mathrm{Wu}, 2014)$. This can lead to model instability and consequently poor model performance as the inversion computation becomes more difficult with each subsequent prediction.

In order to measure the severity of this instability, the standard definition of the condition number for a matrix is introduced:

$$
\kappa(\mathbf{A})=\frac{\left|\lambda_{\max }(\mathbf{A})\right|}{\left|\lambda_{\min }(\mathbf{A})\right|},
$$


where $\lambda_{\max }$ and $\lambda_{\min }$ represent the maximum and minimum eigenvalues of the matrix A respectively. As a nonsingular matrix and its inverse have the same condition number, this metric can be used to determine the likely ill-conditioning of the latter if found to be very large. To put this into perspective when considering model accuracy, a good rule of thumb is to expect to lose at least $m$ digits of precision when computing the inverse matrix if $\kappa(\mathbf{A})=10^{m}$ (Cheney and Kincaid, 2012 ).

In order to better understand the effect of ill-conditioning of the correlation matrix $\mathbf{R}$, the substituted form of Equations 8.2 and 8.3 is given for convenience,

$$
\mathbf{R}=\exp \left[-\sum_{h=1}^{k} \theta_{h}\left|x_{h}^{i}-x_{h}^{j}\right|^{2}\right] .
$$

The effect of the $\boldsymbol{\theta}$ parameter on the correlation matrix is only considered as values for the sample size and input vector dimension are predefined and depend on the optimization problem. For ever increasing $\boldsymbol{\theta}$ parameter values $(\boldsymbol{\theta} \rightarrow \infty)$, the exponent of Equation 8.18 will increasingly become negative for all values outside the main diagonal (all diagonal entries will remain zero). Taking the exponential of this matrix, the correlation matrix will ever tend towards an identity matrix $(\mathbf{R} \rightarrow \mathbf{I})$ of which the condition number is $\kappa(\mathbf{A}) \sim 1$. This indicates a wellconditioned matrix whose approximate inverse solution is no less as accurate as that of the data.

Considering the converse with ever decreasing $\boldsymbol{\theta}$ parameter values $(\boldsymbol{\theta} \rightarrow 0)$, all entries in the exponent of Equation 8.18 will likewise tend to zero. This results in all entries of the correlation matrix tending towards one, with the condition number tending towards infinity $(\kappa(\mathbf{A}) \rightarrow \infty)$. This indicates an ill-conditioned correlation matrix which is consequently sensitive to perturbations. As such, this may result in the solution being dangerously in error.

\section{Nugget approach}

A common approach to ameliorate the detrimental effects caused by an ill-conditioned correlation matrix (as discussed above) is to add a small positive value $(v)$ along the diagonal of the matrix. In spatial statistics, $v$ is referred to as the nugget effect (Peng and $\mathrm{Wu}, 2014)$. The resulting regularized correlation matrix is defined as:

$$
\widetilde{\mathbf{R}}=\mathbf{R}+v \mathbf{I}_{n}
$$


where $\mathbf{I}_{n}$ is the identity matrix of size $n$. The eigenvalues of the regularized correlation matrix $(\widetilde{\lambda})$ are related to the eigenvalues of the standard correlation matrix ( $\lambda$ ) through $\tilde{\lambda}=\lambda+v$. When considering matrix stability, substituting $\tilde{\lambda}$ into Equation 8.17 and comparing with the standard form reveals:

$$
\left(\frac{\left|\lambda_{\max }(\mathbf{R})\right|+v}{\left|\lambda_{\min }(\mathbf{R})\right|+v}\right) \underset{\text { always }}{\leq}\left(\frac{\left|\lambda_{\max }(\mathbf{R})\right|}{\left|\lambda_{\min }(\mathbf{R})\right|}\right)
$$

for a given correlation length. This implies that $\widetilde{\mathbf{R}}$ is always better conditioned than $\mathbf{R}$. Additionally, for the situation when $\boldsymbol{\theta} \rightarrow \infty$, it can be shown that $\kappa(\widetilde{\mathbf{R}})=$ $\frac{n+v}{v}$ which guarantees that $\widetilde{\mathbf{R}}$ is always invertible provided that $n<\infty \sqrt{\text { Andri- }}$ anakis and Challenor, 2012).

As it has been shown that the value of the nugget can significantly influence the shape and modes of the likelihood function (Pepelyshev, 2010, Andrianakis and Challenor, 2012), careful consideration is taken in this investigation for its selection. Within the literature, there are two predominate means by which the value of the nugget is chosen, namely: restricted and unrestricted conditioning. The former, being the more conservative approach of the two, makes use of a very small nugget value (typically of the order $10^{-12}$ ) to reduce the forced error associated with introduction of the nugget. Consequently, due to such a small nugget value being used, this method requires an upper bound on the condition number of the correlation matrix to ensure stability. The disadvantage of this method is that this limit inadvertently places an upper bound on the value of the likelihood function.

The latter method requires no such limit on the condition number but instead increases the value of the nugget (typically of the order of $10^{-6}$ ) to ensure matrix stability. As previously explained, this approach has the inherent disadvantage of potentially increasing the forced error due to such a large nugget value. Table 8.4 compares the effect of the fitting characteristics of the DACE model obtained using the two aforementioned nugget values on the initial TE end-plate CFD database.

Table 8.4: Comparison of DACE model fitting characteristics with varying nugget value using the initial TE end-plate CFD database and DE algorithm solver

\begin{tabular}{ccccc}
\hline $\begin{array}{c}\text { Nugget value } \boldsymbol{v} \\
\text { (Source) }\end{array}$ & Max $\ln \left(\mathcal{L}_{\boldsymbol{c}}\right)$ & Max error & RMSE & $\boldsymbol{R}_{\text {std }}$ \\
\hline $4,142\left(10^{-5}\right)$ & -121.22 & 2.9834 & 0.0174 & 2.0365 \\
$($ Peng and $\mathrm{Wu}, 2014)$ & -121.22 & 2.9837 & 0.0174 & 2.0362 \\
\hline $9,324\left(10^{-15}\right)$ & & & & \\
\hline Lophaven et al. 2002$)$ & $202)$ & & & \\
\hline
\end{tabular}


As evident, although the larger nugget value of Peng and $\mathrm{Wu}(2014)$ provided for a lower maximum error, all other values are comparably similar and any differences are insignificant. As such, and likewise with that of former studies conducted using the DACE model (Bergh, 2018), the more conservative nugget value of Lophaven et al. (2002) is chosen for this investigation and set according to:

$$
v=(10+n) \epsilon_{m}
$$

where:

$\epsilon_{m}\left(=2^{-52} \approx 2,22 \times 10^{-16}\right)$ represents the floating-point relative accuracy of the machine.

For the current DACE model optimization routine, all computations were performed using a binary64 format (double precision) machine. Following Flannery et al. (1992), for a double precision system, a matrix can be considered ill-conditioned if its condition number exceeds $10^{12}$. Consequently, this value is chosen as the upper bound on the condition number for the regularized correlation matrix to ensure matrix stability. This constraint is represented by:

$$
\kappa(\widetilde{\mathbf{R}}) \leq 1 \times 10^{12}
$$

and is enforced by using the brick wall penalty method (as discussed in Section 2.7.1).

\subsection{The EGO algorithm}

As previously mentioned, the Efficient Global Optimization (EGO) algorithm of Jones et al. (1998) is used in conjunction with the current DACE model to complete the optimization routine. As suggested by its name, the EGO algorithm is intended for the optimization of expensive black-box type functions at a reduced number of function evaluations. The algorithm achieves this through the use of the socalled infill sampling criterion (the process of updating the model based on the introduction of additional sample points). An overview of the algorithm with specific reference to the current study is illustrated in Figure 8.9 . 


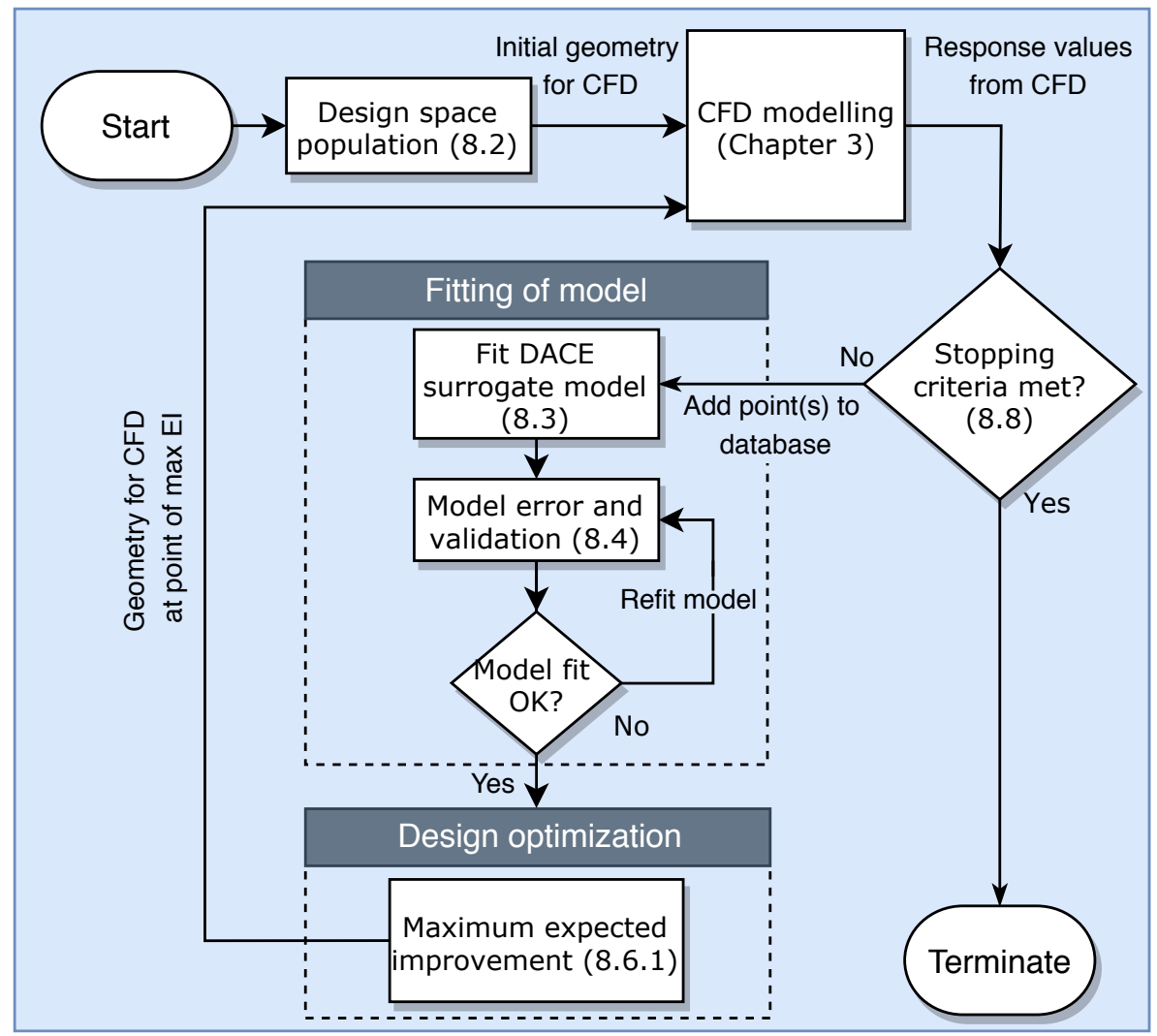

Figure 8.9: The EGO algorithm

The general steps of the algorithm are described below:

1. Fit the DACE model to an initial database of $n$ sampled points using maximum likelihood estimation.

2. Perform diagnostic tests on the model fit. If found unsatisfactory, re-fit the DACE model by applying a log or inverse $(-1 / y)$ transformation to the response values. If unable to find satisfactory fit, find alternative model to fit to the data.

3. Once satisfactory fit is obtained, determine the point at which the infill sampling criteria is optimal.

4. Evaluate the true value of the objective function at the optimal infill sample point and update the database with $n+1$ points.

5. Repeat steps 1 through 4 until specified stopping criteria is met. 


\subsubsection{Expected improvement}

Various infill sampling criteria have been developed to ensure appropriate selection of additional sample points. Of these, the simplest method would be to evaluate the minimum of the response surface and iterate. However, as shown in Figure 8.10, iterating through this method can easily lead to a highly accurate yet sub-optimal solution in the presence of local minima.

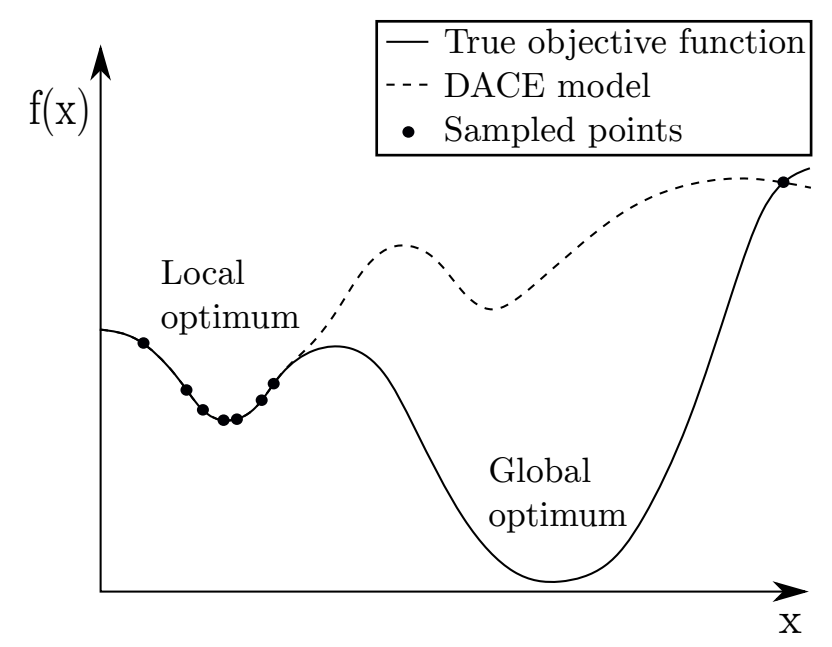

Figure 8.10: Simple sampling of the response surface

As indicated by Jones et al. (1998), this is a result of the process not acknowledging the uncertainty associated with the response surface as a result of poor model generalization when sampling the design space discretely. In order to subvert these problems, the EGO algorithm makes use of the generalized expected improvement (EI) function. With this function, the algorithm attempts to balance between a local and global search criteria whereby preference is given to points which have a low predicted objective function value and (importantly) high model uncertainty which correlates to a large model inaccuracy.

The improvement of the current best point is defined by:

$$
I=\max \left(0, f_{\min }^{n}-Y\right)
$$

where:

$f_{\min }^{n}$ is the lowest objective function value within the current database, and

$Y$ measures the uncertainty at $y(\mathbf{x})$ by treating it as a realization of a normally distributed random variable with $N\left(\hat{y}, s^{2}\right)$. 
Expressing the right hand side of Equation 8.23 as an integral and performing integration by parts, the expected improvement can be expressed in closed form as

$$
E(I)= \begin{cases}\left(f_{\min }^{n}-\hat{y}\right) \Phi \frac{f_{\min }^{n}-\hat{y}}{\hat{s}}+\hat{s} \phi \frac{f_{\min }^{n}-\hat{y}}{\hat{s}} & \text { if } s \geq 0 \\ 0 & \text { if } s=0 .\end{cases}
$$

where:

$\hat{y}(\mathbf{x})$ and $\hat{s}(\mathbf{x})$ are the predicted and $R M S E$ estimates from Section 8.4 . respectively, and

$\Phi$ and $\phi$ represent the standard normal cumulative distribution function (cdf) and probability density function (pdf), respectively.

Inspection of Equation 8.24 reveals the first term to represent the likelihood of improving the current best minimum multiplied by the difference between the current and predicted minimum values. The second term represents the probability of the current predicted value being equal to the current best minimum value multiplied by the standard deviation of $y(\mathbf{x})$. This all equates to the EI function returning high values when $\hat{y}$ is predicted to be less than that of the current best minimum and/or where there is a high uncertainty associated with the current predicted value. Importantly, this also means that the EI function will return a value of zero at known sampled locations.

\section{WB1 criteria}

Although this balance between local and global search is what makes the EI function highly attractive, it is for this same reason that this function provides inadequacies during the later stages of optimization. For instance, once the algorithm has confidently detected the region in which the optimum is located, the EI function should (ideally) focus all of its attention within this region to best maximize the improvement on the current best point. Instead, the EI function will move away from this region in an attempt to further explore regions of only moderate interest thereby delaying algorithm convergence.

One alternative criteria that resolves the previously mentioned deficiencies is that of the WB1 criteria for locating threshold-bounded extremes as proposed by Watson and Barnes (1995). This function presents a more local search criteria in that it attempts to maximize the probability of exceeding a specified threshold. The WB1 function in the context of design optimization is defined by Sasena et al. (2002) as: 


$$
\mathrm{WB} 1=\Phi\left(\frac{f_{\min }^{n}-\hat{y}}{\hat{s}}\right)
$$

where:

$\Phi, f_{m i n}^{n}, \hat{y}$ and $\hat{s}$ are defined as previously.

\section{Generalized expected improvement}

Following Sasena et al. (2002), a generalized form of the EI function is introduced by including a non-negative integer parameter, $g$, into Equation 8.23 to give

$$
I^{g}=\max \left(0,\left(f_{\min }^{n}-Y\right)^{g}\right),
$$

Repeating the integration procedure as performed previously, the generalized form of the expected improvement function is given as

$$
E\left(I^{g}\right)= \begin{cases}s^{g} \sum_{k=0}^{g}(-1)^{k}\left(\frac{g !}{k !(g-k) !}\right)\left(f_{\min }^{\prime n}\right)^{g-k} T_{k} & \text { if } s \geq 0 \\ 0 & \text { if } s=0 .\end{cases}
$$

where:

$$
\begin{aligned}
& f_{m i n}^{\prime n}=\frac{f_{m i n}^{n}-\hat{y}}{\hat{s}}, \text { and } \\
& T_{k}=-\phi\left(f_{\text {min }}^{\prime n}\right)\left(f_{m i n}^{\prime n}\right)^{k-1}+(k-1) T_{k-2}
\end{aligned}
$$

starting with $T_{0}=\Phi\left(f_{m i n}^{\prime n}\right)$ and $T_{1}=-\phi\left(f_{m i n}^{\prime n}\right)$.

As explained by Sasena et al. (2002), increasing the $g$ parameter has the effect of shifting the emphasis towards a more global search criteria while lower values represent a more local search criteria. Figures 8.11 and b illustrate the significance of the $g$ parameter on the EI function by comparing the originally introduced EI function (represented by setting $g=1$ ) and the WB1 criteria of Watson and Barnes (1995) (represented by setting $g=0$ ). 


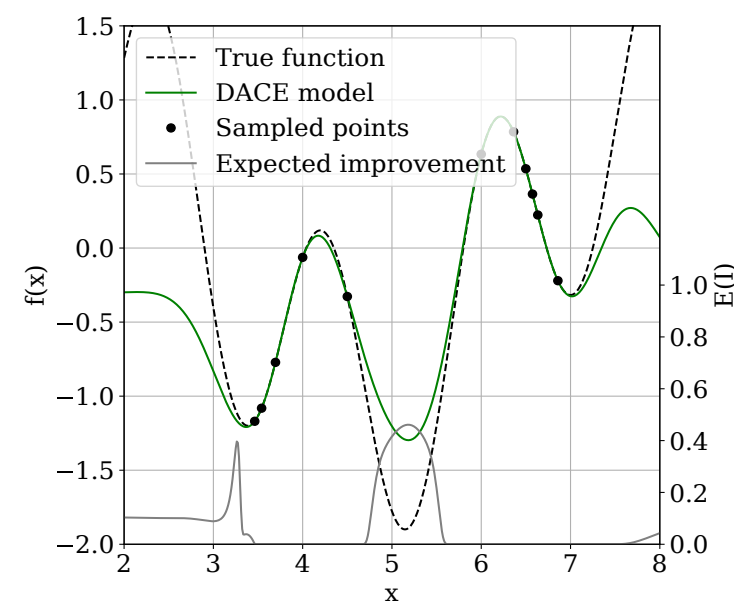

a) Expected improvement $(\mathrm{g}=1)$

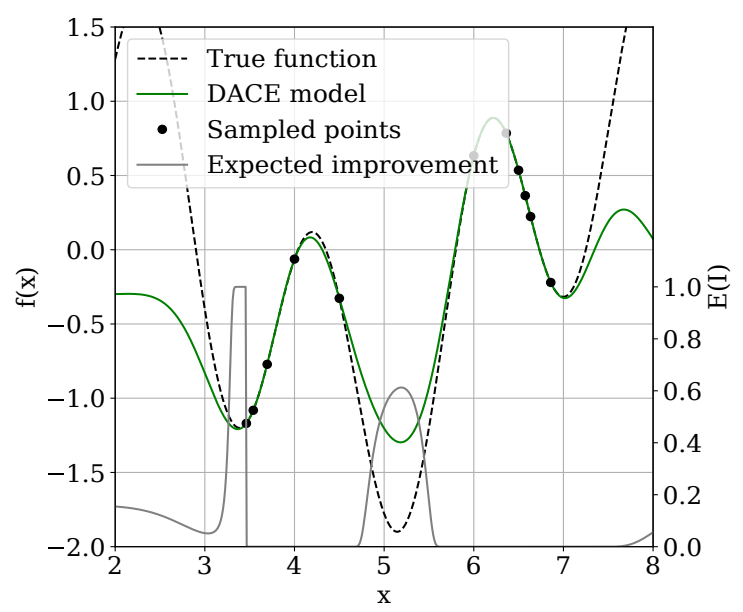

b) Expected improvement $(\mathrm{g}=0)$

Figure 8.11: Influence of $g$ parameter on EI function for a simple 1-D function with $f(x)=\sin (x)+\sin (10 x / 3)$

In order to take advantage of the aforementioned strategies, a modified version of the cool criterion of Sasena et al. (2002) is used in this investigation to systematically locate the optimum. In this method, the original EI criteria $(g=1)$ is first used to locate the region within which the optimum lies. Once confident that the optimal region has been located, the WB1 criteria $(g=0)$ is used to locally refine the search space by sampling threshold-bounded extremes within this region.

The stopping criteria of this method is based on the number of EGO iterations rather than a set convergence criteria. This is purely due to a computational time restriction and is elaborated on in Section 8.8. The final EGO cooling schedule used in the current investigation is given in Table 8.5 .

Table 8.5: EGO cooling schedule

\begin{tabular}{cc}
\hline Iteration & g value \\
\hline $1-17$ & 1 \\
$18-34$ & 0 \\
\hline
\end{tabular}

\subsubsection{Maximum expected improvement estimation}

In their original work, Jones et al. (1998) propose the use of a branch-and-bound algorithm for maximization of the EI criterion. In this method, determination of a lower bound on $\hat{y}$ and an upper bound on $\hat{s}$ - denoted $y^{L}$ and $s^{U}$ respectively - 
is required before computation of the EI function through substitution into Equation 8.24. However, although this method is able to guarantee optimality of the multimodal EI function, difficulties associated with computation of the two additional sub-problems along with computation of the EI function make this method impractical (Bergh, 2018). Additionally, it is unclear how this method is applied to constrained optimization problems.

A more practical approach to maximization of the EI criteron would be to make use of the DE algorithm of Price et al. (2005) already implemented in this investigation (see Section 2.5.2). Although this method may not guarantee optimality of the EI function, it is a well known global optimization algorithm which can easily be applied to constrained optimization problems. Observing these deficiencies, the DE algorithm is set to perform a minimum of 1000 iterations of the EI function before checking for convergence. Likewise with Bergh (2018), this is done in order to prevent premature convergence of the algorithm to a local optimum (especially along the flat portions of the EI objective space).

\subsection{Algorithm validation}

In order to employ the aforementioned DACE model and EGO algorithm combination, algorithm validation is required to compare the performance of the optimization routine. This is done in order to asses suitability with regards to its use in the current study. The following sections outline the validation process.

\subsubsection{Definition of performance metrics}

Before validation can be performed, a set of reference performance metrics need to be defined in order to be able to adequately measure algorithm performance. Following Sasena et al. (2002) and Bergh (2018), the performance metrics used to validate the current optimization routine are introduced as follows:

$f\left(\hat{x}^{*}\right)$ - The function value at the known global optimal solution.

$f\left(x^{*}\right)$ - The function value at the best obtainable solution.

$f_{n}\left(x^{*}\right)$ - The ratio between the number of function evaluations at which the best obtainable solution was found and the total number of allowable function evaluations.

$x_{1 \%}$ - The number of function evaluations before a point is sampled within a region that spans $\pm 1 \%$ of the design space range (on a component-wise basis) centralized around the known optimal point. 
$f_{1 \%}$ - The number of function evaluations before a point returns a value that lies within $1 \%$ of the true minimum objective function value.

$\left\|\hat{\mathbf{x}}^{*}-\mathbf{x}^{*}\right\|$ - The Euclidean distance from the known global optimal point $\left(\hat{\mathbf{x}}^{*}\right)$ to the best obtainable solution $\left(\mathrm{x}^{*}\right)$.

\subsubsection{Test functions}

The following table presents four unconstrained optimization test problems used to validate the current EGO algorithm.

Table 8.6: Summary of test functions used in EGO algorithm validation

\begin{tabular}{|c|c|c|c|}
\hline$\overline{\text { Type }}$ & Test function & $\begin{array}{l}\text { DACE } \\
\text { transformation }\end{array}$ & Attributes \\
\hline \multicolumn{4}{|l|}{ Unimodel } \\
\hline & Sum Squares Function & None & Bowl-shaped function \\
\hline & Rosenbrock Function & Logarithmic & Narrow, parabolic valley function \\
\hline \multicolumn{4}{|l|}{ Multimodal } \\
\hline & Michalewicz Function & None & $\begin{array}{l}\text { Deceptive function with steep } \\
\text { ridges/drops }\end{array}$ \\
\hline & Ackley 1 Function & None & $\begin{array}{l}\text { Hugely multimodel function with } \\
\text { many local optima }\end{array}$ \\
\hline
\end{tabular}

Each function was evaluated on their respective standard hypercubes, as suggested by Jamil and Yang (2013). These functions present well known benchmark test functions used to validate the performance of different optimization algorithms. Their selection was based on their ability to imitate different scenarios which could potentially relate to the current optimization problem. These scenarios include:

- A trivial case which provides for relatively easy convergence to the optimal solution (Sum Squares Function).

- The presence of narrow, flat regions of the objective space as a result of minor changes to the shape of the TE end-plate design. Although easy to locate the valley, Rosenbrock's test function provides for difficulty when converging to the minimum.

- A deceptive function which offers little information to the location of the global optimum as a result of radical changes to the response surface (Michalewicz function, $m=10$ ). Such an instance could relate to a severe end-plate setting angle for the TE end-plate design (as identified in Section 6.3.2). 
- Ackley's 1 function which presents a severely multimodal function with many local minima. This function presents a particularly difficult case to solve as the optimization algorithm may become stuck in one of the many local minima, thereby increasing convergence time or cause the algorithm to not be able to reach convergence at all.

\subsubsection{Results}

In each case, with Michalewicz's function being the exception, both the two dimensional and three dimensional (same dimension as the current study's optimization problem) alternatives were tested to determine the effect of varying the problem dimensionality on the solution. A maximum of 20 EGO iterations for each test function is chosen for evaluation as this provided for sufficient results when using the same algorithm on a set of similar test functions (Bergh, 2018).

Additionally, as discussed in Section 8.6.1, two criterion for the computation of expected improvement are trialed for each case. These include:

1. The standard EI criterion as suggested by Jones et al. (1998), and

2. A modified version of the cool approach of Sasena et al. (2002) which makes use of an evenly weighted contribution from both the standard EI criterion (10 iterations) and WB1 criterion (10 iterations).

Table 8.7 compares the various performance metrics of the EGO algorithm using the aforementioned criteria against the previously mentioned test problems. The final three columns represent the metrics of the criteria that were best able to predict the functions global optimal value $f\left(\hat{\mathbf{x}}^{*}\right)$.

Table 8.7: Comparison of optimizer performance metrics against four different test functions

\begin{tabular}{|c|c|c|c|c|c|c|c|c|c|}
\hline \multirow[b]{2}{*}{ Type } & \multirow[b]{2}{*}{ Test function } & \multirow[b]{2}{*}{$\mathbf{f}\left(\hat{\mathbf{x}}^{*}\right)$} & \multicolumn{2}{|c|}{ Standard EI } & \multicolumn{2}{|c|}{$\mathrm{EI}+\mathrm{WB1}$} & \multirow[b]{2}{*}{$\mathrm{x}_{1} \%$} & \multirow[b]{2}{*}{$f_{1 \%}$} & \multirow[b]{2}{*}{$\| \hat{\mathbf{x}}^{*}-\mathrm{x}^{*}$} \\
\hline & & & $f\left(x^{*}\right)$ & $\mathbf{f}_{\mathbf{n}}\left(\mathrm{x}^{*}\right)$ & $f\left(x^{*}\right)$ & $\mathbf{f}_{\mathbf{n}}\left(\mathrm{x}^{*}\right)$ & & & \\
\hline \multicolumn{10}{|l|}{ Unimodel } \\
\hline & Sum Squares (2D) & 0 & 0.0000 & $1 / 20$ & N.A. & N.A. & 1 & 1 & 0.0289 \\
\hline & Sum Squares (3D) & 0 & 0.0754 & $1 / 20$ & N.A. & N.A. & 1 & 1 & 0.2258 \\
\hline & Rosenbrock (2D) & 0 & 0.7753 & $19 / 20$ & 0.0464 & $13 / 20$ & 8 & 1 & 0.0176 \\
\hline & Rosenbrock (3D) & 0 & 45.7447 & $16 / 20$ & 3.1211 & $20 / 20$ & N.A. & 20 & 1.8969 \\
\hline \multicolumn{10}{|l|}{ Multimodal } \\
\hline & Michalewicz (2D) & -1.8013 & -1.7911 & $18 / 20$ & -1.8009 & $20 / 20$ & 12 & 12 & 0.0078 \\
\hline & Ackley 1 (2D) & 0 & 0.3972 & $20 / 20$ & 0.1148 & $20 / 20$ & 9 & N.A. & 0.0612 \\
\hline & Ackley 1 (3D) & 0 & 1.7602 & $19 / 20$ & 1.1786 & $16 / 20$ & 1 & N.A. & 1.2567 \\
\hline
\end{tabular}


As evident, the trivial case of the Sum Squares function did not present the algorithm with a very challenging problem. The algorithm was able to obtain a very accurate solution on the first iteration for both problem dimensions. Comparing the results between the two cases, a key conclusion that can be drawn is the apparent decrease in solution accuracy as a result of increased problem dimensionality. The same effect can be seen in the other test problems.

Comparing Rosenbrock's test function, although both EI criteria were able to obtain relatively accurate solutions for the two dimensional case, only the EI + WB1 criterion was able to obtain an acceptable solution for the higher dimension case. The algorithm was found to struggle when sampling the flat portions of the objective space, particularly with such severe changes of the response surface near the optimum. This demonstrates the importance of including some form of local search criteria when locating optima.

Considering Michalewicz function, both criteria were able to obtain highly accurate solutions, again with the EI + WB1 criterion offering superior performance. Although the algorithm obtained its best solution near the iteration limit for both cases, the algorithm was found to sample within $1 \%$ of the region surrounding the global optimum within 12 iterations.

Finally, considering Ackley's function, the EI + WB1 criterion once again performed best; with a final predicted function value of $\sim 0,1$ located at a Euclidean distance of 0.0612 away from the true global optimum for the two dimensional case. Considering the three dimensional case, the algorithm experienced difficulty in locating the true optimum. Interesting to note when comparing the two cases, despite the higher dimension case obtaining a relatively less accurate solution it was able to sample within $1 \%$ of the optimal region on the first iteration. This demonstrates the effect of the size of the initial database as the higher dimension case contains more sampled locations thereby reducing the required number of iterations to sample near the optimum.

Despite not being able to achieve all performance metrics, the optimization routine was found to perform satisfactory for all test functions with relatively small errors. These findings concur with that found by Bergh $(2018)$ who completed a similar validation procedure. Based on the above results, the current optimization routine is deemed sufficient for use in the current study. 


\subsection{Stopping criteria}

As suggested by Jones et al. (1998), if the EI function produces a result of less than $1 \%$ of the best current response value (untransformed scale), convergence is deemed sufficient and the EGO algorithm is consequently stopped. However, due to large CFD simulation times encountered during the datum fan analysis, following this procedure for the current optimization problem would be too time intensive. This is due to the requirement of a large number of EGO algorithm iterations in order to meet the aforementioned convergence criteria thereby making optimization impractical. As a result, the convergence criteria used in the current investigation is based off an iteration/time constraint as opposed to a specified change in response value.

As noted in the previous section, increased problem dimensionality has the effect of increasing the required initial database size which consequently increases the overall optimization time. In order to gain insight into an acceptable range for the proposed iteration limit, the relevant literature on the DACE model is considered for evaluation. Table 8.8 summaries the relevant information of recent studies that made use of the DACE model in design optimization.

Table 8.8: Summary of relevant literature on DACE model stopping criteria

\begin{tabular}{|c|c|c|}
\hline$\overline{\text { Author }}$ & Problem dimensionality & $\overline{\text { Iterations }}$ \\
\hline 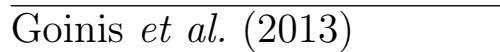 & $11 \mathrm{D}$ & 300 \\
\hline Jeong et al. $(2006)$ & $11 \mathrm{D}$ & 43 \\
\hline Bergh $(2018)$ & $12 \mathrm{D}$ & 300 \\
\hline Jeong and Obayashi $(2005)$ & $26 \mathrm{D}$ & 63 \\
\hline Deng et al. (2013) & $33 \mathrm{D}$ & 350 \\
\hline
\end{tabular}

The total number of EGO iterations used in the current study is based on the maximum number that still allows for optimization to be completed within a realistic time frame. Considering the relevant literature in Table 8.8, the temporal requirements of the various stopping criteria are normalized with respect to the current study's problem dimensionality and given in Table 8.9 (with the average being indicated in red). 
Table 8.9: Temporal requirements for the TE end-plate optimization using relevant DACE model literature with $k=3$

\begin{tabular}{l|ccc}
\hline Author & Iterations & Final database size & $\begin{array}{c}\text { Total predicted } \\
\text { simulation time [days] }\end{array}$ \\
\hline Jeong and Obayashi & 2005 & & 16.1 \\
\hline Jeong et al. (2006) & 12 & 43 & 23.7 \\
\hline Deng et al. & $2013)$ & 47 & 65.6 \\
\hline Average & 32 & 67 & 70.1 \\
\hline Bergh (2018 & 34 & 110 & 159.7 \\
\hline Goinis et al. & 75 & 117 & 175.2 \\
\hline
\end{tabular}

The average provides for an intermediate number of optimization iterations when compared to the literature and equates to a value similar to the initial end-plate database size (or more specifically around $11 k$ additional points). This would mean a total optimization time of just over 2 months for the current optimization problem which is considered conservative given its relatively low dimensionality. As such, a total of 34 optimization iterations are chosen for optimization of the TE end-plate design. 


\section{Chapter 9}

\section{End-plate optimization results}

This chapter presents the numerical results of the end-plate optimization process. Validation of the initially populated database is first carried out in order to asses suitability for optimization. Thereafter, design convergence is analysed in order to evaluate the performance of the optimization routine and determine sampling adequacy. Finally, the Pareto-front of the objective function space is presented in order to select the best possible set of competing solutions.

\subsection{Optimizer cross-validation}

As previously mentioned, validation of the DACE model with respect to the current optimization problem is required in order to ensure sufficient minimization of the models error and uncertainty. As such, the relevant cross-validated information of the LOO analysis from Section 8.4 is presented in Figure 9.1 for the current DACE model, along with a summary of the achieved errors. 


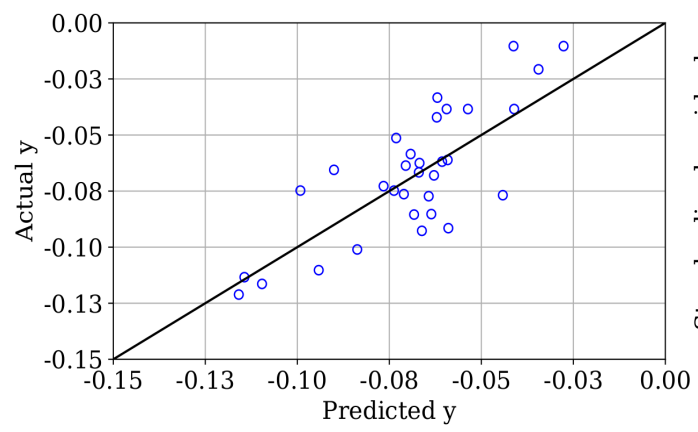

a) $y_{\text {actual }}$ vs $y_{\text {predicted }}$

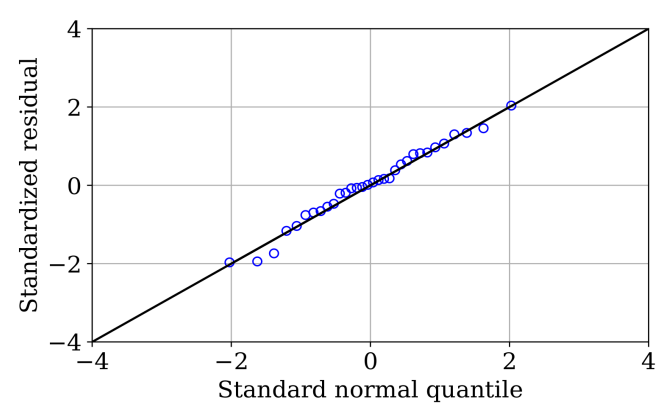

c) Q-Q plot

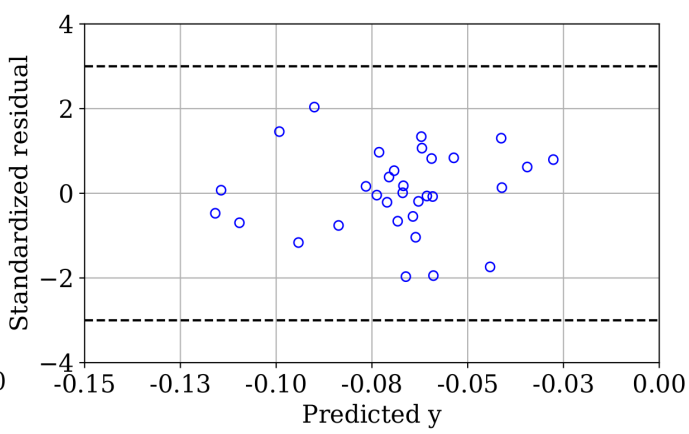

b) Standardized residuals $\left(R_{\text {std }}\right)$

\begin{tabular}{cccc}
\hline $\mathbf{n}_{\text {points }}$ & Max error & RMSE & Max $\mathbf{R}_{\text {std }}$ \\
\hline 32 & 2.9837 & 0.0174 & 2.0362 \\
\hline
\end{tabular}

d) Error summary

Figure 9.1: DACE model validation of the TE end-plate design optimization

As evident, the current database illustrates an even distribution of actual vs predicted response values around the $45^{\circ}$ dividing line. When considering the relevant errors, although the maximum error is significant, a closer inspection reveals that this is due to the presence of low objective function values. This would cause a magnification of the error which would lead to a skewed interpretation of the results. The latter is supported by a sufficiently low $R M S E$ value and Q-Q plot distribution. These findings are aligned with that found in the relevant literature (Bergh, 2018).

The database's maximum standardized residual is well within the recommended range (as suggested by Jones et al. (1998)) to ensure sufficient validation. As a result of the above, the current DACE model database with 32 data points is deemed satisfactory for use in the current optimization study.

\subsection{Convergence history}

As discussed in Section 8.8, the convergence criteria of the current optimization routine is based on a maximum iteration/time constraint which makes use of a 
modified version of the cool criterion of Sasena et al. (2002). In this method, the sampling effort is uniformly subdivided between two criteria which systematically refine the search from a more global search criteria to that of a local one. Figure 9.2 illustrates the convergence of the objective function $F(x)$ of the current optimization routine using the aforementioned criteria (denoted OPTI 1 and OPTI 2 respectively).

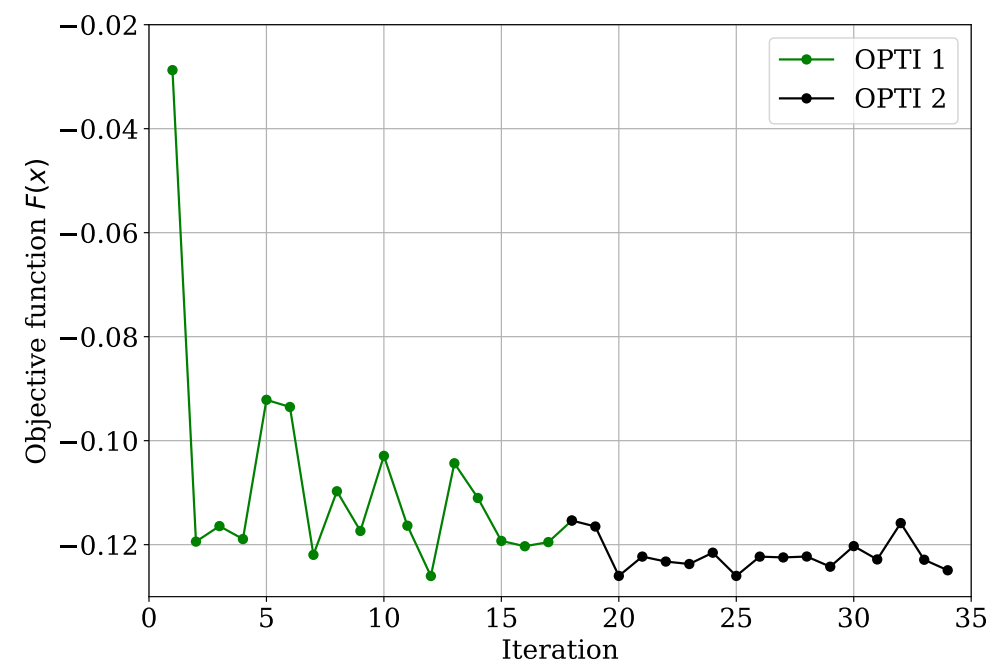

Figure 9.2: Convergence history of the TE end-plate optimization routine

As illustrated, the response values of OPTI 1 exhibit large fluctuations as a result of the EI criteria being more bias towards searching areas of greater uncertainty. Nonetheless, a steady decrease in response values is observed throughout this phase; during which the magnitude of the fluctuations are also found to decrease. Thereafter, the WB1 criteria is selected to perform the second optimization phase in OPTI 2. As predicted, the second phase is found to show a steadier trend in response values due to the more local search criteria giving greater attention to threshold-bounded extremes. Overall, a consistent reduction in response values is observed throughout the end-plate's optimization with the three best response values differing by only $0.015 \%$.

Figure 9.3 gives a spatial scatter plot representation of the end-plate's optimization in order to gain insight into the overall convergence as well as the presence of any local optima that may be of interest for alternative designs. Additionally, the trajectory of the optimization routine is given in three-dimensional space to illustrate the algorithm's development. 


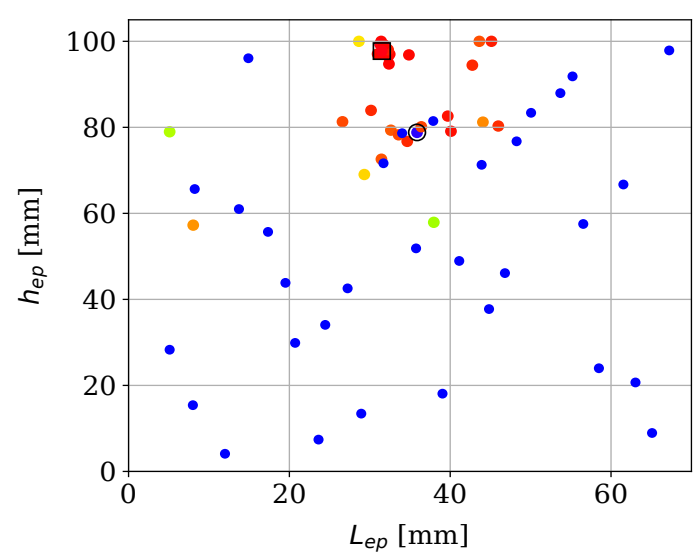

a) $L_{e p}-h_{e p}$ projection plane

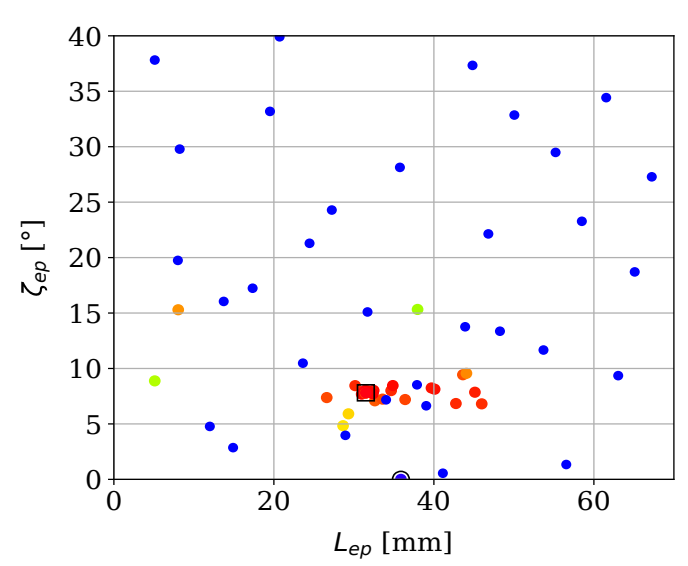

c) $L_{e p}-\xi_{e p}$ projection plane

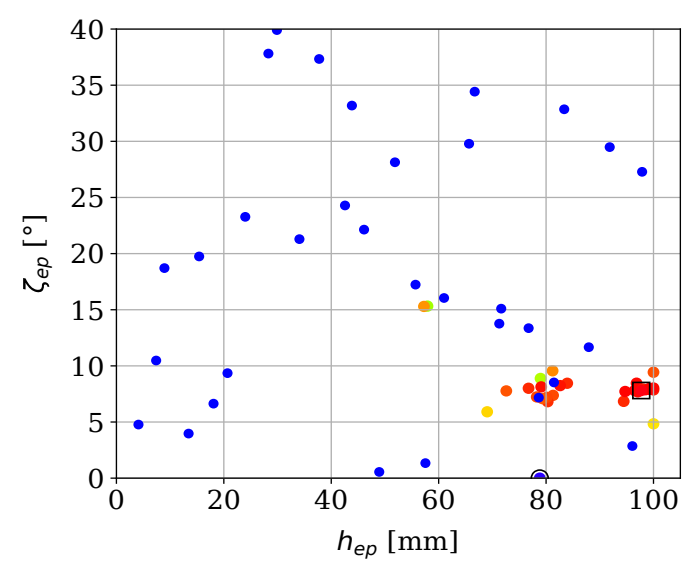

b) $h_{e p}-\xi_{e p}$ projection plane

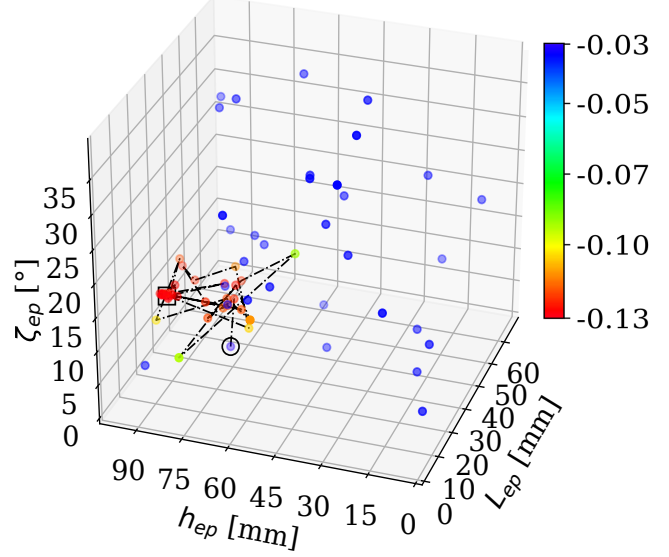

d) Optimized $\mathbf{X}_{\text {database }}$

Figure 9.3: Spatial convergence history of TE end-plate optimization: Initial database (blue dots), start point (o), end point $(\square)$

As evident in the algorithm's trajectory (Figure 9.3 d), the optimization routine was able to quickly refine the search to a relatively localized region within the design space; only deviating a few times to explore exterior regions. This further illustrates the adequacy of the initial database as the requirement for explorative points was reduced.

Interesting to note in Figure $9.3 \mathrm{~b}$ is the clustering of points around the $80 \mathrm{~mm}$ and $95 \mathrm{~mm}$ locations for the $h_{e p}$ parameter with both having a $\zeta_{e p}$ angle of around $7.5^{\circ}$. This supports the notion that the two controlling variables are most influential on the response value as both have well defined local regions of optimality. 
Although the algorithm was found to sample along the $h_{e p}$ parameter boundary, these sampled points were few as the majority of the sampled points were located within the design space bounds. This illustrates the adequacy of the design space bounds estimation performed in Section 7.2 .2 .

\subsection{Pareto-front}

Figure 9.4 shows the Pareto-front of the multi-objective, multi-point end-plate optimization process. The horizontal axis gives the total-to-static efficiency improvement over the datum fan while the vertical axis gives the slope between the efficiencies of the two flow rates (a lower slope indicating a higher efficiency at lower flow rates).

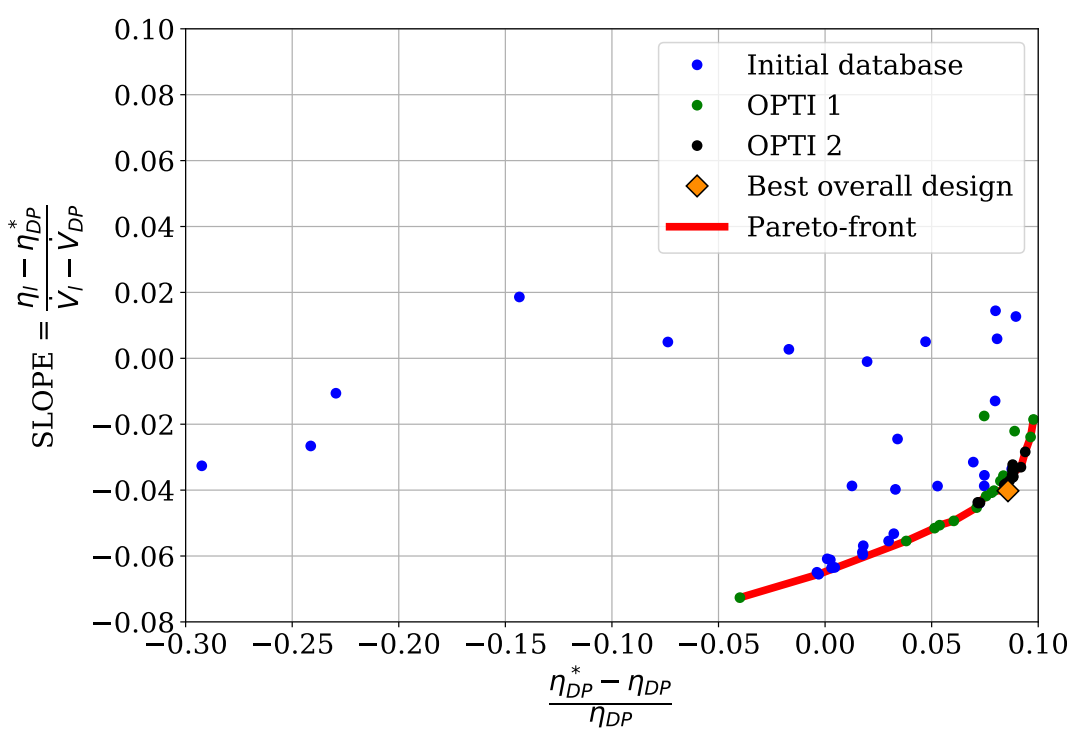

Figure 9.4: Pareto-front of multi-objective, multi-point TE end-plate optimization process

As shown, the Pareto-front of non-dominated solutions is clearly visible with the majority of the points being contributed by OPTI 2. A local clustering of points is evident around the point of maximum curvature along the front, which is synonymous with the location of the optimal design. This indicates sufficient sampling of the optimal region as no other solutions were found to better compromise between competing objectives. Additionally, the location of the best overall design's $\zeta_{e p}$ parameter value is aligned with the location of the minimum moment coefficient 
found in the relevant flat plate literature (Figure 6.9). This further reinforces confidence in the location of the optimal design. Consequently, this point is chosen for further analysis.

\subsection{Final selected designs}

Additional to the selection of the overall best design, a modified version of the design (with a zero $\zeta_{e p}$ parameter value) is selected for further analysis. This stems from the TE end-plate aerodynamic performance analysis performed in Section 6.3.2. The findings indicated that larger end-plate setting angles present reduced performance characteristics at lower than DP flow rates. Consequently, this design is chosen with the aim of investigating increased performance at lower flow rates while at the same time showing the effect of only increasing the blade's tip chord length. Details of the relevant designs are illustrated in Figure 9.5 and Table 9.1 below.

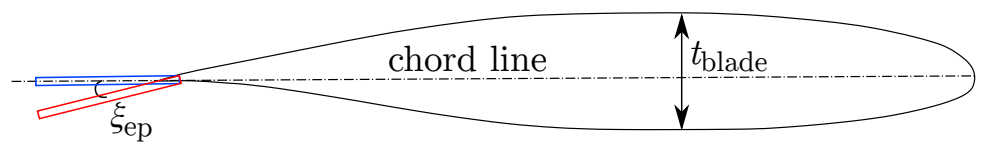

Blade tip

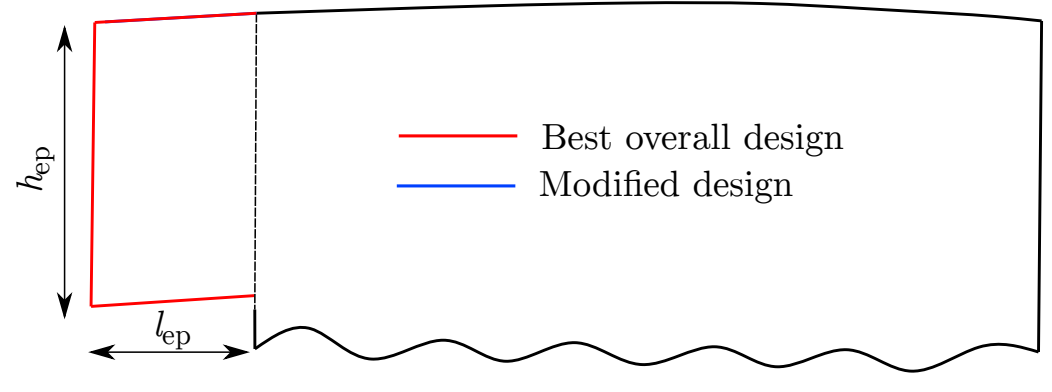

Figure 9.5: Final selected designs

Table 9.1: Final selected designs

\begin{tabular}{cccc}
\hline Design & $\mathbf{l}_{\mathbf{e p}}[\mathrm{mm}]$ & $\mathbf{h}_{\mathbf{e p}}[\mathrm{mm}]$ & $\boldsymbol{\zeta}_{\mathbf{e p}}\left[^{\circ}\right]$ \\
\hline Best overall & 31.58 & 97.06 & 7.81 \\
Modified & 31.58 & 97.06 & 0.00 \\
\hline
\end{tabular}




\subsection{Chapter summary}

The following points summarize the numerical results of the TE end-plate optimization process.

- Firstly, a validation study involving the initial end-plate database is performed to determine suitability for optimization when using the current optimization algorithm. The findings indicate acceptable model fit when considering the RMSE and $\mathrm{Q}-\mathrm{Q}$ plot distribution of the data. Although a high maximum error was observed, a closer inspection revealed that this was due to the presence of low objective function values thereby resulting in a magnification of the errors. Considering the above, the initial database is deemed satisfactory for use in the current optimization process.

- Various convergence metrics are presented in order to gain insight into the algorithm's convergence history as well as determine sampling adequacy. A consistent reduction in response values is observed throughout the first optimization phase (OPTI 1). Thereafter, a steadier trend in response values is observed in the second optimization phase (OPTI 2) which is synonymous with a local clustering of points around the optimum. This was further supplemented by two-dimensional spatial scatter plot representations which show an additional clustering of points around the $80 \mathrm{~mm}$ location for the $h_{e p}$ parameter. Although some points were sampled near the $h_{e p}$ parameter bounds, these were few as the majority of points were sampled well within the design space bounds. The final three best response values were found to differ by only $0.015 \%$.

- Finally, the Pareto-front of non-dominated solutions is presented. The findings clearly indicate the location of the optimal point which coincides with the point of maximum front curvature as well as the previously identified point clustering. Considering the relevant flat plate literature, the location of the minimum moment coefficient of a similar sized flat plate is found to concur with the location of the optimal design. This further reinforces confidence in the location of the optimum. Consequently, this point is chosen for further analysis. Additionally, a modified version of this design is selected with the aim of improving the fan's low flow rate performance characteristics. 


\section{Chapter 10}

\section{Experimental method}

Experimental analyses of the various end-plate designs are required in order to verify the numerical results and obtain the full characteristic performance curves. The following sections detail the design's construction, the fan test facility, fan testing procedures, and finally the numerical and experimental aerodynamic performance results of the chosen end-plate designs.

\subsection{Design construction}

As it is desired to minimize the weight of the blade as far as practical, the working material for the design should be selected with this in mind. For the same reason, the design itself should be attached in such a way as to introduce as little new material as possible while still adhering to the design requirements set out in Section 6.1

Considering this, the material used in the design's construction will be chosen to be the same as that used in the blade's construction i.e. carbon fibre. More specifically, a biaxial $\left(+/-45^{\circ}\right)$ variant is selected as this material offers a high strength-to-weight ratio with a resistance against torsional and twisting stresses making it ideal for the current application. The method used in the design's construction is presented in Appendix E, with an illustration of the resulting design given in Figure 10.1 


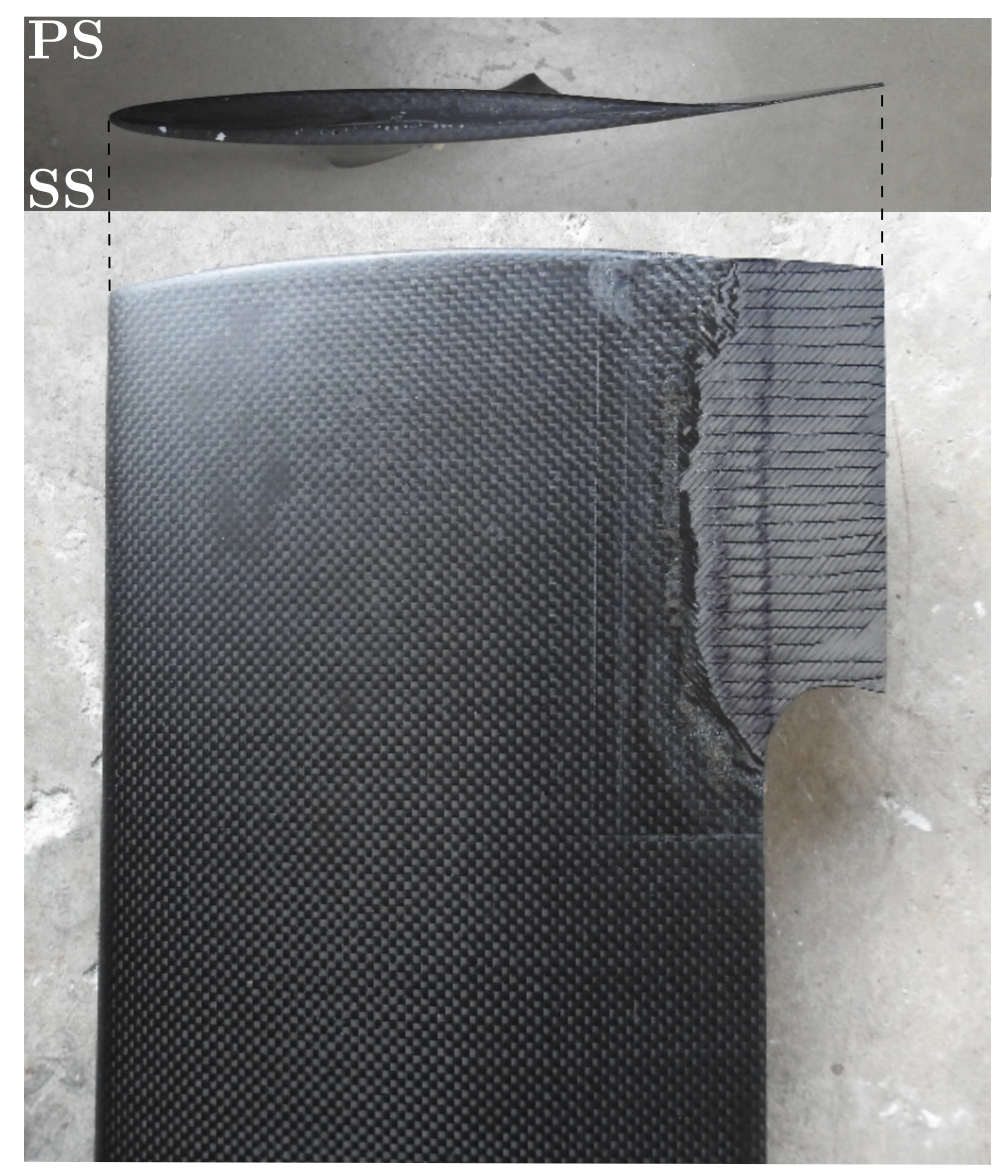

Figure 10.1: Final end-plate design

Additionally, a slow hardening epoxy resin (SR 1280 / SD 4775) is used to reinforce the carbon fibre elements and bond the laminated composite. Using the numerical results from Chapter 9, the structural feasibility analysis of the optimized TE end-plate design is presented in Appendix $\mathrm{F}$

\subsection{Fan performance testing}

\subsubsection{Test facility}

The test facility used in the current investigation is that of the ISO 5801, type A (open inlet-outlet) test facility at Stellenbosch University. This facility is used to gauge the performance of various types of axial flow fans by measuring their total-to-static pressure rise $\left(\Delta p_{t-s}\right)$ and power consumption $(P)$ which can then be used to calculate the fan's total-to-static efficiency $\left(\eta_{t-s}\right)$. These measurements 
are taken over a wide range of volumetric flow rates $(\dot{V})$ to describe the fan's stable operating envelope. An illustration of the test facility is given in Figure 10.2 .

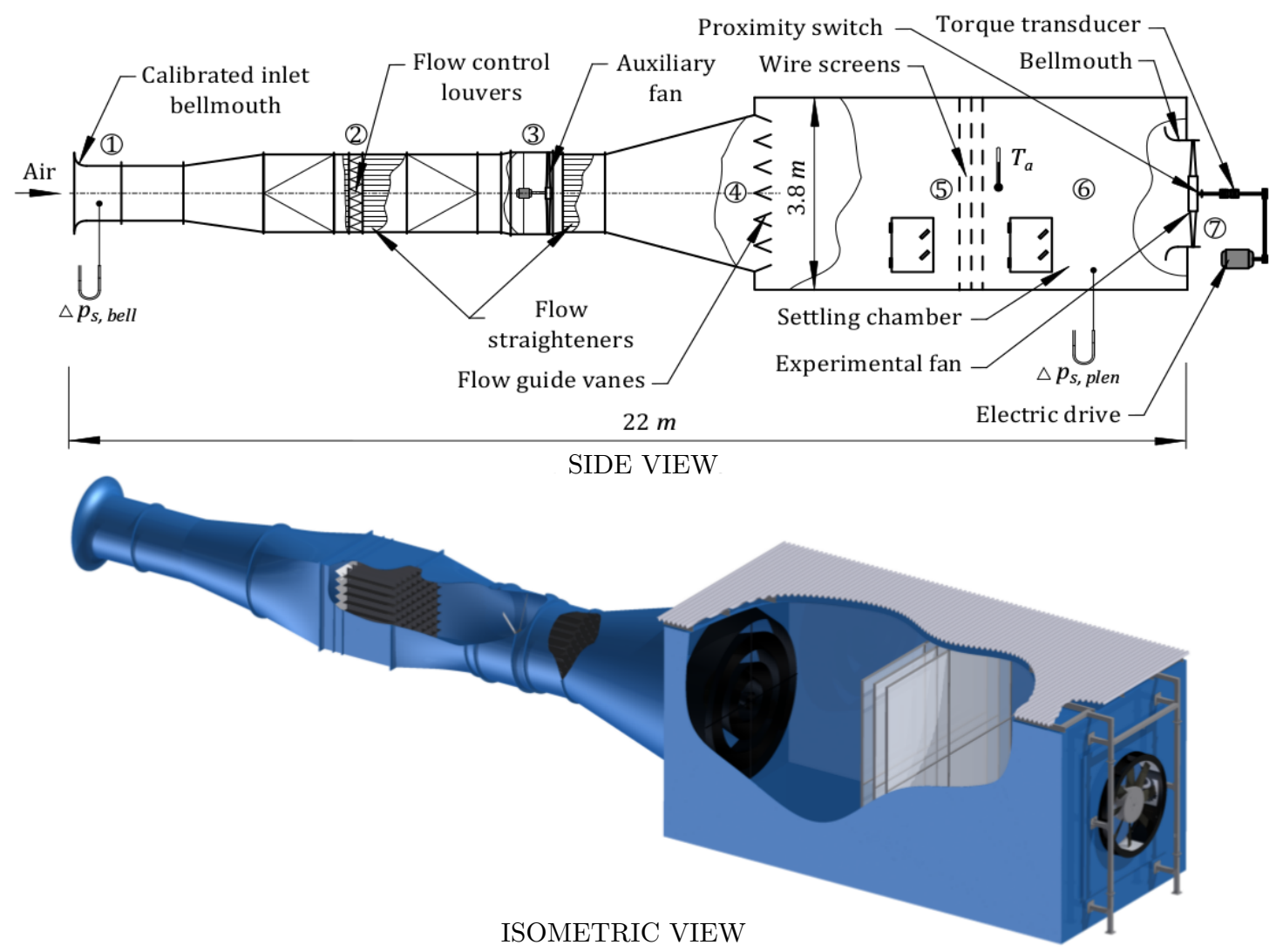

Figure 10.2: Stellenbosch University ISO 5801, type A test facility (adapted Louw (2015))

Once the facility is in operation, air is drawn in through the inlet bellmouth at location (1). A set of louvers are used to control the amount of airflow at (2), after which the air is straightened via the use of flow straighteners. An auxiliary fan is included at (3) to aid the airflow in overcoming the mechanical losses experienced at the higher volumetric flow rates. Thereafter, the flow is again straightened via a set of flow straighteners before being expanded into the settling chamber. A set of flow guide vanes located at the entrance of the settling chamber (4) ensure the incoming flow has a uniform distribution. This uniform distribution is further supplemented by the three wire screens located at (5), after which the air is allowed to enter the final part of the settling chamber at (6). 
The test fan, located at (7), is formed by connecting each blade to a central jig which uses indexing plates to aid in the blade setting angle adjustment to be at the required angle of $\zeta_{h}=34^{\circ}$ (see Wilkinson et al. (2019)). The tip clearance is set to $\tau=6 \mathrm{~mm}$ by adjusting the blade diameter through the use of five $1 \mathrm{~mm}$ thick spacers located between each indexing plate and jig block (default tip clearance being $1 \mathrm{~mm}$ ). Once the blades are set in place, Correx sheets are used to form the central hub. Silicone is additionally used to ensure a good seal between the blade root and central hub to prevent flow leakage. Finally, the full fan assembly is mounted onto the driveshaft which is located concentrically to the fan casing and fan inlet bellmouth. The driveshaft is driven by an electrical drive system which is connected to a variable speed drive. An example of the test fan set-up is shown in Figure 10.3 .

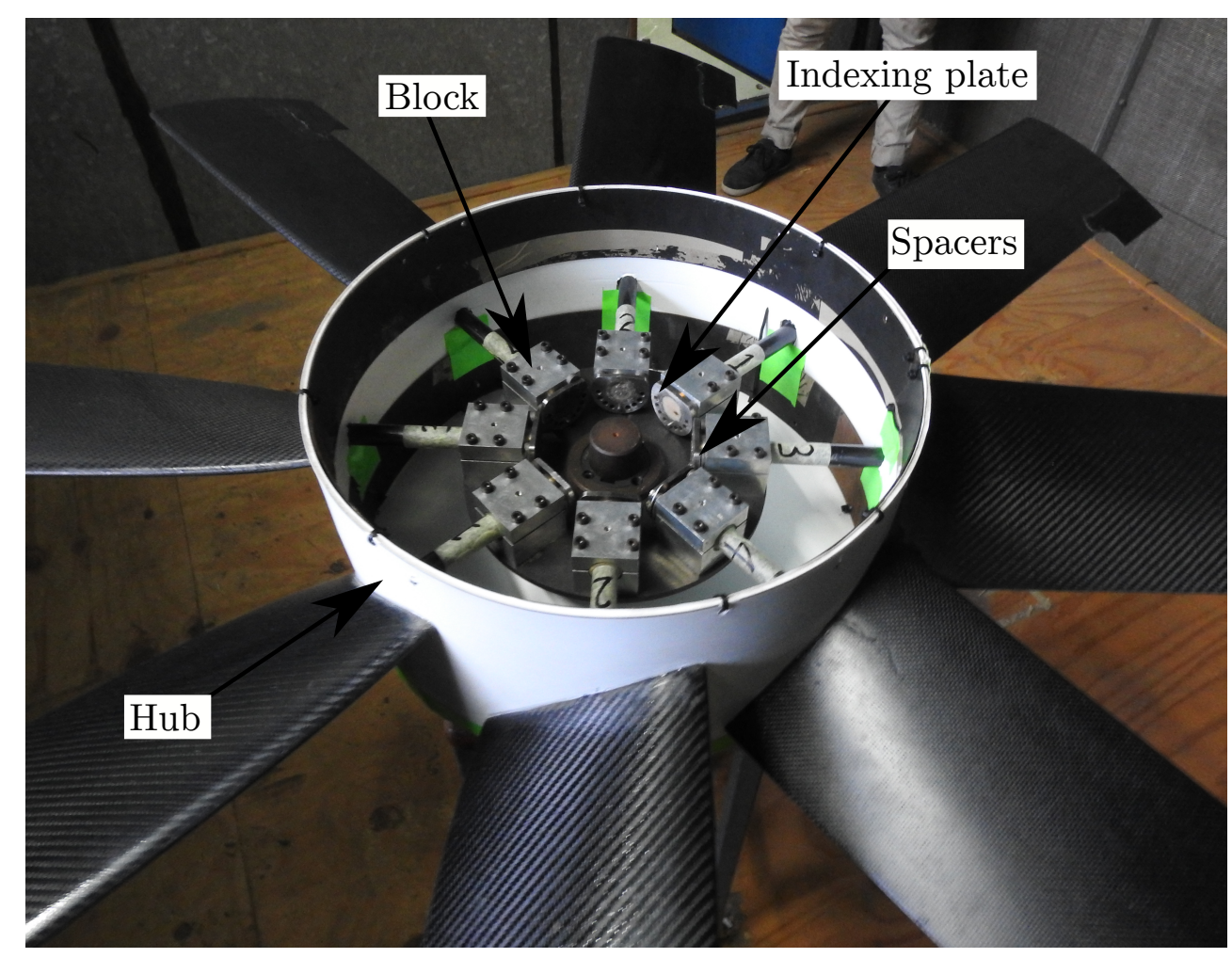

Figure 10.3: Test fan set-up

\subsubsection{Facility instrumentation}

During a single test run, the facility monitors four values at each flow rate, namely:

1. Static inlet bellmouth gauge pressure $\left(\Delta p_{s, b e l l}\right)$ 
2. Static plenum gauge pressure $\left(\Delta p_{s, p l e n}\right)$

3. Fan torque $(T)$

4. Fan rotational speed $(N)$

The inlet bellmouth and plenum chamber pressure taps, located at points (1) and (6) in Figure 10.2 respectively, are connected to two EH Deltabar PMD75 pressure transducers for the static pressure measurements. Fan torque is measured via a HBM T22 torque transducer which is located downstream of the fan and connected to the fans driveshaft. Finally, fan speed is measured through a proximity switch which measures the time delay between the gaps of a square toothed gear located on the driveshaft. The subsequent frequency output is then transformed through the use of a frequency-to-voltage converter. All measurement devices are fed into a central HBM PMX data acquisition system which is interfaced with a desktop computer through the use of the CATMAN software package. Details of the system are given in Figure 10.4 and Table 10.1, respectively.

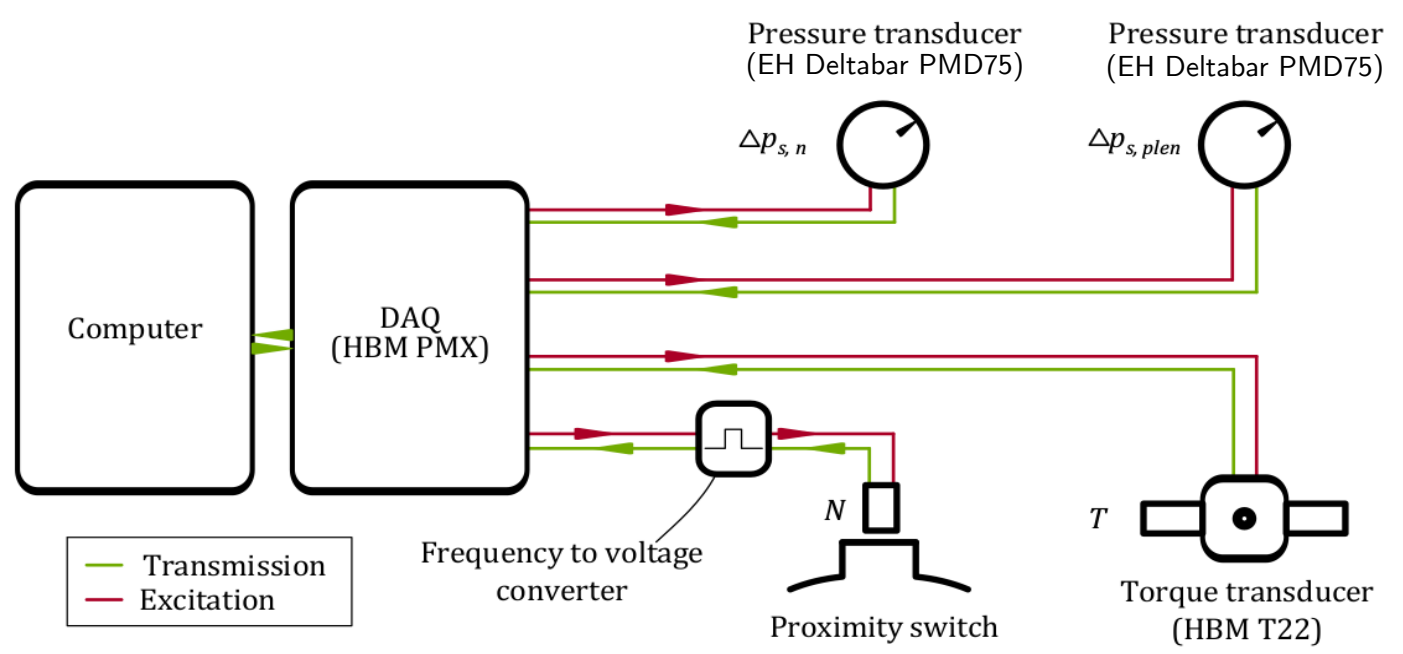

Figure 10.4: Test facility instrumentation acquisition system (adapted from Louw, 2015)

Table 10.1: Test facility instrumentation errors (Endress and Hauser Group, 2020. Louw, 2015)

\begin{tabular}{ccc}
\hline Instrument & Operating range & Nominal \\
\hline Pressure transducer (EH Deltabar PMD75) & $\pm 1000 \mathrm{~Pa}$ & $\pm 0,36 \%$ \\
Torque transducer (HBM T22) & $0-100 \mathrm{~N} . \mathrm{m}$ & $\pm 1,1 \%$ \\
\hline
\end{tabular}


Each of the four instruments are calibrated prior to testing. An example of such a calibration is given in Appendix G. Additional instrumentation include a dry bulb thermometer located inside the plenum chamber and a barometer located inside an adjacent building. These instruments, along with the data obtained during each test run, are used to calculated the fan characteristic performance metrics as demonstrated in Appendix $\mathrm{H}$.

\subsubsection{Testing procedure}

Prior to testing, and without the fan assembly being mounted, the test facility is brought up to design speed $\left(N_{m}=722 \mathrm{rpm}\right)$ and run for at least 10 min before taking a zero torque reading. This is done in order to capture the no load operating torque $\left(T_{N L, f}\right)$ of the test facility as this was found to vary considerably during start-up.

Once complete, the test facility is shut down before the fan assembly is securely mounted onto the driveshaft. The front hub is covered by more Correx sheet and the edges are sealed to ensure no leakage. Thereafter, the initial ambient air temperature and pressure $\left(T_{a, i}, P_{a, i}\right)$ are recorded separately and the remaining zero measurement readings are taken. The facility is brought up to design speed before setting the control louvres to the initial position and recording the time taken to capture the first operational measurement $\left(\mathrm{t}_{\text {start }}\right)$. Using the CATMAN software package, all instrument values are recorded over a 30 second interval at a rate of $100 \mathrm{~Hz}$ before being stored. This process is repeated while systematically going through each louvre position to obtain a new volumetric flow rate. Once a flow rate of around $45 \%$ of design point flow rate is achieved, the auxiliary fan is switched on to compensate for the mechanical losses experienced at all higher flow rate measurements. Data acquisition is stopped once the plenum chamber pressure $\left(p_{s, p l e n}\right)$ obtains a value close to zero. The facility is then switched off, after which the final zero reading is taken as before along with the final ambient air temperature and pressure readings $\left(T_{a, f}, P_{a, f}\right)$. Thereafter, the data is downloaded for post-processing and analysed to obtain the fan's performance characteristics.

Once the fan has undergone a sufficient number of tests to confirm repeatability (with three for each test case being deemed satisfactory for this investigation) the fan assembly is removed from the driveshaft. Using the previously recorded start time $\left(\mathrm{t}_{\text {start }}\right)$, the test facility is yet again brought up to design speed and the initial no load torque $\left(T_{N L, i}\right)$ measurement is recorded. As previously mentioned, this is due to the torque varying considerably during start-up and therefore needs to be taken into consideration. 


\subsection{Results}

\subsubsection{Aerodynamic performance analysis}

Figures 10.5 through 10.7 compare the experimental and numerical fan performance characteristics of the final selected TE end-plate designs with that of the datum fan for a $6 \mathrm{~mm}$ tip clearance. As described in Section 9.4, these include the optimal end-plate design (denoted "best overall design") and a modified version of the optimal design (denoted "modified design"). As done previously, the same operational points are chosen for the numerical comparisons while the experimental results give the full operational envelope.

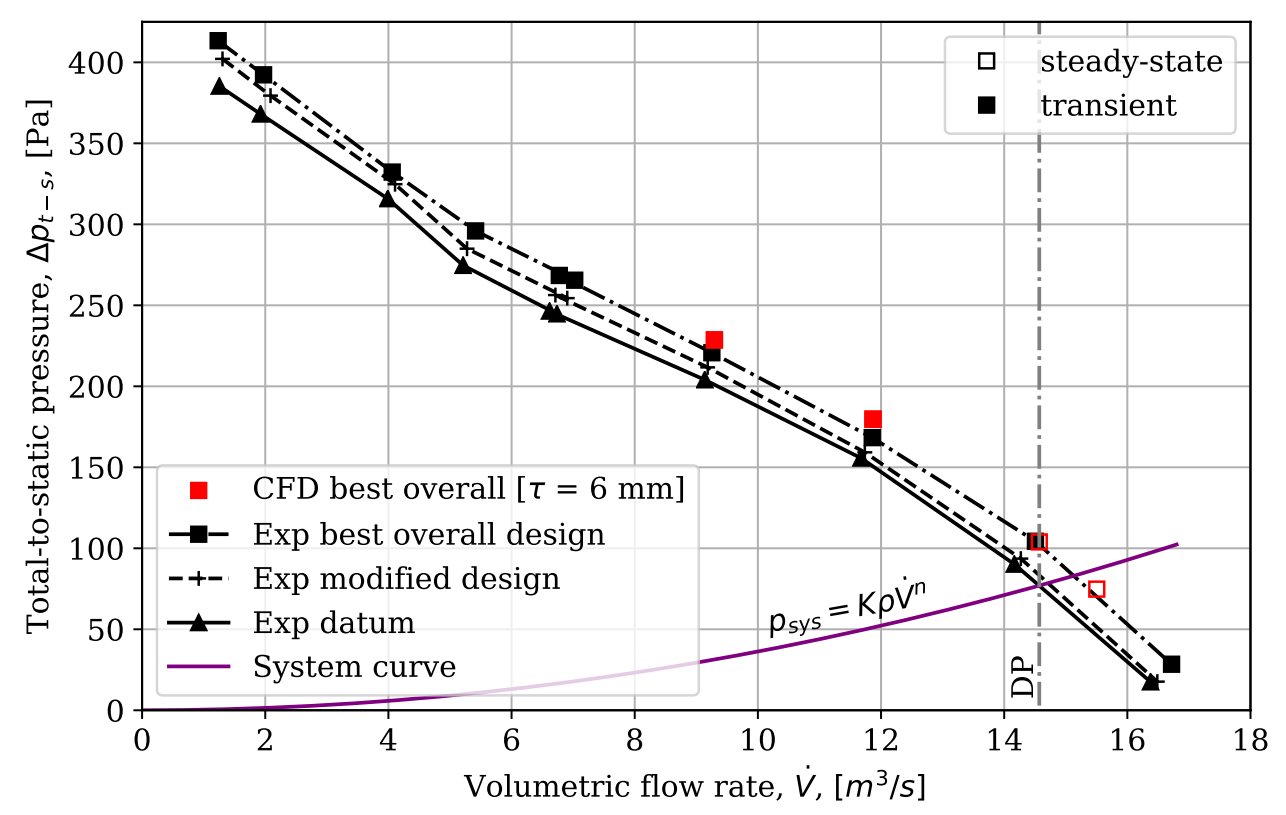

Figure 10.5: Experimental and numerical trailing edge end-plate and datum fan total-to-static pressure rise comparison 


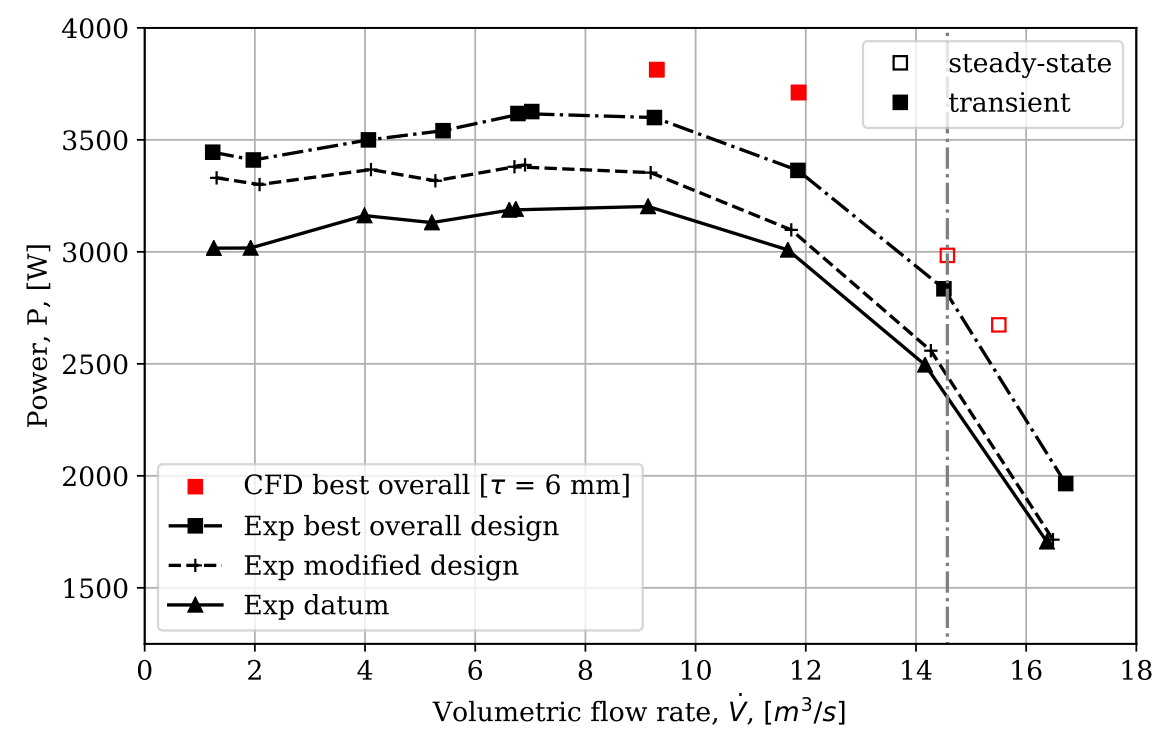

Figure 10.6: Experimental and numerical trailing edge end-plate and datum fan power comparison

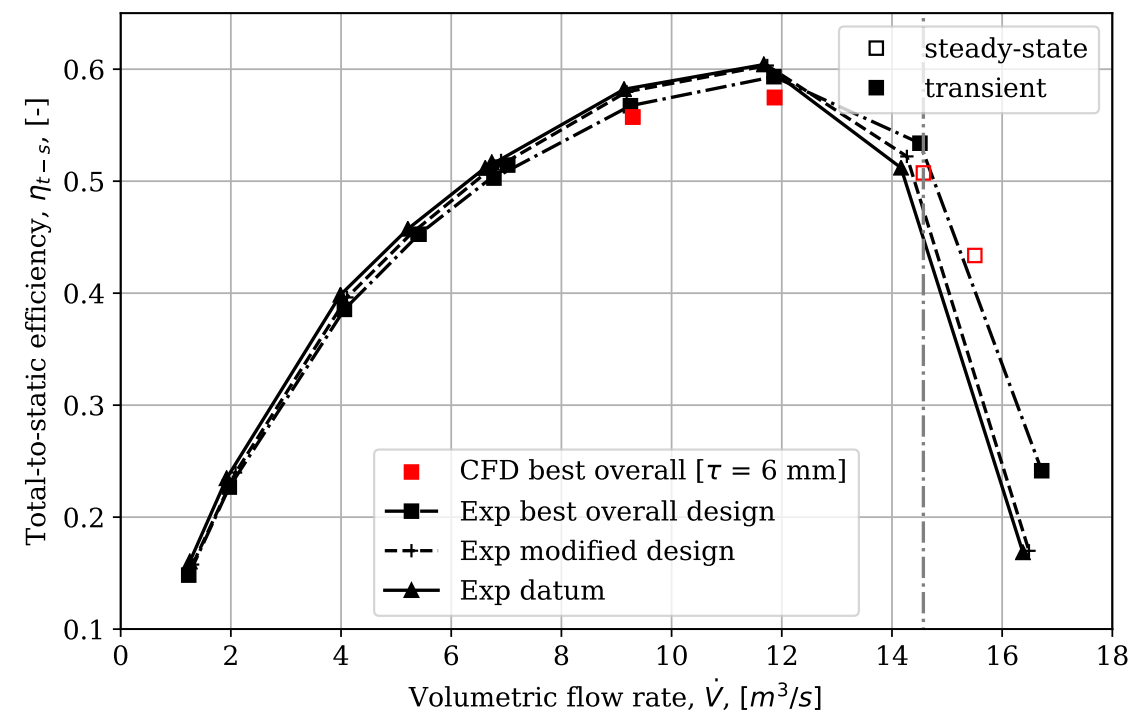

Figure 10.7: Experimental and numerical trailing edge end-plate and datum fan total-to-static efficiency comparison 
Comparing the numerical and experimental results of the best overall design, good correlation is found when considering the fan's total-to-static pressure rise characteristics. However, the same can not be said when considering the fan's power requirement. As previously observed in the datum fan analysis, the numerical solver struggles to accurately predict the fan's power leading to a gross overestimation. Despite these inadequacies, the fan's numerical total-to-static efficiency characteristics are found to give good correlation to the experimental results. The results are found to be well within the acceptable range when considering the validation study conducted in Chapter 4. This further illustrates the appropriateness of the proposed meshing strategy and numerical set-up when modelling the M-fan.

Comparing only the experimental fan performance curves, the best overall design is found to improve the datum fan's total-to-static pressure rise characteristics across the entire evaluated operating range with a 32.90 percent increase at the fan's design point flow rate. However, this increase in pressure is met with a subsequent increase in the fan's power requirement. Despite this, the optimized end-plate design is found to improve the fan's total-to-static efficiency at higher than peak efficiency flow rates with an indicated increase of 7.66 percentage points at the fan's design point flow rate.

When considering the effect of only increasing the blade chord ("modified design"), a similar net effect on the fan's performance characteristics is found when compared to that of the best overall design (albeit at reduced improvement margins). The modified design is found to achieve its design goal by improving the low flow rate efficiency characteristics when compared to the best overall design. However, this is met with a reduced improvement margin for greater than peak efficiency flow rates. As previously mentioned, this is due to a greater end-plate setting angle requirement for higher than peak efficiency flow rates.

These findings support the post-processing CFD analysis performed in Section 6.3. where the existence of a specific end-plate setting angle that provides for effective control of the TLV exit trajectory angle was proposed. This is indeed found to be the case when considering the current two end-plate designs which show a reduced end-plate setting angle requirement at lower volumetric flow rates. These findings suggest that the end-plate's setting angle and volumetric flow rate are directly related to improving the fan's performance.

\subsubsection{Operating point comparison}

As noted in the above section, the introduced end-plate designs are found to increase the fan's total-to-static pressure rise characteristics at the fan's design point 
flow rate. However, an increase in fan pressure has the inherent consequence of shifting the fan's operating point further towards the right as a result of increased pressure losses. An illustration of this can be seen by comparing the intersection points between the fan's performance curve and system curve in Figure 10.5. Consequently, this needs to be taken into consideration to be able to make accurate comparisons between designs. Table 10.2 quantitatively indicates the corrected design point performance characteristics of the various end-plate designs against the fan datum.

Table 10.2: Experimental fan performance characteristics of trailing edge end-plate designs compared to fan datum evaluated at each design's operational flow rate for a $6 \mathrm{~mm}$ tip clearance

\begin{tabular}{ccccc}
\hline & \multicolumn{4}{c}{ Variable } \\
Design & $\dot{\boldsymbol{V}}\left[\mathrm{m}^{\mathbf{3}} / \mathrm{s}\right]$ & $\boldsymbol{\Delta}_{\boldsymbol{t}-\boldsymbol{s}}[\mathrm{Pa}]$ & Power $[\mathrm{W}]$ & $\boldsymbol{\eta}_{\boldsymbol{t}-\boldsymbol{s}}[-]$ \\
\hline datum $[\tau=6 \mathrm{~mm}]$ & 14.57 & 76.94 & 2351.40 & 0.449 \\
Best overall & 15.13 & 82.99 & 2590.46 & 0.452 \\
Modified & 14.71 & 78.48 & 2390.41 & 0.452 \\
\hline
\end{tabular}

As apparent, both end-plate designs are still found to improve the fan's performance characteristics (albeit at reduced improvement margins). The optimized end-plate design is found to increase the datum fan's pressure rise by 7.86 percent and the total-to-static efficiency by 0.3 percentage points. This illustrates that the optimized end-plate design is able to improve the fan's pressure rise characteristics without sacrificing on efficiency.

Considering the effect of increasing the blade chord, it is interesting to note that the modified design is able to achieve a similar total-to-static efficiency to that of the optimized end-plate design. This is significant as this design is able to improve the fan's performance characteristics at higher than peak efficiency flow rates with an almost negligible performance loss at lower volumetric flow rates (unlike that of the optimized design). This is more than likely a result of the increased chord improving the vortex loss behaviour near the blade tip while simultaneously reducing the end-plate's power requirement at lower flow rates when compared to the optimal design. This further illustrates the capabilities of the TE end-plate design and shows that its characteristics should be tailored to specific operating conditions (i.e. better high flow rate performance at a reduced low flow rate performance or vice versa). 


\subsubsection{Angle of attack analysis}

As this design effectively increases the AoA at the blade tip, it would make sense to compare this design to the effect of simply increasing the blade's stagger angle. The optimized end-plate design (which makes use of a box hub configuration with a blade setting angle of $34^{\circ}$ ) is found to increase the relative AoA at the blade tip by $1.2^{\circ}$ when compared to the fan datum. Therefore, using linearly interpolated values from Wilkinson et al. (2019) to match on a case by case basis (that being for a $6 \mathrm{~mm}$ tip clearance with a box hub), the results comparison can be visualized in Figures 10.8 to 10.10 .

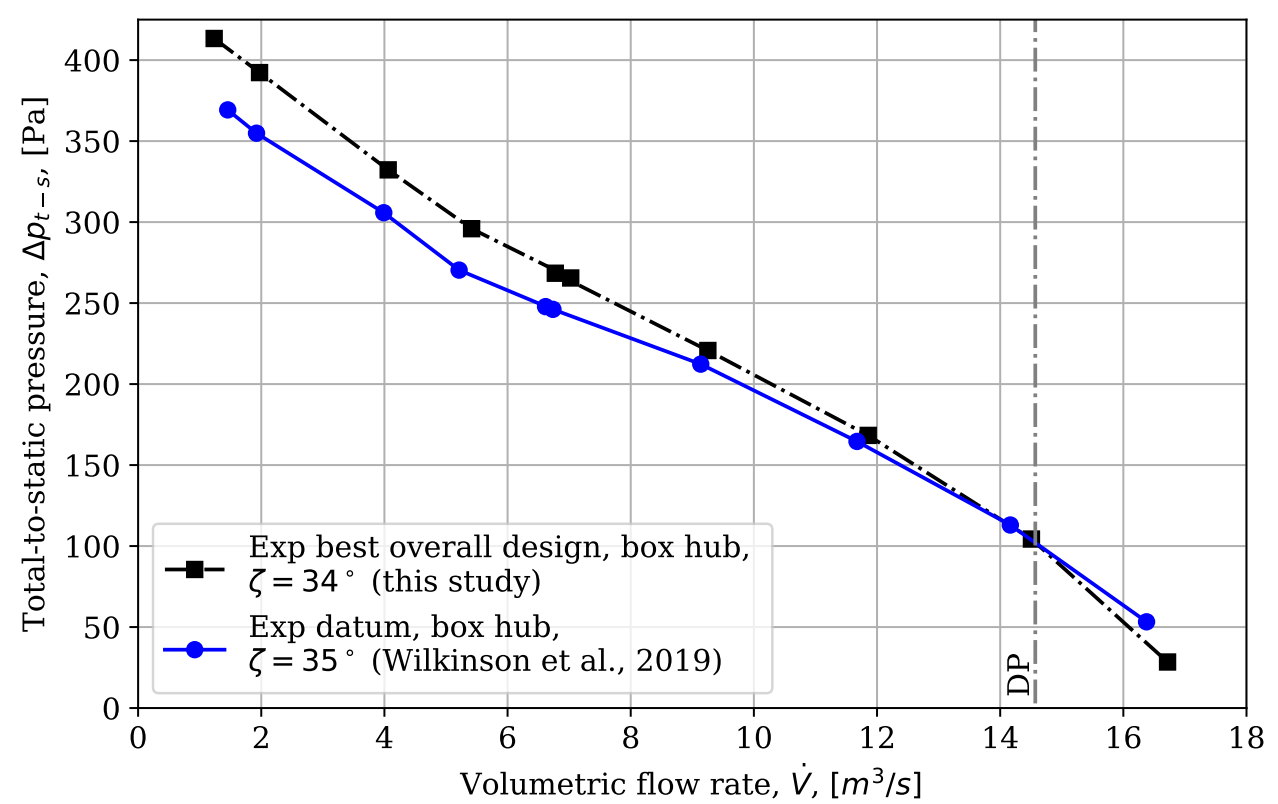

Figure 10.8: Experimental total-to-static pressure rise comparison between increasing blade stagger angle and optimized TE end-plate design 


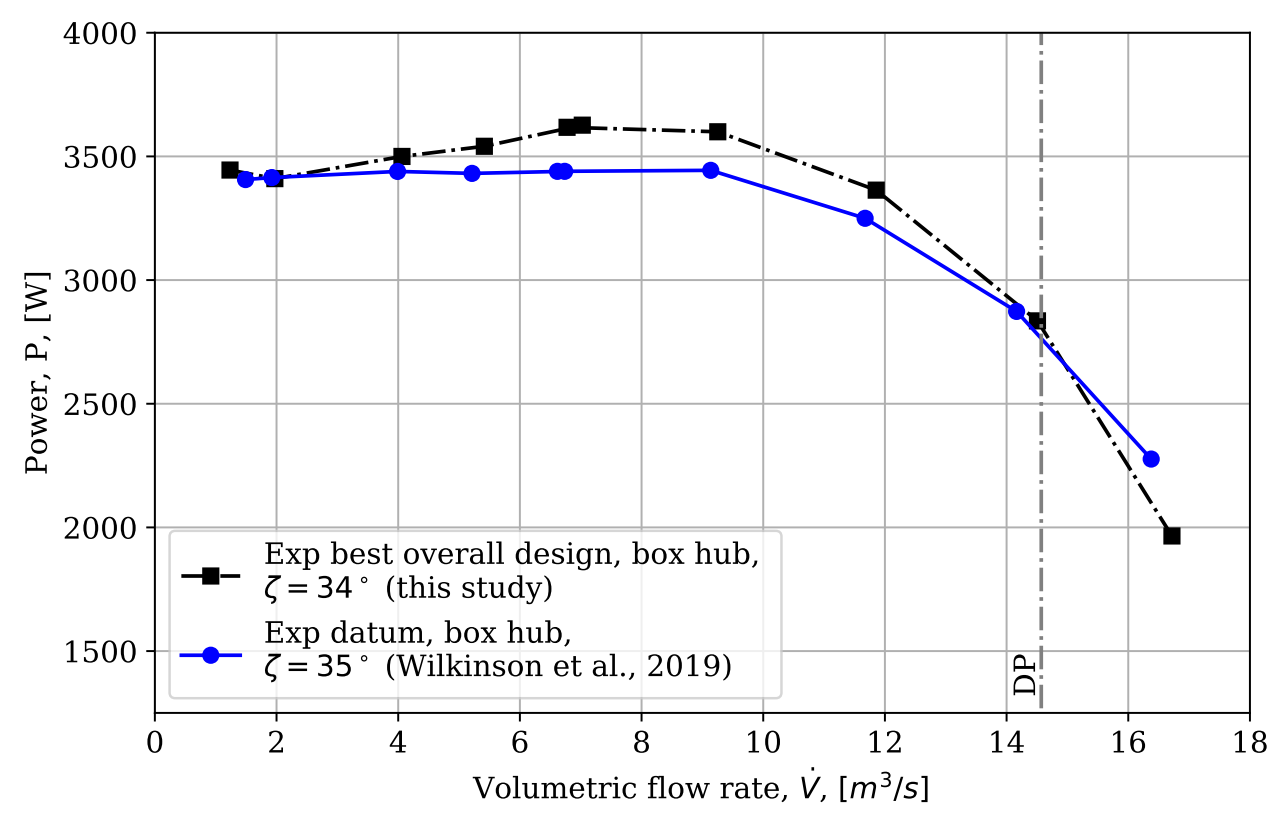

Figure 10.9: Experimental power comparison between increasing blade stagger angle and optimized TE end-plate design

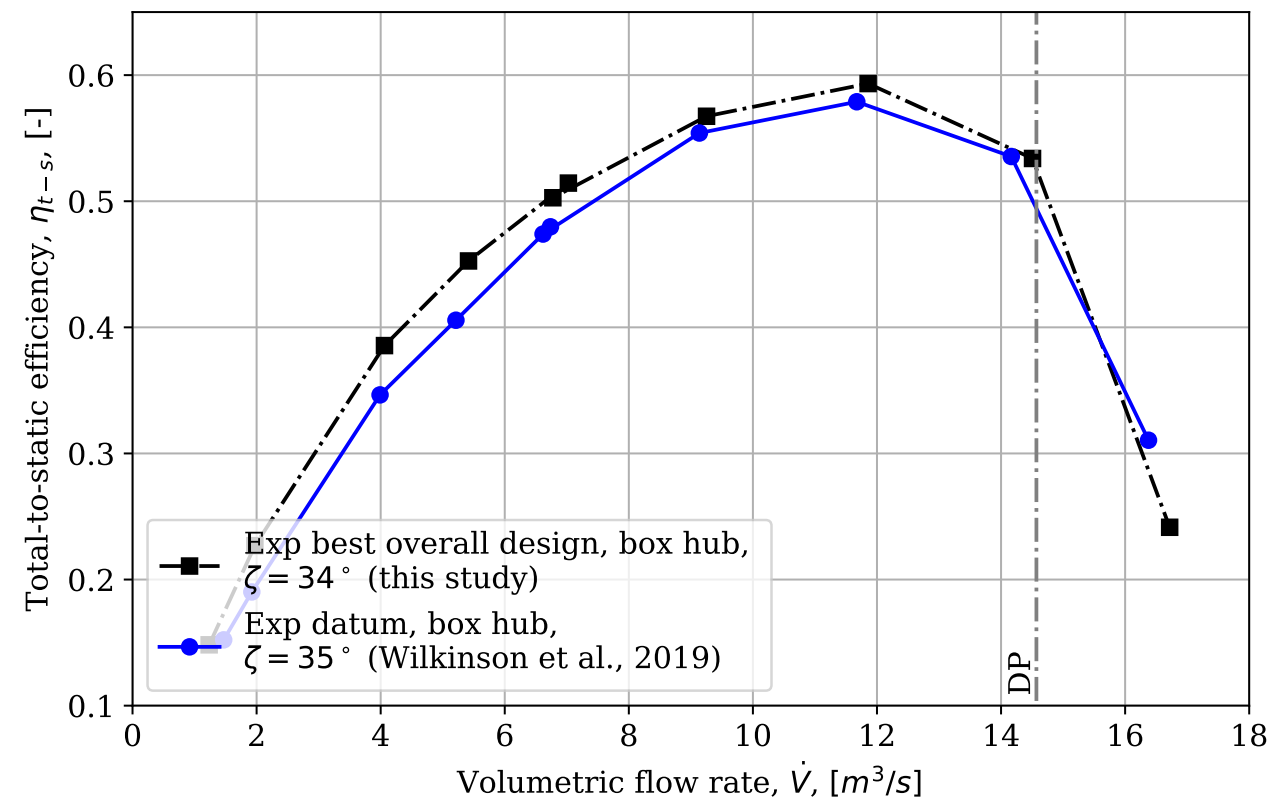

Figure 10.10: Experimental total-to-static efficiency comparison between increasing blade stagger angle and optimized TE end-plate design 
As evident in the figures above, the optimized TE end-plate design is found to perform more consistently compared to increasing the blade's stagger angle. The optimized end-plate design is found to have superior total-to-static pressure rise characteristics across the majority of the fan's operational envelope. Comparing the total-to-static pressure rise differences between each case and the fan datum, an average gain of 62.66 percent more is found with the optimized design compared to increasing the blade's stagger angle. Moreover, these improvements are further augmented towards the rotor stall margin. However, this is met with a greater power requirement across the majority of the flow rates.

Despite this increase in power requirement, the optimized end-plate design is found to improve the fan's total-to-static efficiency characteristic across the majority of the fan's operational envelope. Additionally, the optimized design is found to perform better at below design point flow rates compared to increasing the blade's stagger angle. These findings suggest the blade tip to be crucial in improving the fan's various performance metrics.

\subsubsection{Verification of results}

In order to be able to substantiate such conclusions, it is also important to check that the quoted performance improvements are greater than the test facility's measurement error and that the uncertainty errors are within an acceptable range. As such, the current section assesses the experimental results of the tested endplate designs and fan datum with respect to its bias and variation.

\section{Variation}

As previously explained, the test facility used in the experimental evaluations of the end-plate designs follows the ISO 5801, type A test set-up. As such, using the root-sum-square (RSS) method for each fan characteristics (as described in the ISO 5801 standard), the experimental results for each tested design are presented in Table 10.3 in the form of a maximum uncertainty value. The maximum allowable uncertainty of results, likewise defined in ISO 5801, for each respective parameter is also included for comparison. 
Table 10.3: Maximum experimental test measurement uncertainty (valid for $\left.4.12 \mathrm{~m}^{3} / \mathrm{s}<\dot{V}<16.38 \mathrm{~m}^{3} / \mathrm{s}\right)$

\begin{tabular}{ccccc}
\hline Design & \multicolumn{4}{c}{ Variable } \\
& $\dot{\boldsymbol{V}}[\%]$ & $\boldsymbol{\Delta}_{\boldsymbol{t}-\boldsymbol{s}}[\%]$ & Power [\%] & $\boldsymbol{\eta}_{\boldsymbol{t}-\boldsymbol{s}}[\%]$ \\
\hline Best overall & 0.74 & 1.99 & 0.95 & 0.73 \\
Modified & 0.83 & 3.08 & 1.99 & 0.80 \\
Fan datum & 0.72 & 2.21 & 1.20 & 0.85 \\
Maximum (ISO 5801) & 2.0 & 1.4 & 2.5 & 3.2 \\
\hline
\end{tabular}

It should be noted that poor repeatability of the results was obtained during testing when measuring at volumetric flow rates below $4.12 \mathrm{~m}^{3} / \mathrm{s}$ and above $16.38 \mathrm{~m}^{3} / \mathrm{s}$. This is more than likely a result of low reading measurement errors and flow instabilities near stall, leading to a magnification of the uncertainty information. Consequently, the included values only represent the results taken between $4.12 \mathrm{~m}^{3} / \mathrm{s}$ $<\dot{V}<16.38 \mathrm{~m}^{3} / \mathrm{s}$.

Comparing the $\Delta p_{t-s}$ performance characteristic errors of the various designs to the maximum allowable value, it is evident than none of the designs fall below the maximum limit. This indicates an unacceptable uncertainty level with the confidence interval being less than 95\% (International Organization for Standardization, 2007). When comparing the remaining performance metrics, all are found to lie sufficiently below the maximum. Therefore, only these characteristic variables can be referred to with confidence. However, as the reported total-to-static pressure rise values for the best overall design are significantly larger than the fan datum over the entire operational envelope, these error values are deemed acceptable when comparing the two designs.

\section{Bias}

In order to verify that the quoted performance improvements are greater than the test facility's measurement error, a comparison is made to the differences in experimental results between the various end-plate designs and the fan datum at the fan's design point flow rate. Table 10.4 gives the results with the test facility's maximum instrumentation error (as introduced in Section 10.2.2 included for comparison. 
Table 10.4: Maximum experimental test measurement error vs end-plate design performance improvement over fan datum

\begin{tabular}{ccc}
\hline Design & \multicolumn{2}{c}{ Variable } \\
& $\boldsymbol{\Delta} \boldsymbol{p}[\%]$ & $\boldsymbol{\Delta}$ Torque $[\%]$ \\
\hline Best overall & 7.86 & 10.17 \\
Modified & 2.00 & 1.66 \\
Maximum measurement error (from Table 10.1$)$ & 0.36 & 1.10 \\
\hline
\end{tabular}

As evident, all maximum measurement error values fall well below the various end-plate design performance improvement margins. This suggests that the test facility's instrumentation is more than adequate to capture the performance improvements of the end-plate designs. 


\section{Chapter 11}

\section{Conclusion}

The following chapter summarizes the objectives and methodology used throughout the current study. The key findings and future work recommendations are also included.

\subsection{Objectives and methodology}

The present study conducts a numerical investigation into tip clearance effects specific to axial flow fan performance characteristics. The main aim of the study is to gain understanding into the relevant leakage flow phenomena present near the blade tip. This in order to develop a method by which the effective mitigation of such flow phenomena can be implemented in order to improve fan performance characteristics. This is investigated by means of a numerical approach to determine the feasibility of the design strategy.

The effect of varying the fan's tip clearance on fan performance characteristics is numerically investigated. The findings, along with relevant literature, are used to identify two predominant leakage flow phenomena for control. Consequently, two design strategies are implemented to mitigate the detrimental effects of the identified flow phenomena and determine their effect on the fan's performance characteristics.

Of the two introduced designs, the most promising design is chosen for optimization based on their numerical performance results. The most promising design is optimized based on a multi-objective multi-point (MOMP) optimization process in order to best improve the fan's many performance characteristics. Finally, once optimized, the best competing designs are experimentally tested in order to verify the numerical results and draw conclusions. 


\subsection{Key findings}

The key findings of the current study are herewith enumerated:

1. Increased tip clearance in an axial flow fan is associated with having a detrimental effect on fan performance. Its reduction is found to increase both fan total-to-static pressure rise and fan total-to-static efficiency characteristics across the entire stable operating range. A large pressure differential present near the blade tip induces a localized flow, known as tip leakage flow (TLF), through the tip gap. The TLF exits on the blade's suction side and merges with the main flow, thereby causing the formation of a tip leakage vortex (TLV). The formation and subsequent bursting of the TLV is thought to incur greater mixing losses within the rotor annulus owing to a decrease in fan performance. Various methods employed within literature for the passive control of TLVs are investigated.

2. Through consideration of available open-source meshing software, a semiautomated hybrid meshing strategy is introduced in Chapter 3 in order to mesh the P3DM domain of the datum fan. Using a $1 / 8^{\text {th }}$ annular rotor section, the domain is meshed by first creating a baseline mesh to which a bottom up approach is used to prepare and create the required wall layer cells. The results of the proposed meshing strategy and numerical set-up are found to correlate well with experimental data and the differences are comparable to that found in literature. This demonstrates the validity in using the proposed meshing strategy.

3. Helicity contour plots are used in the visualization and quantification of the TLV of the datum fan in Chapter 5. The findings indicate the presence of two clockwise rotating vortex structures of differing size and intensity on the blade pressure and blade suction side, denoted TLV/PS and TLV/SS respectively. Upon further analysis, it is found that the inception of the TLV/SS on the blade's suction side further develops downstream to which it matures into the TLV/PS on the aft blade's pressure side. The merging of these two vortex structures and subsequent blade-to-blade vortex interaction affects the relative flow field within the vicinity of the tip region. To quantify the TLV/SS, a TLF velocity and TLV exit trajectory angle variable is introduced. A reduction in the TLF velocity and TLV exit trajectory angle is evident with a reduction in tip clearance. This is found to increase the chordwise blade loading distribution near the blade tip and subsequently improve all fan performance characteristics across the fan's entire stable operating range. 
4. According to Corsini et al. (2006), a local thickening of the blade tip on the blade's pressure side reduces the TLF velocity within the tip region and improves the efficiency characteristics of a subsonic axial flow fan. As the rationale behind this design is aligned with that of the desired flow phenomena to be controlled in this study, Section 6.2 introduces three constant thickness (CT) end-plate design concepts which are analysed numerically to determine their effect on fan performance. All pressure side end-plate designs indicate improved performance characteristics at lower than design point flow rates with an increased rotor stall efficiency plateau. The suction side end-plate design is found only to increase the fan performance characteristics at the higher than design point evaluated flow rates. All designs are found to reduce the TLF within the tip region at the fan's design point flow rate; however, none are found to improve the fan's performance characteristics at this operating point. The findings suggest that an operating point specific CT end-plate thickness exists whereby effective control of the TLV can be implemented over a specific beneficial operating range.

5. Through introduction of a novel trailing edge (TE) end-plate concept, presented in Section 6.3, the control and mitigation of the TLV/SS exit trajectory angle is implemented. Using flat plate theory to reduce the inherit complexity of the system, the end-plate setting angle and dimensions are chosen to determine an initial operating range for control of the TLV. The findings indicate improved fan performance characteristics across all evaluated flow rates, including that of the design point flow rate. The new design is found to control the TLV through a reduction of the TLV/SS exit trajectory angle and subsequent reduction of the blade-to-blade vortex interaction. Additionally, the design is found to shift the blade's peak loading distribution further aft along the blade tip. This has been described to have a beneficial effect on the mixing of the TLF and subsequent TLV formation.

6. As a result of the above, the TE end-plate design is chosen for numerical optimization. Through screening of the design (presented in Chapter 7), a multi-objective multi-point (MOMP) objective function is introduced with the aim of best compromising between improving the fan's performance characteristics at both design point and below design point flow rates. This stems from the relevant literature indicating the adverse effects of wind on the performance characteristics of ACHEs. Finally, a two-level full factorial design is used to determine the most influential end-plate parameters on fan performance for exploitation in the optimization procedure. This analysis also reveals the upper dimensions for the various end-plate parameters.

7. Using a multivariate extension of the traditional Latin hypercube sampling 
method, the TE end-plate design space is initially populated. This method was selected as it was found to offer significant advantages over many other competing alternatives. The database is then optimized using the Design and Analysis of Computer aided Experiments (DACE) surrogate model (SM) of Sacks et al. (1989) with the accompanying Efficient Global Optimization (EGO) algorithm of Jones et al. (1998). Due to large simulation times encountered during the datum fan analysis, this surrogate model was selected with aim of reducing the required number of function evaluations. However, likewise with many SM, the DACE model consists of fundamental issues when estimating the optimal model hyperparameters for fitting of the response surface. Various methods are employed in this investigation to compensate for these inadequacies. Additionally, as this model is a derivative of the well known Kriging model, it provides an estimation for its own uncertainty. This is a highly attractive feature as verification of the models error can easily be performed. Nonetheless, additional verification of the SM is performed using a set of well known benchmark test functions.

8. Chapter 9 presents the numerical results of the TE end-plate optimization process using the aforementioned DACE model and EGO algorithm combination. Validation of the initial database is carried out to ensure sufficient reduction of the model's error and uncertainty. Thereafter, algorithm convergence is checked to ensure that the general region of optimality is reached. As this algorithm employs a two step optimization search, which systematically refines the search space from a more global search criterion to that of a local one, sufficiency of the former is easily accomplished by ensuring a reduction in explorative points. Finally, the Pareto-front of non-dominated solutions is introduced with the aim of outlining competing designs. As evident, a local clustering of points on the front is synonymous with the location of the optimal solution. As such, this design is selected for experimental testing. Additionally, a modified version of this design is selected with the aim of improving the fan's performance at lower volumetric flow rates.

9. Finally, the experimental method is presented in Chapter 10. The TE endplate design's construction is presented with a step-by-step explanation. A brief discussion into the fan test facility and various testing equipment is also presented, with an overview of the equipment's measurement errors. Thereafter, an explanation of the fan testing procedures and test set-up is given. The experimental results of the optimized end-plate design indicate improved fan performance characteristics at greater than peak efficiency flow rates. This effect is compared to increasing the blade's stagger angle with the findings indicating superior performance across the majority of the fan's 
operational envelope for the optimized design. The modified version of this design is found to have similar performance characteristics when considering the shift in the fan's operating point due to increased pressure losses. This is significant as this design is better able to improve the fan's performance characteristics at higher than peak efficiency flow rates with an almost negligible performance loss at lower volumetric flow rates.

\subsection{Recommended future work}

Future work and additional recommendations are proposed based on the results of the current study.

- Although the newly implemented meshing strategy in the current study is found to sufficiently validate against the experimental data, the method can still be further improved. Difficulties in obtaining a sufficient number of wall layer cells over surface patches with a sufficiently low maximum cell volume ratio leads to possible uncertainty in the solution's interpolation within these regions. To alleviate this problem, alteration of the various meshing programs source code would be required to be able to adapt current meshes in order to externally add cells within a set geometric domain. This would allow growth of the boundary layer cells sufficiently far away from the surface patches to which the program would then be able to mesh the remainder of the domain.

- As discussed in Section 10.3, a direct relationship exists between the TE endplate's setting angle and the fan's performance characteristics at a specific volumetric flow rate. As this design was optimized for the M-fan, which has a greater than peak efficiency design point flow rate, the optimized design was found to have a relatively large end-plate setting angle. Consequently, this correlates to reduced performance characteristics at below peak efficiency flow rates. As such, optimization of this design should be performed at the fan's peak efficiency operating point to determine the potential of improving the performance at this point.

- As the current end-plate optimization is rudimentary in its control variable parameterization, further optimization should be performed by introducing more control variables. This would allow the algorithm to gain more control in the design's shape, thereby possibly determining a more optimal design. However, this would come at a significantly increased computational expense which should be considered before its practical implementation. 


\section{References}

Adamczyk, J., Celestina, M. and Greitzer, E. (1993). The role of tip clearance in high-speed fan stall. ASME Journal of Turbomachinery, vol. 115, pp. 28-38.

Ali, M.M., Khompatraporn, C. and Zabinsky, Z.B. (2005). A numerical evaluation of several stochastic algorithms on selected continuous global optimization test problems. Journal of global optimization, vol. 31, no. 4, pp. 635-672.

Ali, M.M. and Törn, A. (2004). Population set-based global optimization algorithms: some modifications and numerical studies. Computers $\&$ Operations Research, vol. 31, no. 10, pp. 1703-1725.

Andrianakis, I. and Challenor, P.G. (2012). The effect of the nugget on gaussian process emulators of computer models. Computational Statistics \& Data Analysis, vol. 56, no. 12, pp. 4215-4228.

Ashihara, K. and Goto, A. (2001). Turbomachinery blade design using 3-d inverse design method, cfd and optimization algorithm. In: ASME Turbo Expo 2001: Power for Land, Sea, and Air. American Society of Mechanical Engineers Digital Collection.

Bagheri, S., Konen, W. and Bäck, T. (2017). Comparing kriging and radial basis function surrogates. In: Proc. 2\%. Workshop Computational Intelligence, pp. 243-259.

Benham, P.P., Crawford, R.J. and Armstrong, C.G. (1996). Mechanics of Engineering Materials. 2nd edn. Longman Group, Harlow, Essex, England. ISBN 978-0-582-25164-9.

Bergh, J. (2018). On the evaluation of common design metrics for the optimization of non-axisymmetric endwall contours for a 1-stage turbine rotor. Ph.D. thesis, University of Cape Town.

Chen, B. and Yuan, X. (2008). Advanced aerodynamic optimization system for turbomachinery. Journal of turbomachinery, vol. 130, no. 2. 
Cheney, E.W. and Kincaid, D.R. (2012). Numerical mathematics and computing.

Corsini, A., Delibra, G., Rispoli, F. and Sheard, A. (2015). Aeroacoustic assessment of leading edge bumps in industrial fans. In: Proceedings of the International Conference on Fan Noise, Technology and Numerical Methods 2015. Inst. of Mechanical Engineers London.

Corsini, A., Rispoli, F. and Sheard, A.G. (2006). Development of improved blade tip end-plate concepts for low-noise operation in industrial fans. In: Conference on Modelling Fluid Flow (CMFF'06) The 13th International Conference on Fluid Flow Technologies Budapest, Hungary.

Corsini, A., Rispoli, F. and Sheard, A.G. (2007b). Development of improved blade tip end-plate concepts for low-noise operation in industrial fans. Proceedings of the Institution of Mechanical Engineers, Part A: Journal of Power and Energy, vol. 221, no. 5, pp. 669-681. ISSN 09576509.

Corsini, A., Rispoli, F. and Sheard, A.G. (2010). Shaping of tip end-plate to control leakage vortex swirl in axial flow fans. Journal of Turbomachinery, vol. 132, no. 3, p. 031005. ISSN 0889504X.

Corsini, A. and Sheard, A.G. (2007a). Tip end-plate concept based on leakage vortex rotation number control. Journal of Computational and Applied Mechanics, vol. 8 , no. 1 , pp. 21-37.

Corsini, A. and Sheard, A.G. (2013). End-plate for noise-by-flow control in axial fans. Periodica Polytechnica Mechanical Engineering, vol. 57, no. 2, pp. 3-16. ISSN 1587379X.

Crombecq, K., Couckuyt, I., Gorissen, D. and Dhaene, T. (2009). Space-filling sequential design strategies for adaptive surrogate modelling. In: The first international conference on soft computing technology in civil, structural and environmental engineering, vol. 38.

Crombecq, K., Laermans, E. and Dhaene, T. (2011). Efficient space-filling and noncollapsing sequential design strategies for simulation-based modeling. European Journal of Operational Research, vol. 214, no. 3, pp. 683-696.

Cumpsty, N.A. (2004). Compressor Aerodynamics. Krieger Pub., 2004. ISBN $1575242478,9781575242477$.

Cumpsty, N.A. and Storer, J.A. (1991). Tip leakage flow in axial compressors. Journal of Turbomachinery, vol. 113, no. 2, pp. 252-259. 
De Maesschalck, C., Lavagnoli, S., Paniagua, G., Verstraete, T., Olive, R. and Picot, P. (2016). Heterogeneous optimization strategies for carved and squealerlike turbine blade tips. Journal of Turbomachinery, vol. 138, no. 12.

Deng, X., Guo, F., Liu, Y. and Han, P. (2013). Aero-mechanical optimization design of a transonic fan blade. In: ASME Turbo Expo 2013: Turbine Technical Conference and Exposition.

Denton, J.D. (1993). Loss mechanisms in turbomachines. ASME International Gas Turbine and Aeroengine Congress and Exposition, pp. 19-21.

Deutsch, J.L. and Deutsch, C.V. (2012). Latin hypercube sampling with multidimensional uniformity. Journal of Statistical Planning and Inference, vol. 142, no. 3 , pp. $763-772$.

Durantin, C., Marzat, J. and Balesdent, M. (2016). Analysis of multi-objective kriging-based methods for constrained global optimization. Computational Optimization and Applications, vol. 63, no. 3, pp. 903-926.

Endress and Hauser Group (2020). Differential pressure Deltabar PMD75. https://www.za.endress.com/en/field-instruments-overview/ pressure/Differential-Pressure-Deltabar-PMD75. Accessed: 28 October 2020 .

Everitt, B. (2012). Introduction to optimization methods and their application in statistics. Springer Science \& Business Media.

Flannery, B.P., Press, W.H., Teukolsky, S.A. and Vetterling, W. (1992). Numerical recipes in c. Press Syndicate of the University of Cambridge, New York, vol. 24, p. 78 .

Forrester, A.I., Bressloff, N.W. and Keane, A.J. (2006). Optimization using surrogate models and partially converged computational fluid dynamics simulations. Proceedings of the Royal Society A: Mathematical, Physical and Engineering Sciences, vol. 462, no. 2071, pp. 2177-2204.

Garg, A. and Leibovich, S. (1979). Spectral characteristics of vortex breakdown flow fields. The Physics of Fluids, vol. 22, no. 11, pp. 2053-2064.

Giuni, M. (2013). Formation and early development of wingtip vortices. Ph.D. thesis, Department of Aerospace Engineering, University of Glasgow, Scotland.

Goinis, G., Voß, C. and Aulich, M. (2013). Automated optimization of an axialslot type casing treatment for a transonic compressor. In: Turbo Expo: Power for Land, Sea, and Air, vol. 55232, p. V06BT43A011. 
Green, S.I. (1995). Fluid Vortices: Fluid Mechanics and Its Applications, in: Particle Interactions with Vortices. Kluwer Academic Publisher.

Haupt, R.L. and Ellen Haupt, S. (2004). Practical genetic algorithms. Wiley Online Library.

Inoue, M. and Furukawa, M. (2002). Physics of Tip Clearance Flow in Turbomachinery. vol. 2: Symposia and General Papers, Parts A and B of Fluids Engineering Division Summer Meeting, pp. 777-789.

Inoue, M. and Kuroumaru, M. (1989). Structure of Tip Clearance Flow in an Isolated Axial Compressor Rotor. ASME Journal of Turbomachinery, vol. 111, no. 3 , pp. 250-256.

Inoue, M., Kuroumaru, M. and Furukawa, M. (1986). Behavior of tip leakage flow behind an axial compressor rotor. ASME Journal of Gas Turbine and Power, 108, vol. 108, no. 1 , pp. $7-14$.

Jamil, M. and Yang, X.-S. (2013). A literature survey of benchmark functions for global optimisation problems. International Journal of Mathematical Modelling and Numerical Optimisation, vol. 4, no. 2, pp. 150-194.

Jeong, S., Minemura, Y. and Obayashi, S. (2006). Optimization of combustion chamber for diesel engine using kriging model. Journal of fluid Science and Technology, vol. 1, no. 2, pp. 138-146.

Jeong, S. and Obayashi, S. (2005). Efficient global optimization (ego) for multiobjective problem and data mining. In: 2005 IEEE congress on evolutionary computation, vol. 3, pp. 2138-2145.

Jiang, D., Luo, H. and Zhang, X. (2015). Numerical study of the leakage flow on a novel turbine blade tip. Procedia Engineering, vol. 99, pp. 413-422.

Jones, D.R., Schonlau, M. and Welch, W.J. (1998). Efficient global optimization of expensive black-box functions. Journal of Global optimization, vol. 13, no. 4, pp. $455-492$.

Juretić, F. (2015). cfMesh v1.1 User Guide. http://cfmesh.com/wp-content/ uploads/2015/09/User_Guide-cfMesh_v1.1.pdf . Accessed: 3 June 2018.

Kim, J., Kim, J. and Kim, K. (2011). Axial-flow ventilation fan design through multi-objective optimization to enhance aerodynamic performance. Journal of fluids engineering, vol. 133, no. 10. 
Kjellstrom, G. and Taxen, L. (1981). Stochastic optimization in system design. IEEE Transactions on Circuits and Systems, vol. 28, no. 7, pp. 702-715.

Kok, S. (2012). The asymptotic behaviour of the maximum likelihood function of kriging approximations using the gaussian correlation function.

Kröger, D.G. (2004). Air-Cooled Heat Exchangers and Cooling Towers - ThermalFlow Performance Evaluation and Design, vol. 1. PennWell Books. ISBN 1593700199.

Lakshminarayana, B. (1970). Methods of predicting the tip clearance effects in axial flow turbomachinery. Journal of Basic Engineering, vol. 92, no. 3, pp. 467-480. ISSN 0098-2202.

Lampinen, J. (2002). A constraint handling approach for the differential evolution algorithm. In: Proceedings of the 2002 Congress on Evolutionary Computation. CEC'02 (Cat. No. 02TH8600), vol. 2, pp. 1468-1473.

Launder, B. and Spalding, D. (1974). The numerical computation of turbulent flows. Computer methods in applied mechanics and engineering, vol. 3, no. 2, pp. 269-289.

Li, X., Liu, Z. and Lin, Y. (2017). Multipoint and multiobjective optimization of a centrifugal compressor impeller based on genetic algorithm. Mathematical Problems in Engineering, vol. 2017.

Liu, H., Xu, S. and Wang, X. (2016). Sampling strategies and metamodeling techniques for engineering design: comparison and application. In: ASME Turbo Expo 2016: Turbomachinery technical conference and exposition.

Lophaven, S.N., Nielsen, H.B. and Søndergaard, J. (2002). Aspects of the matlab toolbox DACE. Citeseer.

Louw, F.G. (2015). Investigation of the flow field in the vicinity of an axial flow fan during low flow rates. $\mathrm{PhD}$, Department of Mechanical and Mechatronic Engineering, Stellenbosch University, South Africa.

Martin, J.D. and Simpson, T.W. (2004). On the use of kriging models to approximate deterministic computer models, international design engineering technical conferences and computers and information in engineering conference, utah usa. vol. 4.

Menter, F., Kuntz, M. and Langtry, R. (2003). Ten years of industrial experience with the sst turbulence model. Heat and Mass Transfer, vol. 4. 
MinWaterCSP (2017). https://www.minwatercsp.eu/. Accessed: 5 May 2018.

Mitchell, A.M. and Delery, J. (2001). Research into vortex breakdown control. Progress in Aerospace Sciences, vol. 37, pp. 385-418.

Mockus, J., Tiesis, V. and Zilinskas, A. (1978). The application of bayesian methods for seeking the extremum. Towards global optimization, vol. 2, no. 117-129, p. 2.

Mueller, L., Alsalihi, Z. and Verstraete, T. (2013). Multidisciplinary optimization of a turbocharger radial turbine. Journal of turbomachinery, vol. 135, no. 2.

OpenCFD Ltd (2018). OpenFOAM user guide. https://www.openfoam.com/ documentation/user-guide/index.php. Accessed: 12 June 2018.

Pei, J., Wang, W. and Yuan, S. (2016). Multi-point optimization on meridional shape of a centrifugal pump impeller for performance improvement. Journal of Mechanical Science and Technology, vol. 30, no. 11, pp. 4949-4960.

Peng, C.-Y. and Wu, C.J. (2014). On the choice of nugget in kriging modeling for deterministic computer experiments. Journal of Computational and Graphical Statistics, vol. 23, no. 1, pp. 151-168.

Pepelyshev, A. (2010). The role of the nugget term in the gaussian process method. In: mODa 9-Advances in Model-Oriented Design and Analysis, pp. 149-156.

Price, K., Storn, R.M. and Lampinen, J.A. (2005). Differential evolution: a practical approach to global optimization. Springer Science \& Business Media.

Rains, D.A. (1954). Tip clearance flows in axial compressors and pumps. Ph.D. thesis, California Institute of Technology, Pasadena, California.

Ruden, P. (1944). Investigation of single stage axial fans.

Sacks, J., Welch, W.J., Mitchell, T.J. and Wynn, H.P. (1989). Design and analysis of computer experiments. Statistical science, pp. 409-423.

Sasena, M.J., Papalambros, P. and Goovaerts, P. (2002). Exploration of metamodeling sampling criteria for constrained global optimization. Engineering optimization, vol. 34, no. 3, pp. 263-278.

Song, H., Choi, K. and Lamb, D. (2013). A study on improving the accuracy of kriging models by using correlation model/mean structure selection and penalized log-likelihood function. 
Spall, R.E., Gatski, T.B. and Grosch, C.E. (1987). A criterion for vortex breakdown. The Physics of fluids, vol. 30, no. 11, 1987, pp. 3434-3440. ISSN 00319171.

Storer, J.A. (1991). Tip clearance flow in axial compressors. Ph.D. thesis, Department of Engineering, University of Cambridge, United Kingdom.

Storn, R. and Price, K. (1997). Differential evolution-a simple and efficient heuristic for global optimization over continuous spaces. Journal of global optimization, vol. 11, no. 4, pp. 341-359.

Sudhoff, S. (2007). Multi-objective optimization, lecture notes, ece 630 special topics. Purdue University.

International Organization for Standardization (2007). ISO 5801:2007 industrial fans - performance testing using standardized airways.

The OpenFOAM Foundation (2014). Non-Conforming AMI Patches. https: //openfoam.org/release/2-3-0/non-conforming-ami/. Accessed: 22 Feb. 2019 .

Tiralap, A., Tan, C.S., Donahoo, E., Montgomery, M. and Cornelius, C. (2017). Effects of rotor tip blade loading variation on compressor stage performance. Journal of Turbomachinery, vol. 139, no. 5, p. 051006. ISSN 0889-504X.

Uchida, S., Nakamura, Y. and Ohsawa, M. (1985). Experiments on the axisymmetric vortex breakdown in a swirling air flow. Japan Society of Aeronautical Space Sciences Transactions, vol. 27, pp. 206-216.

Vad, J. (2008). Aerodynamic effects of blade sweep and skew in low-speed axial flow rotors at the design flow rate: an overview. Proceedings of the Institution of Mechanical Engineers, Part A: Journal of Power and Energy, vol. 222, no. 1, pp. 69-85.

van der spuy, S.J. (2011). Perimeter fan performance in forced draught air-cooled steam condensers. Ph.D. thesis, Stellenbosch University.

Venter, S. and Kröger, D. (1992). The effect of tip clearance on the performance of an axial flow fan. Energy conversion and management, vol. 33, no. 2, pp. 89-97.

Venter, S.J. and Kroger, D. (1990). The effectiveness of axial flow fans in A-frame plenums. Ph.D. thesis, Stellenbosch: University of Stellenbosch.

Verein Deutscher Ingenieure (1966). Vdi-richtlinien-abnahme- und leistungsversuche an ventilatoren vdi 2044 , berlin. 
Volino, R.J. (2014). Effects of endwall boundary layer thickness and blade tip geometry on flow through high pressure turbine passages. In: ASME Turbo Expo 2014: Turbine Technical Conference and Exposition. American Society of Mechanical Engineers Digital Collection.

Walters, D.K. and Cokljat, D. (2008). A three-equation eddy-viscosity model for reynolds-averaged navier-stokes simulations of transitional flow. Journal of fluids engineering, vol. 130, no. 12, p. 121401.

Watson, A.G. and Barnes, R.J. (1995). Infill sampling criteria to locate extremes. Mathematical Geology, vol. 27, no. 5, pp. 589-608.

Wilkinson, M., Thomas, K. and van der Spuy, S.J. (2018). MinWaterCSP: Minimized water consumption in CSP plants. Tech. Rep. 654443.

Wilkinson, M.B. (2017). The Design of an Axial Flow Fan for Air-Cooled Heat Exchanger Applications. Master's thesis, Department of Mechanical and Mechatronic Engineering, Stellenbosch University, South Africa.

Wilkinson, M.B., van der Spuy, S.J. and von Backström, T.W. (2019). Performance testing of an axial flow fan designed for air-cooled heat exchanger applications. Journal of Engineering for Gas Turbines and Power, vol. 141, no. 5.

Wolf Dynamics (2013). Tips and tricks in OpenFOAM. http://www. wolfdynamics.com/wiki/OFtipsandtricks.pdf. Accessed: 10 Feb. 2019.

Wolpert, D. and Macready, W. (1997). No free lunch theorems for optimization. IEEE transactions on evolutionary computation, vol. 1, no. 1, pp. 67-82.

Ye, X., Li, P., Li, C. and Ding, X. (2015). Numerical investigation of blade tip grooving effect on performance and dynamics of an axial flow fan. Energy, vol. 82 , pp. 556-569.

Ye, X., Zhang, J. and Li, C. (2017). Effect of blade tip pattern on performance of a twin-stage variable-pitch axial fan. Energy, vol. 126, pp. 535-563.

Zhang, L., Jin, Y. and Jin, Y. (2014). Effect of tip flange on tip leakage flow of small axial flow fans. Journal of Thermal Science, vol. 23, no. 1, pp. 45-52. 


\section{Appendix A}

\section{Fan scaling}

This section defines the procedure used to determine the scaling of the M-fan (details given in Section 1.2). This is in order to be able to make comparisons between scale numerical and experimental results. Table A.1 shows the fan specifications of design and model scale (as determined by Wilkinson et al., 2019).

Table A.1: Fan specifications (Wilkinson et al., 2019)

\begin{tabular}{ccc}
\hline & Design & Scale \\
\hline Diameter & $24 \mathrm{ft}[7.315 \mathrm{~m}]$ & $1.534 \mathrm{~m}$ \\
Flow rate & $333 \mathrm{~m}^{3} / \mathrm{s}$ & $14.37 \mathrm{~m}^{3} / \mathrm{s}$ \\
Rotational speed & $151 \mathrm{rpm}$ & $722 \mathrm{rpm}$ \\
\hline
\end{tabular}

As shown, a single design specification is given for the scale M-fan. However, when varying the tip clearance, as done during experimental testing, consideration of the fan diameter must be taken into account as this does not remain constant.

When scaling the fan, the design specifications remain constant while the scale model specifications are defined to suit the available test facility set-up. However, when varying the tip clearance during experimental testing, the casing diameter of the test facility remains fixed while the blade tip diameter is altered to achieve the desired tip clearance. This means that the scaling factor, and hence the scale fan's design point flow rate, changes when varying the tip clearance.

The equation used to scale the volumetric flow rate of the design fan is defined as:

$$
\begin{aligned}
\dot{V}_{m} & =\dot{V}_{d}(\lambda)^{3}\left(N_{m} / N_{d}\right) \\
\lambda & =D_{m} / D_{d}
\end{aligned}
$$


where $\mathrm{m}$ and $\mathrm{d}$ represent the model and design fan, respectively, and $\lambda$ represents the geometric scaling factor. Using this, the model fan diameter is calculated by subtracting the desired tip clearance from the fan casing diameter (measured to be $1.542 \mathrm{~m}$ at Stellenbosch University's fan test facility). Using the values from Table A.1 and substituting these into Equation A.1, the various design point flow rates and geometric scaling factors for the scale $\mathrm{M}$-fan at various tip clearances are given in Table A.2.

Table A.2: Scale M-fan specifications for various tip clearances [ $\left.N_{m}=722 \mathrm{rpm}\right]$

\begin{tabular}{ccc}
\hline Tip clearance & Diameter $[\mathrm{m}]$ & Flow rate $\left[\mathrm{m}^{3} / \mathrm{s}\right]$ \\
\hline $2 \mathrm{~mm}$ & 1.538 & 14.80 \\
$4 \mathrm{~mm}$ & 1.534 & 14.68 \\
$6 \mathrm{~mm}$ & 1.530 & 14.57 \\
\hline
\end{tabular}

The final design specifications for the scale M-fan are given in Table A.3.

Table A.3: Scale M-fan design specifications

\begin{tabular}{ll}
\hline Design specifications & \\
\hline Number of blades [-] & 8 \\
Blade setting angle [] & 34 \\
$D_{\text {rotor }}[\mathrm{m}]$ & 1.530 \\
Hub-to-tip diameter ratio $\left[D_{\text {hub }} / D_{\text {rotor }}\right]$ & 0.29 \\
Rotor tip clearance $[\mathrm{m}]$ & 0.006 \\
Volumetric flow rate $\left[\mathrm{m}^{3} / \mathrm{s}\right]$ & 14.57 \\
Rotational speed $[\mathrm{rpm}]$ & 722 \\
\hline
\end{tabular}




\section{Appendix B}

\section{Periodic three-dimensional numerical model sensitivity analysis}

A sensitivity analysis is performed for the computational domain of the scale M-fan presented in Section 3.1. The next sections present the sensitivity analysis which initially determines mesh density and boundary displacement sensitivity with a zero tip clearance. This is to remove any influence the tip clearance may have on the solution. Thereafter, the tip clearance is introduced to determine the effect of mesh density within the tip region. As discussed in Section 3.4, all simulations are run with the same solution and scheme settings until the given convergence criteria is met.

\section{B.1 Mesh density sensitivity}

As previously discussed, the proposed meshing strategy produces a mesh of predominately structured hexahedral cells. cfMesh aligns the interior cells to a global coordinate system based on the bounding dimensions of the domain. This means that the vast majority of the cells are aligned to the flow direction which has been said to present a more accurate solution when compared to unstructured meshes (Louw, 2015).

The mesh consists of refinement regions around the blade which successively decrease in resolution further away from the blade until the maximum permissible cell sized is reached. This allows for a reduction in the overall cell count as high resolution refinement is only required in regions of particular interest.

In order to conduct a mesh density sensitivity analysis, initial inlet and outlet patch displacement lengths are required. Since the effects of boundary displace- 
ment are yet to be tested, the initial domain dimensions are chosen based on that used in literature.

During a numerical sensitivity analysis conducted on the M-fan, Wilkinson (2017) found that, for an annular duct configuration with a uniform inlet velocity profile and a fixed reference pressure outlet, a nondimensionalized inlet and outlet axial length of $l_{z} / D_{\text {rotor }}=0,547$ from leading and trailing edge, respectively, provided for a converged solution. Therefore, the initial inlet length for this sensitivity analysis is chosen to be equivalent to that used by Wilkinson (2017).

As the outlet pressure field is set to a zeroGradient boundary condition, the initial selection to use the same outlet domain length of Wilkinson (2017) would be superfluous. Instead an outlet axial length of a minimum of two chord fractions from trailing edge is chosen as done for the same outlet condition as Corsini and Sheard (2007a), Corsini et al. (2007b), Corsini et al. (2010) and Corsini and Sheard (2013).

Figure B.1 shows a partially sectioned view of the scale M-fan rotor region (RR) illustrating the various mesh refinements. As can be seen, the refinement regions are centralized around the blade and successively decrease in density as the distance from the blade is increased.

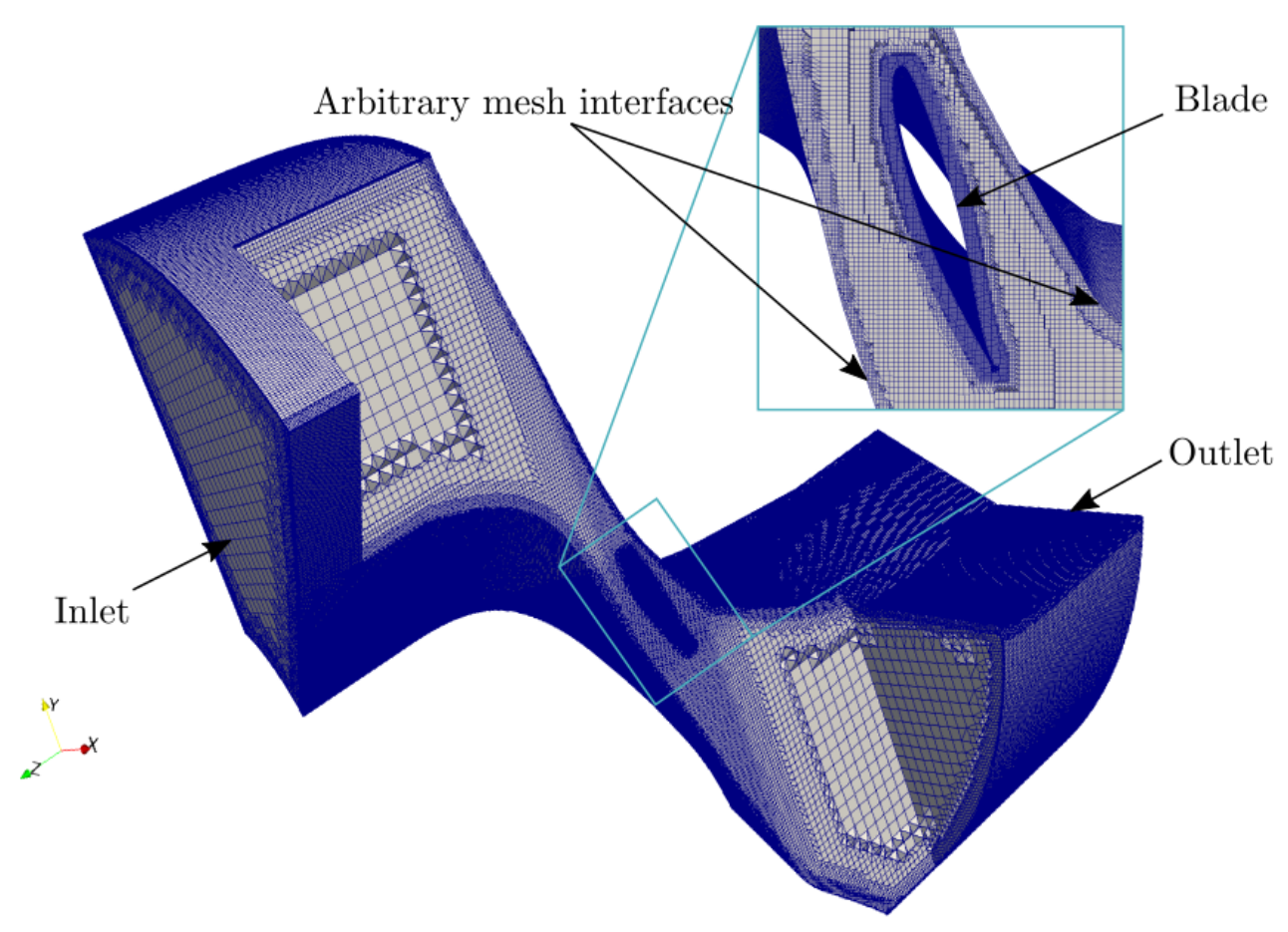

Figure B.1: Rotor region mesh refinement 


\section{APPENDIX B. PERIODIC THREE-DIMENSIONAL NUMERICAL MODEL} SENSITIVITY ANALYSIS

An initial mesh density (datum mesh) is chosen based on the minimum requirements for which the mesh quality parameters presented in Table 3.1 are met. Thereafter, the size and density of the refinement regions as well as the individual surface patch refinements are sequentially refined by a minimum factor of two in order to determine the effect. Table B.1 shows the results obtained from the mesh density sensitivity analysis at the calculated design flow rate of Wilkinson et al. (2019):

Table B.1: Mesh density sensitivity of the scale M-fan domain at the $14.68 \mathrm{~m}^{3} / \mathrm{s}$ calculated design flow rate of Wilkinson et al. (2019) for a $0 \mathrm{~mm}$ tip clearance

\begin{tabular}{ccccc}
\hline Mesh & Cell count & $\boldsymbol{\Delta}_{\boldsymbol{t}-\boldsymbol{s}}[\mathrm{Pa}]$ & $\boldsymbol{\eta}_{\boldsymbol{t}-\boldsymbol{s}}[-]$ & Comments \\
\hline 1 & $4.1\left(10^{6}\right)$ & 96.07 & 0.529 & Datum mesh \\
2 & $7.4\left(10^{6}\right)$ & 91.70 & 0.514 & Rotor region (RR) refinement \\
3 & $8.9\left(10^{6}\right)$ & 96.39 & 0.530 & Max cell size (MCS) refinement \\
4 & $10.8\left(10^{6}\right)$ & 91.28 & 0.513 & Mesh 2 + AMI surface refinement \\
5 & $13.3\left(10^{6}\right)$ & 90.77 & 0.513 & Mesh 4 + Mesh 3 \\
6 & $13.7\left(10^{6}\right)$ & 90.39 & 0.511 & Mesh 5 + increased RR refinement \\
\hline
\end{tabular}

Comparing mesh 3 to mesh 1 (datum mesh), an insignificant change in performance is apparent through the reduction of the maximum cell size (MCS). However, in comparing mesh 2 to the datum mesh, it can be seen that increasing the RR refinement has a significant effect on the change in fan performance (by up to a 4.55 percent change in total-to-static pressure rise). Therefore, this will be the area of focus in later refinements.

Mesh 4 compares the effect of AMI interpolation weights (which are responsible for carrying information between the cyclic patches) by refining these regions using mesh 2 as a baseline. The results are shown to change the solution by a maximum of 0.47 percent in total-to-static pressure rise when compared to mesh 2. Mesh 5 incorporates all the mesh refinements thus far mentioned. The fan total-to-static pressure rise is shown to change by a maximum of 0.55 percent when compared to mesh 4. Finally, mesh 6 increases the RR refinement of mesh 5 with the results showing a maximum change in total-to-static pressure rise of 0.42 percent.

From this it can be concluded that the RR refinement and AMI surface refinement are most influential to the solutions result. Comparing mesh 2 to mesh 4 through 6 , a negligible change in performance is apparent. Therefore, the refinement settings used to produce mesh 4 are used as the maximum settings for all future meshes since this provides for a conservative intermediate between the solutions. 
APPENDIX B. PERIODIC THREE-DIMENSIONAL NUMERICAL MODEL SENSITIVITY ANALYSIS

\section{B.2 Inlet/Outlet patch displacement sensitivity}

The effect of the inlet and outlet patch displacement on the solution needs to be determined in order to compensate for an unknown inlet velocity profile and an unknown effect of the far field wake downstream of the fan blade at the outlet.

Using the final mesh, as selected in the previous section, the inlet and outlet patch lengths are individually altered from blade leading and trailing edge, respectively, to see the effect on fan performance. Since the initial outlet patch displacement (being 2 chord fractions from trailing edge) has the greatest unknown with regards to a sensitivity analysis; this effect is tested first. Table B.2 gives the obtained results.

Table B.2: Outlet length sensitivity of the scale M-fan domain at the $14.68 \mathrm{~m}^{3} / \mathrm{s}$ calculated design flow rate of Wilkinson et al. (2019) for a $0 \mathrm{~mm}$ tip clearance

\begin{tabular}{cccc}
\hline Mesh & $\boldsymbol{l}_{\text {outlet }} / \boldsymbol{D}_{\text {rotor }}[-]$ & $\boldsymbol{\Delta} \boldsymbol{P}_{\boldsymbol{t}-\boldsymbol{s}}[\mathrm{Pa}]$ & $\boldsymbol{\eta}_{\boldsymbol{t}-\boldsymbol{s}}[-]$ \\
\hline 1 & 0.226 & 91.28 & 0.513 \\
2 & 0.401 & 90.61 & 0.509 \\
3 & 0.547 & 90.33 & 0.508 \\
\hline
\end{tabular}

The results indicate that the greatest change in fan performance lies between mesh 1 and mesh 2. Thereafter, a negligible change in performance is apparent with a further increase in outlet patch displacement. Therefore, the nondimensionalized axial outlet length of $l_{\text {outlet }} / D_{\text {rotor }}=0,401$ is chosen as a maximum for all future meshes.

Thereafter, the inlet patch displacement is tested to see the effect. Table B.3 shows the comparisons in performance of the various inlet patch displacement lengths.

Table B.3: Inlet length sensitivity of the scale M-fan domain at the $14.68 \mathrm{~m}^{3} / \mathrm{s}$ calculated design flow rate of Wilkinson et al. (2019) for a $0 \mathrm{~mm}$ tip clearance

\begin{tabular}{cccc}
\hline Mesh & $\boldsymbol{l}_{\text {inlet }} / \boldsymbol{D}_{\text {rotor }}[-]$ & $\boldsymbol{\Delta} \boldsymbol{P}_{\boldsymbol{t}-\boldsymbol{s}}[\mathrm{Pa}]$ & $\boldsymbol{\eta}_{\boldsymbol{t}-\boldsymbol{s}}[-]$ \\
\hline 1 & 0.273 & 93.57 & 0.522 \\
2 & 0.547 & 90.61 & 0.509 \\
3 & 0.820 & 88.56 & 0.502 \\
4 & 1.094 & 86.75 & 0.494 \\
\hline
\end{tabular}


APPENDIX B. PERIODIC THREE-DIMENSIONAL NUMERICAL MODEL SENSITIVITY ANALYSIS

A constant decrease in fan total-to-static pressure rise is apparent between all subsequent meshes despite increasing the inlet patch displacement. This could be due to increased losses as the inlet patch displacement length is increased. Therefore, in order to limit the losses incurred while also allowing the development of the inlet velocity profile, an axial inlet length of $l_{\text {inlet }} / D_{\text {rotor }}=0,820$ is chosen as this is a more conservative solution and provides for the best compromise.

\section{B.3 Boundary layer thickness effects within the tip region}

Due to the introduction of an unrealistic hub and casing length, when compared to that in practical application, it is possible that this could have a significant effect on the boundary layer development upstream of the fan blade (as previously discussed in Chapter 2.2.4). Therefore, in order to account for this, a slip boundary condition is applied as illustrated in Figure B.2.

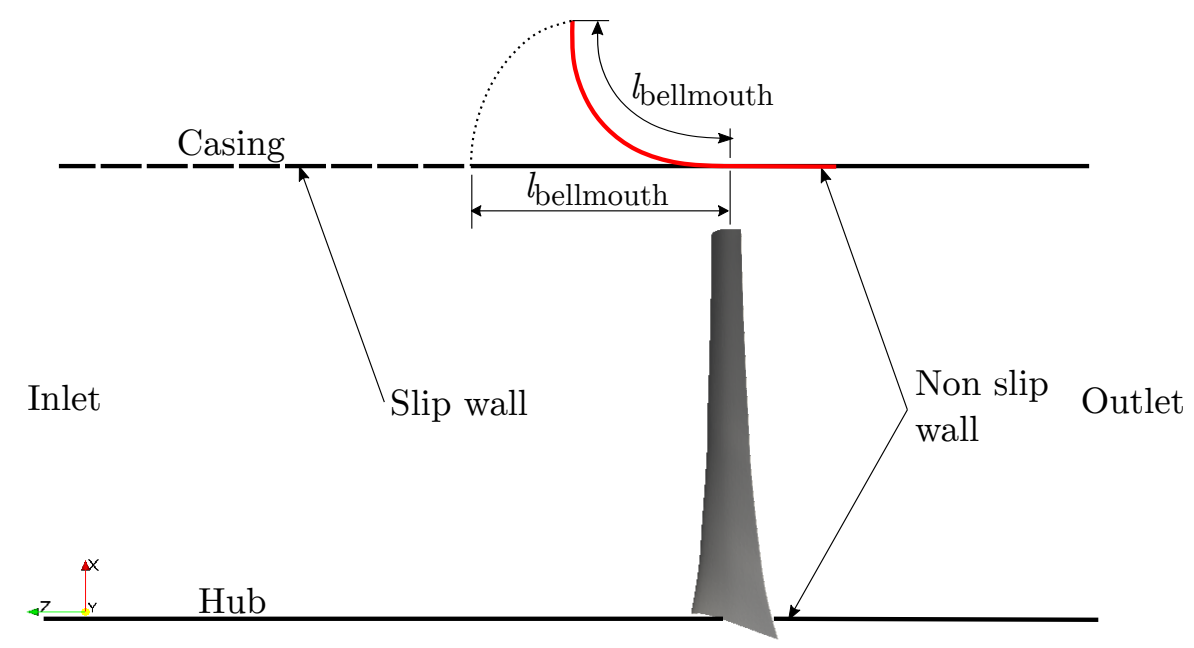

Figure B.2: Wall boundary allocation

As shown, the wall boundary on the casing is simply extended upstream of the blade to a length equal to that of the bellmouth; after which a slip boundary condition is applied up until the inlet. This gives a more representative boundary layer development up to the tip clearance region. It should be noted that in application the bellmouth causes an acceleration of the flow at the entrance of the tip region thereby reducing the boundary layer thickness, however, although 
APPENDIX B. PERIODIC THREE-DIMENSIONAL NUMERICAL MODEL SENSITIVITY ANALYSIS

the casing is completely flat in the set-up, the difference in the boundary layer thickness is assumed negligible.

\section{B.4 Tip clearance mesh density sensitivity}

Finally, the tip clearance is introduced to see the effect on the solution by varying the tip clearance mesh density. The largest tip clearance $(6 \mathrm{~mm})$ is chosen as this would incur the greatest losses and therefore have the greatest effect on fan performance. The bounding dimensions of the refinement region are chosen such that they surround the developed TLV near the tip region. A radial length from the fan's casing to 91.6 percent blade span and extending one chord length downstream from the blade's trailing edge is found to satisfy this requirement. Table B.4 gives the results obtained during the sensitivity analysis.

Table B.4: Tip clearance mesh density sensitivity of the scale M-fan domain at the $14.68 \mathrm{~m}^{3} / \mathrm{s}$ calculated design flow rate of Wilkinson et al. (2019) for a $6 \mathrm{~mm}$ tip clearance

\begin{tabular}{ccccc}
\hline Mesh & $\begin{array}{c}\text { No. spanwise } \\
\text { tip elements }\end{array}$ & Cell size $[\mathrm{m}]$ & $\boldsymbol{\Delta}_{\boldsymbol{t}-\boldsymbol{s}}[\mathrm{Pa}]$ & $\boldsymbol{\eta}_{\boldsymbol{t}-\boldsymbol{s}}[-]$ \\
\hline 1 & 27 & 0.008 & 74.70 & 0.459 \\
2 & 31 & 0.004 & 74.68 & 0.458 \\
\hline
\end{tabular}

Increasing the tip clearance mesh density produces an insignificant improvement in fan performance. This suggests that the tip clearance mesh density of mesh 1 is sufficient to be used for the remainder of the simulations. A reduction in tip clearance would mean that the spanwise cell count within the tip gap would be reduced. Therefore, the spanwise cell count of mesh 1 is taken as a minimum for all future meshes. 


\section{Appendix C}

\section{Turbulence properties}

In order to model the turbulent flow around the blade, a set of initial turbulence parameters need to be estimated. This is in order to impose the turbulent values over the inlet patch to model a more realistic inlet flow condition as that of the experimental set-up. The process of determination of such parameters is herewith discussed.

\section{C.1 Estimation of turbulent kinetic energy from turbulence intensity}

The turbulent kinetic energy is related to the mean free stream velocity and turbulence intensity by the following equation:

$$
k=\frac{3}{2}\left(U_{\infty} I\right)^{2}
$$

Since the inlet patch is placed far away from the blade, the turbulence levels should not have a significant effect on the final solutions result. Nevertheless, a value of 1 percent for turbulence intensity, as determined to be the case at Stellenbosch University's fan test facility (Louw, 2015), is used in this study.

\section{C.2 Estimation of turbulent dissipation/specific dissipation rate from mixing length scale}

Since the current case makes use of an annular set-up, the most similar case is that of the pipeCyclic tutorial in OF. In this tutorial, the mixing length is determined using 


$$
l_{\text {mix }}=0.5\left(l_{\text {span }}\right)
$$

where $l_{\text {span }}=\frac{D_{\text {rotor }}-D_{\text {hub }}}{2}$ which represents the hydraulic diameter of the annulus. This formula assumes the largest mixing length for a fully developed pipe flow to be that of 50 percent of the channel height. Using this formula along with the turbulent kinetic energy, the turbulent dissipation rate and turbulent specific dissipation rate are determined by the following equations:

$$
\begin{aligned}
& \epsilon=C_{\mu}^{3 / 4} \frac{k^{3 / 2}}{l_{\text {mix }}} \\
& \omega=\frac{k^{1 / 2}}{C_{\mu}^{1 / 4} l_{\text {mix }}}
\end{aligned}
$$

where $C_{\mu}$ represents the empirical turbulence model constant, typically given as 0.09 . 


\section{Appendix D}

\section{Post-processing}

The procedures used to quantify the flow near the blade tip is discussed. Postprocessing is conducted using the OF post-processing tool ParaView v5.4.0.

\section{D.1 Tip leakage flow quantification}

Figure D.1 illustrates the procedure used to determine the area-weighted average relative TLF velocity, $W_{T L F}$, within the tip gap at each chord-wise interval for the $6 \mathrm{~mm}$ datum fan case.

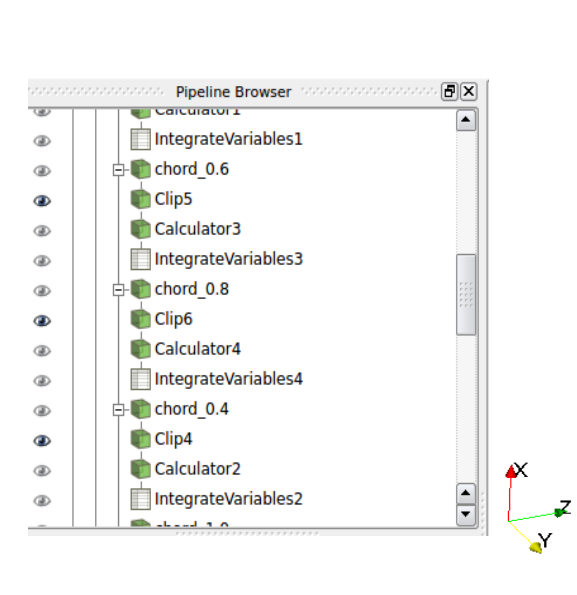

a)

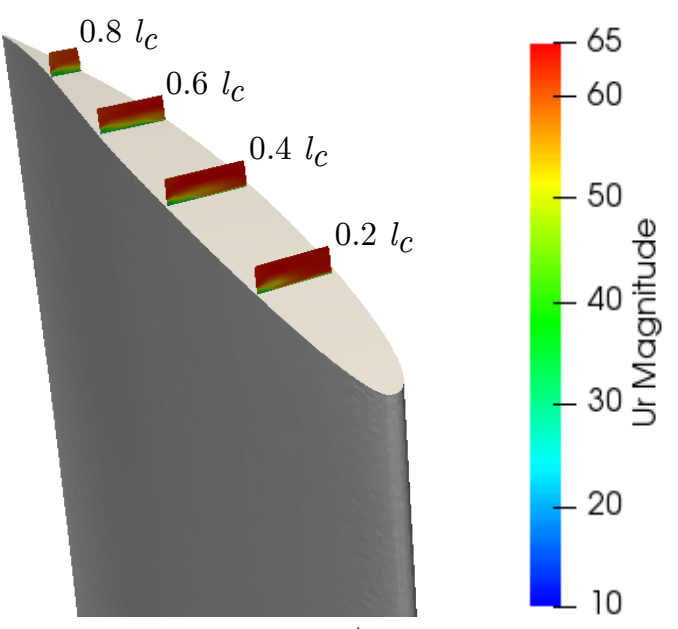

b)

Figure D.1: Area-weighted average relative tip leakage flow velocity postprocessing: a) Paraview filter example and b) Paraview visualization 
As shown in Figure D.1 a, a slice filter is used to evaluate the TLF at 20 percent intervals along the blade chord. The clip filter is then used to localize each slice area to be within the tip gap. The final clipped regions are illustrated in Figure D.1b at each chord-wise location. Finally, the integrate filter is used over each chord-wise interval to determine the area weighted average relative TLF velocity.

\section{D.2 Tip leakage vortex quantification}

Figure D.2 illustrates the procedure used to determine the TLV exit trajectory angle, $\beta_{T L V}$, for the $6 \mathrm{~mm}$ datum fan case.

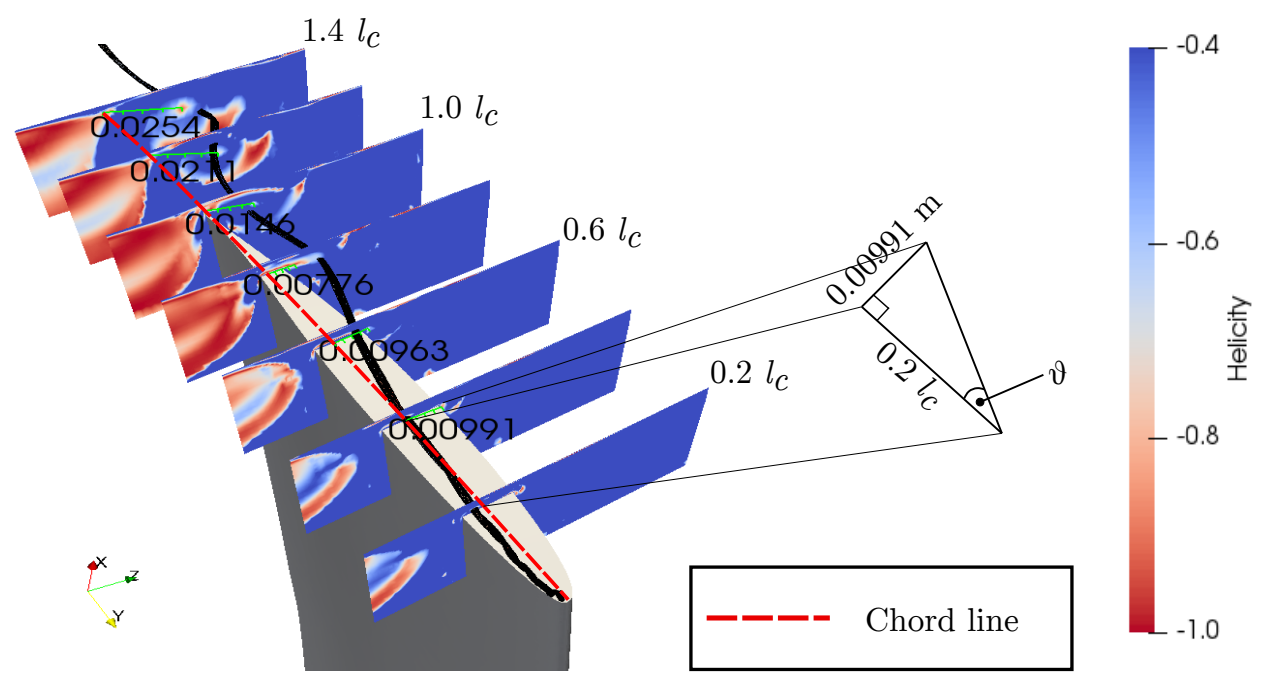

Figure D.2: Tip leakage vortex exit trajectory angle post-processing

As shown, the helicity contour plot scaling range is reduced in order to increase the contrast of the vortex core. This is in order to be able to accurately determine the numerical location of the vortex core. From this, Paraview's ruler function is used to measure the perpendicular distance between the vortex core and the blade chord at 20 percent chord intervals. This is done to prevent any error magnification of the $\beta_{T L V}$ angle. After this, basic trigonometry is used to determine the angle, $\theta$, between each chord-wise abscissae and the vortex core. An example is shown in the figure. 


\section{Appendix E \\ Design method}

The following chapter details the method used in the construction of the TE endplate design. The required apparatus is listed as follows:

- 8 carbon fibre M-fan blades

- $150 \mathrm{~g} / \mathrm{m}^{2}$ biaxial $\left(+/-45^{\circ}\right)$ carbon fabric $(880 \times 30.5 \mathrm{~mm})$

- SR 1280 / SD 4775 epoxy resin

- Foam roller

- Digital scale $2 \mathrm{~kg} \times 0.1 \mathrm{~g}$

- Non-latex nitrile gloves

- Paper plate and cup

- Wooden depressor stick

- Plywood sheets

- 2 mylar plastic sheets $(200 \times 200 \mathrm{~mm}$ each $)$

- Scissors

- Utility knife

- Fan heater

- Masking tape and black pen

- A small grinder 
- Vernier caliper

- 800 grit sandpaper and sanding block

- High density foam sheets

- Weights

A layer-up approach is used to create the basic design shape as this method minimizes the material introduced near the blade tip while still allowing for a smooth surface transition. Using the 2-dimensional aerofoil profile near the blade tip, two stencils (each comprising of a bottom half, aerofoil, and top half cross section) are cut from plywood sheets at the same incidence angle as that of the blade tip and $110 \mathrm{~mm}$ radially from the blade tip. This is done in order to capture the blade's washout to ensure the blades seat correctly in the mould. Both stencils extend outward from the blade's trailing edge at the desired end-plate setting angle and at a slightly increased end-plate length. The stencils are placed on either side of a high density foam block which is then cut using a hot wire. An illustration of the approach and initial set-up is given in Figure E.1 a and b respectively. 


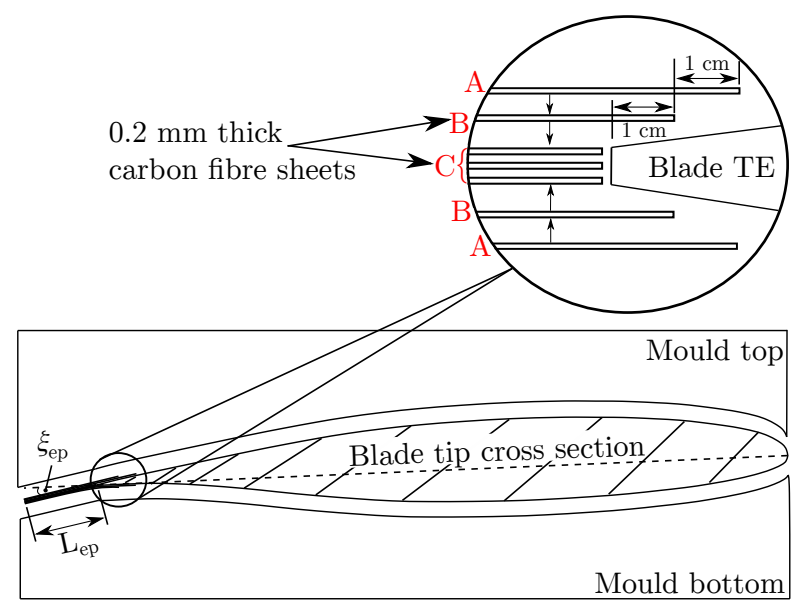

(a) Layer-up approach

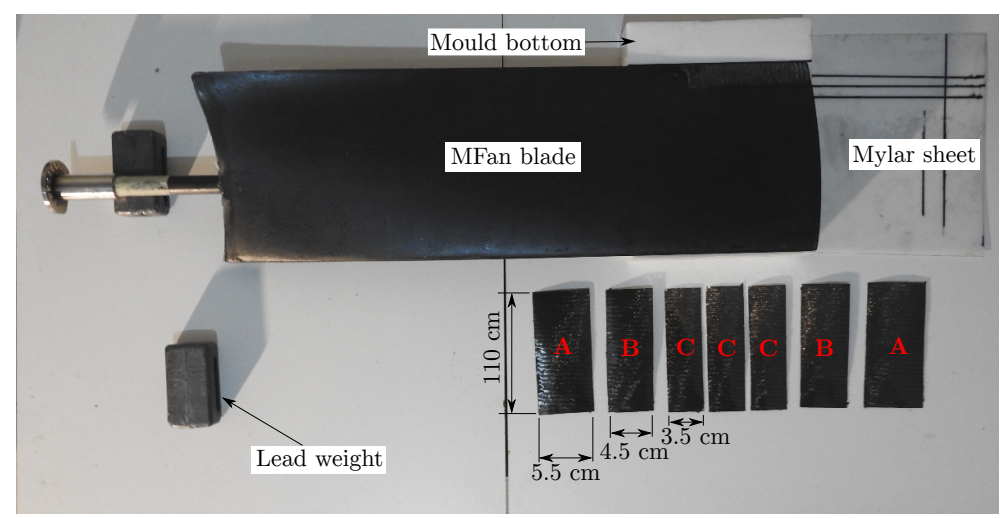

(b) Set-up

Figure E.1: Design construction approach and initial set-up

As illustrated, the design is composed of 7 layers of $0.2 \mathrm{~mm}$ thick carbon fibre sheets arranged such that two of the sheets overlap the blade's TE on either side by at least $1 \mathrm{~cm}$ each. This is done in order to ensure a smooth transition between surfaces while additionally providing a secure attachment for the design. Three central layers are chosen to allow the design core to have the same thickness as that of the blade's TE (further aiding in the smooth transition between surfaces).

Using a digital scale and paper cup, the correct weight ratios of resin to hardener are first measured and then mixed using a wooden depressor stick. To ensure a thorougher mixing of the solution, the end of the depressor stick is squared (to be able to scrape the solution from the corners of the cup) and mixing is done for a minimum of 5 mins. A total solution weight of $44.5 \mathrm{~g}$ was found to be sufficient for 
two designs (note: this value takes the loss through absorption of the roller into consideration). Non-latex gloves are used when handling the chemicals to prevent contact and subsequent contamination.

Once thoroughly mixed, the epoxy solution is poured onto a paper plate and a roller is then used to spread the solution over the carbon fibre sheets. Once well saturated, the carbon fibre/epoxy sheets are individually layered (initially in the order ABCCC according to Figure E.1p onto a mylar sheet and aligned on top of the mould bottom. It should be noted that both sides of the carbon fibre sheets are well saturated to ensure that the epoxy resin properly impregnates the fibres. An example of the process is shown in Figure E.2a.

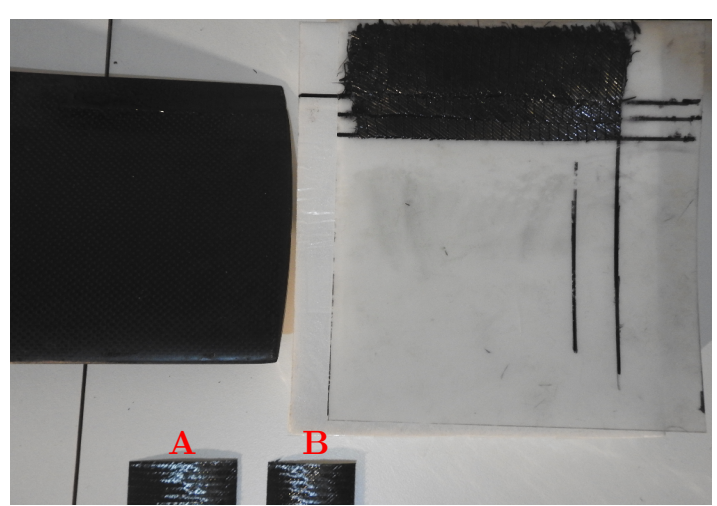

a) Initial

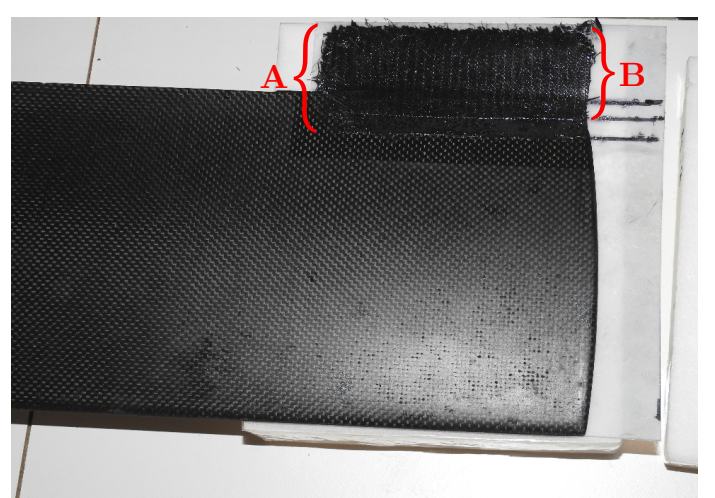

b) Final

Figure E.2: Layering method

The blade is then placed on top of the mould so that the final layers may be added in a similar fashion as done previously (in the order BA as illustrated in Figure E.2b) to complete the layering process. Once complete, a final mylar sheet and the mould top half are added to interpose the carbon/epoxy sheets. Weights are then added on top of the mould (with bias given towards the blade's TE) to ensure sufficient pressure when curing. A fan heater is then turned on and placed near the modified blade where the entire set-up is enclosed under a box to ensure good insulation (the box should preferably be made from high density foam but cardboard was found to be sufficient for the current application). The final set-up before curing is given in Figure E.3. 


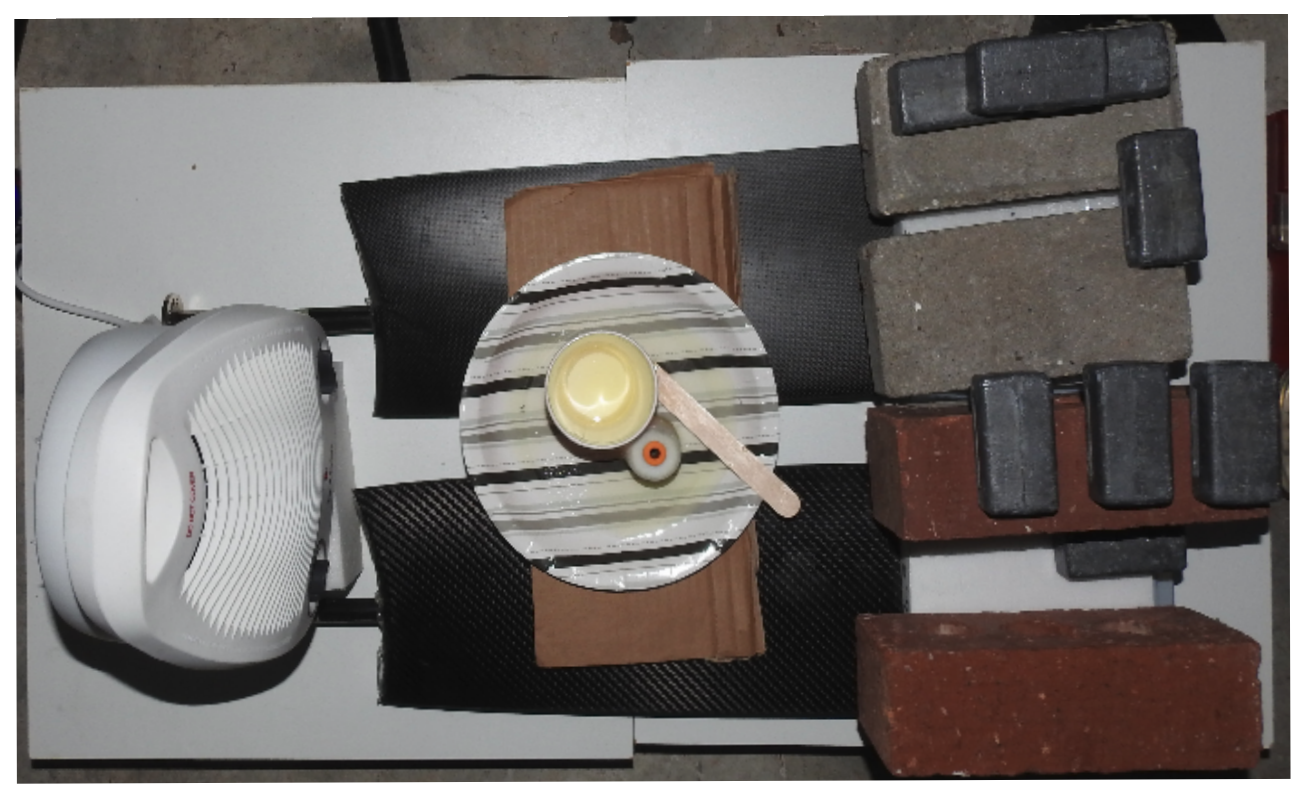

Figure E.3: Pre-curing set-up

Curing normally takes between 6 and 10 hours (depending on the ambient temperature). The mixing cup and paper plate are intentionally included to make certain that the epoxy has cured. Once cured, the blades are removed from the moulds and marked to the appropriate dimensions using masking tape and a vernier caliper (as illustrated in Figure E.4a).

A small grinder is then used to cut the end-plate to the basic shape; after which a sanding block is used to refine the dimensions to an acceptable tolerance $( \pm 0.1 \mathrm{~mm})$ using the blade's TE as a reference datum. As a bit of overflow of the epoxy resin is expected during curing, the grinder is again used to sand the intersection between the blade and end-plate surface to ensure a smooth transition. Finally, a clear lacquer is sprayed over the design to fill any imperfections. The final end-plate construction is illustrated in Figure E.4b. 


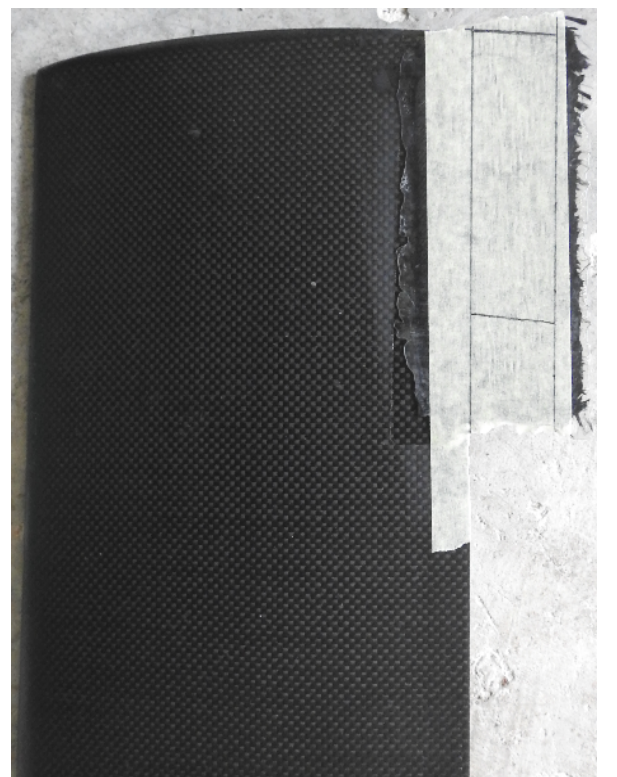

a) Before cutting

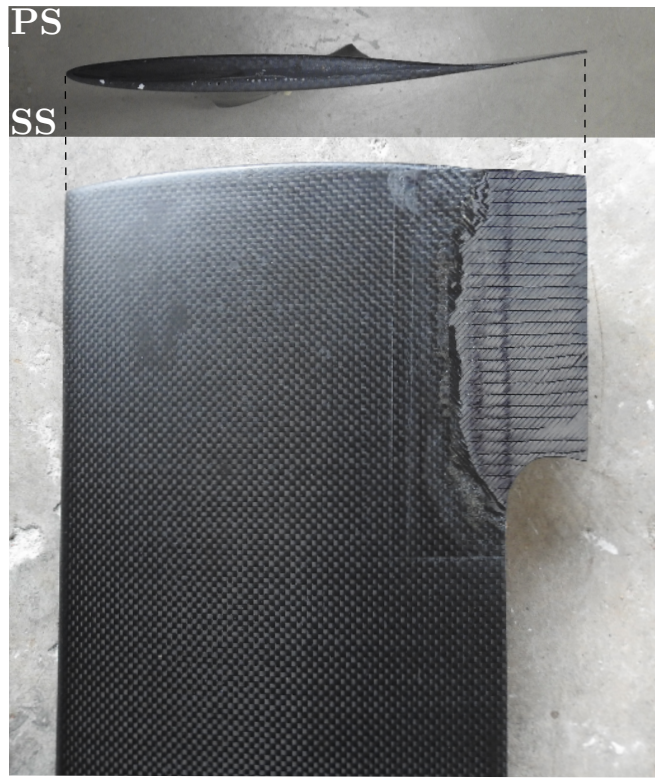

b) Final design

Figure E.4: Final end-plate design 


\section{Appendix F}

\section{Structural feasibility analysis}

A structural feasibility analysis is required in order to asses the minimum required thickness needed for practical implementation. As such, a static analysis is conducted using statically determinate beam theory to simplify the problem. The worst case scenario is chosen for analysis which would correlate to the design with the greatest overall stress; that being the optimized end-plate design with the greatest end-plate setting angle at the lowest evaluated flow rate of $9.29 \mathrm{~m}^{3} / \mathrm{s}$.

\section{F.1 Displacement analysis}

For convenience, the dimension labelling convention for the TE end-plate design is included in Figure F.1.

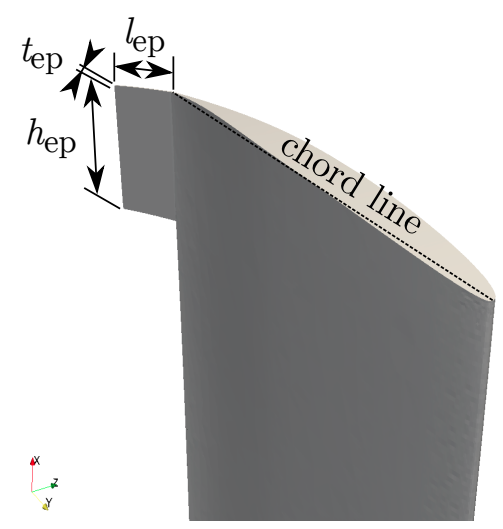

Figure F.1: Trailing edge end-plate dimensions

As the predominant forces exerted on the TE end-plate design would be due to the fluid flow interaction, only these forces will be considered. Therefore, the numerical 
forces acting on the end-plate design (as determined from the results obtained in Chapter 9p are given in Table F.1.

Table F.1: Numerical forces acting on the optimized TE end-plate design evaluated at $\dot{V}=9.29 \mathrm{~m}^{3} / \mathrm{s}$

\begin{tabular}{cccc}
\hline & \multicolumn{3}{c}{ Cartesian direction } \\
& $\mathbf{x}$ & $\mathbf{y}$ & $\mathbf{z}$ \\
\hline Force $[\mathrm{N}]$ & -2.2018 & -1.1600 & 6.7788 \\
\hline
\end{tabular}

Due to the purpose of this investigation being purely to confirm design feasibility, simplified cantilever beam theory is used to represent the end-plate's displacement during operation. Therefore, projecting these forces onto the end-plate's localized coordinate system, the overall force exerted on the end-plate is found to act perpendicular to the pressure side of the end-plate, with $F_{\text {total }}=8,8626 \mathrm{~N}$. This force can then be represented as a point load acting on the end-plate, located at $x=a$ from the blade's trailing edge. It should be noted that this force is an estimation of the true force and its location is simplified. Figure F.2 illustrates the fixed-free cantilever beam configuration which best describes the TE end-plate's set-up.

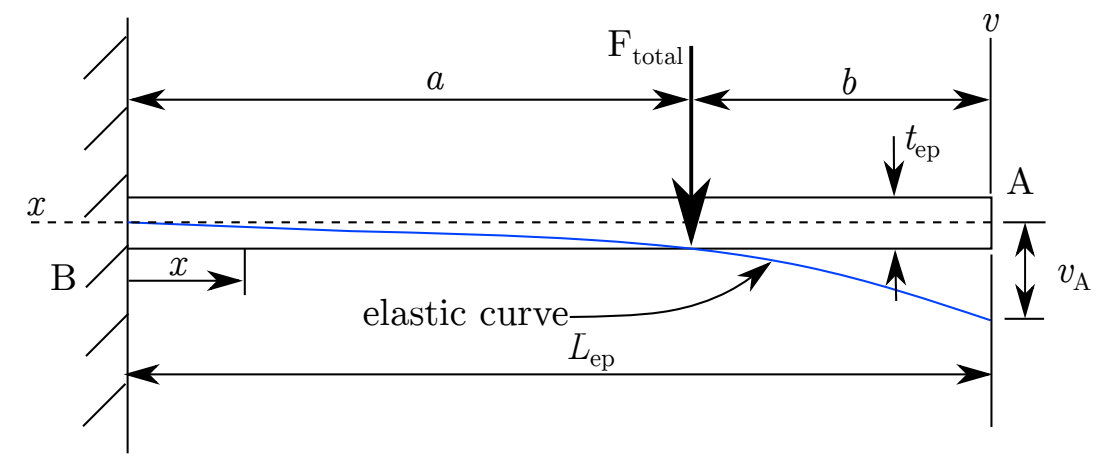

Figure F.2: Fixed-free cantilever beam set-up

Using the approximated elastic curvature equation to represent the beam's displacement,

$$
E I \frac{d^{2} v}{d x^{2}}=-F_{\text {total }} x
$$

and substituting the relevant boundary conditions $(d v / d x=0$ at $x=0$ and $v=0$ at $x=0$ ); the equation representing the beam's displacement as a function of $x$ is found through integration as 


$$
v(x)=-\frac{F_{\text {total }} a^{2}}{6 E I}(3 x-a) \quad \text { for } a<x<l_{e p}
$$

where $E$ represents the material's modulus of elasticity and $I$ represents the beam's moment of inertia about the neutral axis. Representing Equation F.2 in terms of known variables, the final equation used to represent the end-plate's maximum displacement is represented by

$$
v_{\text {max }}=-\frac{2 F_{\text {total }} a^{2}}{E h_{e p} t_{e p}^{3}}\left(3 l_{e p}-a\right)
$$

In order to determine the location $a$ where the force $F_{\text {total }}$ is acting, the static pressure coefficient $C p$ distribution midspan across the end-plate design is referred to in Figure F.3.

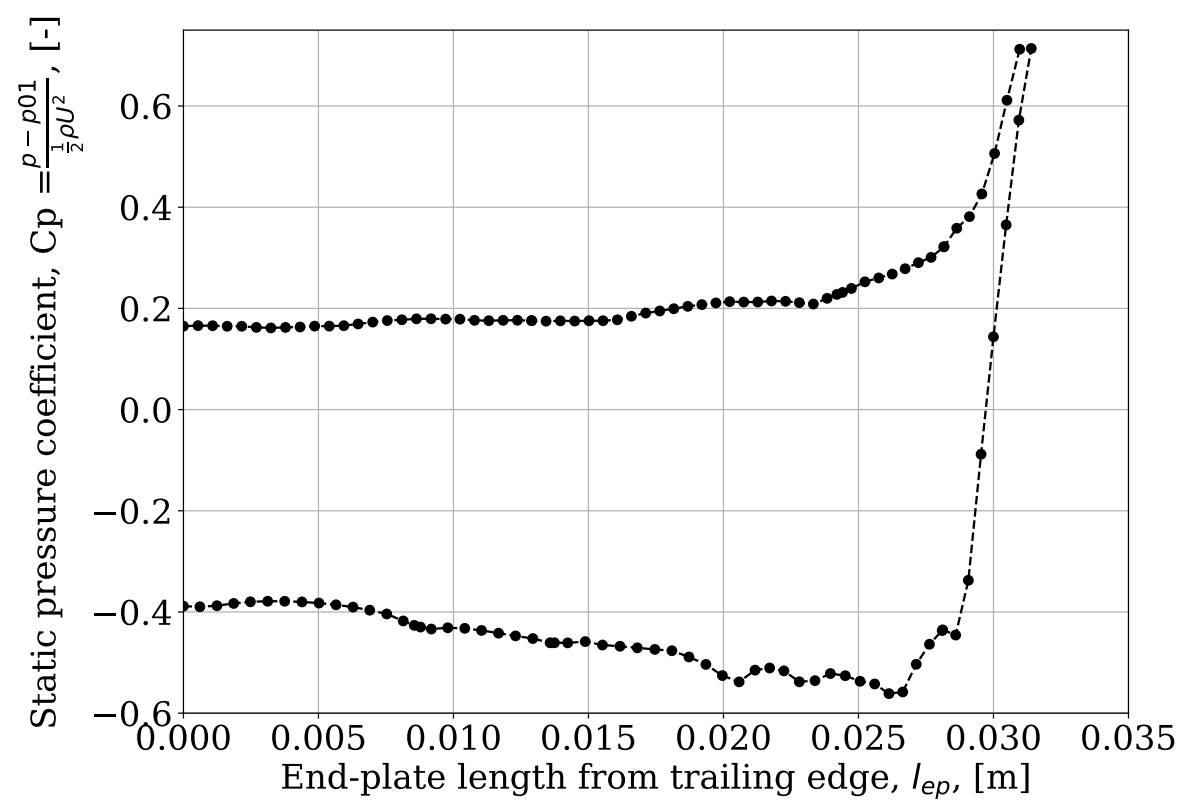

Figure F.3: Midpsan static pressure coefficient $C p$ distribution of the optimized TE end-plate design evaluated at $\dot{V}=9.29 \mathrm{~m}^{3} / \mathrm{s}$

From this, the location of the centre of pressure (COP) (which correlates to the location where $F_{\text {total }}$ acts) can be determined by calculating the centroid of the area enclosed within the $C p$ distribution curve. The equation representing the centroid of a complex shape is given by

$$
\bar{x}=\frac{\int_{A} x d A}{\int_{A} d A}=\frac{\sum_{i} \bar{x}_{i} \bar{A}_{i}}{\sum_{i} \bar{A}_{i}}
$$


Using Equation F.4, the centroid (and subsequently the COP) is found to be $\bar{x}=\mathrm{COP}=0,01580 \mathrm{~m}$ from the blade's trailing edge. Table F.2 gives the material properties of biaxial $\left(+/-45^{\circ}\right)$ carbon fabric/epoxy composite. This material is chosen for its high strength-to-weight ratio and torsional resistance (as discussed in Section 10.1.

Table F.2: Material properties of biaxial $\left(+/-45^{\circ}\right)$ carbon fabric/epoxy composite

\begin{tabular}{ccc}
\hline Property & Variable & Value \\
\hline Density $\left[\mathrm{m}^{3} / \mathrm{kg}\right]$ & $\rho$ & 1600 \\
Modulus of elasticity [GPa] & $E$ & 19.1 \\
Poissons ratio [-] & $\mathrm{v}$ & 0.74 \\
Tensile yield strength [MPa] & $\sigma_{Y}$ & 110 \\
\hline
\end{tabular}

Finally, Figure F.4 illustrates the maximum TE end-plate displacement $v_{\max }$ as a function of end-plate thickness $t_{e p}$.

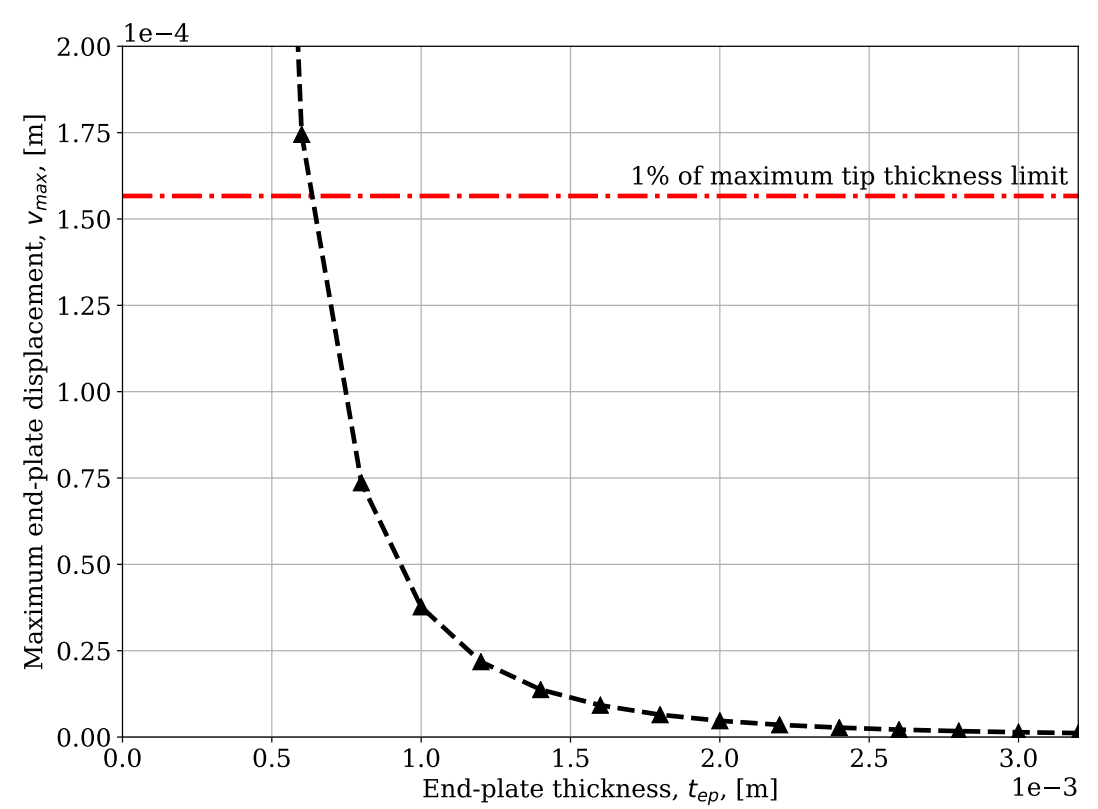

Figure F.4: Optimized TE end-plate maximum displacement vs end-plate thickness evaluated at $\dot{V}=9.29 \mathrm{~m}^{3} / \mathrm{s}$

As evident, the predicted displacement of the TE end-plate design falls below the maximum limit (set to be $1 \%$ of the blade's maximum tip thickness) at a total end-plate thickness of around $0.6 \mathrm{~mm}$ or greater. This limit is chosen as it provides 
for a conservative compromise between minimizing the end-plate's thickness and reducing its displacement. As the displacement for a $1.4 \mathrm{~mm}$ thick end-plate (alike to that of the implemented design as discussed in Section 10.1) is well below this limit, the current design's displacement is deemed acceptable for practical implementation.

\section{F.2 Stress analysis}

An annular disk is used to represent the stresses experienced within the end-plate. The stress distributions for the hoop stress, $\sigma_{\theta}$, and radial stress, $\sigma_{r}$, are shown in Figure F.5.

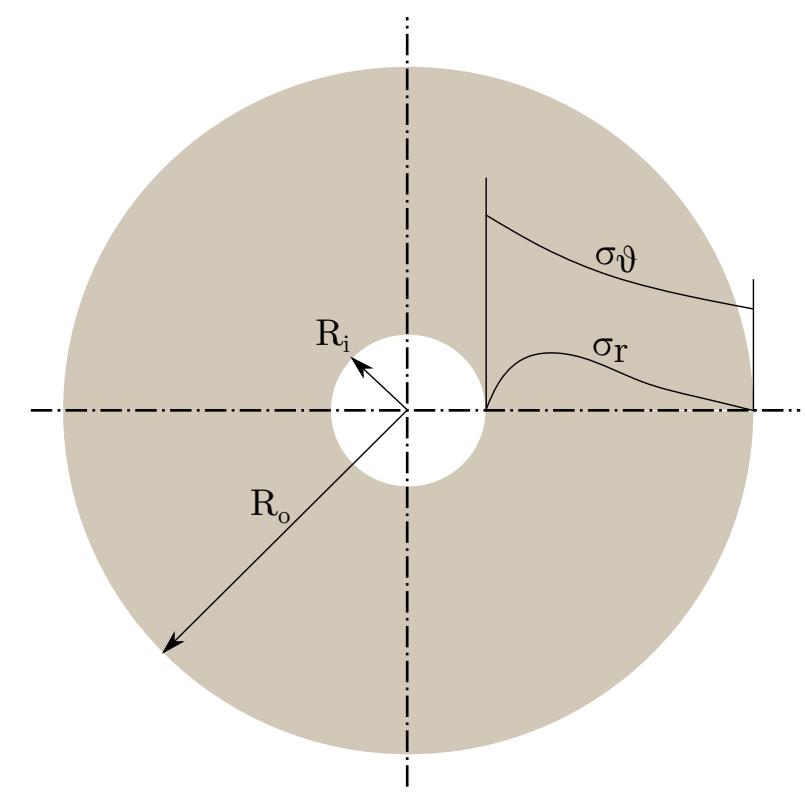

Figure F.5: Annular disk radial and hoop stress distributions

The size of the disk is representative of the actual end-plate design, with $R_{i}=$ $0.668 \mathrm{~m}$ and $R_{o}=0.765 \mathrm{~m}$. The maximum value of $\sigma_{\theta}$, located at $r=R_{i}$, is given by

$$
\left(\sigma_{\theta}\right)_{\max }=\frac{3+\mathrm{v}}{4} \rho \omega^{2}\left(R_{o}^{2}+\frac{1-\mathrm{v}}{3+\mathrm{v}} R_{i}^{2}\right)
$$

where $\omega=75,6 \mathrm{rad} / \mathrm{s}$ represents the angular velocity of the rotor. $\sigma_{r}$ is a maximum when $\frac{d \sigma_{r}}{d r}=0$ or $r=\sqrt{R_{i} R_{o}}$ (Benham et al., 1996); therefore the equation representing the maximum radial stress is given by 


$$
\left(\sigma_{r}\right)_{\max }=\frac{3+\mathrm{v}}{8} \rho \omega^{2}\left(R_{o}-R_{i}\right)^{2}
$$

with the axial stress contribution due to the fluid flow interaction being represented by

$$
\sigma_{a}=\frac{F_{t o t a l}}{A_{e p}}
$$

where $A_{e p}=l_{e p} \times h_{e p}$ is the area of the end-plate exposed to the flow. The von Mises principle stress equation used to represent the total stress on the end-plate is described by

$$
\sigma_{v}=\sqrt{\frac{1}{2}\left[\left(\sigma_{\theta}-\sigma_{r}\right)^{2}+\left(\sigma_{r}-\sigma_{a}\right)^{2}+\left(\sigma_{a}-\sigma_{\theta}\right)^{2}\right]}
$$

Substituting the relevant values into Equation F.8, the von Mises principle stress can be calculated. Table F.3 compares the material yield stress to the von Mises yield stress for the optimized TE end-plate design.

Table F.3: Optimized TE end-plate von Mises stress and carbon fabric/epoxy yielding stress comparison

\begin{tabular}{cc}
\hline Stress & Value $[\mathrm{MPa}]$ \\
\hline$\sigma_{v}$ & 5.25 \\
$\sigma_{Y}($ carbon $/$ epoxy $)$ & 19.10 \\
\hline
\end{tabular}

As evident, the von Mises yield stress criterion for the optimized end-plate design is sufficiently below the material's yield stress. This further suggests design feasibility with regards to material yielding when using the aforementioned carbon/epoxy composite material in the construction of the TE end-plate design. 


\section{Appendix G}

\section{Test facility calibration}

Each of the four measurement instruments at the test facility are individually calibrated prior to testing. The following sections further discuss details into the instrument calibration.

\section{G.1 Pressure transducer calibration}

Each of the two EH Deltabar PMD75 pressure transducers are calibrated using a Betz manometer. As illustrated in Figure G.1, a set of valves are used to isolate the transducers so that they are only connected to the manometer and an artificial pressure source.

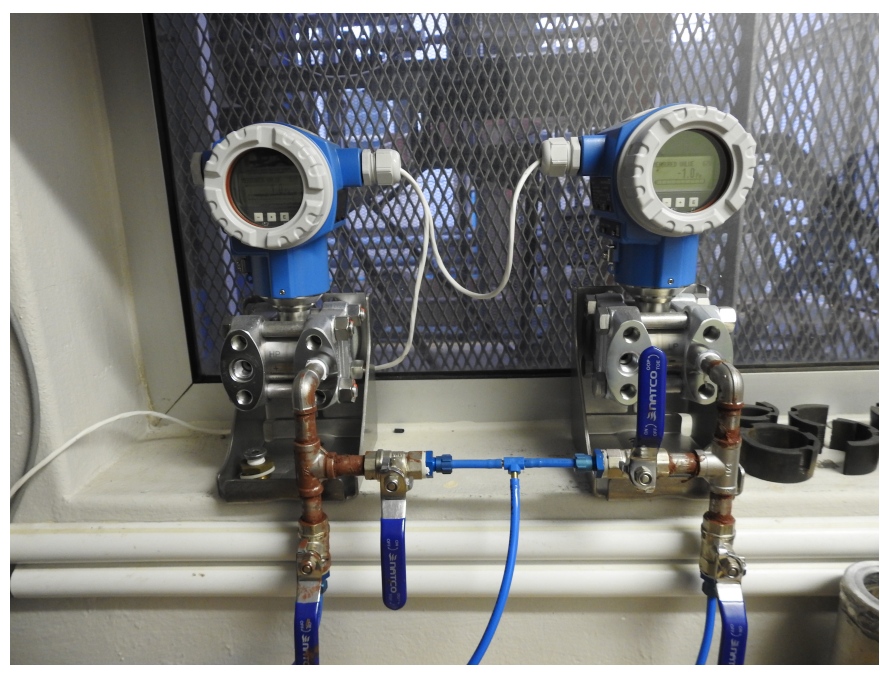

Figure G.1: Bellmouth (left) and plenum chamber (right) pressure transducers 
Once connected, the pressure source is then used to set a predefined pressure according to the manometer reading. The subsequent pressure reading and voltage output of the manometer and transducer are captured using the CATMAN software package. This process is repeated for each transducer using six data points (including that of the zero pressure reading). The following figure illustrates an example of the calibration results.

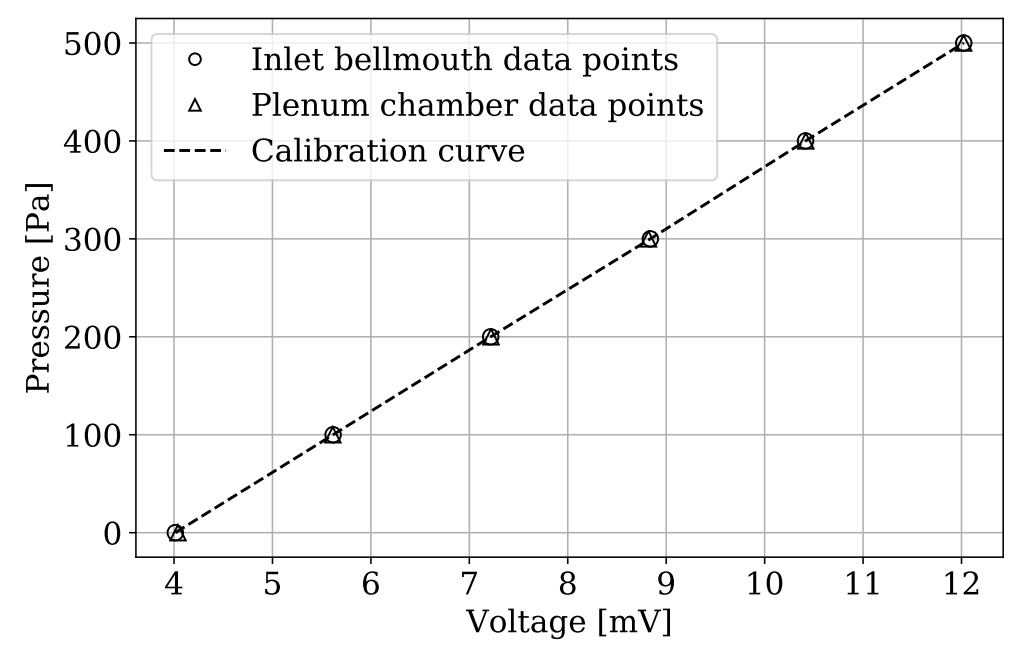

Figure G.2: Pressure transducer calibration results

Using these sets of discretized data points, a $2^{\text {nd }}$ order polynomial curve is fitted which is then used to interpolate between data points during testing. An example of such a curve for each pressure transducer is given in Table G.1.

Table G.1: Pressure transducer calibration trend data

\begin{tabular}{lcc}
\hline Transducer & Equation & $\mathbf{R}^{\mathbf{2}}$ \\
\hline Bellmouth EH Deltabar PMD75 & $p(V)=0,0320 V^{2}+61,9101 V-248,8605$ & 0.9999 \\
Plenum EH Deltabar PMD75 & $p(V)=-0,0511 V^{2}+63,4085 V-254,9948$ & 0.9999 \\
\hline
\end{tabular}

\section{G.2 Torque transducer calibration}

As illustrated in Figure G.3a, an adjustable weight system is used to introduce an artificial moment on the shaft in order to calibrate the HBM T22 torque transducer. With the system attached to the freely moving shaft, an adjustable weight (located to the left of the figure on the moment arm) is then used to balance the system in 
order to capture the shaft's zero torque reading. Thereafter, the shaft is anchored in place using a set of chains and tensioner hooks as illustrated in Figure G.3b.

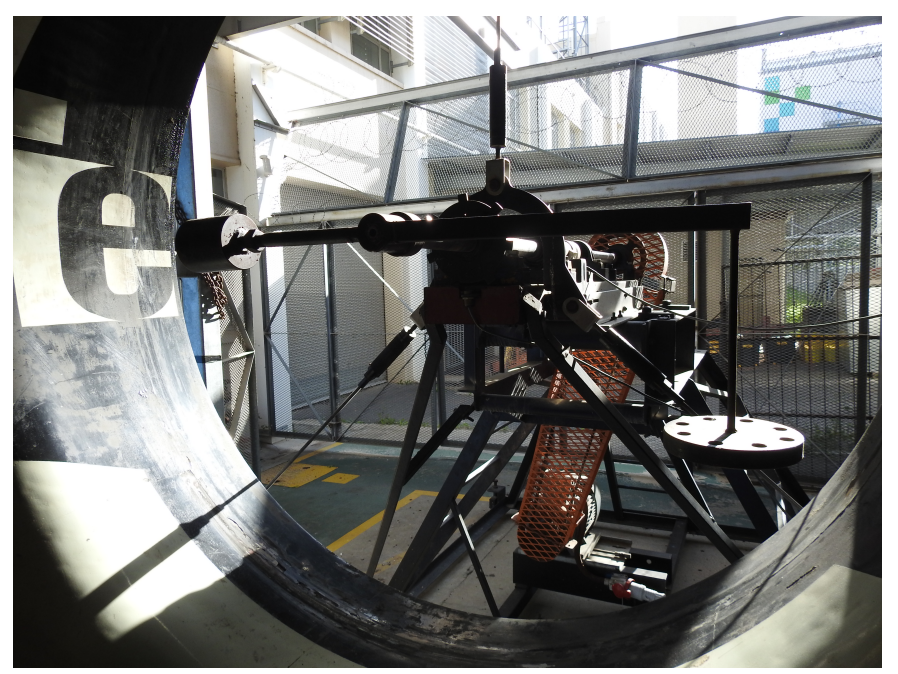

(a) Moment adjustment set-up

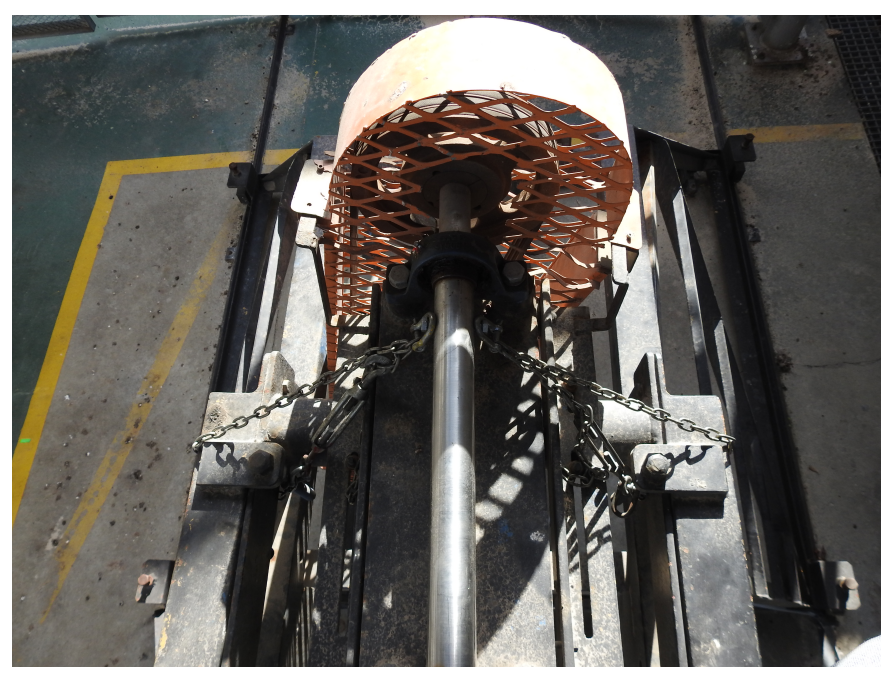

(b) Shaft anchoring

Figure G.3: Torque transducer calibration

Once sufficiently anchored, a set of four different weights are used to systematically increase the load at the end of the moment arm (located to the right of Figure G.3a). The CATMAN software package is again used to capture each of the torque readings. As the moment arm of the system is known, the resulting torque can be calculated and entered for each reading. An example of the torque calibration results is illustrated in Figure G.4. 


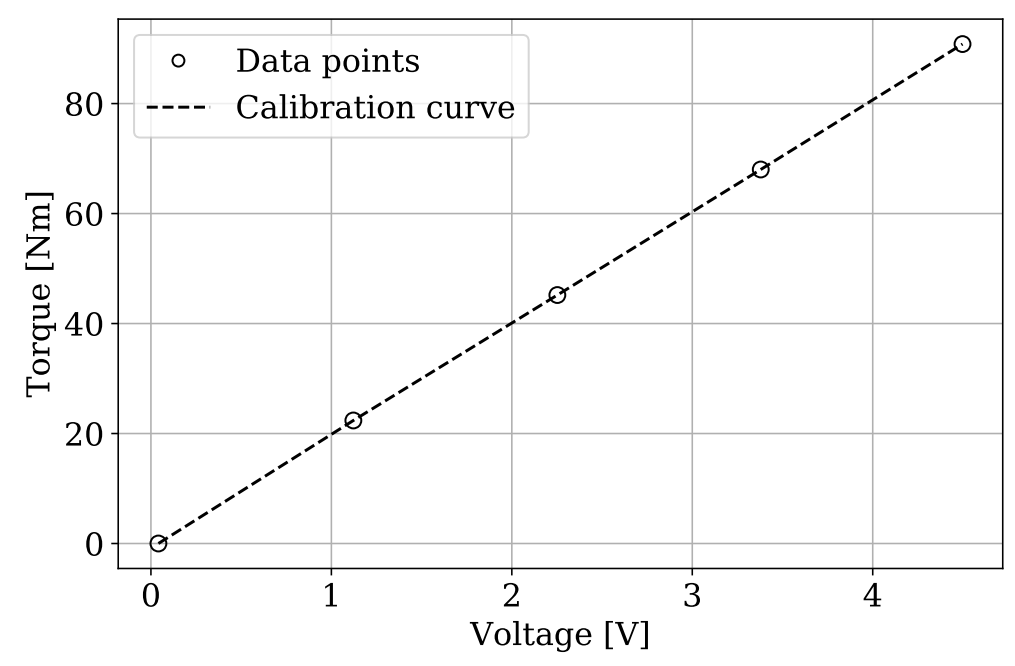

Figure G.4: Torque transducer calibration results

Similar to the pressure transducer calibration, the program fits a $2^{\text {nd }}$ order polynomial curve to the data which is then used for interpolation during testing. An example of the torque's transducer calibration data is given in Table G.2.

Table G.2: Torque transducer calibration trend data

\begin{tabular}{lcc}
\hline Transducer & Equation & $\mathbf{R}^{2}$ \\
\hline HBM T22 & $T(V)=-0,0328 V^{2}+20,4956 V-0,7637$ & 0.9999 \\
\hline
\end{tabular}

\section{G.3 Fan rotational speed calibration}

Finally, calibration of the proximity sensor (illustrated in Figure G.5) is performed through the use of a stroboscope. Using a piece of reflective tap that is attached to the end of the shaft, the stroboscope is used to determine the point at which the piece of reflective tap remains "stationery" on the shaft at a specified frequency input to the variable speed drive (VSD). Likewise with the other calibrations, the data point is then captured using the CATMAN software package with the rotational speed value being manually entered. This process is repeated for six data points with bias being given to values around the design point speed. 


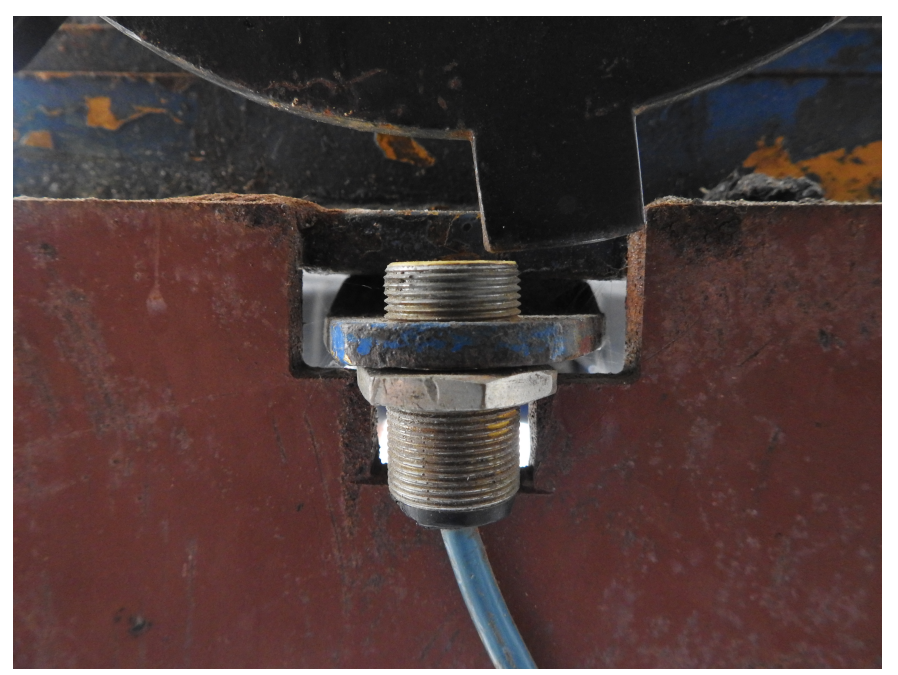

Figure G.5: Proximity sensor

It should be noted that this method is inherently subjective towards the accuracy of the operator's eye when taking the stroboscope measurements. However, despite this, the results obtained still provided sufficient relative accuracy (deviations of less than $0.01 \%$ ). Therefore, this method is deemed sufficient for use in the current application. An example of the rotational speed calibration is illustrated in Figure G.6.

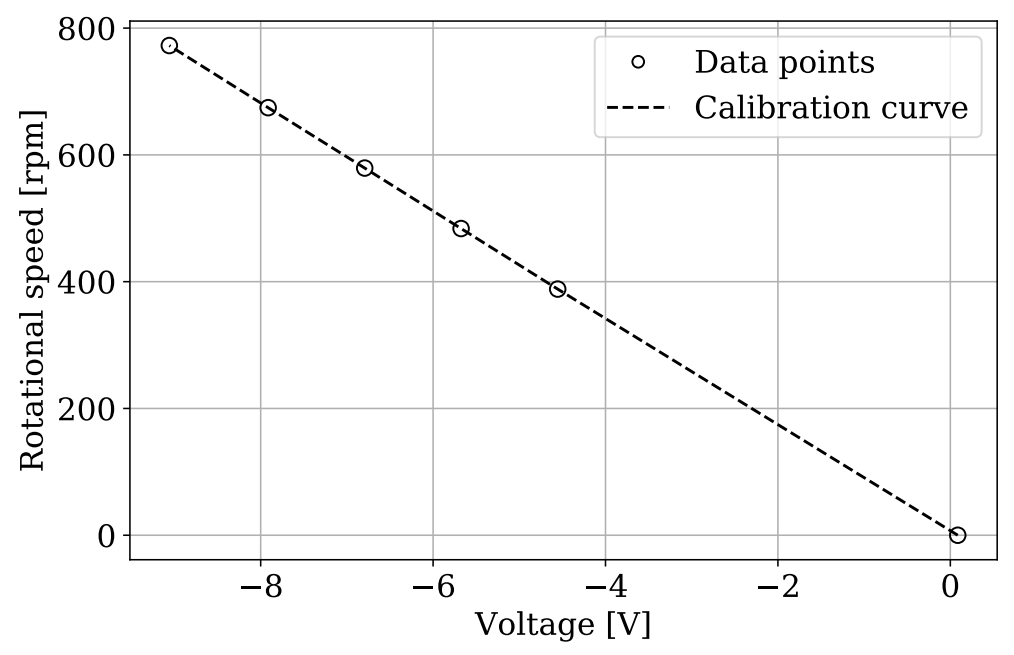

Figure G.6: Proximity sensor calibration results 
As before, the CATMAN program is used to fit a $2^{\text {nd }}$ order polynomial curve to the data which is then used for interpolation during testing. An example of the proximity sensor calibration data is given in Table G.3.

Table G.3: Proximity sensor calibration trend data

\begin{tabular}{lcc}
\hline Converter & Equation & $\mathbf{R}^{2}$ \\
\hline Frequency-to-voltage & $N(V)=0,1578 V^{2}-83,0520 V+7,0916$ & 0.9999 \\
\hline
\end{tabular}




\section{Appendix $\mathrm{H}$}

\section{Fan characteristics sample calculation}

During a single test run, the time-averaged results of the four instruments, namely: inlet bellmouth static pressure $\left(\Delta \bar{p}_{s, \text { bell }}\right)$, plenum chamber static pressure $\left(\Delta \bar{p}_{s, p l e n}\right)$, fan torque $\left(\bar{T}_{m}\right)$, and fan rotational speed $\left(\bar{N}_{m}\right)$ are recorded at various flow rates across the fan's operational envelope. The initial and final zero readings are taken immediately before and after each test run and are indicated as such (e.g $\left.\Delta \bar{p}_{s, b e l l, z e r o, i}\right)$. As previously mentioned, the initial and final no load readings are additionally taken to account for the variable bearing loads experienced during start-up and steady operation of the test facility (denoted $T_{N L, i}$ and $T_{N L, f}$, respectively). The initial and final ambient air temperatures and pressures $\left(T_{a, i / f}, P_{a, i / f}\right.$ ) are also taken immediately before and after each test run. An example of the initial zero and no load test data received for a typical test run of the datum fan (with a $34^{\circ}$ blade angle and $6 \mathrm{~mm}$ tip clearance) is given in Tables H.1- H.5 below.

Table H.1: Averaged initial zero reading measurement data of the datum fan

\begin{tabular}{ccc}
\hline Parameter & Variable & Value \\
\hline Torque & $\bar{T}_{m, \text { zero }, i}$ & $-0.3133 \mathrm{~N} . \mathrm{m}$ \\
Speed & $\bar{N}_{m, \text { zero }, i}$ & $-0.0173 \mathrm{rpm}$ \\
Inlet bellmouth pressure differential & $\Delta \bar{p}_{s, \text { bell,zero, } i}$ & $0.1219 \mathrm{~Pa}$ \\
Plenum chamber pressure differential & $\Delta \bar{p}_{s, \text { plen }, \text { zero }, i}$ & $0.6500 \mathrm{~Pa}$ \\
\hline
\end{tabular}


Table H.2: Averaged initial no load measurement data of the datum fan

\begin{tabular}{ccc}
\hline Parameter & Variable & Value \\
\hline Torque & $\bar{T}_{m, N L, i}$ & $3.2902 \mathrm{~N} \cdot \mathrm{m}$ \\
Speed & $\bar{N}_{m, N L, i}$ & $723.1514 \mathrm{rpm}$ \\
\hline
\end{tabular}

Table H.3: Averaged final zero reading measurement of the datum fan

\begin{tabular}{ccc}
\hline Parameter & Variable & Value \\
\hline Torque & $\bar{T}_{m, z e r o, f}$ & $-0.0616 \mathrm{~N} . \mathrm{m}$ \\
Speed & $\bar{N}_{m, z e r o, f}$ & $-0.0099 \mathrm{rpm}$ \\
Inlet bellmouth pressure differential & $\Delta \bar{p}_{s, b e l l, z e r o, f}$ & $0.0836 \mathrm{~Pa}$ \\
Plenum chamber pressure differential & $\Delta \bar{p}_{s, p l e n, z \text { ero }, f}$ & $0.7675 \mathrm{~Pa}$ \\
\hline
\end{tabular}

Table H.4: Averaged final no load measurement of the datum fan

\begin{tabular}{ccc}
\hline Parameter & Variable & Value \\
\hline Torque & $\bar{T}_{m, N L, f}$ & $2.3290 \mathrm{~N} \cdot \mathrm{m}$ \\
Speed & $\bar{N}_{m, N L, f}$ & $722.7986 \mathrm{rpm}$ \\
\hline
\end{tabular}

Table H.5: Initial and final ambient air measurement data of the datum fan

\begin{tabular}{ccc}
\hline Parameter & Variable & Value \\
\hline Initial temperature & $T_{a, i}$ & $13.5^{\circ} \mathrm{C}$ \\
Initial pressure & $p_{a, i}$ & $1011.3 \mathrm{hPa}$ \\
Final temperature & $T_{a, f}$ & $15.0^{\circ} \mathrm{C}$ \\
Final pressure & $p_{a, f}$ & $1011.4 \mathrm{hPa}$ \\
\hline
\end{tabular}

The data obtained for the same fan at the $8^{\text {th }}$ evaluated volumetric flow rate is given in Table H.6. 
Table H.6: Averaged measurement data of the datum fan evaluated at the $8^{\text {th }}$ volumetric flow rate

\begin{tabular}{ccc}
\hline Parameter & Variable & Value \\
\hline Torque & $\bar{T}_{m}$ & $43.2354 \mathrm{~N} . \mathrm{m}$ \\
Speed & $\bar{N}_{m}$ & $722.2875 \mathrm{rpm}$ \\
Inlet bellmouth pressure differential & $\Delta \bar{p}_{s, \text { bell }}$ & $137.5387 \mathrm{~Pa}$ \\
Plenum chamber pressure differential & $\Delta \bar{p}_{s, \text { plen }}$ & $159.3778 \mathrm{~Pa}$ \\
\hline
\end{tabular}

Using this information, the data obtained during each test run is first corrected for the zero offset by subtracting the mean of the zero reading measurements with

$$
\phi_{\text {corrected }}=\bar{\phi}-\bar{\phi}_{z e r o, i / f}
$$

Due to the significant change in the initial and final no load torque values, the corrected value is calculated by subtracting a linearly interpolated value between the two no load readings. This is represented mathematically by:

$$
T_{m, \text { corrected }}=\bar{T}_{m}-\left(T_{N L, i}-\frac{(i-1)\left(T_{N L, i}-T_{N L, f}\right)}{n-1}\right)
$$

where $i$ represents the current interpolation point (starting from 1) and $n$ represents the total number of evaluated flow rates $(n=10$ for this example). Using these equations along with the values given in Table H.6, the corrected measurement values (represented by dropping the bar superscript) of the current example are given in Table H.7.

Table H.7: Corrected measurement data of the datum fan evaluated at the $8^{\text {th }}$ volumetric flow rate

\begin{tabular}{ccc}
\hline Variable & Correction value & Corrected value \\
\hline$T_{m}$ & $2.5426 \mathrm{~N} . \mathrm{m}$ & $40.6929 \mathrm{N.m}$ \\
$N_{m}$ & $-0.0136 \mathrm{rpm}$ & $722.3011 \mathrm{rpm}$ \\
$\Delta p_{s, \text { bell }}$ & $0.1027 \mathrm{~Pa}$ & $137.4359 \mathrm{~Pa}$ \\
$\Delta p_{s, \text { plen }}$ & $0.7088 \mathrm{~Pa}$ & $158.6690 \mathrm{~Pa}$ \\
\hline
\end{tabular}

The ambient air density is calculated from the universal gas law as 


$$
\begin{aligned}
\rho_{a t m} & =\frac{p_{a t m}}{R T_{a t m}} \\
& =\frac{1011,35 \times 100}{287 \times(14,25+273,15)} \\
& =1,2261 \mathrm{~kg} / \mathrm{m}^{3}
\end{aligned}
$$

where $R$ represents the specific gas constant of air. Average values taken before and after testing (Table H.5) are used to represent the ambient conditions with $T_{a t m}=T_{a, a v g}$ and $p_{a t m}=p_{a, a v g}$. The plenum density is calculated as

$$
\begin{aligned}
\rho_{\text {plen }} & =\frac{p_{a t m}-\Delta p_{s, p l e n}}{R T_{\text {atm }}} \\
& =\frac{1011,35 \times 100-158,6690}{287 \times(14,25+273,15)} \\
& =1,2242 \mathrm{~kg} / \mathrm{m}^{3}
\end{aligned}
$$

The mass flow rate through the test facility is calculated by

$$
\begin{aligned}
\dot{m} & =K_{n} \frac{\pi D_{\text {bell }}^{2}}{4} \sqrt{2 \rho_{\text {atm }} \Delta p_{\text {s,bell }}} \\
& =0,9803 \times \frac{\pi \times 1,008^{2}}{4} \sqrt{2 \times 1,2261 \times 137,4359} \\
& =14,3616 \mathrm{~kg} / \mathrm{s}
\end{aligned}
$$

where $K_{n}=0,9803$ for the test facility's inlet loss coefficient and $D_{\text {bell }}=1.008 \mathrm{~m}$ (Venter and Kroger, 1990). Subsequently, the dynamic pressure component can be calculated using

$$
\begin{aligned}
p_{d, p l e n} & =\frac{\dot{m}^{2}}{2 \rho_{\text {plen }} A_{\text {plen }}^{2}} \\
& =\frac{14,3616^{2}}{2 \times 1,2242 \times 16^{2}} \\
& =0,3291 \mathrm{~Pa}
\end{aligned}
$$

where $A_{\text {plen }}=16 \mathrm{~m}^{2}$ represents the cross-sectional area of the plenum chamber. The total-to-static pressure drop across the fan can then be calculated by 


$$
\begin{aligned}
\Delta p_{t-s}^{\prime} & =-\left(\Delta p_{s, p l e n}+p_{d, p l e n}\right) \\
& =-(-158,6690+0,3291) \\
& =158,3399 \mathrm{~Pa}
\end{aligned}
$$

Fan power is calculated by using the fan rotational speed $N_{m}$ and measured torque $T_{m}$, with

$$
\begin{aligned}
P^{\prime} & =\frac{2 \pi N_{m}}{60} T_{m} \\
& =\frac{2 \times \pi \times 722,3011}{60} \times 40,6929 \\
& =3077,9749 \mathrm{~W}
\end{aligned}
$$

Using Equations H.4 and H.5, the volumetric flow rate is calculated by

$$
\begin{aligned}
\dot{V}^{\prime} & =\frac{\dot{m}}{\rho_{\text {plen }}} \\
& =\frac{14,3616}{1,2242} \\
& =11,7314 \mathrm{~m}^{3} / \mathrm{s}
\end{aligned}
$$

The fan total-to-static efficiency is calculated by

$$
\begin{aligned}
\eta_{t-s}^{\prime} & =\frac{\dot{V}^{\prime} \Delta p_{t-s}^{\prime}}{P^{\prime}} \\
& =\frac{11,7314 \times 158,3399}{3077,9749} \\
& =0,6035
\end{aligned}
$$

Finally, the fan characteristics calculated in Equations H.7- H.10 require scaling in order to be representative of the fan's operating conditions at $\rho_{D}=1.2 \mathrm{~kg} / \mathrm{m}^{3}$ and $N_{D}=722 \mathrm{rpm}$. This is achieved by using the fan scaling laws, with

$$
\begin{aligned}
\dot{V} & =\dot{V}^{\prime}\left(\frac{N_{D}}{N}\right) \\
\Delta p_{t-s} & =\Delta p_{t-s}^{\prime}\left(\frac{N_{D}}{N}\right)^{2}\left(\frac{\rho_{D}}{\rho}\right) \\
P & =P^{\prime}\left(\frac{N_{D}}{N}\right)^{3}\left(\frac{\rho_{D}}{\rho}\right) \\
\eta_{t-s} & =\eta_{t-s}^{\prime}
\end{aligned}
$$


The final corrected fan performance characteristics are given in Table H.8.

Table H.8: Fan performance characteristics of the datum fan evaluated at the $8^{\text {th }}$ volumetric flow rate

\begin{tabular}{cc}
\hline Variable & Value \\
\hline$\dot{V}$ & $11.7265 \mathrm{~m}^{3} / \mathrm{s}$ \\
$\Delta p_{t-s}$ & $155.0810 \mathrm{~Pa}$ \\
$P$ & $3013.3686 \mathrm{~W}$ \\
$\eta_{t-s}$ & 0.6035 \\
\hline
\end{tabular}

